\title{
Computer aided detection of defects in FRP bridge decks using infrared thermography
}

\author{
Gajanan M. Lonkar \\ West Virginia University
}

Follow this and additional works at: https://researchrepository.wvu.edu/etd

\author{
Recommended Citation \\ Lonkar, Gajanan M., "Computer aided detection of defects in FRP bridge decks using infrared \\ thermography" (2005). Graduate Theses, Dissertations, and Problem Reports. 1672. \\ https://researchrepository.wvu.edu/etd/1672
}

This Thesis is protected by copyright and/or related rights. It has been brought to you by the The Research Repository @ WVU with permission from the rights-holder(s). You are free to use this Thesis in any way that is permitted by the copyright and related rights legislation that applies to your use. For other uses you must obtain permission from the rights-holder(s) directly, unless additional rights are indicated by a Creative Commons license in the record and/ or on the work itself. This Thesis has been accepted for inclusion in WVU Graduate Theses, Dissertations, and Problem Reports collection by an authorized administrator of The Research Repository @ WVU. For more information, please contact researchrepository@mail.wvu.edu. 


\title{
Computer Aided Detection of Defects in FRP Bridge Decks Using Infrared Thermography
}

\author{
By \\ Gajanan M. Lonkar \\ THESIS \\ Submitted to the \\ College of Engineering and Mineral Resources \\ West Virginia University
}

In partial fulfillment of the requirements for the degree of

Master of Science in Electrical Engineering

Powsiri Klinkhachorn, Ph.D., Chair

Arun Ross, Ph.D.

Udaya B. Halabe, Ph.D., P.E.

Hota V. S. GangaRao, Ph.D., P.E.

Lane Department of Computer Science and Electrical Engineering

Morgantown, West Virginia

2005

Keywords: FRP, Defects, Debonds, Delaminations, Infrared Thermography, Image Processing, Computer Aided Detection, Mosaicking 


\section{Abstract \\ Computer Aided Detection of Defects in FRP Bridge Decks Using Infrared Thermography}

\section{Gajanan Lonkar}

The objective of this research is to develop a turn-key system that is able to interface with the FLIR ThermaCAM S60 infrared camera and automatically capture and analyze defects in infrared images of FRP bridge decks. Infrared thermography is one of the nondestructive evaluation (NDE) techniques that are being used to locate defects (debonds and delaminations) in bridge components. It is a rapid data collection and interpretation technique having high sensitivity and reliability. Analysis of infrared images by human interpretation is dependent on the users knowledge and hence introduces ambiguity in the defect detection process

This thesis investigates the use of an automated defect detection system to locate defects in infrared images of FRP bridge decks to eliminate/reduce human intervention. Air-filled and water-filled debonds were inserted between the wearing surface and the underlying FRP deck. Also, simulated subsurface delaminations (of various sizes and thickness) were created at the flange-to-flange junction between two FRP deck modules. Infrared thermography was used to capture an image of these FRP bridge decks under various heating and cooling methods, distance variations etc. A turn key system developed as a part of this research was able to interface with the infrared camera, capture infrared images and analyze them for defects automatically. The effect of these conditions on the accuracy of the automated defect detection algorithms was assessed.

Limitations of using a single image to assess the physical location of the defect on the entire bridge deck were observed. Mosaicking of a sequence of infrared images to form a "composite image" mosaic was suggested. The mosaic will be a single image representation of the area covered by all the infrared images combined. Software assisted and hardware assisted methods were explored to develop a real time automated mosaicking and defect detection system.

The results show that automated detection of defects in infrared images of FRP bridge decks was performed successfully. More than 90\% accuracy in defect detection was obtained in the tests conducted under various conditions in the laboratory environment. The system was able to detect the defects accurately at par or sometimes better than that possible by the human eye. Software and hardware assisted mosaicking schemes were tested. The hardware assisted scheme was found to be more suitable for real time implementation. 


\section{Acknowledgements}

Some of my friends say that I am a really lucky guy. When I myself look back in the previous years I cannot agree more. I consider myself fortunate to be surrounded by a heart warming family, caring friends and knowledgeable teachers. They all have enriched my life and guided me through tough times.

I have been privileged to have Dr. Powsiri Klinkhachorn as my advisor. He has guided me not only through various stages of this research but also during my studies at WVU. His attitude of perfection, keen attention to detail and motivation to remain focused, has kept me on my toes. This has helped hone my academic knowledge as well as my writing and presentation skills, which are lifetime benefits. I am also grateful to Dr. Udaya Halabe, Dr. Gangarao Hota and Dr. Arun Ross for their expert guidance in the completion of my research and academic coursework. I would also like to thank the faculty members of the LCSEE Department for their academic guidance and encouragement.

I take this opportunity to thank my parents, sisters and brother for supporting and guiding me in my decisions. I am lucky to be gifted with good friends both in the United Stated and India. I want to especially mention Amit, Andy, Vivek, Modi, Shantanu Sanket and Swetha for adding humour in the most serious situations and making everyday life an enjoyable experience. Also worth mentioning are Archana, Roy and other students from the NDT lab and structural engineering lab who have directly or indirectly helped in my research work.

I would like to thank the Constructed Facilities Center at West Virginia University for funding this research as a part of a research grant from U. S. Department of Transportation - Federal Highway Administration (USDOT-FHWA). 


\section{Dedication}

I would like to dedicate this thesis to my parents Madhav Lonkar and Manik Lonkar for their sacrifices, planning of our futures and continuous prayers for our welfare. 


\section{Contents}

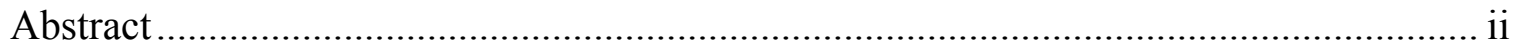

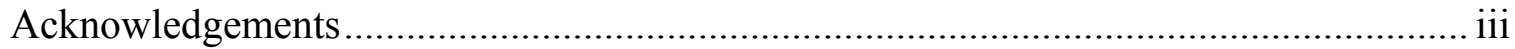

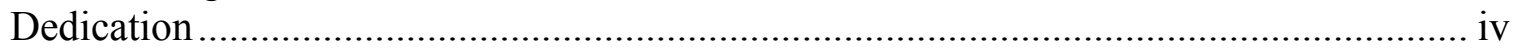

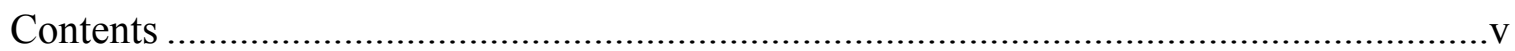

List of Figures and Illustrations ................................................................................ viii

List of Symbols, Abbreviations and Nomenclature ...................................................... xii

CHAPTER 1. INTRODUCTION ...............................................................................1

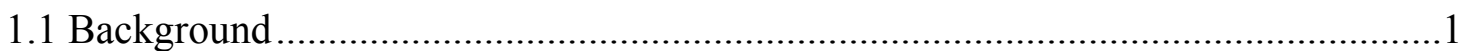

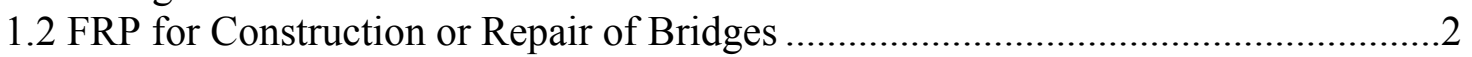

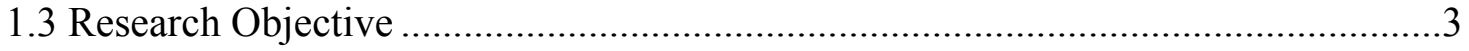

1.4 Scope

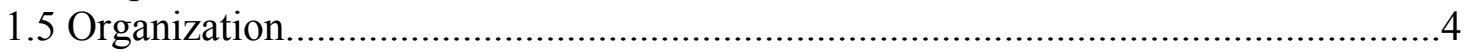

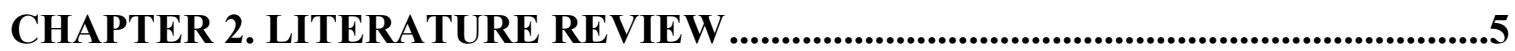

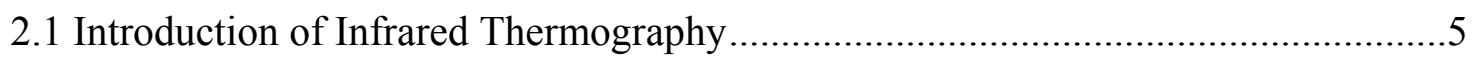

2.2 NDE Techniques for Defect Detection in Bridges ..................................................

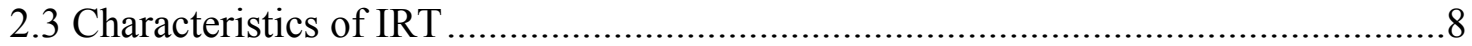

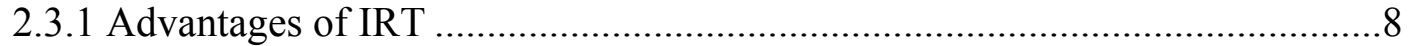

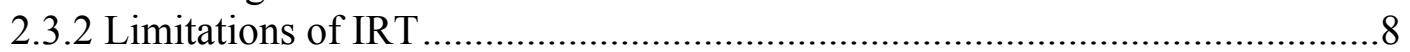

2.4 Measuring Temperature by Infrared Thermography .............................................9

2.4.1 The Electromagnetic Spectrum ....................................................................

2.4.2 Infrared Radiation in the Electromagnetic Spectrum ...................................10

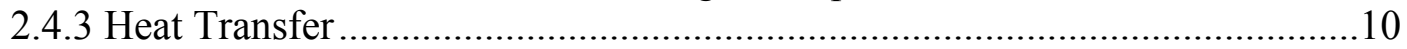

2.4.4 Conversion of Infrared Radiation to Temperature ...........................................11

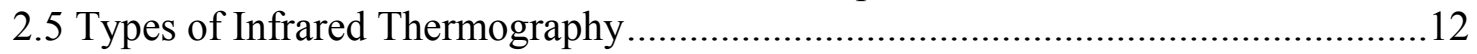

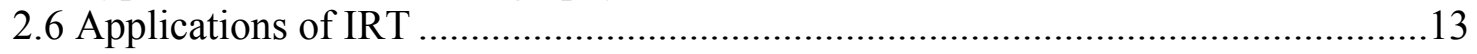

2.6.1 Plant Condition Monitoring and Maintenance …………................................14

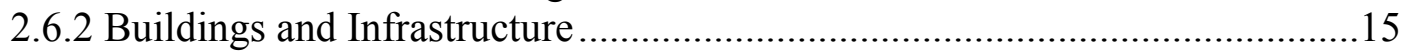

2.6.3 Production Testing and Monitoring …………...........................................16

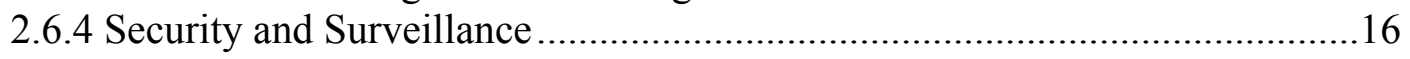

2.7 Infrared Thermography for Defect Detection .........................................................

2.8 Review on Automated Defect Detection .........................................................29

2.8.1 Automated defect detection approaches....................................................29

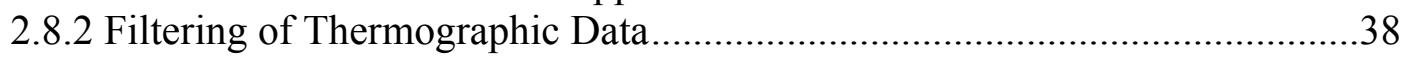

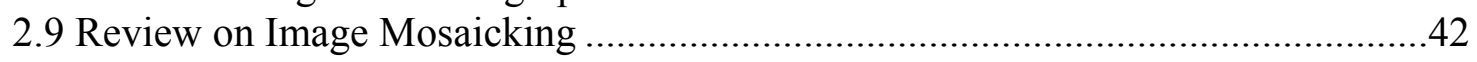

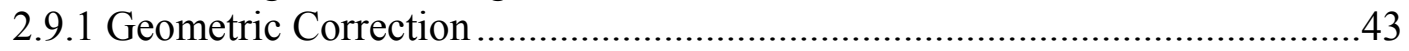

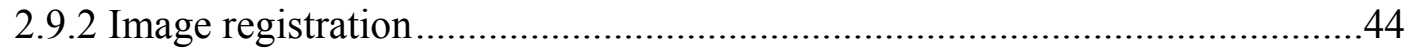

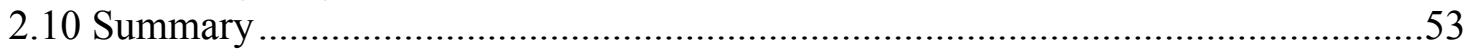


CHAPTER 3. AUTOMATED DEFECT DETECTION SYSTEM..............................54

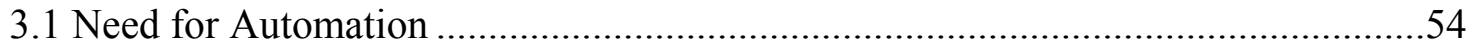

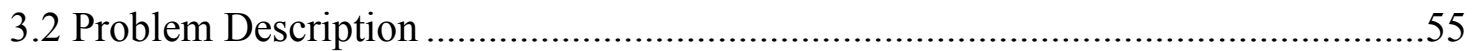

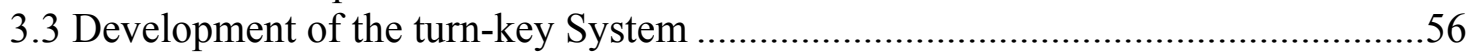

3.3.1 AutoIT Scripting Language Method .........................................................57

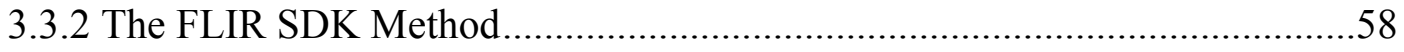

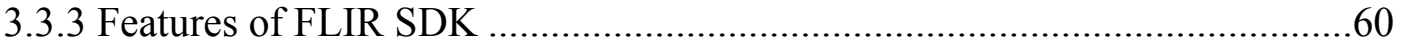

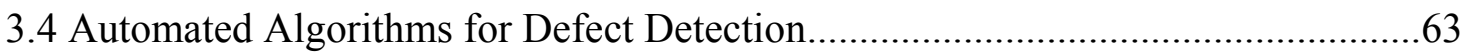

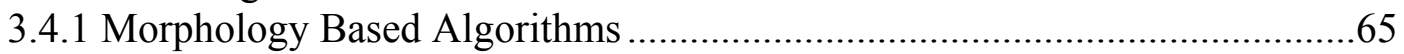

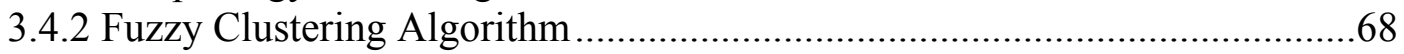

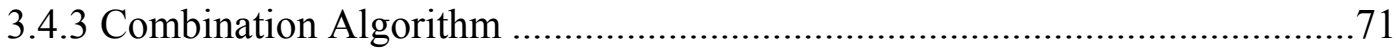

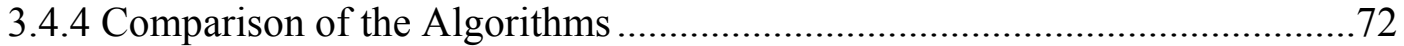

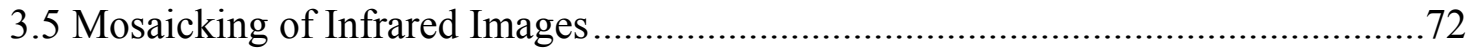

3.5.1 Hardware Assisted Mosaicking ....................................................................

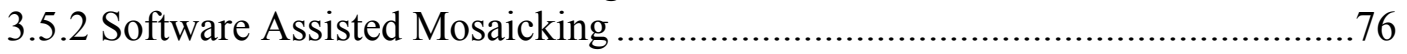

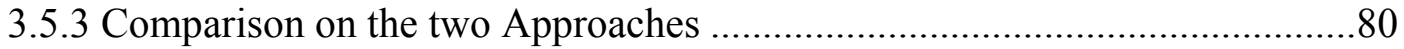

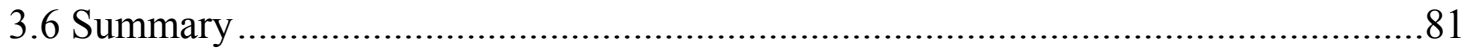

CHAPTER 4. EXPERIMENTAL SETUP AND RESULTS .......................................82

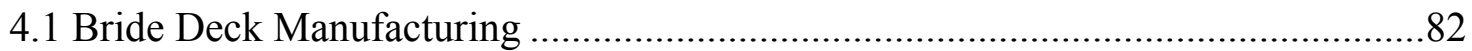

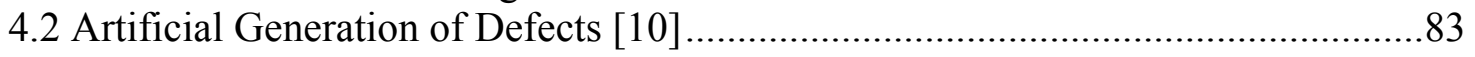

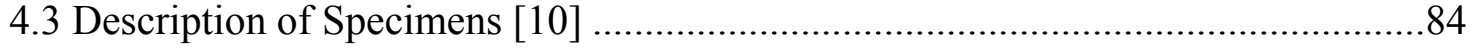

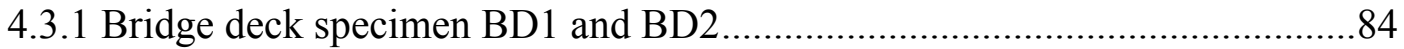

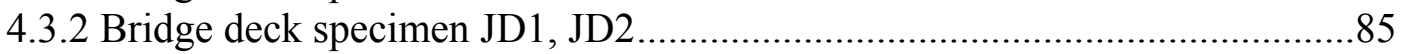

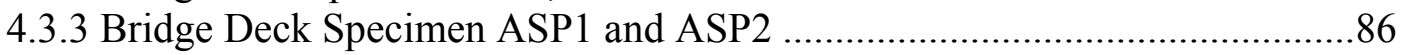

4.3.4 Bridge Deck Specimens WJD1 WJD2 and WJD3 ……................................86

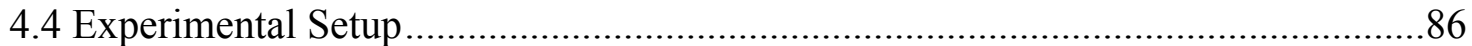

4.4.1 FLIR ThermaCAM S60 Infrared Camera …………....................................8

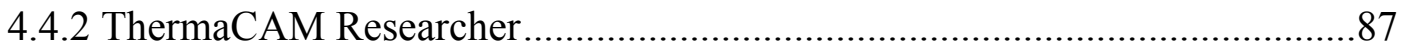

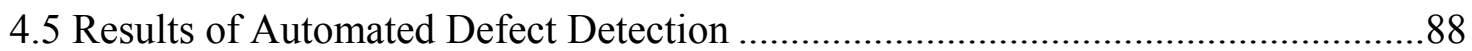

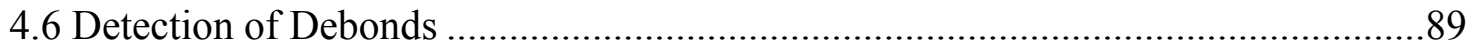

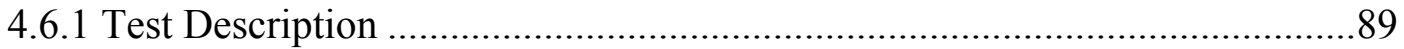

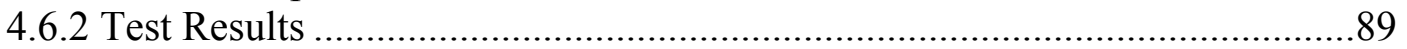

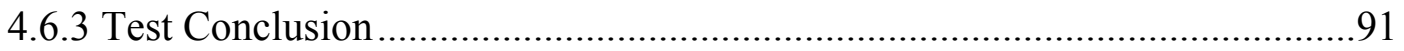

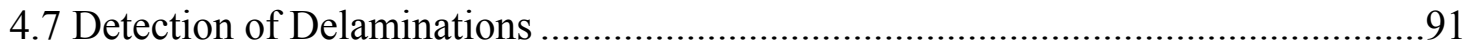

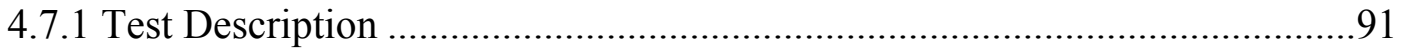

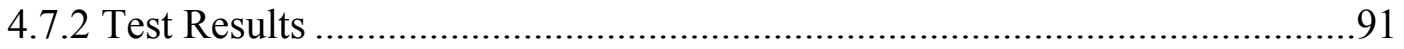

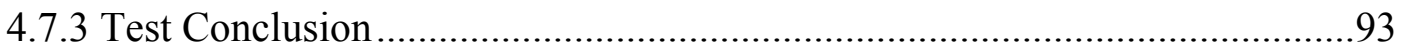

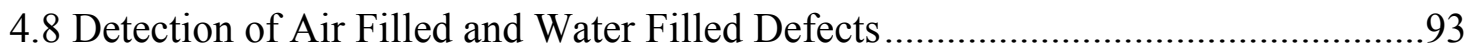

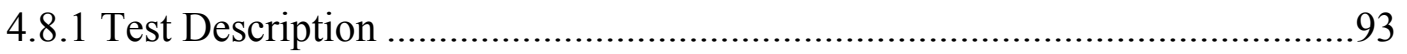

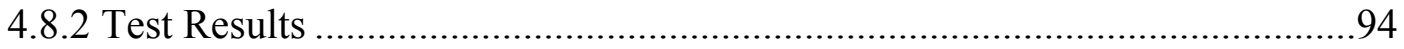

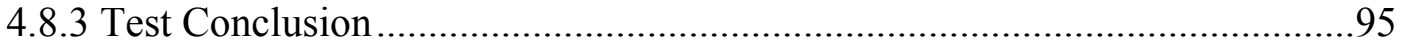

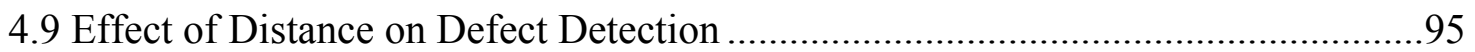

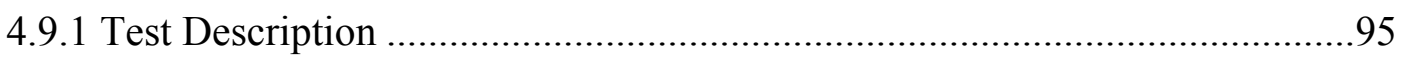

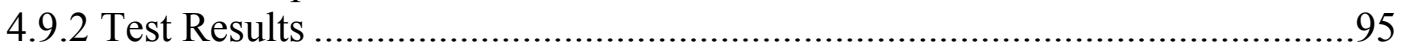

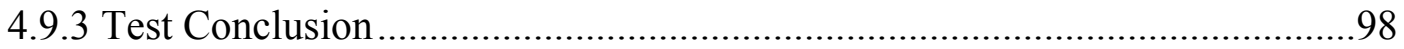

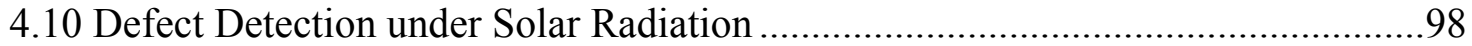




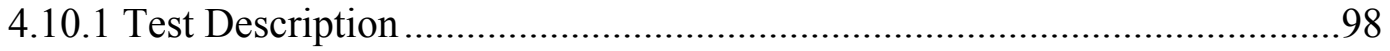

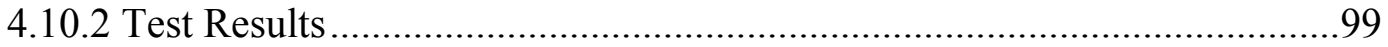

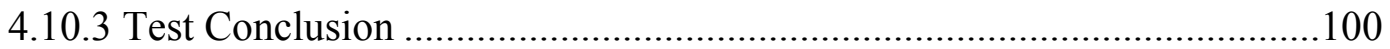

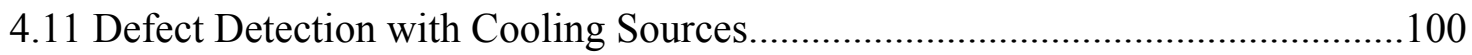

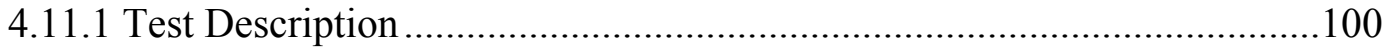

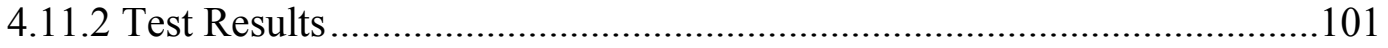

4.11.3 Test Conclusion ................................................................................102

4.12 Defect Detection on Infrared Images of Field Tests ............................................103

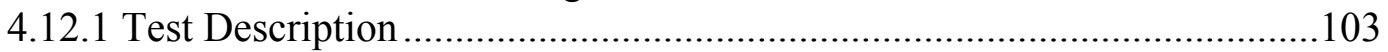

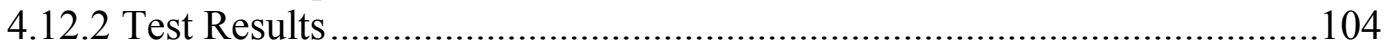

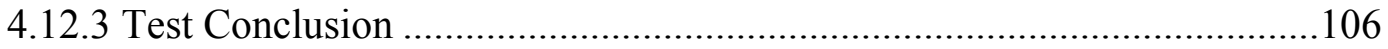

4.13 Performance of Defect Detection Algorithms ……..........................................107

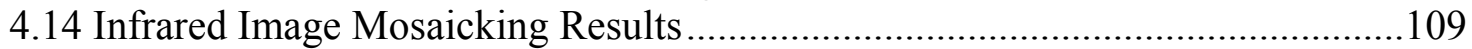

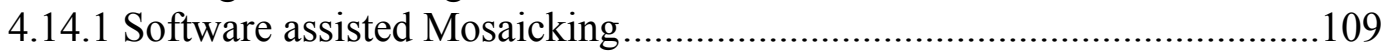

4.14.2 Performance of Software assisted mosaicking .......................................111

4.14.3 Hardware assisted mosaicking .......................................................112

4.14.4 Performance of Hardware assisted Mosaicking........................................113

4.15 Conclusions for Infrared Image Mosaicking ....................................................114

CHAPTER 5. CONCLUSIONS AND FUTURE WORK ..........................................116

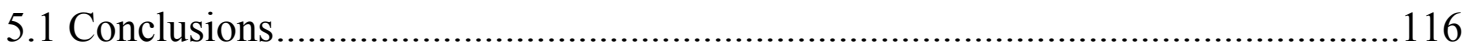

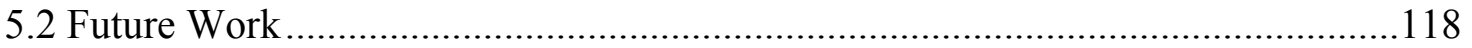

REFERENCES................................................................................................................120

APPENDIX A 


\section{List of Figures and Illustrations}

Figure 2.1: The electromagnetic spectrum [11]. 1: X-ray; 2: UV; 3: Visible; 4: IR; 5: Microwaves; 6: Radiowaves.

Figure 2.2: General setup of infrared thermography used in NDE [14] ......................... 12

Figure 2.3: Infrared image for excessive heating of a connecting clip [13] .................... 14

Figure 2.4: Thermogram of a roof with moisture saturation [13]................................... 15

Figure 2.5: The cross section of the composite bridge deck specimen $[10,18] \ldots \ldots \ldots \ldots \ldots . . .17$

Figure 2.6: (a) Photograph, and (b) Infrared image of Sample 1 [18] ........................... 18

Figure 2.7: (a) Schematic of the FRP deck Sample (b) Infrared image of the Sample [18].

Figure 2.8: Infrared images showing (a) hotter areas caused due to reflection of solar rays by the guard rails, and (b) colder areas caused due to shadows from guard rails [18].

Figure 2.9: (a) Tube \#3 after 4 minutes of cooling. Time dependent boundary layer thermal pattern begins to appear. (b) - Tube \#3 after 8 minutes of cooling. Fully developed thermal pattern shown [19]....

Figure 2.10: (a)Tube \# 4 after 4 minutes of heating and 4 minutes of cooling.

(b)Tube \#2 with water in debond and no wear surface [19].

Figure 2.11: (a) Schematic view of the infrared thermography set up in the laboratory

(b) Experimental setup [12].

Figure 2.12: Box section specimens (a) Box1: 2"x2" delamination with 1/16"

thickness (b)Box3: 3 "x3" delamination with 1/16" thickness (c)Box4: one 3"x3" and another 2"x2" delamination with 1/16" thickness [12].

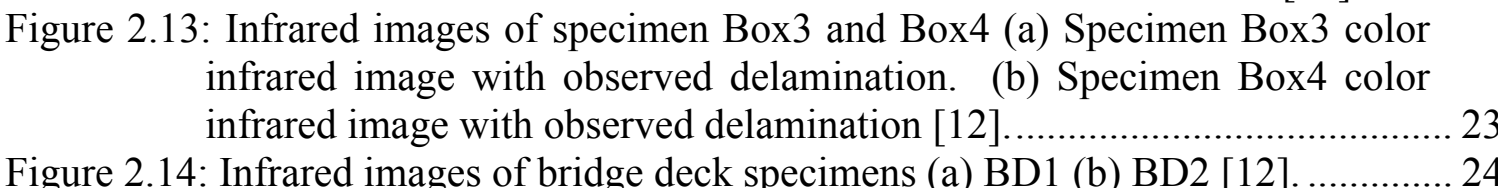

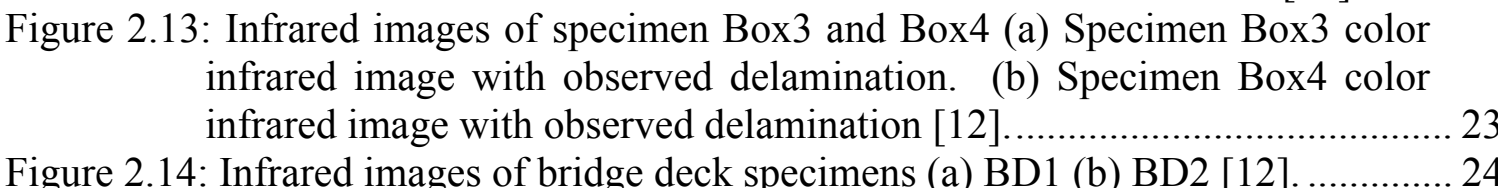

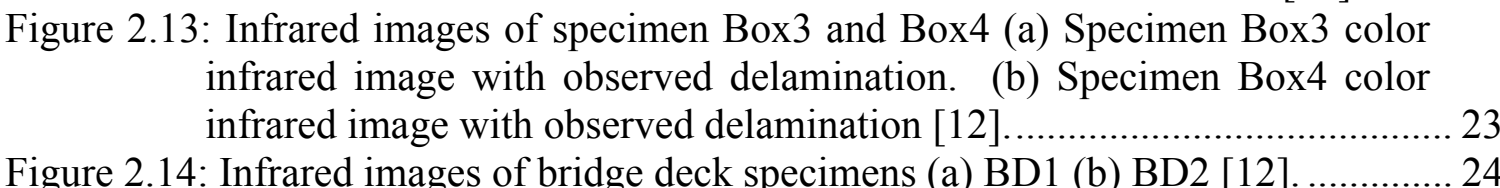

Figure 2.15: (a) Front view and cross-sectional view of the GFRP bridge deck specimen BD1 with w/s (b) Schematic view of location of debonds in bridge deck specimens (a) BD1a (b) BD1b [10].

Figure 2.16: Infrared images of Bridge deck BD1 and BD2 (a) Infrared image of BD1a with debonds of sizes 2"x2" and 3"x3" (b) Infrared image of deck BD2 (c) JD1a with 3"x3" delamination (b) JD1b with the 1"x1" and 2"x2" delaminations [10].

Figure 2.17: Infrared image of bridge deck specimen JD1a with delamination of size 3"x3" at (a) 9.30 A.M. (b) 12.30 P.M. (c) 2.30 P.M. (d) 4.30 P.M [10] ..... 27

Figure 2.18: (a) Infrared image of the 2"x2" and 4"x4" debonds in specimen ASP1 with 2" wearing surface (b) Infrared image of 3"x3" air-filled debond in ASP2 heated with the shop heater in horizontal position [4].

Figure 2.19: (a) Infrared image of specimen WJD1 side 2 without any wearing surface (b) Close up of the $3 \times 3$ "x1/16" water-filled delamination in WJD2 [4]. 
Figure 2.20: (a)(b) Infrared image of aluminium honeycomb with bonding defect and the processing result $(c)(d)$ Infrared image of graphite epoxy panel with artificial defect and processing result [23].

Figure 2.21: Images of a composite panel with 5 square $(20 \times 20 \mathrm{~mm})$ flat bottom cavities. (a) background image (b) thermal image at $7 \mathrm{~s}$ after the flash (c) thermal response averaged in the interval 10-50 s (d) thermal response averaged in the interval 10-50 $\mathrm{s}$ with the subtracted background; (e) phase image [24].

Figure 2.22: Results of image processing of the (a) Thermograms from Figure 2.21(c) processing result $\quad$ (b) Thermograms from Figure 2.21(c) processing result (c) Phasegram from Figure 2.21 (e) processing result [24]

Figure 2.23: (a) Maximum contrast image for part containing deep sprue defect (b) Maximum slope image for part containing deep sprue defect (c) Image of

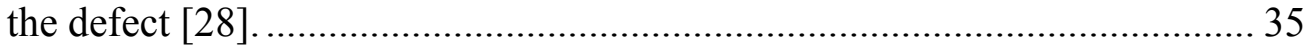

Figure 2.24: On-line inspection system implemented in [30].................................... 36

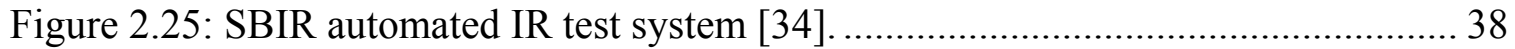

Figure 2.26: (a) A subtle diagonal noise pattern was present in the image. (b) The pattern was changing in time and dominates the slope image. (c) Image with the desired subsurface details for the inspector's evaluation after spatial filtering [25].

Figure 2.27: (a) Raw image of resolution target clusters $G$ and $H$ (Left). (b) Improved image using the Thermal Point Spread Function obtained from TMTF analysis

Figure 2.28: (a) Logarithmic temperature time plots for a background point. (b) raw image data (c) enhanced image using TSR approach. [27]

Figure 2.29:(a) LWIR image registered with visible image (b) SWIR image registered with visible image [37].

Figure 2.30: (a) The original IR image. (b) The original even polarized SAR image.

(c) The original odd polarized SAR image. (d) The smoothed SAR image registered with the IR image. (e) The fused image [38].

Figure 2.31: A sequence of images mosaicked together utilizing all available cross linked image correspondences [39].

Figure 2.32: (a) A two image mosaic with seam (b) Removal of the seam after blending [39]....

Figure 2.33: Video acquisition setup using a camera mounted on a mobile platform [41]

Figure 2.34: (a) Mosaicking without and with blending for the visual sequence. (b)

Mosaicking without and with blending for the thermal sequence [41] ........ 52

Figure 2.35: (a) Sample frames from infrared sequence. (b) mosaic generated from the infrared sequence [41]. ................................................................ 53

Figure 3.1: FLIR SDK demo screenshot with explanation of the GUI ......................... 59

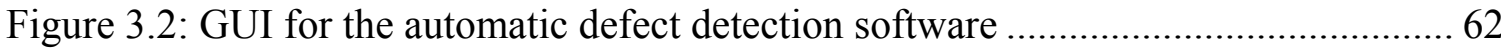

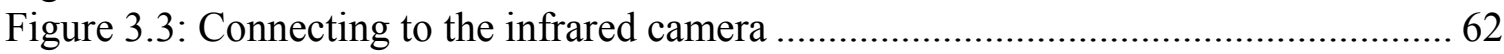

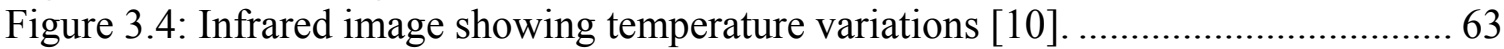

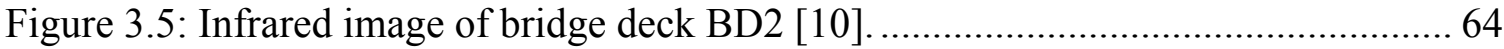


Figure 3.6: (a) Infrared image of bridge deck BD1a with $3 \times 3$ " and $2 \times 2$ " defects [10].

(b) $3 \mathrm{D}$ view of the infrared image shown in Figure 3.6(a).

Figure 3.7: Various stages of morphological defect detection algorithm......................... 68

Figure 3.8: Cluster map of the image shown in Figure 3.6(a)........................................ 70

Figure 3.9: Various stages of fuzzy clustering based defect detection algorithm. ........... 71

Figure 3.10: Block diagram of the combination algorithm........................................... 72

Figure 3.11: Setup for infrared image mosaicking. ……….......................................... 74

Figure 3.12: (a) Algorithm for hardware assisted image mosaicking. (b) Example of

a hardware assisted mosaic with the detected defects. ................................... 75

Figure 3.13: (a)(b) Infrared images to be mosaicked along with the set of control

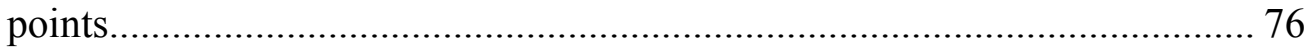

Figure 3.14: (a)(b) Matching pair of control points among the images shown in

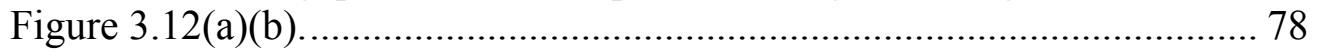

Figure 3.15: Algorithm for software assisted Image mosaicking................................... 79

Figure 3.16: Example result of the software assisted mosaicking algorithm. ................. 80

Figure 4.1: Photographs showing manufacturing of an FRP bridge deck in a factory using the pultrusion process at Bedford Reinforced Plastics [10]................. 83

Figure 4.2: Close up view of the 3"x3" delamination, created at the flange-flange junction of a module (a) without wax paper (b) with wax paper [10]........... 84

Figure 4.3: (a) Front view and cross-sectional view of the GFRP bridge deck

$\begin{array}{ll}\text { specimen BD2 } & \text { (b) schematic view of location of debonds in BD2 }\end{array}$ [10].....

Figure 4.4: Photograph of FLIR ThermaCAM ${ }^{\mathrm{TM}} \mathrm{S} 60$ infrared camera.......................... 87

Figure 4.5: Window of a ThermaCAM Researcher 2002 showing different features [4] 88

Figure 4.6: (a) Infrared image of bridge deck BD1 side A with 3"x3" and 2"x2" debonds. (b) Infrared image of bridge deck BD1 side B with 1"x1" and 0.5 " $\mathrm{x} 0.5$ " debonds.

Figure 4.7: (a) Infrared image of bridge deck BD1 with 3"x3" and 2"x2" debonds under different heating condition (b) Infrared image of bridge deck BD1 with 1 " $\mathrm{x} 1$ " and 0.5 " $\mathrm{x} 0.5$ " debonds under different heating conditions........ 91

Figure 4.8: (a) Infrared image of bridge deck JD2 having 3"x3" delamination with wearing surface (b) Infrared image of bridge deck JD1 having 1"x1" and $2 " \mathrm{x} 2$ " delamination without wearing surface.

Figure 4.9: Front view and cross-sectional view of the FRP bridge deck JD1 w/o wearing surface [10].

Figure 4.10: (a) Infrared image of specimen JD2 with 3"x3" air filled delamination. (b) Infrared image of specimen BD1 with 3"x3" and 2"x2" air filled debond. (c) Infrared image of specimen BD2 with 3"x3" and 2"x2" water filled and 3"x3" air filled debonds. Algorithm set to detect water filled debonds. (d) BD2 with algorithm set to detect water and air filled debonds.

Figure 4.11: Infrared images of specimen BD1a with debonds of sizes 2"x2" and

3"x3" detected at a distance of (a) 3m (b) 4m......................................... 96

Figure 4.12: Infrared images of specimen BD1a with debonds of sizes 2"x2" and 3 "x3" detected at a distance of (a) $5 \mathrm{~m}$ (b) $6 \mathrm{~m}$. 
Figure 4.13: (a) Infrared image of specimen JD1 having 1"x1" and 2"x2" delaminations at a distance of 3 meters with the detected defects. (b) Infrared image of specimen JD1 having 1"x1" and 2"x2" delaminations at a distance of 4 meters with the detected defects. (c) Infrared image of specimen JD1 having 1"x1" and 2"x2" delaminations at a distance of 5 meters with the detected defects.

Figure 4.14: (a) Infrared image of specimen BD1 with 3"x3" debonds at 9am, with the detected defects. (b) Infrared image of specimen BD1 with 3"x3" debonds at $2 \mathrm{pm}$, with the detected defects. (c) Infrared image of specimen BD1 with 3"x3" debonds at 4pm, with the detected defects.

Figure 4.15:(a) Infrared image of specimen JD1 with 3"x3" delamination at 9:30am, with the detected defects. (b) Infrared image of specimen JD1 with 3"x3" delamination at 2:30pm, with the detected defects. (c) Infrared image of specimen JD1 with 3"x3" delamination at 4:30pm, with the detected defects.

Figure 4.16: (a) Infrared image of specimen BD1a with debonds of size 2" $\mathrm{x} 2$ " and 3"x3", cooled using liquid $\mathrm{CO}_{2}$ with defect detection results. (b) Infrared image of (a) with an inverted color scale for the temperature with defect detection results.

Figure 4.17: (a) Infrared image of specimen JD1b with 1"x1" and 2"x2" delaminations, kept in solar radiation and cooled with water. (b) Infrared image of specimen BD1a with 3"x3" debonds, kept in solar radiation and cooled with water

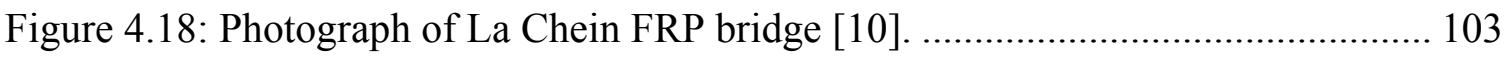

Figure 4.19: Schematic view of the grids [10]......................................................... 104

Figure 4.20: (a) Photograph of grid 15 (b) Infrared image with detected defects of debonds in grid 15.

Figure 4.21: (a)(b): Infrared image of a two debonds in grid 4 respectively................. 105

Figure 4.22: (a) Photograph of the guard rail area (b) Infrared image with the detected defects showing the shadow of the guard rail.

Figure 4.23: (a) Infrared image with dirt on surface (b) Infrared image showing dirt and loose gravel.

Figure 4.24: (a) Defect detection accuracy with laboratory heating and cooling. (b) Defect detection accuracy with laboratory heating and cooling and solar radiation.

Figure 4.25 (a)(b)(c): Infrared images of the FRP bridge deck having movement only in the horizontal and vertical direction.

Figure 4.26: Result of mosaicking infrared images from Figure 4.25(b)(c) ................. 110

Figure 4.27: Result of mosaicking infrared images from Figure 4.25(a)(b)................. 110

Figure 4.28: (a) Example of mosaicking 3 infrared images from a sequence. (b) Example of mosaicking 7 infrared images from a sequence.

Figure 4.29: Result of hardware assisted mosaicking.............................................. 113

Figure 4.30: Setup for the hardware assisted mosaicking. Each polygon within the white dotted lines denotes the capture area of a single infrared image. ..... 114

Figure 4.31: Result of hardware assisted mosaicking........................................... 115 


\section{List of Symbols, Abbreviations and Nomenclature}

$\begin{array}{ll}\text { Symbol } & \text { Definition } \\ \text { IR } & \text { Infrared } \\ \text { IRT } & \text { Infrared Thermography } \\ \text { FRP } & \text { Fiber Reinforced Polymer } \\ \text { GFRP } & \text { Glass Fiber Reinforced Polymer } \\ \text { NDE } & \text { NonDestructive Evaluation } \\ \text { SAR } & \text { Synthetic Aperture Radar } \\ \text { AE } & \text { Acoustics Emission } \\ \text { GPR } & \text { Ground Penetrating Radar } \\ \text { PT } & \text { Pulse Thermography } \\ \text { LT } & \text { Lockin Thermography } \\ \text { VT } & \text { Vibro Thermography } \\ \text { DFT } & \text { Discrete Fourier Transform } \\ \text { NI } & \text { National Instruments } \\ \text { MTF } & \text { Modulation Transfer Function } \\ \text { TMTF } & \text { Thermal Modulation Transfer Function } \\ \text { WMMR } & \text { Weighted Mean of Minimum Range } \\ \text { SWIR } & \text { Short Wave Infrared } \\ \text { LWIR } & \text { Long Wave Infrared } \\ \text { CLAHS } & \text { Contrast Limited Adaptive Histogram Specification } \\ \text { SDK } & \text { Software Development Kit } \\ \text { FCM } & \text { Fuzzy C-Means } \\ \end{array}$




\section{Chapter 1}

\section{Introduction}

\subsection{Background}

The deteriorating state of roadway infrastructure within the United States is one of the most critical transportation issues facing the country. Federal Highway Administration (FHWA) reports that about 27\% of the nation's 594,220 bridges are either structurally deficient or functionally obsolete [1]. Numerous studies further project that repair or replacement of these structures, using only current design practices will be unable to overcome this continuing deterioration at a sufficient rate. With projections into billions of dollars new technologies will be needed to build, evaluate and repair bridges such that their useful life will extend through the next century and permit a progressive improvement of the overall infrastructure. This requirement has been fulfilled by the introduction of new materials for civil infrastructure construction and repair.

The final decade of the twentieth century brought about many technological innovations, especially in regard to high performance materials. These new materials will certainly play a key role in the design and construction of stronger and more durable civil infrastructure systems. Despite the obvious beneficial effects of the emerging construction materials, designers of infrastructure in the twenty first century face new 


\section{CHAPTER 1. INTRODUCTION}

challenges. Barriers that impede implementation include uncertainty about material characteristics, lack of performance data, and inexistence of pertinent design methodologies etc [2].

\subsection{FRP for Construction or Repair of Bridges}

Fiber Reinforced Polymer (FRP) is one such material that is gaining prominence for use in extensive repair and construction of civil and military engineering infrastructure systems. FRP composites are made of fiber reinforcements, resin, fillers, and additives. The fibers provide increased stiffness and tensile capacity. The resin offers high compressive strength and binds the fibers into a firm matrix. The fillers serve to reduce cost and shrinkage. The additives help to improve not only the mechanical and physical properties of the composites but also workability. Detailed information about manufacturing and characteristics of FRP can be found in [3]. FRP bridge decks are being considered for replacing old concrete bridge decks because of their higher strengthto-weight ratio, better durability and low rate of deterioration under severe environmental conditions. FHWA and several states Department of Transportation (DOT) are focusing on advancing the (FRP) composite technology to rebuild the American transportation infrastructure. Many DOTs (Ohio, Missouri, New York, West Virginia, etc.) have funded research programs aimed at full-scale FRP retrofit to strengthen existing structures and validate the design and analysis procedure for FRP based structural systems [4].

The primary barrier to the extensive use of FRP composites in structures is that effective condition monitoring methodologies for FRP need to be developed in order to detect the presence of cracks, onset of failure, estimate the extent of degradation and locate the damaged zone in structural members. Such techniques are necessary to evaluate construction quality and structural integrity after exposure to environmental conditions and harsh loading. FRP bridge decks are susceptible to subsurface debonds and delaminations, which can adversely affect the load carrying capacity and hamper structural integrity. Hence it is necessary to use techniques that will periodically monitor the bridge deck for defect areas and locate the defects in the earliest possible mode without causing any damage to the structure itself. 


\subsection{Research Objective}

This research aims at building a turn key system to automatically detect subsurface defects in infrared images of Glass FRP (GFRP) bridge decks. To accomplish this objective following steps have been undertaken in search of a reliable NDE approach:

$>$ Conduct a literature review on the advances and use of infrared thermography in computer aided detection of defects in FRP bridge decks and other such applications.

$>$ Develop algorithms that can accurately and automatically detect defects in infrared images of FRP bridge decks.

$>$ Test the automated algorithms for accurate detection of defects on infrared images of FRP bridge decks acquired under different environmental and artificially simulated conditions. These conditions include exposure to solar radiation, heating by quartz tower heater, shop heater and also by application of cooling sources like liquid carbon dioxide and cold water etc.

$>$ To test infrared image mosaicking approaches that will enable real time mosaicking of infrared images and generate a composite image map that is used to locate the defects on the FRP bridge deck.

$>$ To develop a turn key system that is able to interface with the camera, acquire and analyze images and generate a mosaic from a sequence of images in real time.

$>$ Provide recommendations for future research to improve the detection accuracy of the computer aided detection algorithms for a more robust detection of subsurface defects in FRP bridge decks.

\subsection{Scope}

The aim of this research was the development of software for automatic detection of defects in Infrared images of FRP bridge decks. The fabrication of FRP bridge decks and artificial introduction of defects (debonds and delaminations) inside the FRP decks was beyond the scope of this research. The decks were manufactured by a pultrusion process at Bedford Reinforced Plastics, Inc., PA. The work of cutting the decks into 


\section{CHAPTER 1. INTRODUCTION}

various sizes, artificially introducing subsurface defects and conducting infrared thermography under various conditions was performed by researches in [4, 10]. A sequence of infrared images of FRP decks under conditions such as exposure to solar radiation, heating by quartz heater, cooling using sources like liquid carbon dioxide was completed in the research by $[4,10]$. The researchers in $[4,10]$ used manual estimation to locate the defects in infrared images of FRP bridge decks. An automated system to affirm their results was developed as a part of this research. The automated algorithms developed as a part of this research, may be used to detect anomalies in infrared images of other modalities such as infrared images of concrete bridge decks, wood etc. The accuracy of detection under other modalities is out of the scope of this research and hence has not been tested. The scope also includes building and testing an infrared image mosaicking scheme that will enable real time mosaicking of infrared images of FRP bridge decks. The final aim is to build a turn-key system that is able to interface with the infrared camera, capture and analyze images and build a composite image map of the bridge deck under consideration.

\subsection{Organization}

This thesis is organized into five chapters. The first chapter gives a brief introduction to the objective and scope of this research. The second chapter explains the fundamentals of infrared thermography. A literature survey on the various applications of infrared thermography, automated defect detection algorithms and image registration and mosaicking approaches for infrared imaging is also discussed in this chapter. The approach taken for automated detection of debonds and delaminations (defects) in FRP bridge decks as well as infrared image mosaicking schemes implemented are explained in the third chapter. The experimental setup for automated defect detection and the results obtained using the developed algorithms are explained in the fourth chapter. Finally, the fifth chapter enlists the conclusions drawn form the research and suggestions for future work. An appendix providing explanation of the working of the GUI built in Matlab, for the development of a prototype turn-key infrared image acquisition and analysis system. 


\section{Chapter 2}

\section{Literature Review}

\subsection{Introduction of Infrared Thermography}

Less than 200 years ago the existence of the infrared portion of the electromagnetic spectrum was not known. It was invented by Sir John Herschel in 1840 . The use of infrared thermography (IRT) to obtain a "heat picture" or a "thermal profile" has advanced from the first time it was used in 1840 by Sir John Herschel [11] to the current technology. The significance and applications of the infrared spectrum, or simply 'the infrared' as a form of heat radiation has increased over the last few decades, especially after the advent of the digital revolution.

The first section of this chapter explains the basic principles of infrared thermography, its applications and explores some of the previous research in various applications of infrared thermography. Next, some of the previous work carried out in the field of infrared thermography, mostly in the field of repair and construction of civil engineering structures is reviewed. This is followed by a literature survey on the use of automated methods for detection of anomalies in infrared images. Finally, a survey of research on image mosaicking in the infrared domain is carried out to explore the use of building a composite image of the entire bridge deck. 


\subsection{NDE Techniques for Defect Detection in Bridges}

Bridges present special inspection problems because they are particularly vulnerable to weather. Varying changes in environmental conditions cause degradation of structural integrity due to formation of cracks below the surface of the bridge. Traditional approaches to the inspection of bridges have included the use of snoopers, man lifts, cranes and scaffolds [5]. These conventional evaluation techniques for bridges rely on physical inspection of structures as a means of determining their condition. The time consuming nature of these techniques significantly increases costs and prohibits regular bridge assessment. They are being increasingly replaced by advanced Non-destructive testing and evaluation (NDE) methods for reliable and robust assessment of aging infrastructure [1]. NDE techniques are used for material evaluation and defect detection in various structural components without any loss of structural integrity. Some of the prominent NDE techniques used for location of defects in civil infrastructures are [6,7]:

1) Acoustic Emission (AE): Cracking or failure of a material is accompanied by a noise (acoustic emissions) that is either audible or inaudible. Acoustic Emissions (AE) are caused by release of strain energy from a material in response to applied stress. This energy is detected using equipment such as piezoelectric transducers, microphones etc. AE sensors require surface contact and can be placed at critical location in the joints to detect crack initiation and growth. In case of large coverage area metal waveguides can be used to propagate waves into the structure. For FRP and steel reinforced concrete, the reinforcement can be used as a waveguide. This method is particularly good for crack monitoring and growth in components made of steel, concrete and timber.

2) Ultrasonics: The ultrasonic technique uses high frequency waves that are generally greater than the $20 \mathrm{kHz}$ and have the potential of detecting surface and subsurface defects in a given member. Ultrasonic energy in the range of $100 \mathrm{kHz}$ to $40 \mathrm{MHz}$ is usually transmitted in the form of pulses through wood concrete or other composite materials. The signal received at the other end is analyzed with respect to wave velocity, attenuation etc to detect defects. Ultrasonic method allows detection of 
deeper and extremely small flaws with high sensitivity and accuracy. The limitation is that the sensors need to be in contact with the surface of the component under inspection.

3) Ground Penetrating Radar (GPR): GPR employs electromagnetic waves to assess dielectric properties of a material and to predict the subsurface condition of the structure, e.g. porosity, delaminations etc. A GPR system is mounted on a cart or moving vehicle. The system has antennas for transmitting waves in the electromagnetic range. It also has receiving and decoding equipment for data analysis. GPR is a non-contact and rapid data collection and defect detection method.

4) Impact Echo: This method is a sonic test for evaluation of integrity and thickness of concrete, timber etc. It involves generation of transient stress pulses in the specimen by mechanical impact on its surface. The pulses generated have a low frequency and hence can penetrate deeper into the surface. A receiving transducer placed adjacent to the point of impact analyzes the reflected echo to detect defects. This technique is suitable for detecting larger anomalies unlike the ultrasound technique.

5) Infrared Thermography (IRT): IRT senses the emissions of thermal radiation from the specimen by using an infrared camera. A thermal image (temperature profile) of the surface is generated from the received thermal signals. The basic concept is that different rates of heat transfer through defect free and defect area lead to surface temperature differentials that are sensed using an infrared camera. Advancements in infrared camera manufacturing technology have made accurate measurements of temperature possible. Temperature difference between two pixels can be measured with an accuracy of $0.05^{\circ} \mathrm{C}$ or less. IRT is a non-contact technique that allows rapid data collection by movement of the infrared camera. It is also a fast data collection and analysis technique. Detailed information about IRT for Non-Destructive Evaluation can be found in $[8,9]$. Another advantage is that the process of capturing and analyzing infrared images can be automated using image processing techniques. Automated algorithms [9] provide a cost efficient way to process large volumes of data. 


\subsection{Characteristics of IRT}

There is no single NDE technique that can be used as a universal replacement for assessing a specimen. Each technique has its own advantages and limitations. Infrared Thermography is being increasingly used in nondestructive evaluation of civil infrastructures because of its benefits.

\subsubsection{Advantages of IRT}

IRT has the following advantages

1. Non-contact : Since infrared camera is used to obtain a thermal image from a distance

2. Ease of Analysis: IRT images are easy to analyze in comparison to other techniques such as GPR images or Ultrasound data.

3. Fast inspection Rate: A single infrared image can cover a large area of the specimen under test. Hence the time required for testing the structure is considerably reduced.

4. Security: No harmful radiation involved.

5. Automation: The entire process of image capture and analysis can be automated, reducing testing time and increasing the ease of operation.

\subsubsection{Limitations of IRT}

Limitations of IRT are:

1. Cost: The cost of thermal equipment such as infrared cameras is high.

2. Depth: Accuracy of infrared thermography results decreases as the depth of the defect increases from the surface of the specimen.

3. Defect size: Small size defects may not be accurately detection by infrared thermography.

4. Thermal Losses: Convective, radiative and conductive losses can introduce errors in the results.

5. Temperature Differential: Enough temperature difference needs to be present to characterize a certain area as defect. Solar radiation may not be 
sufficient to result in such a difference. It is also difficult to uniformly deposit a large amount of energy in a short period of time over a large surface.

\subsection{Measuring Temperature by Infrared Thermography}

All objects above absolute zero $\left(-273^{\circ} \mathrm{C}\right)$ radiates electromagnetic waves in the visible as well as infrared spectrum. The amount of heat radiated is directly proportional to the temperature of the object. The sun at about $6000^{\circ} \mathrm{K}$ appears to glow white-hot. Similarly the color of a hot glowing metal is an indication of its temperature (the higher the temperature, the whiter the color). This method is used in many of the industries to evaluate the product output standard [12]. The electromagnetic waves radiated in the electromagnetic spectrum can be sensed by infrared detectors and converted into electrical signals. A brief explanation to infrared radiation in the electromagnetic spectrum and its conversion into electrical signals used for analysis is given below.

\subsubsection{The Electromagnetic Spectrum}

The electromagnetic spectrum is divided arbitrarily into a number of wavelength regions called bands, distinguished by the methods used to produce and detect the radiation. All bands are governed by the same laws with the basic difference being that of the wavelength in each band [11]. Figure 2.1 shows a graphical representation of the various bands of the electromagnetic spectrum. The various bands mentioned in Figure 2.1 are 1: X-ray; 2: UV; 3: Visible; 4: IR; 5: Microwaves; 6: Radiowaves. The IR band starts at $0.75 \mu \mathrm{m}$ where the band of visible perception ends and the IR band ends at $100 \mu \mathrm{m}$. It is further divided into four smaller bands. They are: the near infrared (0.75-3 $\mu \mathrm{m})$, the middle infrared (3-6 $\mu \mathrm{m})$, the far infrared $(6-15 \mu \mathrm{m})$ and the extreme infrared $(15-100 \mu \mathrm{m})$. The near infrared and far infrared bands are used in most of the infrared cameras to capture a thermal profile. Thus infrared thermography is a non contact, non intrusive means of producing visible images from the invisible heat energy (temperature) emitted from an object. 


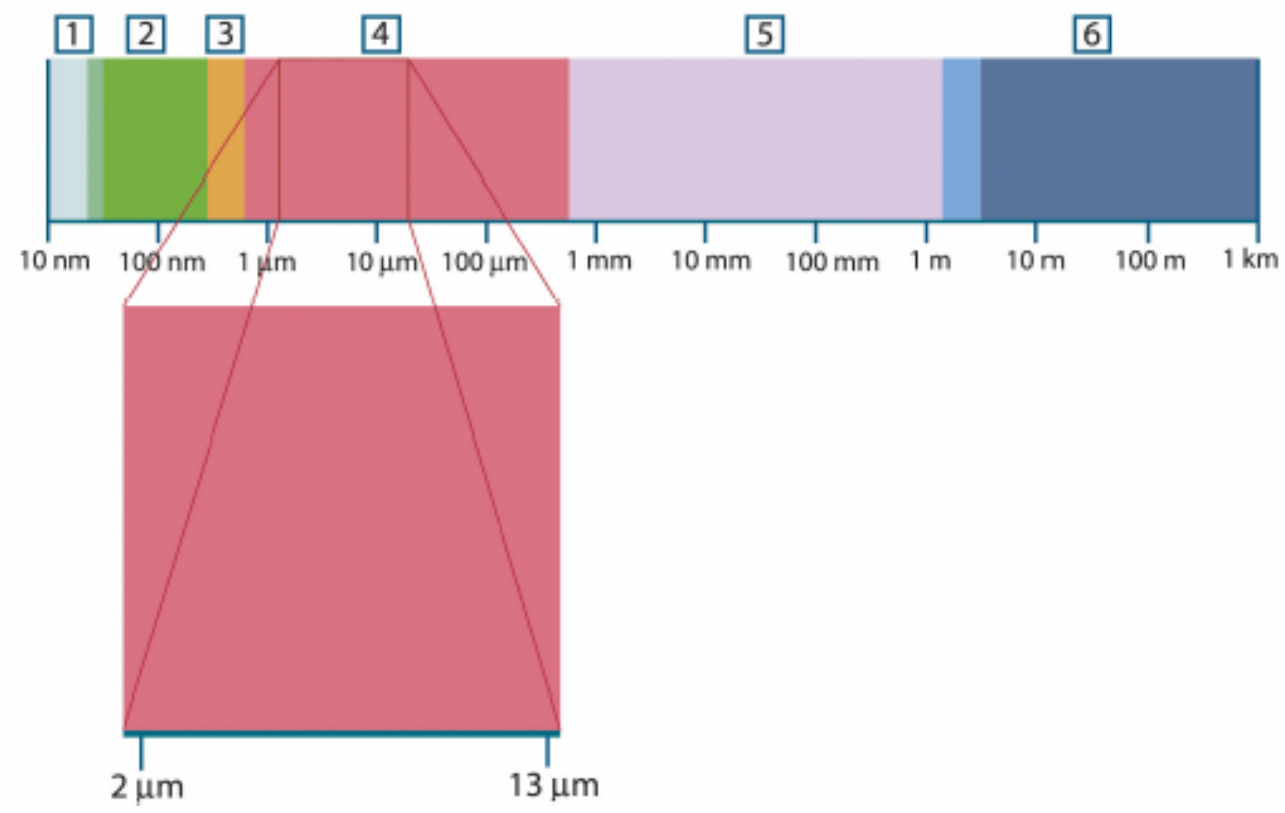

Figure 2.1: The electromagnetic spectrum [11]. 1: X-ray; 2: UV; 3: Visible; 4: IR; 5: Microwaves; 6: Radiowaves.

\subsubsection{Infrared Radiation in the Electromagnetic Spectrum}

Electromagnetic radiation in the infrared band (known as infrared radiation) leaving a surface is called exitance or radiosity. It is emitted from the surface, reflected off the surface or transmitted through the surface. The total radiosity is equal to the sum of the emitted component $(\mathrm{E})$, the reflected component $(\mathrm{R})$ and the transmitted component $(\mathrm{T})$. The surface temperature is related to E, the emitted component only [13]. The emitted component (E) also known as emissivity $(\varepsilon)$ is defined as the ratio of the actual emission from the object to that from a hypothetical source called a "black body" at the same temperature. The values of $\varepsilon$ are between 0 for a perfect reflector and 1 for a perfect emitter, called a 'blackbody'. The three components (E, R, T) cause a flow of heat from one object or place to another. This is known as heat transfer.

\subsubsection{Heat Transfer}

Heat can be defined as the thermal energy in transition. It flows from one place or an object to another because of temperature differences and the flow of heat changes the energy levels in the object [12]. Heat flows from objects at a higher temperature to 


\section{CHAPTER 2. LITERATURE REVIEW}

objects at a lower temperature. Because of heat transfer, hot objects cool down while cool objects tend to become hot. This process continues until an equilibrium is reached. There are three modes of heat transfer namely conduction, convection and radiation [10]. Conduction in a stationery media is the propagation of heat energy from one molecule to another, generally from higher temperature regions to lower temperature regions. The heat flow through a thermally conductive medium is directly proportional to the temperature difference (between hot and cold regions) and the thermal conductivity of the medium. Convection is the heat transfer that takes place due to the motion of a fluid medium (like air) over the solid structural material; for example, between the surrounding air and the deck. The convective heat transfer is determined by the following parameters: the temperature of the solid surface, temperature of the surrounding air and wind speed. Radiation is the heat transfer caused by the emission of electromagnetic waves. It is characteristic of all matter at temperatures higher than absolute zero. The energy transferred due to radiation is directly proportional to the fourth power of the temperature difference between the objects. Thermography measures the temperature differences on the surface of an object by measuring the radiant emission [13].

\subsubsection{Conversion of Infrared Radiation to Temperature}

The advent of modern infrared detectors allows us to measure the temperature of the area exposed to the camera by measuring the energy radiated in the infrared spectrum. Infrared detectors can sense the infrared radiant energy and produce useful electrical signals proportional to the temperature of the objects surface. This is possible by using laws of physics that allow us to convert infrared radiation measurements to temperature measurements. Modern infrared scanners/cameras are capable of measuring temperatures down to sensitivities of $0.05^{\circ} \mathrm{C}$ (or even better with newer generation infrared cameras/imagers) and with response in the microsecond range. They produce thermal maps (or thermograms) with a color or black and white intensity scale which is representative of the temperature of the surface being focused [13]. 


\subsection{Types of Infrared Thermography}

The general scheme of IRT used in NDE applications is shown in Figure 2.2. It shows an infrared camera attached to a visualization tool such as a computer. The infrared camera is focused on the specimen of interest. The specimen may or may not be externally heated as per the requirement. The presence/absence of an external heat source is one method to classify IRT in two schemes known as active or passive IRT. The passive scheme tests materials and structures which are naturally at a different (often higher) temperature. Solar radiation can be used as a natural heat source in case of passive IRT. For example enough solar radiation can cause a substantial temperature difference between the sound and defective area of a bridge deck. In the case of the active scheme, an external stimulus is necessary to induce a relevant thermal contrast that is not available otherwise. e.g. specimen at uniform temperature prior to testing [14].

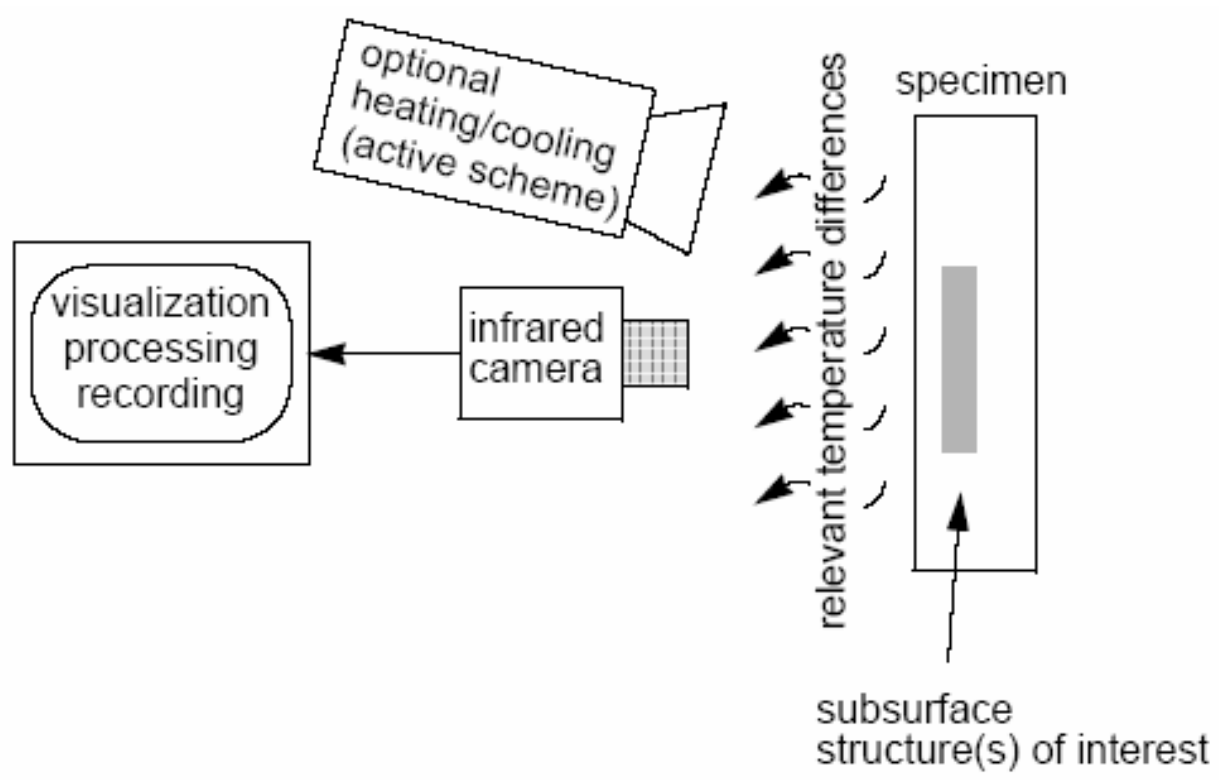

Figure 2.2: General setup of infrared thermography used in NDE [14].

In many industrial processes temperature is an essential parameter to assess proper operation and passive thermography aims at such measurement. Important applications of the passive approach are in production, predictive maintenance, medicine, 


\section{CHAPTER 2. LITERATURE REVIEW}

fire forest detection, building thermal efficiency survey programs, road traffic monitoring, agriculture and biology, medicine, detection of gas (by mean of absorbing tracer gas) and in NDE [15]. In all these applications, abnormal temperature profiles indicate a potential problem that needs to be taken care of. For example the presence of an anomaly such as an air void below the surface of a bridge deck disrupts normal heat transfer process. Hence a high temperature area is formed on the surface of the bridge deck above the location of the anomaly. The actual temperature difference between the sound and the defective area is dependent on many factors such as transmissivity of the medium, air flow, solar radiation etc. A difference of approximately $4{ }^{\circ} \mathrm{C}$ can be positively identified as an anomaly/defect. On the other hand a temperature difference of $2^{\circ} \mathrm{C}$ may or may not be classified as a defect.

The active approach requires an external stimulus such as an external heater, to generate relevant temperature differences not present otherwise. User control of this external stimulus (example: time $t_{0}$ when it is applied) enables quantitative characterization such as the depth of a detected disbond to be measured. Depending on the external stimulus, different approaches of active thermography have been developed such as pulse thermography (PT), step heating (SH), lockin thermography (LT), vibrothermography (VT) etc. These methods are discussed in detail by $[13,14,15]$ among other references. The active approach finds numerous applications in NDE.

\subsection{Applications of IRT}

Applications for infrared thermal sensing and imaging are found in many industries. A common way to characterize most of the applications of IRT is its use in increasing the reliability of a certain piece of equipment. Reliability is defined as "the probability that a certain piece of equipment will operate in a given environment, within given performance limits and for a certain length of time." A reliability of $1(100 \%)$ is possible only with a perfect piece of equipment. Since $100 \%$ perfection does not exist in this world, this figure is never obtained [16]. IRT is one of the tools that is used to assess the real extent of stresses active in the component, so that reliability figure approaches closer to 1 . Some of the applications of IRT are briefly described below. 


\subsubsection{Plant Condition Monitoring and Maintenance}

Maintenance through periodic monitoring is one of the prominent applications of IRT, especially where abnormal temperature profiles indicate a potential problem. IRT is being increasingly used since more than 25 years for plant condition monitoring and preventing field maintenance. It has been universally accepted in the power industry where huge amount of thermography data from power line surveys has been collected and standards have been developed for thermal behaviour of electrical switchgear and distribution equipment. IRT is extensively used to reduce the risk of failure in the power generation industry including nuclear power plants. They conduct some of the most comprehensive predictive maintenance programs using infrared thermography.

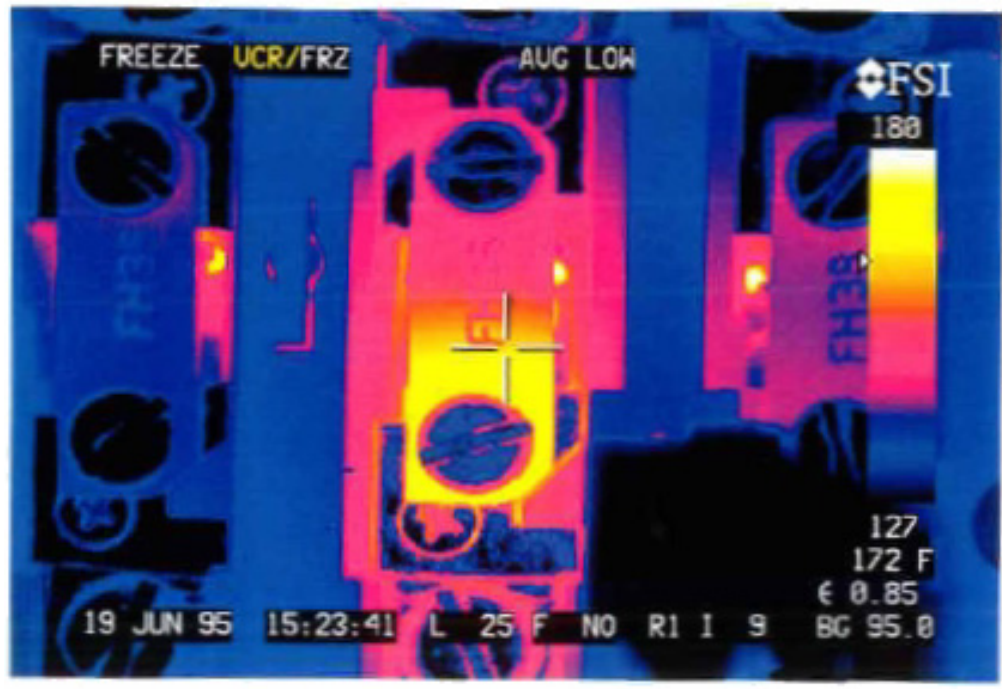

Figure 2.3: Infrared image for excessive heating of a connecting clip [13].

Another area is using IRT to detect anomalies in electrical facilities and utilities. Most common electrical findings such as short circuits, open circuits, inductive currents, high electrical resistance etc are detected using IRT. These anomalies can harm the electrical equipment, cause power loss and reduce their working life if they are not detected in the earliest possible stages. Figure 2.3 shows such an application where IRT is used to detect deterioration of a connection clip caused by excessive heating due to high resistance of the clip. Other applications of IRT include monitoring quality of production in the paper industry, friction monitoring in metal industry, pipe blockage detection etc. 


\subsubsection{Buildings and Infrastructure}

The use of IRT to detect energy losses in structures was one of the first commercial applications of IRT. IRT is being used since 1970 both by the government and other communities to gather information regarding the heat loss characteristics of commercial, residential and government buildings. Measurement of insulation efficiency and characteristics of structures is another application of IRT. Another industrial application of IRT is roof moisture detection. Moisture from leaky roof may affect the roof structurally as well as functionally. The penetration of moisture causes degradation in the reinforcing steel. Research done by [17] discusses the thermal modelling of a leaky roof and its effects using IRT as a tool for measuring the energy loss. Commercial applications of moisture detection are also prominent.

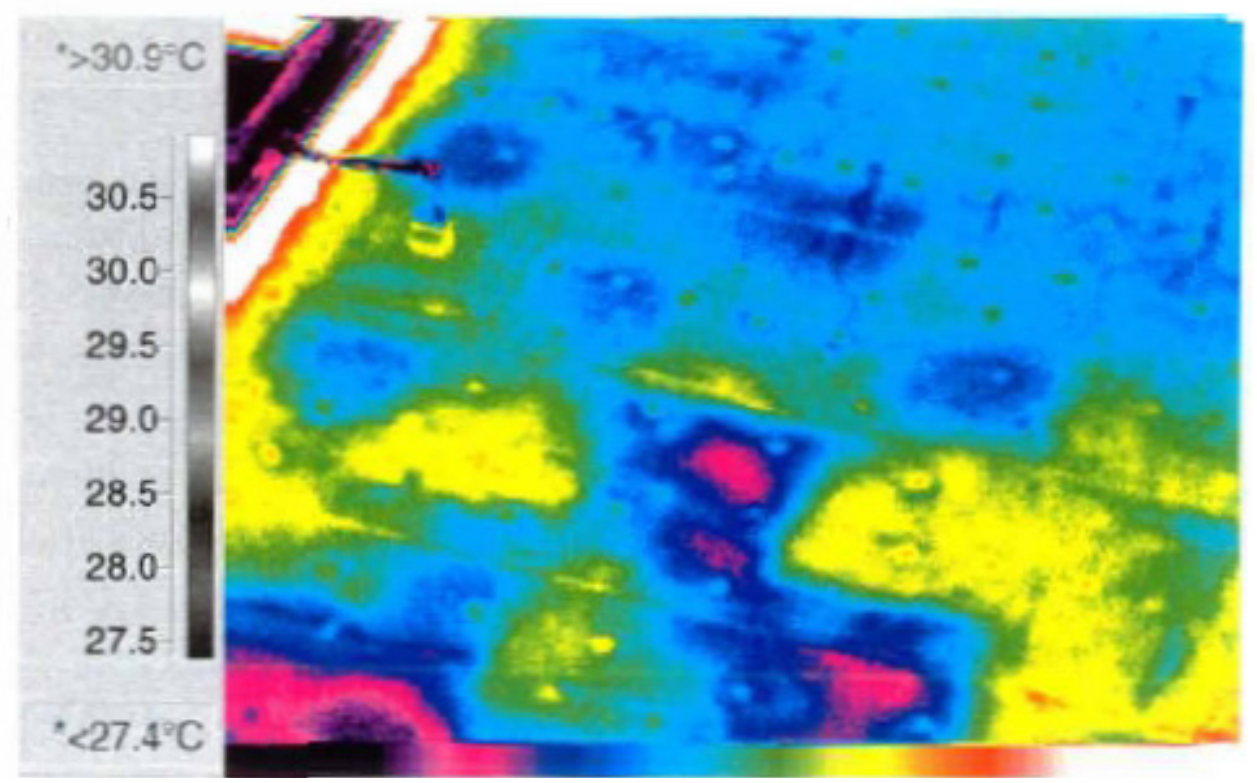

Figure 2.4: Thermogram of a roof with moisture saturation [13].

Figure 2.4 shows a thermal image of a roof captured by Infrared Thermal Imaging Corp. The thermogram of the roof shows areas with moisture saturation that can cause structural deterioration. Testing or evaluating the integrity of other structures such as bridges by IRT is also in widespread use. This thesis explores such an application. 


\section{CHAPTER 2. LITERATURE REVIEW}

\subsubsection{Production Testing and Monitoring}

Materials in day to day use can be tested either during production or during their life of operation by using IRT. This includes materials such as cables, wires, resistors, printed circuit boards etc. Testing of materials during production has the advantage of testing in a closed and controlled environment. The amount of active heating can be controlled, errors due to flow of air, component movement, irregular exposure to heat etc., can be minimized. This leads to early detection of faulty components and increase in the reliability of the defect free components. Various approaches to such a controlled defect detection system have been developed. Some of them are discussed in detail in [13]. Automatic defect detection systems can be developed with high accuracy of defect detection in such a scenario.

\subsubsection{Security and Surveillance}

The military were among the first to envisage the use of IRT for detection of potential targets in low light conditions. A lot of research is being done to investigate the modelling and prediction of target signatures in various conditions. Another application is using IRT to detect incoming missiles from their hot gas exhaust. IRT has also found applications in surveillance and night vision. Modern police cruises are outfitted with low cost portable night vision systems that are effective in crime interdiction. The same technology is used in helicopters to aid pursuit of suspected offenders. Recently night vision technology is being used as an aid for drivers in low visibility conditions. This will provide drivers with enhanced vision through smoke, fog, and extend the vision of drivers to see other objects.

IRT is a powerful tool with applications in many industries. Recent developments in technology of infrared camera have enabled accurate measurement of temperature as well as thermal image capture in a wide variety of scenarios. The digital revolution coupled with high speed processing capability facilitates the processing of large amounts of data with high reliability. Thus a thermal data acquisition and processing system can be built to suit the requirements of an application. 


\subsection{Infrared Thermography for Defect Detection}

Repair and strengthening of existing structures and building new ones with bonded Fiber Reinforced Polymer (FRP) composites are rapidly gaining acceptance. The long-term durability aspects of FRP are still unclear. Research and development work is being conducted for nondestructive evaluation of Glass Fiber Reinforced Polymer (GFRP) composite members using Digital Infrared Thermography. A study conducted by Alqennah [18], among others investigated the use of infrared thermography for detection of debonds and delaminations in FRP decks. Four composite deck specimens (Sample1, 2, 3 and 4) having delaminations of different sizes, filler material and varying locations from the edges were inserted to study the effects of these variables on the infrared nondestructive testing technique. A 3/8" thick wearing surface was used on top of the FRP decks. The cross-section of the specimen is as shown in Figure 2.5.

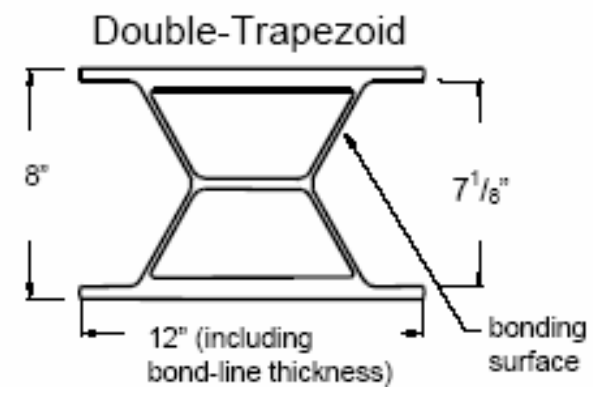

Figure 2.5: The cross section of the composite bridge deck specimen [10, 18].

Two infrared cameras, Insight 80 Series and the FLIR Prism Single Point camera with a resolution of $0.1^{\circ}$ and $0.2^{\circ} \mathrm{C}$, respectively, were used to capture a temperature profile of the area under test. Sample 1 was tested using both the cameras and neither could detect the simulated debonding as seen in Figure 2.6(b). Similarly, sample 2 that had several built in delaminations was heated to different temperatures ranging from $20^{\circ} \mathrm{C}$ to $80^{\circ} \mathrm{C}$ using different heating sources but no infrared image was able to show the delaminations. 


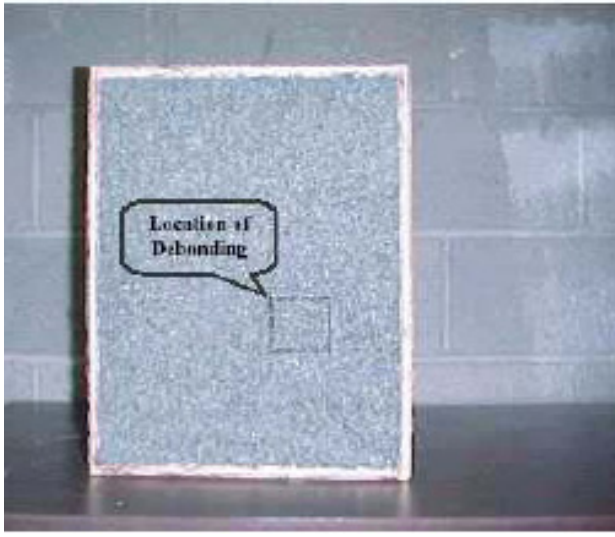

(a)

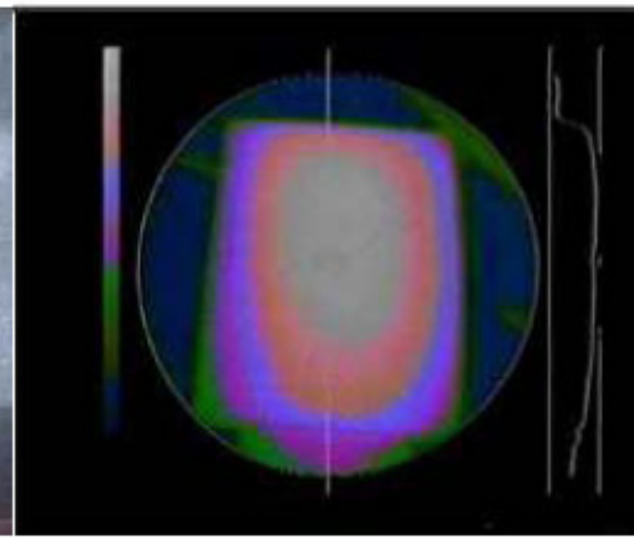

(b)

Figure 2.6: (a) Photograph, and (b) Infrared image of Sample 1 [18].

The delaminations in sample 3 and sample 4 were clearly visible in the infrared image. An example is shown in Figure 2.7 that shows the schematic of defects in sample 4 and the corresponding infrared image. Good contrasts between the debonded and the defect free area were achieved and the delaminations were seen clearly.

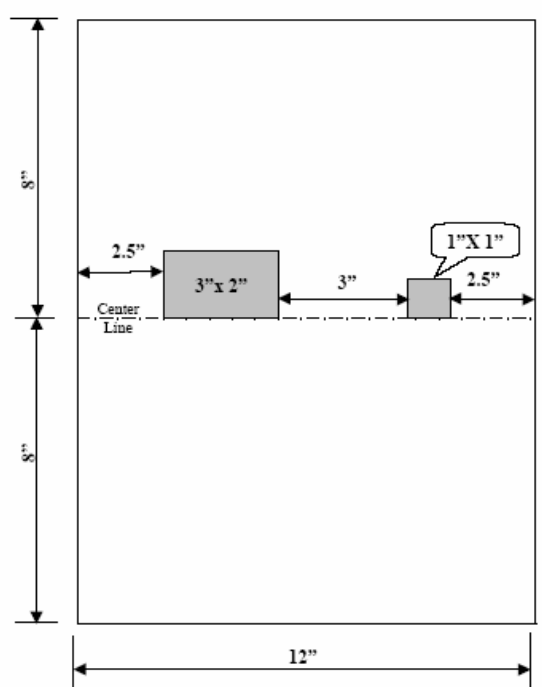

(a)

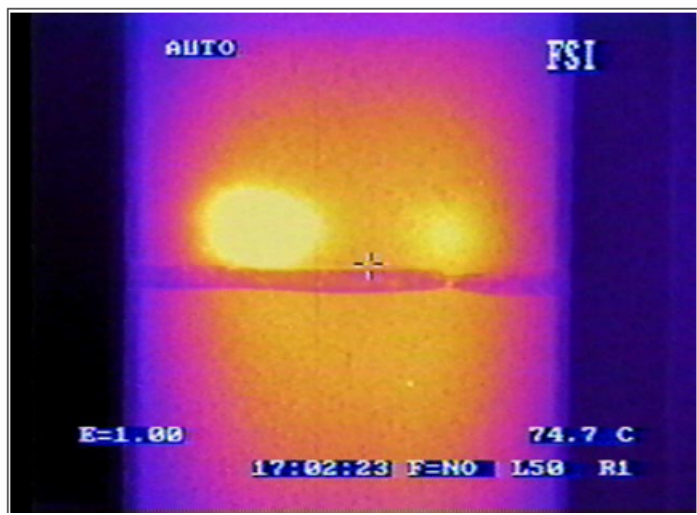

(b)

Figure 2.7: (a) Schematic of the FRP deck Sample (b) Infrared image of the Sample [18].

It was concluded from the laboratory experiments that infrared thermography is able to successfully detect subsurface delaminations under a wearing surface. The study 
was extended for the condition assessment of two composite bridges (Laurel Lick bridge and Wickwire Run bridge) using infrared thermography. Results indicate that the relatively new Laurel Lick and the Wickwire Run bridges (constructed in 1997) did not have any subsurface delaminations. However, some infrared images showed surface temperature differentials on certain portions of the bridge deck caused due to the shadows from the guard rails, surface irregularities and gravel. Figure 2.8 shows as infrared images of the Laurel Lick bridge deck with surface irregularities caused by guardrail.

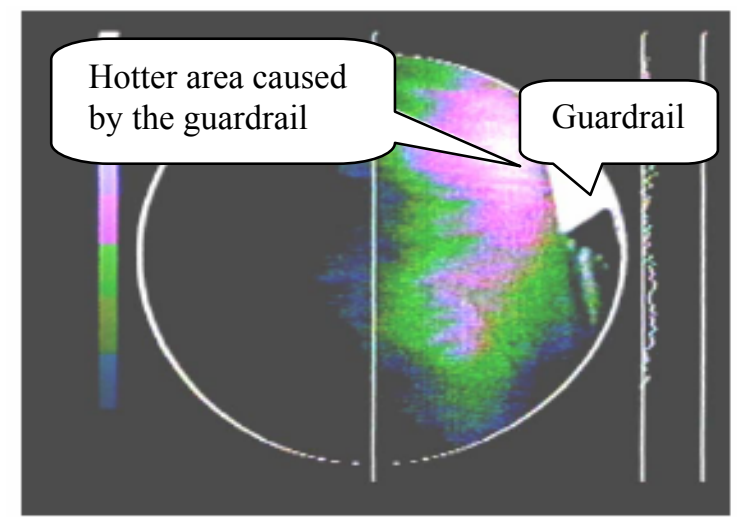

(a)

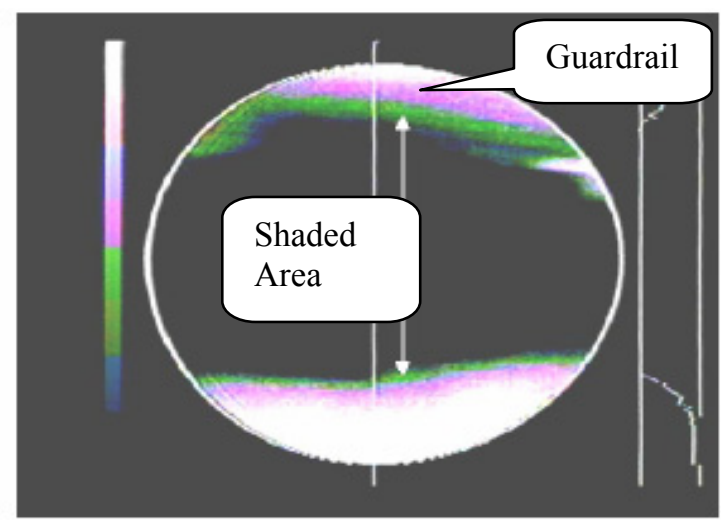

(b)

Figure 2.8: Infrared images showing (a) hotter areas caused due to reflection of solar rays by the guard rails, and (b) colder areas caused due to shadows from guard rails [18].

The field tests conducted on the two composite bridge decks led to the conclusion that both the bridges did not have any subsurface debonds. It was observed that reflections from the guard rails, shadows of the guard rails, gravel and other surface irregularities caused some temperature differentials. These were correctly detected by Infrared Thermography and could be misinterpreted by the user as an anomaly. Hence it was concluded that care must be exercised when interpreting these images.

The application of active heating through lamps, convective air etc causes a variation in thermal pattern. These variations can be used as indicators for possible structural deficiencies. This has been investigated by Miceli 2000 [19]. The study was conducted on a model bridge deck constructed with components from the EXTREN® System by Strongwell Corp. First, infrared thermography was selected as an NDE method to efficiently evaluate the given material. Then the capabilities of that method 
were assessed for detection of simulated damage states and environmental conditions. Finally, preliminary fieldwork on full-scale bridge decks was performed.

The research presented in this work focuses on a smaller model of the large deck system. The deck consists of 4 tubes and two plates adhesively bonded with Magnabond. Two holes were drilled and the tubes were sanded together followed by bonding them to the flat panel using an adhesive. After the tubes were cured as a panel, debonds were simulated on the deck. There were two debonds purposely placed in the deck. The debond between the top plate and the tubes had a small debonded channel leading to an opening in the side of the deck. This was used to fill the debond with water. A Raytheon Amber Radiance 1T image system with an Indium Antimonide detector array and a temperature range of $-20^{\circ} \mathrm{C}$ to $300^{\circ} \mathrm{C}$ was used for thermal imaging. Forced hot air through the tubes was used to heat the whole length of the tube. Since the disbonds disrupt the heat conduction, they could be seen in the thermal images.

First a baseline thermal pattern was established for each of the undamaged tubes. The formation of such a baseline thermal pattern is shown in Figure 2.9(a) and the completely formed thermal pattern after 8 minutes of cooling is shown in Figure 2.9(b).

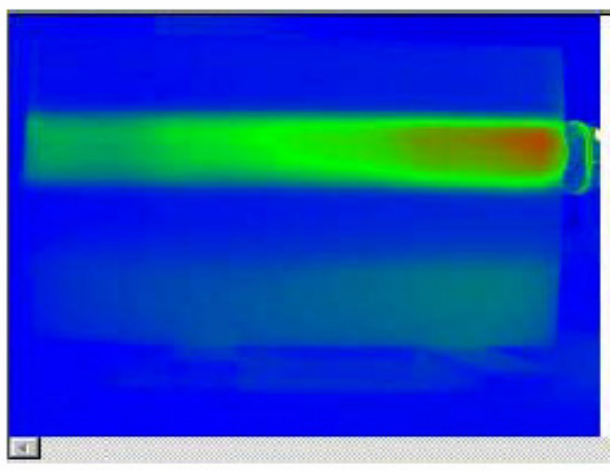

(a)

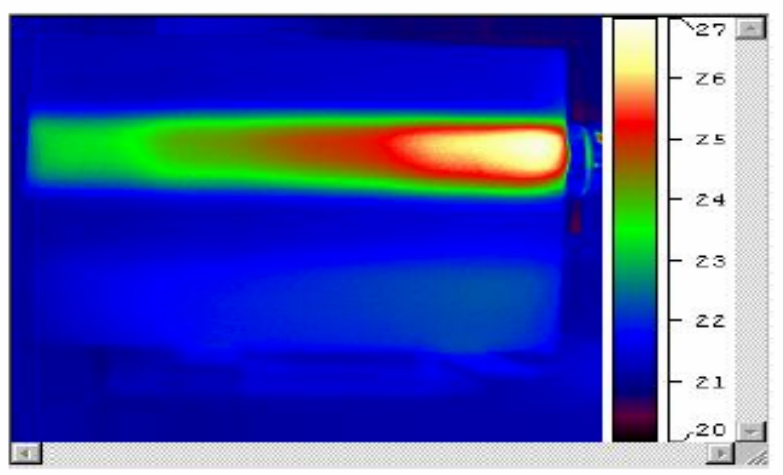

(b)

Figure 2.9: (a) Tube \#3 after 4 minutes of cooling. Time dependent boundary layer thermal pattern begins to appear. (b) - Tube \#3 after 8 minutes of cooling. Fully developed thermal pattern shown [19].

The next test series examined the temperature of the back surface of the model deck. This series was performed in a similar manner to Test Series \#1. Each tube was 
investigated individually for anomalies in the thermal pattern. Abnormal thermal patterns were observed under different conditions. One of the results for tube 2 with a water debond and no wearing surface is shown in Figure 2.10(b)

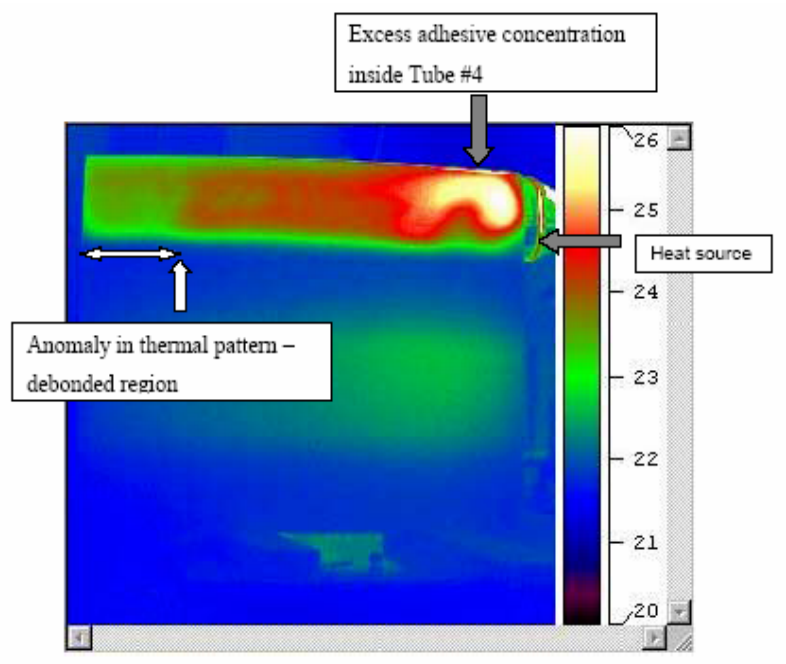

(a)

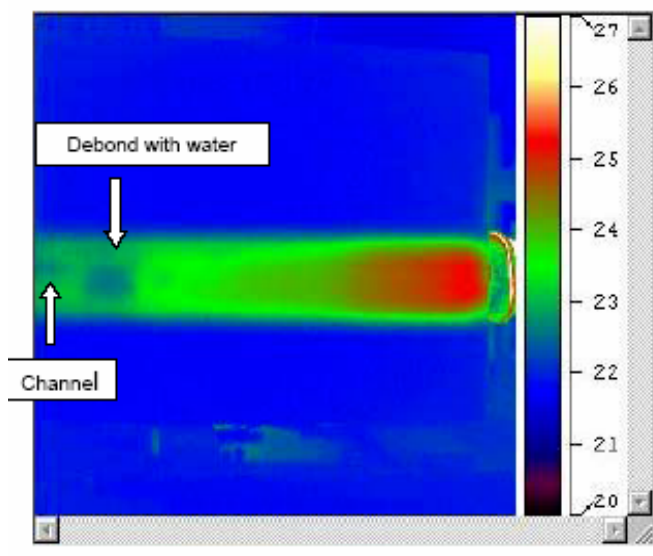

(b)

Figure 2.10: (a)Tube \# 4 after 4 minutes of heating and 4 minutes of cooling. (b)Tube \#2 with water in debond and no wear surface [19].

Further efforts to define the sensitivity of the method to disbond type imperfections were pursued using model deck in the laboratory. The model deck was tested using forced hot air. Mathematical modeling of the heat conduction associated with the convective heat transfer was in close agreement with experimental observations. It was found that infrared thermal imaging is a suitable technique for monitoring the health of FRP structures by detecting the possible presence of debonds and delaminations under various conditions. Detection accuracy and resolution restrictions for the field testing were found to be $7.8 \mathrm{~mm} / \mathrm{pixel}(0.31 \mathrm{in} / \mathrm{pixel})$ at $5160 \mathrm{~mm}$ (16.9in) from the edge of the deck. It was observed that environmental factors can cause a decrease the accuracy of estimation of debonds by IRT.

Continued research has being done at West Virginia University by [10, 12, 21, 23] to evaluate the use of GFRP decks, for building new structures as well as for reinforcing old ones. The research by Banglore [12] established the effectiveness of thermography as a tool for nondestructive evaluation of FRP composite materials. The experiments 


\section{CHAPTER 2. LITERATURE REVIEW}

consisted of two steps. The first step was the creation of the delaminations of known shapes and sizes in the specimens and the second was to locate them using infrared thermography. The schematic of the setup for the research is shown in Figure 2.11(a) and the photograph of the same is shown in Figure 2.11(b).

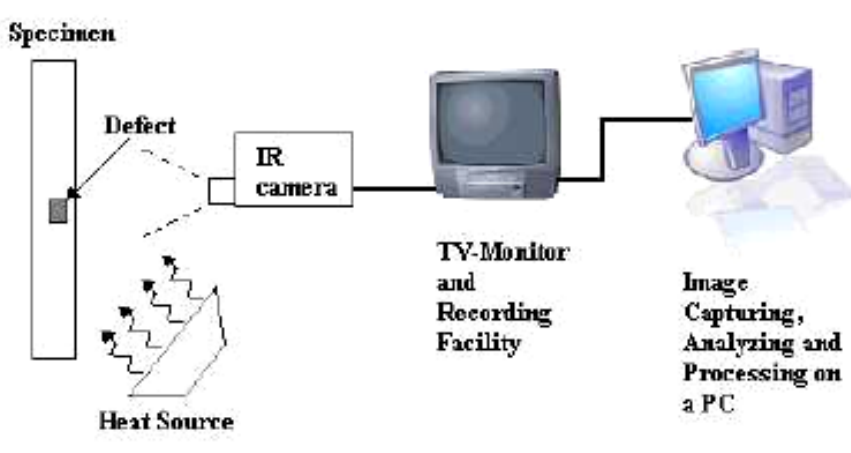

(a)

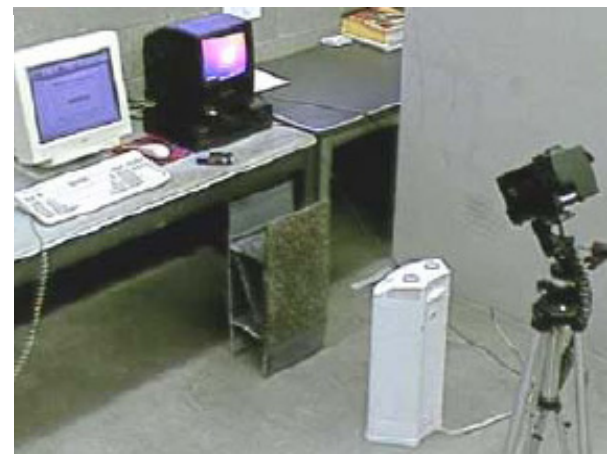

(b)

Figure 2.11: (a) Schematic view of the infrared thermography set up in the laboratory

(b) Experimental setup [12].

The thermal images were recorded using a FSI Prism SP (Single Point) infrared camera were recorded on a VHS tape and later transferred as still images into a personal computer. The heating source used in the laboratory was a quartz tower heater. Two types of glass fiber reinforced polymer (GFRP) composite sections were tested in the laboratory. They were 2"x5" box sections and bridge deck specimens. The delaminations of different dimensions were inserted in these box sections. Some of the sections had surface delaminations of 3"x3" and 2"x2". One of the box sections was surface painted so that the delamination was not visible. The specimens along with the details of the delaminations in each are shown in Figure 2.12. The second type of specimens was a FRP bridge decks that was 24" wide, 8" deep and 12 feet long (pultruded at Bedford Reinforced Plastics Inc., PA.). Two specimens named BD1 and BD2 were prepared. Specimen BD1 had two delaminations of sizes 3"x3"x1/16" and 2"x2"x1/16", that were inserted within the joint between the two modules. 


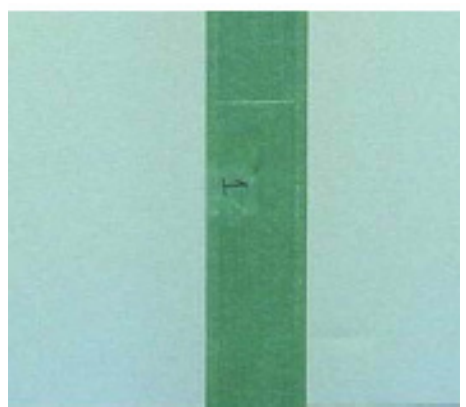

(a)

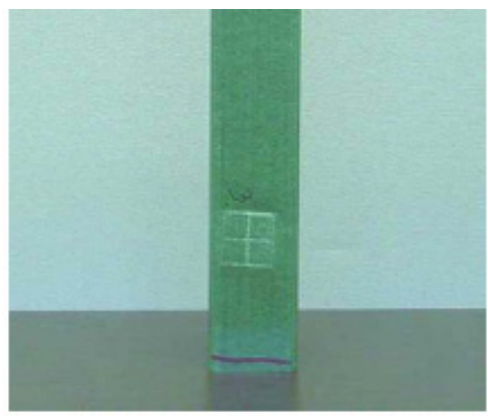

(b)

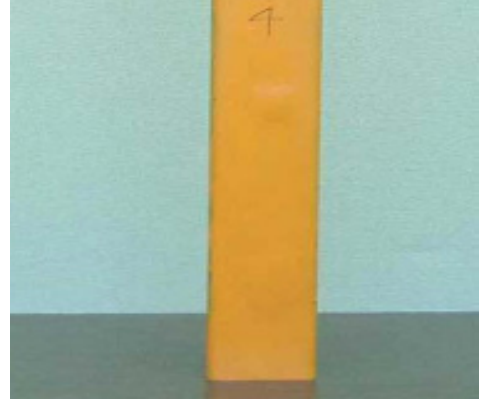

(c)

Figure 2.12: Box section specimens (a) Box1: 2"x2" delamination with 1/16" thickness (b)Box3: 3"x3" delamination with 1/16" thickness (c)Box4: one 3"x3" and another 2"x2" delamination with 1/16" thickness [12].

The specimens were heated by placing the quartz heater at a distance of 6-8 inches from the surface. The surface temperature profiles were observed using the infrared camera and were recorded on a VHS tape. Figure 2.13(a) and (b) show infrared images in the box sections. The image in Figure 2.13(a) is that of Box3 with a 3"x3" delamination. It is clearly seen as a high temperature area in the image. Similarly Figure 2.13(b) is an infrared image of Box4 that has 3"x3" and 2"x2" delaminations with paint on the surface. Both the 2"x2" and 3"x3" delaminations are clearly visible.

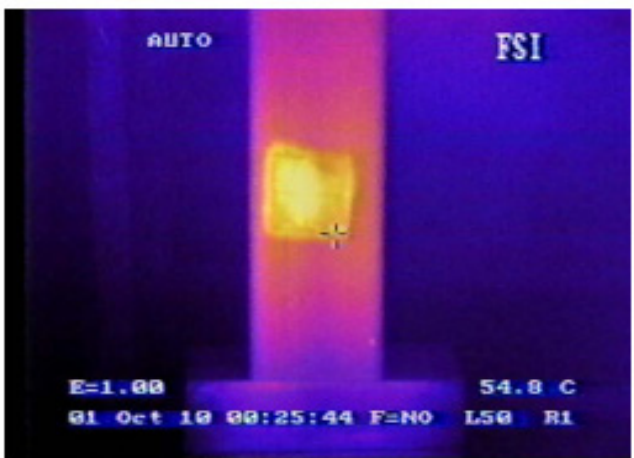

(a)

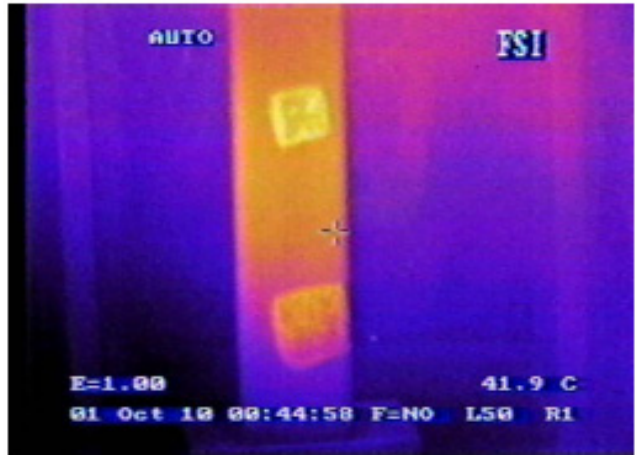

(b)

Figure 2.13: Infrared images of specimen Box3 and Box4 (a) Specimen Box3 color infrared image with observed delamination. (b) Specimen Box4 color infrared image with observed delamination [12].

The infrared images of bridge deck specimens BD1 and BD2 are shown in Figure 2.14(a) and (b). The infrared image of BD1 (Figure 2.14(a)) showed some differential 
temperature zones, but the shapes of the patches were different from what was expected and hence could not be categorically judged as delaminations. On the other hand Figure 2.14(b) shows infrared image of deck BD2 with the debonds (seen as bright yellow/white regions) of sizes 3"x3" and 2"x2" present in between the 3/8" thick wearing surface and the underlying deck. Infrared thermography was very successful in predicting the locations as well as the shapes and relative sizes of the two debonds.

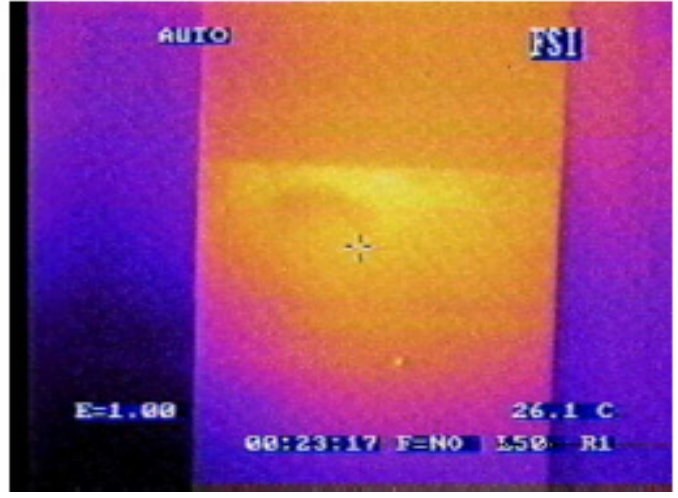

(a)

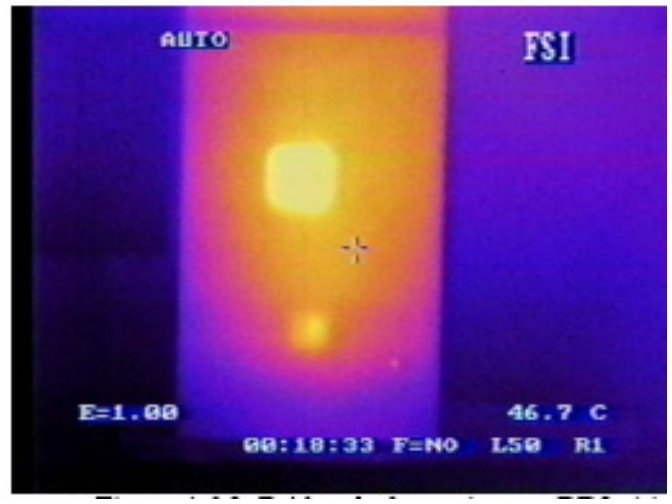

(b)

Figure 2.14: Infrared images of bridge deck specimens (a) BD1 (b) BD2 [12].

The results from the wide range of tests led to the conclusion that infrared thermography can be used as an effective tool for detecting subsurface delaminations in members built with FRP material. The delaminations present between the flanges of two adjacent sections in the bridge deck specimen BD1 could not be located with the current infrared technique. However, the delaminations between the wearing surface and the bridge deck surface in specimen BD2 could be easily detected. The extension of this research was further carried out by Vasudevan et. al. [10, 21] and Roy et. al. [4, 22]. Vasudevan's research was concentrated on finding the effect of various imaging conditions such as changing the distance between bridge deck and the camera, changing heating conditions etc. The research by Roy [4, 22] was focused on using infrared thermography to locate water filled delaminations and debonds under varying condition. The setup for the research is similar to the one shown in Figure 2.11. A FLIR ThermaCAM ${ }^{\mathrm{TM}}$ S60 digital infrared camera was used to capture the thermal image. A 
thermal gradient was established using a heating or cooling source. A laptop computer connected to the infrared camera was used to store the sequence of digital images captured by the camera.

The bridge deck specimen used consisted of GFRP bridge deck module of various sizes. Bridge deck named BD1 consisted of plan size 2'x1' with overall thickness of 8". The deck was coated with a wearing surface layer of thickness $3 / 8$ ". The front and cross sectional view of the deck is shown in Figure 2.15(a). Debonds of size 2"x2" and 3" x 3" were placed on one side. The other side consisted of debonds of sizes 1 " $\mathrm{x} 1$ " and $1 / 2$ " $\mathrm{x} 1 / 2$ " placed between the wearing surface and the deck. Figure 2.15(b) shows the location of debonds on both the sides of the deck. Bride deck specimens similar to BD1 were created by the same process. They differed in the size of debonds, use of wearing surface etc. For example, deck BD2 had two water-filled debonds of sizes 2"x2" and 3"x3" and an airfilled debond of size 2"x2".

Three bridge decks named JD1, JD2 and JD3 with simulated delaminations of different sizes were also prepared as a part of this research. They were low profile GFRP bridge decks similar to either BD1 or BD2 that were described above. Infrared images of these bridge deck specimens subjected to active heating were captured by the infrared camera. The infrared images of the specimen revealed the location of the defects as white regions in case of air filled defects and black region in case of water filled defects.

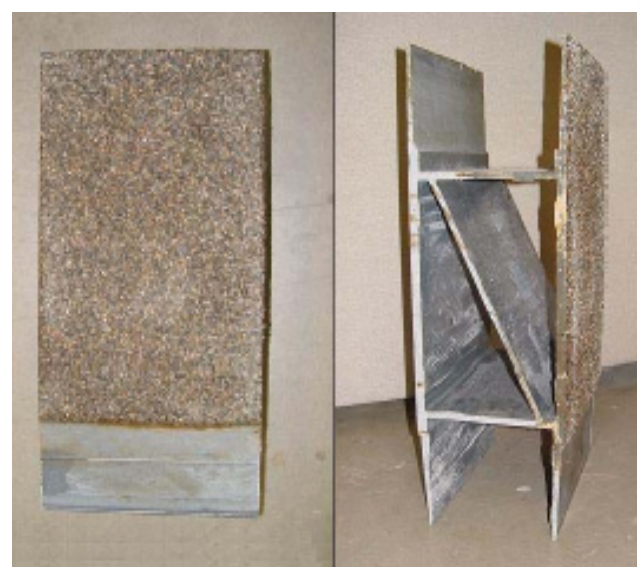

(a)

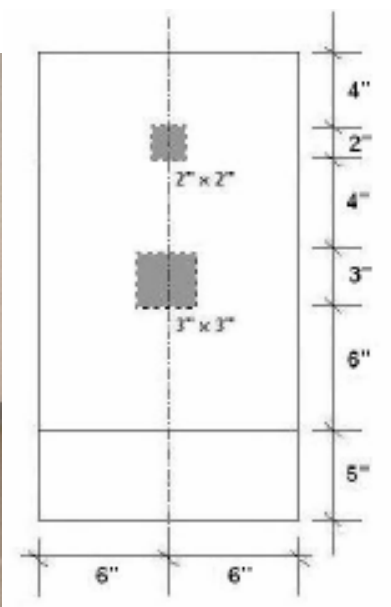

(b)

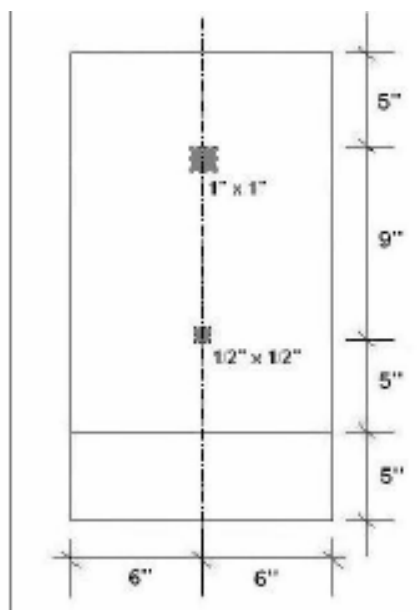

Figure 2.15: (a) Front view and cross-sectional view of the GFRP bridge deck specimen BD1 with w/s (b) Schematic view of location of debonds in bridge deck specimens (a) BD1a (b) BD1b [10]. 
Figure 2.16(a) shows the infrared image of the 2"x2" and 3"x3" debonds in specimen BDla. The boundaries of both these debonds were well-defined in the image with temperature differential between the defect and the defect free area of $11^{\circ} \mathrm{C}$ and $8{ }^{\circ} \mathrm{C}$ respectively. A smaller temperature differential was obtained for the 1 " $\mathrm{x} 1$ " and $1 / 2$ " $\mathrm{x} \frac{1}{2}$ " defects in BD1b.

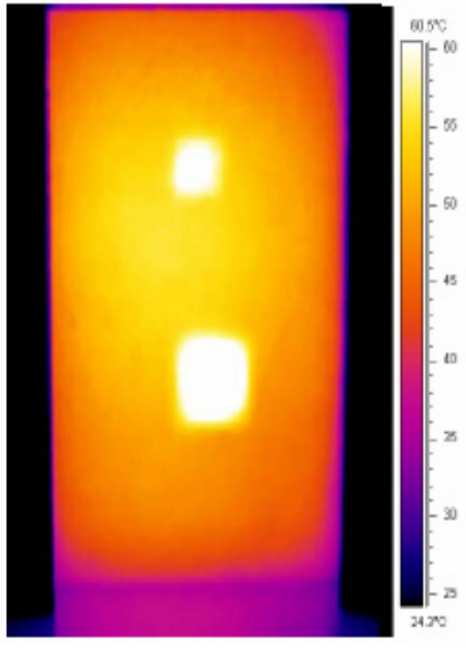

(a)

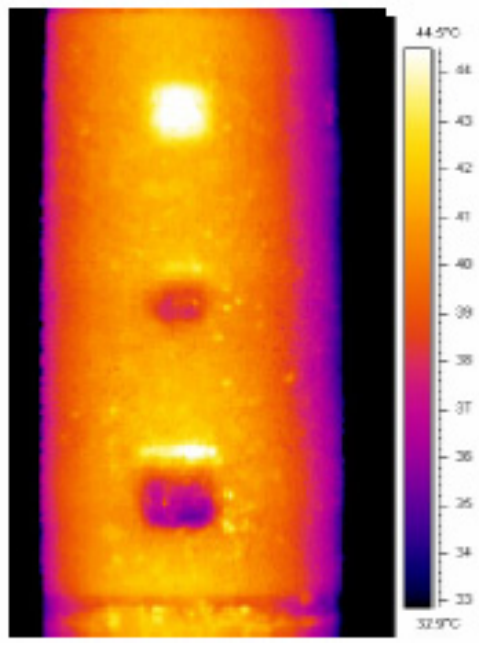

(b)

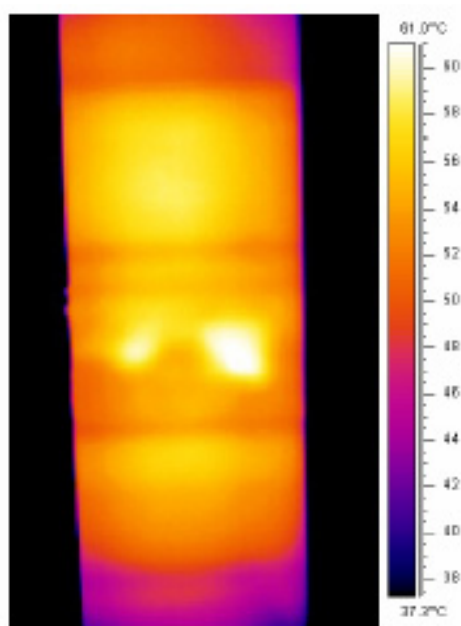

(c)

Figure 2.16: Infrared images of Bridge deck BD1 and BD2 (a) Infrared image of BD1a with debonds of sizes 2"x2" and 3"x3" (b) Infrared image of deck BD2 (c) JD1a with 3"x3" delamination (b) JD1b with the 1"x1" and 2"x2" delaminations [10].

Similarly infrared image of BD2 is shown in Figure 2.16(b). The water filled debonds of size 3"x3" and 2"x2" in BD1 were also detected. They appear as cold spots because of high thermal conductivity of water. Delaminations in JD2 (1"x1" and 2"x2") were detected as seen in Figure 2.16(c). More heat was required to recognize the delaminations. Boundary of detection of the delaminations was not accurate. From all the tests conducted to detect both debonds and delaminations, the digital infrared thermography has satisfied the standards specified in ACI 440.2R [10].

Tests under solar radiation showed that solar radiation can act as an uneven heat source. Figure 2.17 shows the infrared images of the bridge deck JDla taken during different times of the day. The temperature difference for this delamination was found to be about $4^{\circ} \mathrm{C}$ at 2.30 P.M. and is clearly seen in Figure $2.17(\mathrm{c})$ as a white square region. It 
was observed that false defects can be caused because of uneven heating by the sun as seen in Figure 2.17(a).

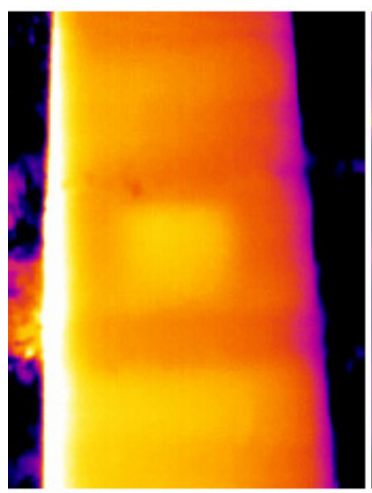

(a)

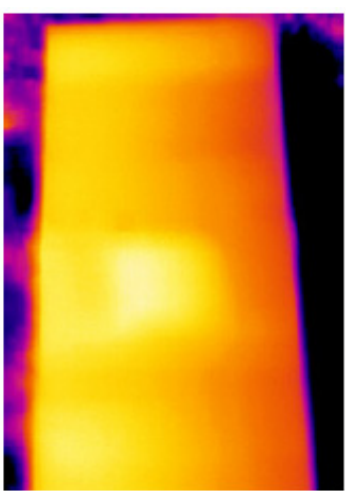

(b)

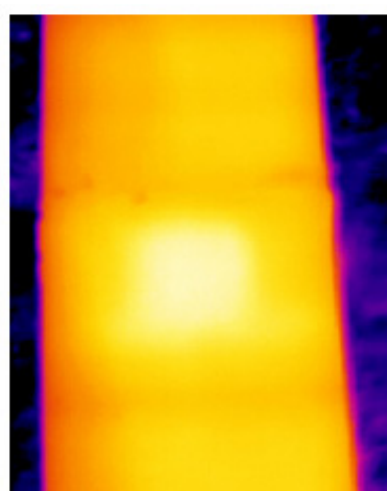

(c)

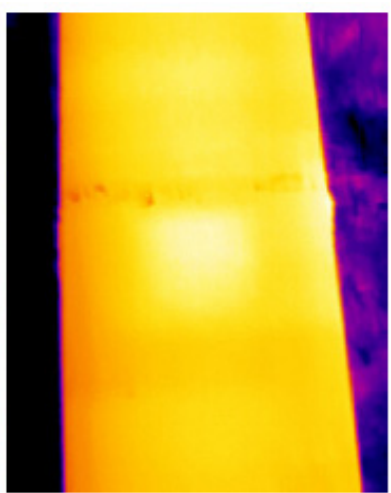

(d)

Figure 2.17: Infrared image of bridge deck specimen JD1a with delamination of size 3"x3" at (a) 9.30 A.M. (b) 12.30 P.M. (c) 2.30 P.M. (d) 4.30 P.M [10].

Similar experiments on FRP deck specimens having water filled debonds and delaminations were conducted by Roy [4, 22]. In this case, the specimens were first heated by the quartz tower heater. For a specimen having a wearing surface, it was heated to around $85^{\circ} \mathrm{C}$ for 8 to 10 minutes since it took a long time for the heat to penetrate to the level of the defect embedded 2" below the wearing surface. The infrared image in Figure 2.18(a) shows a patch of high temperature region around the 4" $\mathrm{x} 4$ " debond but the 2"x2" debond could not be seen.

Figure 2.18(b) shows an infrared image of specimen with detected debonds. The infrared image did not show any clear boundary between the defective regions in both the specimens. A noticeable temperature difference of approximately $8^{\circ} \mathrm{C}$ was needed for identifying the debonds. Specimens WJD1,2,3 had to be heated for a longer time so that the heat penetrated the FRP layers and reached the delamination. Hot spots in the infrared image indicated the presence of air. The 1"x1"x1/16" delamination in WDJ1 could not be detected at all and the 2"x2"x1/16" delamination was observed as a cold spot but with a very poor contrast as is clearly seen in Figure 2.19(a). 


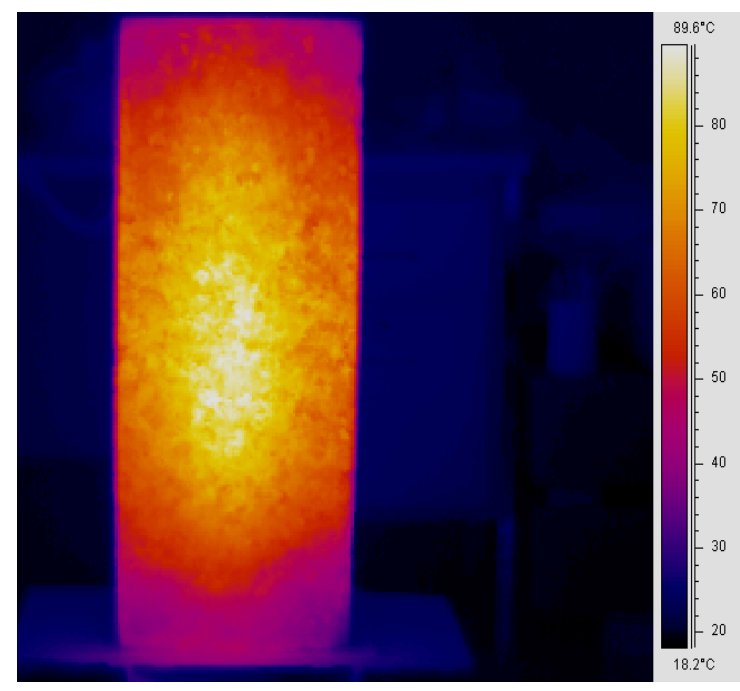

(a)

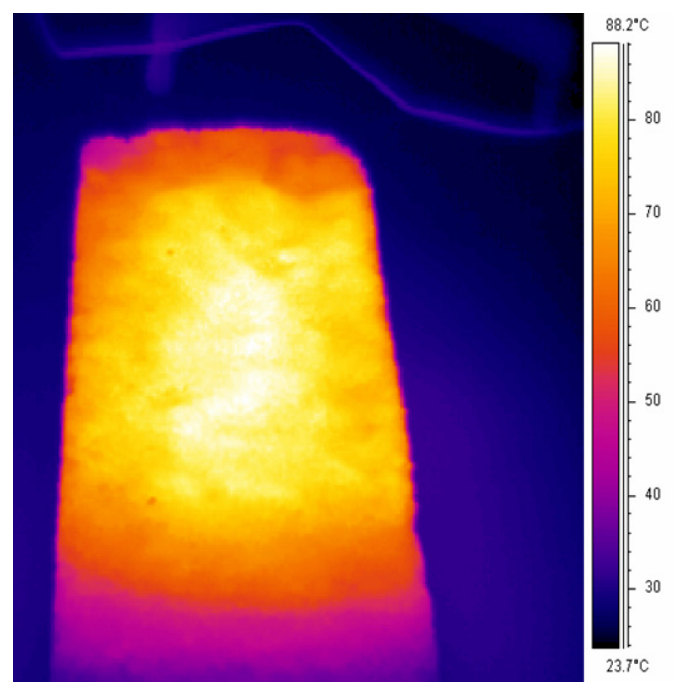

(b)

Figure 2.18: (a) Infrared image of the 2"x2" and 4"x4" debonds in specimen ASP1 with 2" wearing surface (b) Infrared image of 3"x3" air-filled debond in ASP2 heated with the shop heater in horizontal position [4].

Figure 2.19(b) shows a close up of the infrared image of the specimen WJD2 with the 3"x3"x1/16". The water-filled delaminations are seen as dark square regions. A temperature difference of about $-6^{\circ} \mathrm{C}$ was sufficient to identify the defect with an average temperature of about $65^{\circ} \mathrm{C}$ after 1 minute of removal of the heat source. The experiments and resulting observations from the research by $[4,10,21,22]$ show that infrared thermography is successful in delineating subsurface defects in FRP bridge decks under various conditions. The accuracy of defect detection depends on many factors such as size of the defect, depth of the defect from the surface, distance between camera and bridge deck etc. It was also observed that though solar radiation can be used as a passive heat source, it may affect the accuracy of defect detection.

The results from infrared thermography were found to satisfy the available standards for inspection method used for detecting delaminations in FRP systems, which states that the method should be capable of detecting delamination of 2 " 2 " or greater. 


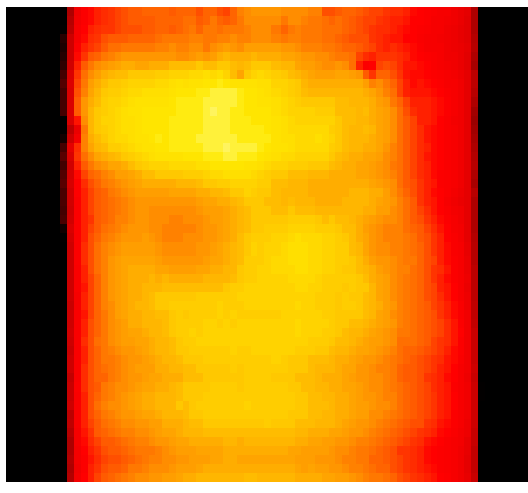

(a)

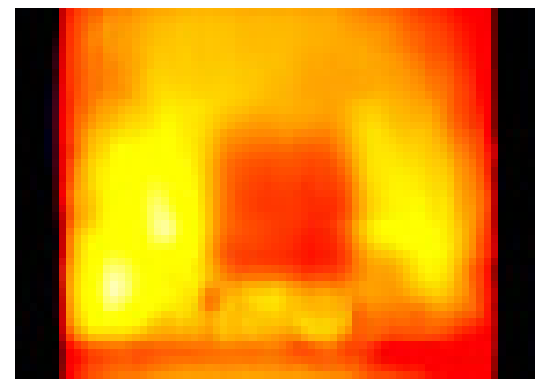

(b)

Figure 2.19: (a) Infrared image of specimen WJD1 side 2 without any wearing surface (b) Close up of the 3 x3"x1/16" water-filled delamination in WJD2 [4].

\subsection{Review on Automated Defect Detection}

Numerous experiments have demonstrated that infrared thermography is an effective tool for detection of subsurface flaws in graphite-epoxy composite materials. Some of them were reviewed in Section 2.7. Research has also proved that the growth of defects can be easily monitored during periodic inspections using infrared thermography.

\subsubsection{Automated defect detection approaches}

Maldague [9] noted the advantages of automation in the field of infrared thermography in 1990. It was observed that automated detection of defects/flaws using software or hardware implemented algorithms can be used improve the detection results. Analysis can be performed not only on a single infrared image but also on a series of infrared images taken over a period of time under controlled excitation. Measuring the temporal response of a structure to a thermal excitation is useful as it is difficult to visually analyze a time series of thermal images. Hence analysis algorithms are required to extract the pertinent information from the time series and present it in one or two images and extract defect location from these images automatically. Such a scheme can be applicable when the excitation can be controlled for example in a pulsed phase thermography applications. When the excitation is not controllable such as a field scenario, a single infrared image can be used for automated flaw detection. 


\section{CHAPTER 2. LITERATURE REVIEW}

An automated algorithm for subsurface defect extraction in infrared or in low spatial content images was proposed by Maldague [23]. In this approach potential defects are located in an image by spatial sorting. Then they are placed in a decreasing order based of importance based on their brightness. The location of the hottest pixel for every defect found in the image is calculated by the algorithm. The algorithm assumes that all defects have at least one pixel at a higher value than the image average brightness (AVG) of the image thus removing background pixels from processing. The pixels in consideration are compared to AVG and if found greater, are loaded in a new structure comprising four vectors: POSX [ p ] position along $\mathrm{x}$ in I; POSY [ p ] position along $\mathrm{y}$ in I; INTS [ p ] brightness, that is pixel value I ( $\mathrm{x}, \mathrm{y})$; CLAS [ $\mathrm{p}$ ] class associated to pixel $\mathrm{I}(\mathrm{x}, \mathrm{y})$

Then a sorting procedure was used to sort the INTS[p] array in a decreasing order, followed by processing of the POSX and POSY vectors. This ensures that neighbouring pixels belonging to one class will be close together in the vector structure. The sorted pixels are then labeled using a tolerance value $\mathrm{T}$ found experimentally. The labeling process is restricted to ten classes. The procedure is repeated iteratively with $\mathrm{T}=\mathrm{T}-1$ until same results are obtained from two consecutive iterations. At this stage all the seeds (high temperature points) corresponding to each defects are known.

An iterative procedure is then used for each class to determine the boundary to the potential defect. Pixels neighbouring to the current seed with intensity greater than a threshold 'TH' are agglomerated. The procedure is repeated by decreasing the value of TH until an image boundary is hit. At this point, the vector holds for all the potential thresholds $\mathrm{TH}$, the corresponding number of pixels around the current seed. From this vector of potential defect points the actual ' $\mathrm{TH}$ ' corresponding to the defect area is found experimentally. The process is explained in detail in [23].

This algorithm was implemented on a PC-386, at $16 \mathrm{MHz}$, in $\mathrm{C}$ language. The images have 68 rows by 105 columns (7140 pixels). It takes around $45 \mathrm{~s}$ on the average, restricting the pixels to those above the image average. Many different experiments were run on a variety of different samples with artificial embedded defects. Some of the results 
of defect detection on infrared images of aluminium honeycomb sheets and graphite epoxy panel are shown in Figure 2.20.

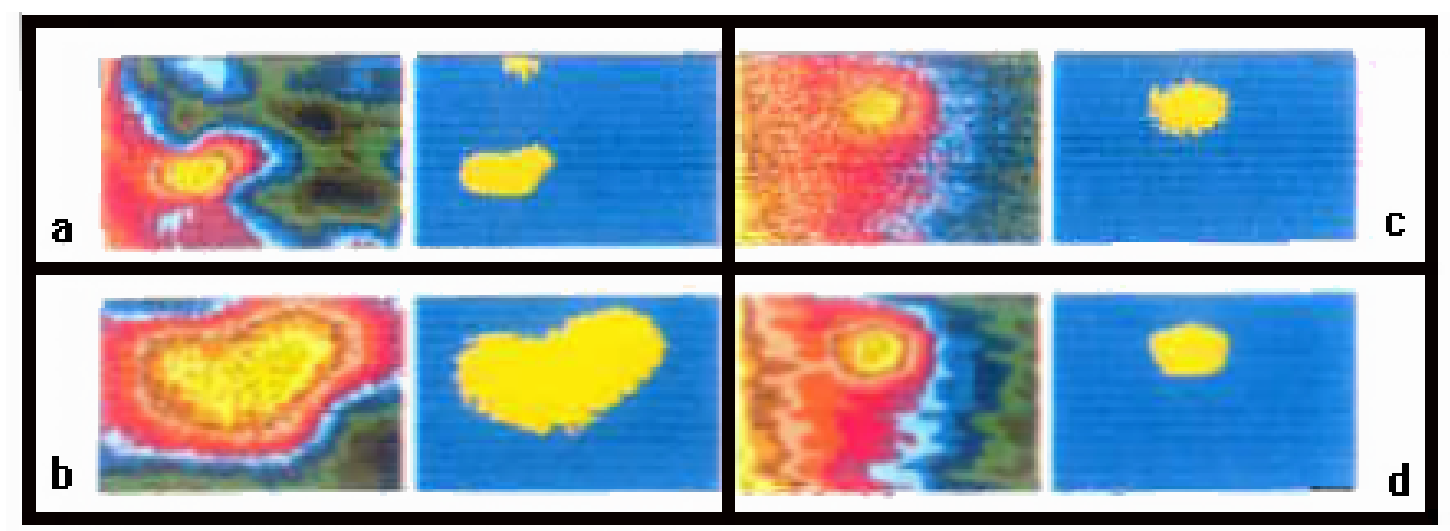

Figure 2.20: (a)(b) Infrared image of aluminium honeycomb with bonding defect and the processing result (c)(d) Infrared image of graphite epoxy panel with artificial defect and processing result [23].

A technique for enhanced defect visualization in composites via transient thermography was presented by Plotnikov [24]. The aim of this technique is to automatically generate a map of the defects located in the observed area of the specimen. Experimental data was collected on composite panels of different thickness with square inclusions and flat bottom holes of different depth and orientation. The time evolution of the thermal response and spatial thermal profiles were analyzed. It was found that defect visualization is difficult in thermal images of carbon fibers. Hence, pulse phase technique was used for improving defect visibility. Also, image processing techniques for noise reduction and automatic defect shape extraction were tested.

The setup involved using two pieces of graphite-epoxy composite polymer for experimentation. One of them was from a thick stitched aircraft wing box structure. Five square flat bottom cavities were fabricated in the backside of the specimen to various depths. Two flash lamps were used as an active heat source. A series of 200 digitized images with 12 bit resolution produced by the infrared camera with a sampling rate of 1 $\mathrm{Hz}$ were transferred into a personal computer for further image processing.

Figure 2.21(a) shows a background thermogram captured prior to heating. The image of size $140 \times 320 \mathrm{~mm}$ covers the region of the panel. Surface temperature appears 


\section{CHAPTER 2. LITERATURE REVIEW}

hotter in the center in the acquired thermogram, even though the surface has no spatial temperature variations. It is seen that the near surface structure of the panel dominates the thermal response immediately after flash. It was found that dominant vertical lines seen in the thermal images for the first several seconds after heating completely mask the presence of subsurface flaws in these images. A thermogram at $7 \mathrm{sec}$ after the flash is shown in Figure 2.21(b). The shallowest defect (2.0 mm below the surface) appears on this image but is not clearly visible.

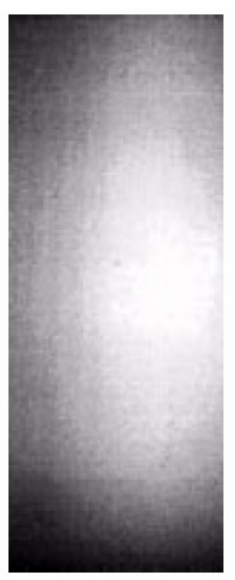

(a)

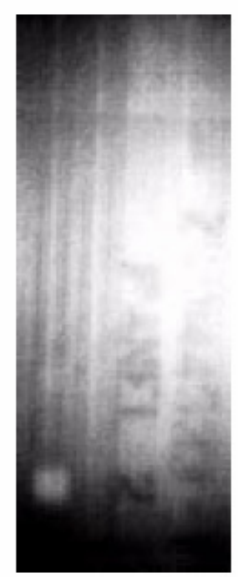

(b)

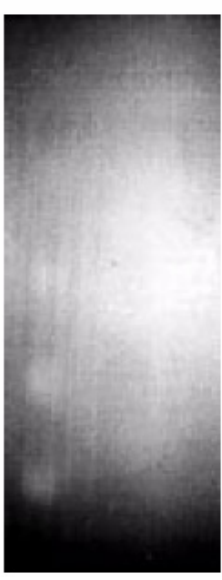

(c)

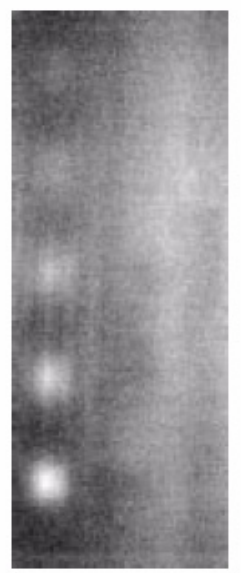

(d)

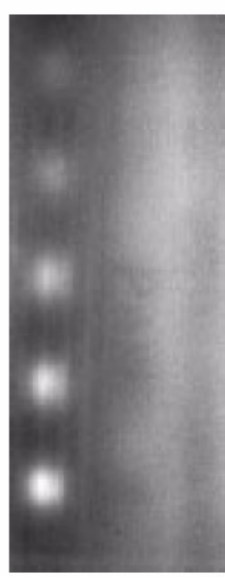

(e)

Figure 2.21: Images of a composite panel with 5 square $(20 \times 20 \mathrm{~mm})$ flat bottom cavities. (a) background image (b) thermal image at $7 \mathrm{~s}$ after the flash (c) thermal response averaged in the interval 10-50 s (d) thermal response averaged in the interval 10-50 s with the subtracted background; (e) phase image [24].

Time averaging of successive thermal images yields, was then used as an image processing technique to improve the visualization of the defects located at different depths. Optimal time interval was determined from the time evolution curves for the points located above the cavities. It was found to be 15 to $45 \mathrm{~s}$ after the application of heat. The thermal response of the surface averaged in the interval 10-50s is presented in Figure 2.21(c). Three square holes are recognized in the image. Background subtraction was then performed using the image in Figure 2.21(a) as the background image. The result is shown in Figure 2.21(d). This image does not contain the bright area in the center any more. All five artificial defects can be localized in this image and a close to normal distribution is obtained in the histogram. 
Finally, Discrete Fourier Transform (DFT) was used to improve the image in Figure 2.21(d). DFT was applied to the temporal thermal response curves extracted from the series of thermograms. The phase of the response at the fundamental frequency was calculated for each pixel using various time intervals. A phasegram shown in Figure 2.21(e) was computed using the above procedure for the time interval 10-100s after heating. The phase image demonstrates a higher sensitivity to the defects and a reduced noise level.

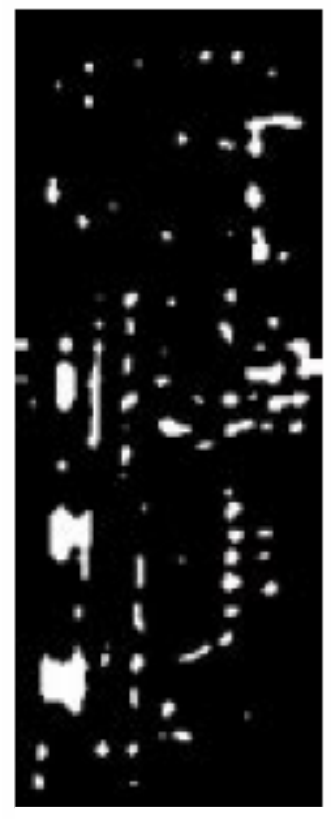

(a)

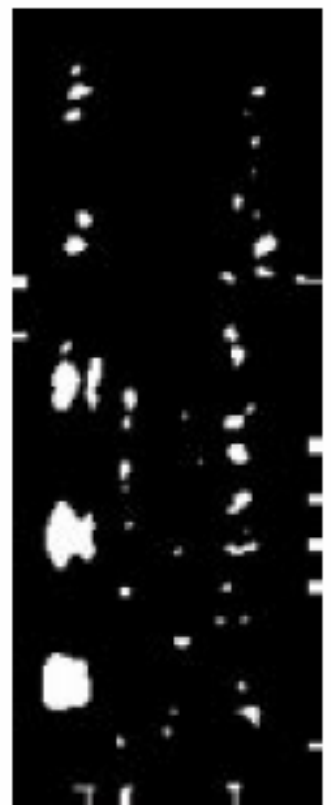

(b)

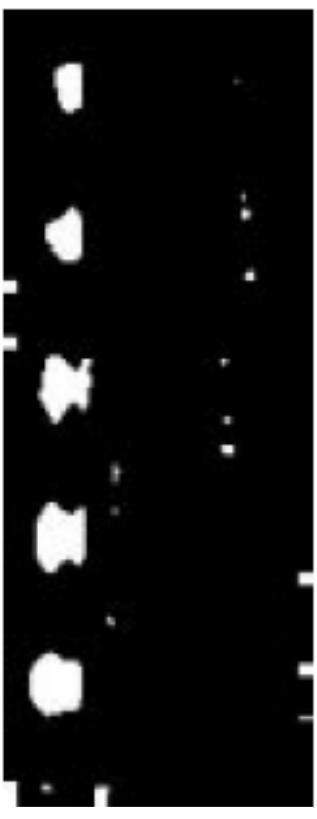

(c)

Figure 2.22: Results of image processing of the (a) Thermograms from Figure 2.21(c) processing result (b) Thermograms from Figure 2.21(c) processing result $\quad$ (c) Phasegram from Figure 2.21 (e) processing result [24].

To obtain a defect map from the images, a laplacian like two-dimensional filter was used for defect size extraction from the images in Figure 2.21(d) and Figure 2.21(e). A $7 \times 7$ element kernel was constructed to give a smoothed approximation to laplacian of an image. A convolution of the kernel and image followed by thresholding is performed to obtain a binary defect map. The defect map in Figure 2.22(a) was obtained from the time-averaged thermal response seen in Figure 2.21(c). It outlines the three defects located 2.0-4.0 $\mathrm{mm}$ under the surface and has the false calls indications caused by the 


\section{CHAPTER 2. LITERATURE REVIEW}

stitches and paint marks on the panel's surface. Figure 2.22(b) shows a defect map computed from the background subtracted thermogram image. It contains fewer false calls. A defect map computed from the phasegram is shown in Figure 2.22(c). It has improved defect images as compared to the averaged thermogram and less false calls.

This algorithm enables defect detection from a thermal image without prior information about the number of defects and their location. The threshold parameter controls the quality of the resulting defect map. An empirical rule for selection of the threshold was developed to enable detection of the maximum number of defects without a significant number of false calls.

One of the main disadvantages of using infrared thermography is said to be the initial cost setting up an automated fault detection system. Infrared cameras themselves are costly to purchase. Recent technological developments however, have helped in manufacturing low cost thermal infrared cameras that are suitable for applications like law enforcement vehicle safety, process control etc. The setup for such a low cost automated fault detection system is explored by Rigney et. al. [28]. The authors used a PalmIR 250 low cost infrared camera with a resolution of 320x240 pixels. The thermal data from the camera was processed by image processing/analysis applications developed using NI LabView and IMAQ Vision Software. In the first phase of testing the researchers adjusted the camera parameters like offset response, temperature sensitivity, non linear step response, gradient response etc. Next they used the camera to capture thermal images of faulty and fault free parts in different applications.

First a system was built for automated inspection of injection moulded rubber parts. Two different injection moulded rubber parts containing deep sprue defects were investigated. A deep sprue defect is characterized by a local depression or absence of material at the part surface. Conductive heating as a means of thermal excitation was achieved by placing the part on a steel plate. A sequence of 26 images, consisting of every fourth video frame over a period of approximately four seconds, was digitized and used for processing.

To analyze the image for defects, a region of interest (ROI) excluding the sprue location was defined within the cup bottom. The average image intensity within the ROI 
was used as a reference for image analysis. A sequence of contrast images was captured by subtracting the reference image intensity from that of each image pixel and normalizing the result by the reference intensity. Then, sequences of contrast slope images were computed by subtracting the time zero contrast image from each subsequent contrast image. The maximum contrast and contrast slope values for each pixel were accumulated over the image sequences to yield the images shown in Figure 2.23(c). The maximum contrast image in Figure 2.23(b) shows that the deep sprue defect was the only region on the part that got warmer than the ROI. Parts without the deep sprue defect yielded images with no or significantly subdued response at the part center. Thus the defect could be identified solely from the maximum contrast image as shown in Figure 2.23(a).

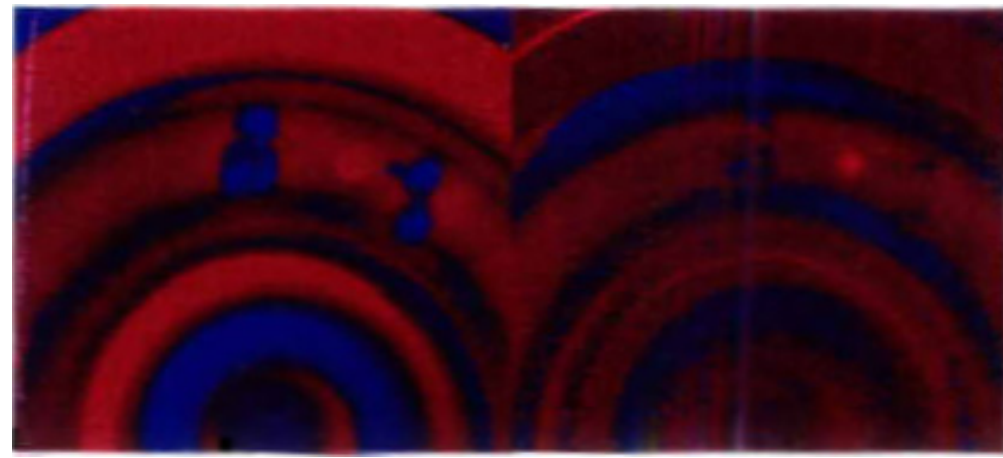

(a) (b)

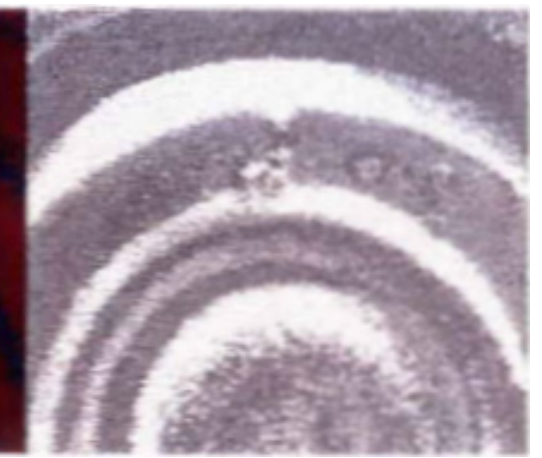

(c)

Figure 2.23: (a) Maximum contrast image for part containing deep sprue defect (b) Maximum slope image for part containing deep sprue defect (c) Image of the defect [28].

The approach was also used by the researchers [28] for the detection of parts with blister defects. In addition to the previously described processing each pixel was fit to an exponential function. The amplitude and exponent of the fitted function were then used detection of the blisters. The researchers have thus shown the use of a low cost infrared camera for development of an automated defect detection system.

Image processing for automated analysis of thermal images is generally done at the post processing stage. Recent developments allow insertion of image analysis capabilities in the infrared camera itself. The need for the use of image processing in 
infrared camera design has been explored by Allred et. al. [29]. The authors argue that the use of focal plane arrays result in high cost infrared cameras. Moreover the presence of a bad sensor in these infrared cameras can make the entire system useless for automated scientific applications. The use of an alternative approach with a hybrid camera design using a rotating multisided mirror and a sensor array is suggested by the author. As the mirror rotates each row of the sensing element will produce a complete image. With this design it can be possible to achieve a high frame rate of 1000 frames per second. Filtering operation can be performed on thermal data acquired at high frame rates.

The authors show that by applying morphological filters to the redundant images, impulse errors can be eliminated if they are not repeated from frame to frame. The use of WMMR (Weighted Mean of Minimum Range) filter for eliminating noise and enhancing the edges is suggested. A modification to the original WMMR scheme to simplify the calculation of WMMR is shown. These filters can be implemented in the infrared camera hardware itself to remove noise and enhance the thermal images.
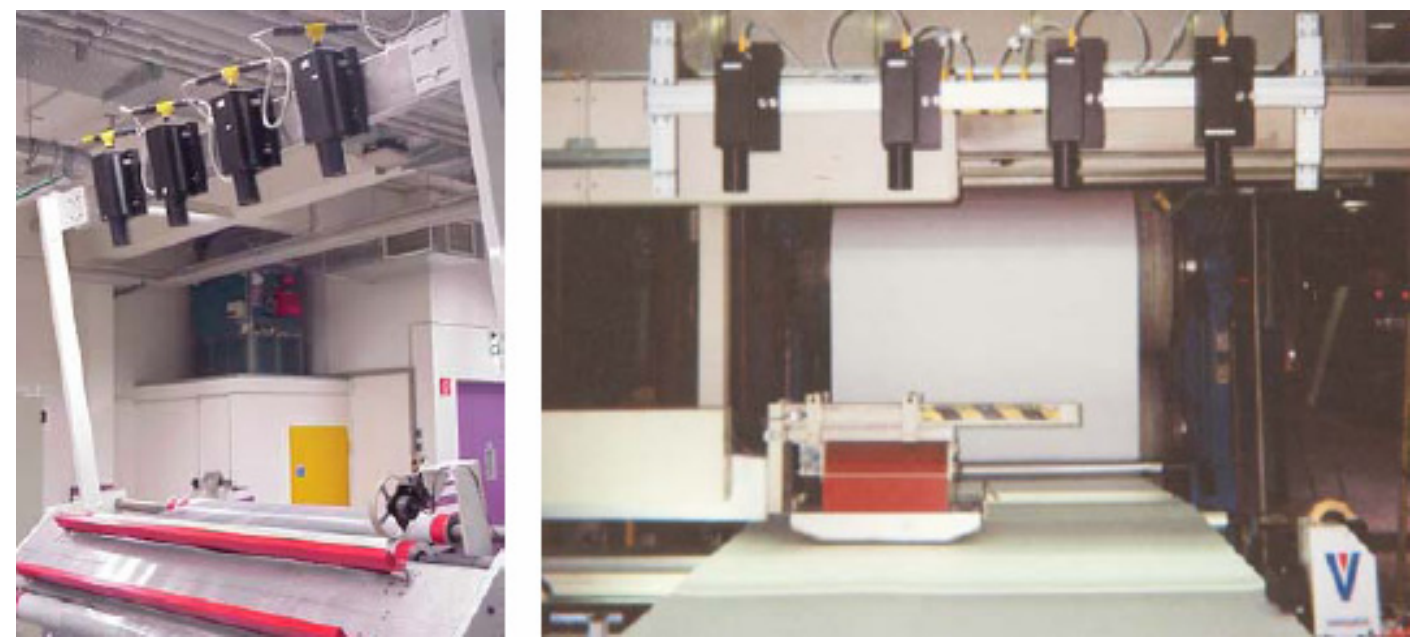

Figure 2.24: On-line inspection system implemented in [30].

The advancements in thermal infrared camera technology as well as increase in data acquisition and processing speed is enabling the development of low cost automated system for analysis of thermal images. Example implementations of such an automated inspection system are found in [30, 34]. The authors in [30] explore the advantages of an 
automated web inspection system over statistical sampling techniques and human inspection. The implementation of an on-line inspection technology is discussed. A detailed description of the system implemented for on-line detection of flaws in manufacture of cast film used for pharmaceutical packaging is given. Figure 2.24 shows the implementation of the on-line inspection system.

The process of manufacture of cast film results in a clear film with frequent carbon specks, gels, gels with carbon specks, and occasional bubbles. The frequency of these defects is controlled by the inspection system. Large catastrophic defects are mapped for converting and smaller defects are trended for process control. Real time alarm limits are set for allowable frequencies of each defect type and size class. When a defect alarm occurs after automated defect detection, an alarm light flashes, and the operator places a flag in the roll and then acknowledges the alarm via a Human Machine Interface (HMI), which changes the alarm light from a flashing status to an illuminated status. Details about different alarm conditions and operator actions are given in [30].

The work in [34] directed towards development of a turn-key test system for IR imaging. The task of creating an automated test for many process control applications is simplified using this setup developed by Santa Barbara Infrared Inc (SBIR). They developed a framework for an automatic test system that could have universal applications using a relatively small fill-in-the-blanks menu set to allow the test engineer to define the specifics for his particular application. The system is designed to work with SBIR's off-the-shelf target projectors shown in Figure 2.25. It provides a turnkey solution for IR imaging testing. With this product, the systems design and integration are already done: the hardware works together, the test algorithms are designed and debugged, and standard reports are printed. A really good user interface with a versatile and easily configurable test set was developed for the test engineer. 


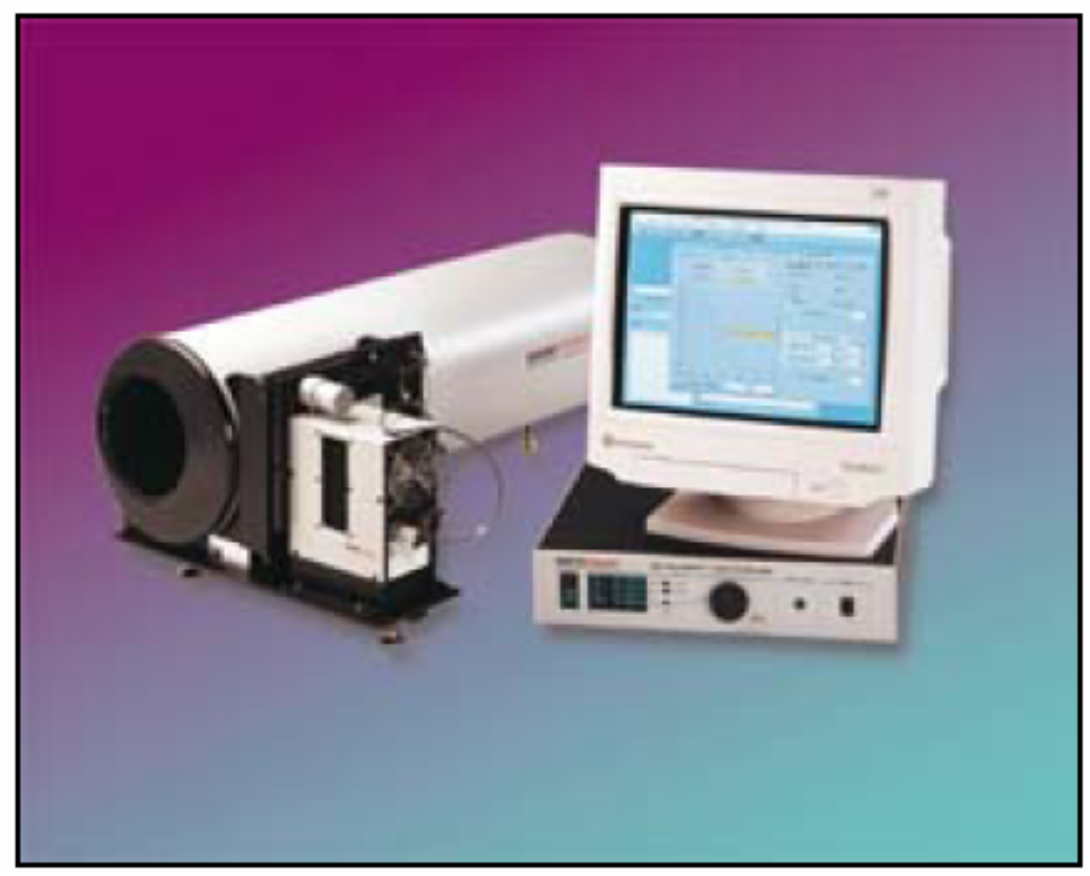

Figure 2.25: SBIR automated IR test system [34].

\subsubsection{Filtering of Thermographic Data}

Infrared images obtained by transient thermography may generate data that is noisy and blurred. The defect identification by an inspector, in some cases is hindered by this spatial noise and/or by the thermal blurring due to lateral diffusion. Reconstruction of the actual thermal pattern from this data presents a challenge that has been addressed by many researchers. The approach by Lhota et. al. [25] involved developing an advanced spatial filtering technique that uses a point spread function based on a previously proposed concept of Thermal Modulation Transfer Function. The proposed approach helps in removing the blurring of infrared images. An enhanced image without the noises that is easier to evaluate was obtained by spatially filtering out the noise frequency components. This was achieved as follows:

$>$ The image was transformed to the spatial frequency regime using a two dimensional Fast Fourier Transform (2D FFT).

$>$ The noise frequency components were identified and amplitudes were reduced.

$>$ The modified 2D FFT data was transformed back to a spatial image with an inverse 2D FFT. 
The resulting image was spatially filtered using a high pass filter (removing the low frequency components). The output image was simpler to evaluate with less noise. Spatial filtering technique was also found to be useful in the evaluation of a sequence of images. An important method of evaluating thermographic data was creating a slope (time derivative) image from an image sequence. In an electronically noisy environment (e.g. number of inductors and transformers in the immediate vicinity), the infrared images have spatial noise that has regular pattern and changing in time. Figure 2.26(a) shows a pulsed thermographic image of a composite part with a diagonal pattern. The noise varies with time and has a repetitive pattern as seen in Figure 2.26(b). The spatial frequency components associated with the diagonal patterns were filtered out of each image of the sequence. The slope image (Figure 2.26(c)) of the filtered image sequence revealed the subsurface details required for evaluation.

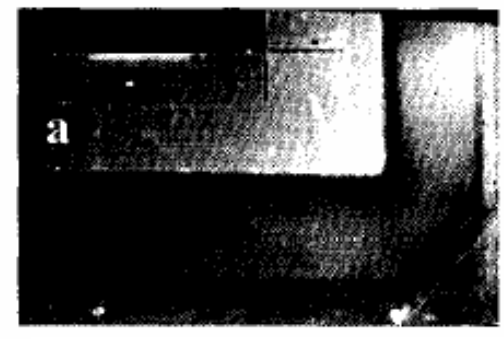

(a)

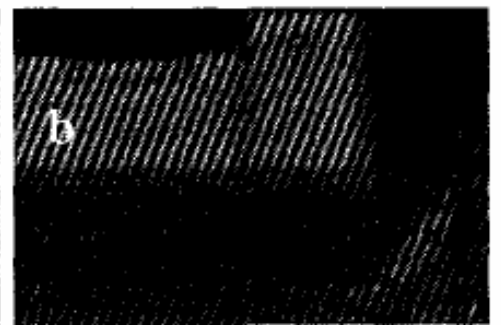

(b)

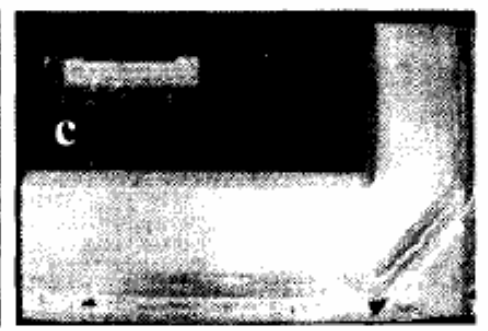

(c)

Figure 2.26: (a) A subtle diagonal noise pattern was present in the image. (b) The pattern was changing in time and dominates the slope image. (c) Image with the desired subsurface details for the inspector's evaluation after spatial filtering [25].

The advanced filtering technique developed in the approach by [25] is an extension of the Thermal Modulation Transfer Function (TMTF) concept introduced by [26]. TMTF allows the determination of critical defect size at a particular depth for a particular sample type. The observed values are compared with the calibration standards generated with programmed defects. The MTF is defined as the Fourier Transform of the point spread function (degree of modulation of the output signal of a particular target expressed as a function of the spatial frequency of the target). The TMTF is based on the relationship between the contrast or modulation and the aspect ratio of the defect 
characterized in terms of the spatial frequency or width of the narrowest dimension of the defect.

In order to evaluate the TMTF technique a calibration sample made of 0.5 " thick steel plate with 8 clusters of target slots milled into the backside of the plate was constructed [25]. The combinations of depths and spatial frequencies of the defects result in 8 distinct aspect ratios ranging from 8 to 1 . The results from a typical flash sequence illustrate that although all eight target clusters were detectable, they were not all resolvable. The ability to resolve an individual bar element diminishes for deeper targets, as the contrast between the target clusters and the background decreases and lateral diffusion obscures the spaces between the target bars. The authors propose a combination of depth and spatial frequency approaches to define a "thermographic MTF".

Finally an approach similar to wiener filtering is explored to recover weakly detected defects. The original shape function $g(x, y)$ can be extracted by taking the Inverse Fourier transform of the expression

$$
G(\alpha, \beta)=\frac{P^{*}(\alpha, \beta)}{|P(\alpha, \beta)|^{2}} I(\alpha, \beta)
$$

where

$\mathrm{G}(\alpha, \beta)=$ recovered image in fourier domain.

$\mathrm{I}(\alpha, \beta)=$ observed image.

$\mathrm{P}(\alpha, \beta)=$ thermal point spread function.

The results of using the above mentioned approach is shown in Figure 2.27.

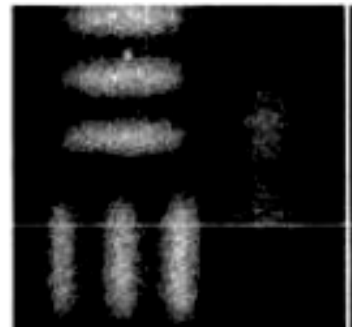

(a)

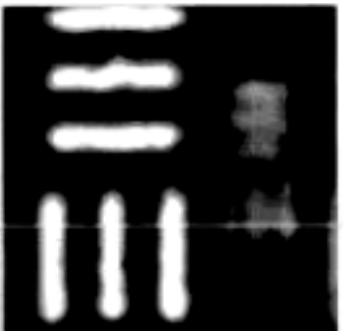

(b)

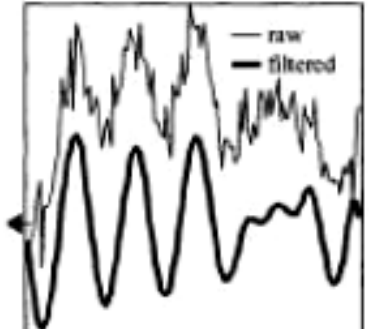

(c)

Figure 2.27: (a) Raw image of resolution target clusters $G$ and $H$ (Left). (b) Improved image using the Thermal Point Spread Function obtained from TMTF analysis. 


\section{CHAPTER 2. LITERATURE REVIEW}

Shepard et. al.[27] have developed a concept of thermographic signal reconstruction (TSR) as a means of enhancing defect to background contrast while reducing the amount of data that must be stored by a magnitude. The technique is useful for obtaining an enhanced image from a time series of pulsed thermographic images. They have shown that a logarithmic representation of the time series data is able to clearly show the shallow and deeper defects. The logarithmic behaviour exhibits remarkable consistency, in that pixels representing defect have a nearly linear time series while the defect pixel time series depart from this pattern. Hence the authors approximate the logarithmic time dependence of a function by:

$$
\operatorname{Ln}(T(t))=a_{0}+a_{1} \ln (t)+a_{2}[\ln (t)]^{2}+a_{3}[\ln (t)]^{3}+a_{4}[\ln (t)]^{4}
$$

The higher order polynomials replicate the noise that appears in the low amplitude data while the low order polynomials serve as an effective high pass filter. The authors then use the approximated time evolution to reconstruct the original signal using the expression:

$$
\frac{d \operatorname{Ln}(T)}{d t}=\sum_{n=0}^{N} n a_{n} \ln (t)^{n-1}
$$

The resulting derivative image offers a higher degree of signal to noise ratio than the time average or background subtracted raw data. In the first derivative image a subsurface feature is observed with amplitude lower than that of background. However, after the background has cooled substantially, the defect cools at a rate faster than the background rate and the amplitude is seen to be higher than that of background.

The derivates created using the TSR approach are observed to be remarkably free of noise, compared to slope images. High sensitivity for shallow as well as deep defects is obtained. An example is shown in Figure 2.28. Figure 2.28(a) shows the logarithmic time plots for background, shallow and deep defect point. The original image and the reconstructed image using the TSR approach is shown in Figure 2.28(b) and (c) 
respectively. It is observed that in the reconstructed $1^{\text {st }}$ derivative image, sensitivity to the deepest holes as well as smaller off center holes is significantly better than in raw images.

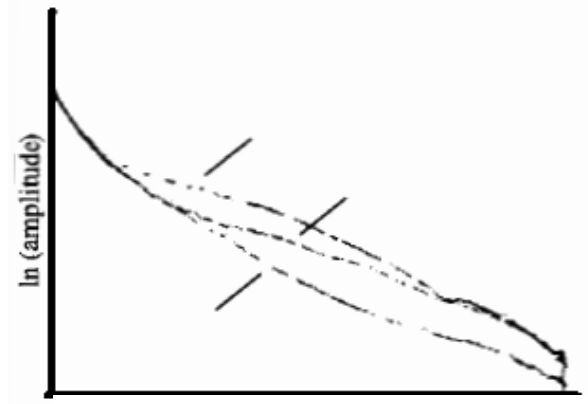

(a)

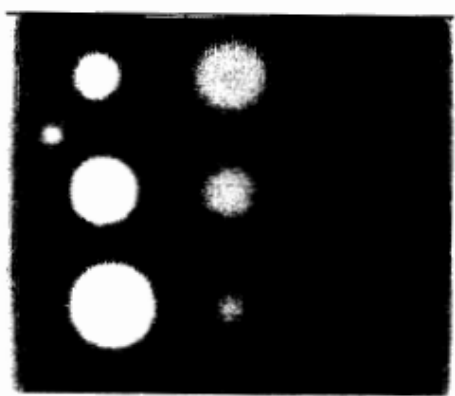

(b)

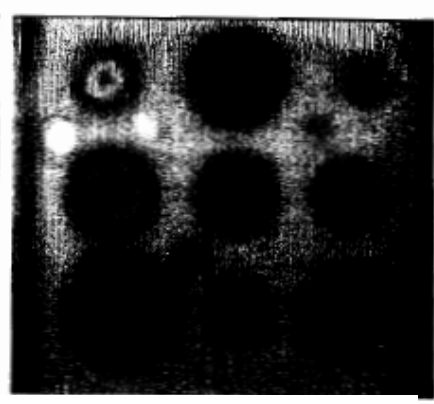

(c)

Figure 2.28: (a) Logarithmic temperature time plots for a background point. (b) raw image data (c) enhanced image using TSR approach. [27]

\subsection{Review on Image Mosaicking}

Registration and mosaicking of images have been in practice since long before the age of digital computers. After the development of airplane technology (1903) aerophotography increased the scope and need of image registration and mosaicking. The limited flying heights of the early airplanes and the need for large photomaps, forced imaging experts to construct mosaic images from overlapping photographs. This was initially done by manually mosaicking images which were acquired by calibrated equipment. The need for mosaicking continued to increase later in history as satellites started sending pictures back to earth. Improvements in computer technology became a natural motivation to develop computational techniques and to solve related problems. There have been a variety of new additions to the classic applications mentioned above that primarily aim to enhance image resolution and field of view. The basic steps for image mosaicking in any application however, are almost the same. They are:

\footnotetext{
$>$ Geometric Correction

$>$ Image Registration

$>$ Eliminate seams
} 
CHAPTER 2. LITERATURE REVIEW

\subsubsection{Geometric Correction}

Geometric correction refers to a transformation that relocates image points. It is also known as image warping. The image warping transformation can be global or local in nature. Some of the most common global transformations are affine, perspective and polynomial transformations. The transformations can be used to represent camera motion. A camera motion can cause a change in scale, rotation, vertical and horizontal shear and altered aspect ratio. These operation change the coordinates of a pixel $\boldsymbol{p}$ located at position $(\mathrm{x}, \mathrm{y})$ to a new location $\boldsymbol{p}$ '. The new location can be calculated using the expression:

$$
p^{\prime}=A p+t
$$

where $\mathbf{t}$ is the translation component and $\mathbf{A}$ controls scaling, rotation and shearing parameters. A can be of the form [50]:

$$
\begin{aligned}
& A=s\left(\begin{array}{rr}
\cos (\theta) & -\sin (\theta) \\
\sin (\theta) & \cos (\theta)
\end{array}\right) \quad \text { : scale and rotation } \\
& A=\left(\begin{array}{ll}
1 & 0 \\
a & 1
\end{array}\right) \quad \text { : vertical shear } \\
& A=\left(\begin{array}{ll}
1 & \mathrm{a} \\
0 & 1
\end{array}\right) \quad \text { : horizontal shear } \\
& A=\left(\begin{array}{ll}
S_{x} & 0 \\
0 & \mathrm{Sy}
\end{array}\right) \quad \text { : altered aspect ratio }
\end{aligned}
$$

The affine transformation can be represented by a single matrix multiplication in homogenous coordinates:

$$
\left(\begin{array}{l}
X^{\prime} \\
Y^{\prime} \\
1
\end{array}\right)=\left(\begin{array}{ccc}
a_{11} & \mathrm{a}_{12} & \mathrm{a}_{13} \\
\mathrm{a}_{21} & \mathrm{a}_{22} & \mathrm{a}_{23} \\
0 & 0 & 1
\end{array}\right)\left(\begin{array}{l}
x \\
y \\
1
\end{array}\right)
$$

By introducing a scaling parameter $\mathrm{W}$, the transformation matrix $\mathrm{A}$ can be modified to handle perspective corrections: 


$$
\left(\begin{array}{l}
X^{\prime} \\
Y^{\prime} \\
W
\end{array}\right)=\left(\begin{array}{ccc}
a_{11} & \mathrm{a}_{12} & \mathrm{a}_{13} \\
\mathrm{a}_{21} & \mathrm{a}_{22} & \mathrm{a}_{23} \\
\mathrm{a}_{31} & \mathrm{a}_{32} & 1
\end{array}\right)\left(\begin{array}{l}
x \\
y \\
1
\end{array}\right)
$$

New image coordinates can be found as $x^{\prime}=X^{\prime} / W, y^{\prime}=Y^{\prime} / W$. Alternatively, perspective transformations are often represented by an 8 parameter model known as homographies. The 8-parameter homography accurately models a perspective transformation between different views for the case of a camera rotating around a nodal point.

\subsubsection{Image registration}

Registration is the central task of image mosaicking procedures. One of the simplest means of image registration is manual alignment. However, they are being increasingly replaced by automated image registration methods that can be categorized as [36]:

1. Correlation Methods: Cross correlation is the basic statistical approach to registration. It gives the degree of similarity between the image and a template. If the template matches the image exactly except for an intensity scale factor and a translation $(i, j)$ the cross correlation will have its peak at $\mathrm{C}(i, j)$.

2. Fourier Methods: Frequency domain approaches for finding displacement and rotation/scale are computationally efficient but can be sensitive to noise. These methods also require the overlap extent to occupy a significant portion of the images

3. Point Mapping: This type of approach is used for two images whose type of misalignment is not known. It consists of finding feature points or control points in the two images to be aligned. The control points are compared to find the best possible match for an affine, perspective or a polynomial transform. The nonreference image is then resampled to a new image using the calculated transformation. 
4. Elastic Model Based Matching: In this method, instead of directly applying piecewise interpolation on a transformation computed from a set of control points, the distortion in the image is modeled as an elastic distortion. The image object is modeled as an elastic body and similarity between points or features in the two images act as external forces which stretch the body.

Some of the literature on image mosaicking relevant to the mosaicking of infrared images to form a "composite image" is reviewed in this section. Infrared imaging has found many applications, some of which are discussed in Section 2.6. Many geo-satellite and aerial applications involve capturing both a thermal image as well as a visual image of the field of view. The registration of thermal images with images from other sensors has been explored by many researchers. Infrared image data is also fused with other image data such as Synthetic Aperture Radar (SAR) images etc. Registering images from two different modalities is a challenging task as the images generated by the two may have different features. The work by Hines et. al. [37] discusses the development of an Enhanced Vision System (EVS) utilizing multi-sensor image fusion currently under development at the NASA Langley Research Center. Multi-spectral images obtained from a short wave infrared (SWIR), a long wave infrared (LWIR), and a color visible band CCD camera, are enhanced and fused using the developed algorithms. The images from these different sensors do not have a uniform data structure: the three sensors not only operate at different wavelengths, but they also have different spatial resolutions, optical fields of view (FOV) and bore-sighting inaccuracies. Thus in order to perform image fusion, the images must first be co-registered. The authors present two methods for registering the infrared and CCD images.

The first algorithm proposed by the authors is based on simply correcting camera sensor specification differences through cropping, scaling and image resampling, assuming that the cameras are bore-sighted and hence share a common center. The images from the three different sensors are cropped so that all three cover the same area. The LWIR image is then resized to the size of the SWIR image. A similar piece of operation is performed between the SWIR image and the visible image. Tests based upon 


\section{CHAPTER 2. LITERATURE REVIEW}

this method of registration showed that the assumption that the cameras were perfectly bore-sighted was invalid and adjustments had to be made to correct for the differences in sensor orientation and alignment.

The second algorithm was a more general, geometric image to image registration algorithm. The distortions between the images were observed to be because of sensor translation, (slight) rotation, scale change and possibly shear. An affine transform was thus used to model the spatial transformation. Control points were manually selected for identifying and matching corresponding features between the reference and distorted images. Since the sensors are assumed to have the same alignment over time, only one baseline set of images need to be registered. The same transformation can subsequently be used for the rest of the image frames. The defined affine transform provides a mapping between the baseline and distorted images. The distorted image is then resampled using the transform parameters to create the registered image. The results of registering the SWIR, LWIR image with the visible image are shown in Figure 2.29.

A real time implementation of infrared and SAR data fusion algorithm is carried out by $\mathrm{Li}$ and Zhou [38]. A new approach to multi-sensor fusion at the pixel level is explored. The feature extraction and target detection are all carried out in the fused image. This method helps in increasing the accuracy of the subsequent operations and increase in the overall performance of the ATR system. The system contains three major components: preprocessing, registration and fusion.

The authors use averaging of neighboring pixels or averaging multi-channel pixels to reduce noise in the image. The multi-channel averaging found to be a better technique more suitable for multi-channel data like SAR images. The averaging was followed by a median filtering procedure for image enhancement. The next step was image registration to establish the correspondence between two images and determine a geometric transform that aligns one with the other. An automatic feature based registration algorithm was used. 


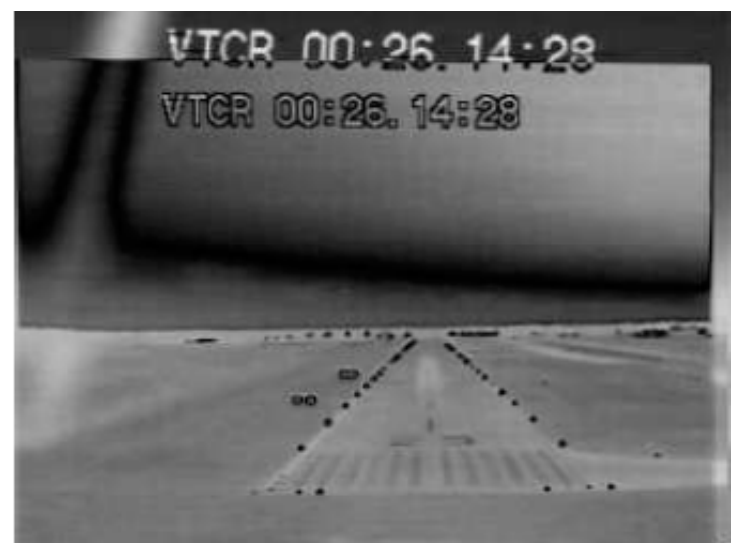

(a)

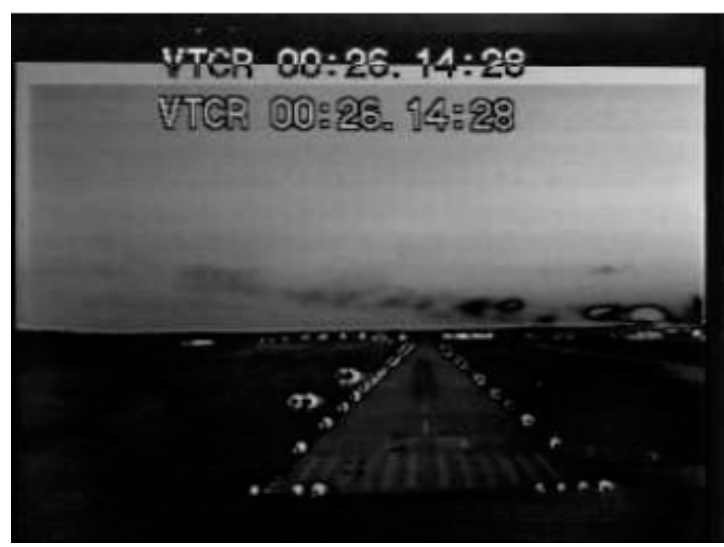

(b)

Figure 2.29:(a) LWIR image registered with visible image (b) SWIR image registered with visible image [37].

It uses point features as control points in aligning two images. The point features referred to as "consistent points", are extracted from the image intensity directly by a Gabor feature extractor. To identify consistent points, a multi-stage point matching scheme is used. The method consists of four steps: (1) Point feature extraction; (2) Point feature matching; (3) Consistency checking; and (4) Image transformation. The output of the image transformation is used to register the two images together. Finally, image fusion is carried out to combine the multi-sensor images into a single image that is more suitable for visual perception, computerized object detection and target recognition etc. More details about the image fusion scheme are listed in [38]. Figure 2.30 shows the results of SAR/IR image registration and fusion using the above mentioned approach.

Many geo-satellite applications involve joining IR images to form a mosaic that gives an aerial view of the entire area in consideration. Mosaicking has also gained importance in other areas such as joining deep sea image to form a complete view of the sea bed. A review and implementation of some of the successful techniques in mosaicking for underwater imaging was done by Eustics et. al. [39]. The authors developed an extended image processing toolbox that implements some useful optical image processing and image mosaicking algorithms found in literature. The algorithms implemented include contrast limited adaptive histogram specification (CLAHS), fourier based methods, cross-correlation based methods, multi resolution based methods etc. The 
algorithms were implemented to explicitly deal with the unique constraints of underwater imagery to build a mosaic of the deep sea image.

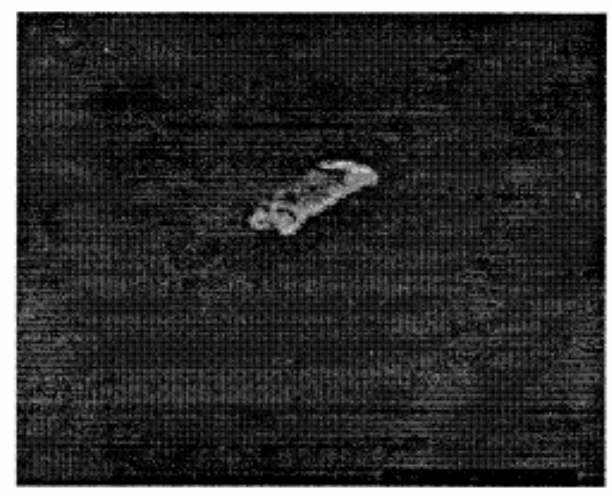

(a)

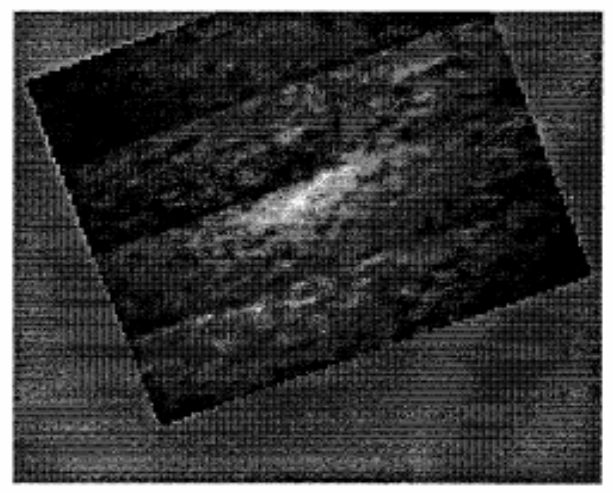

(d)

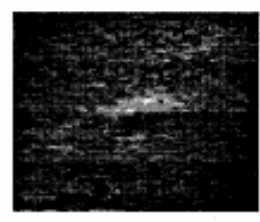

(b)

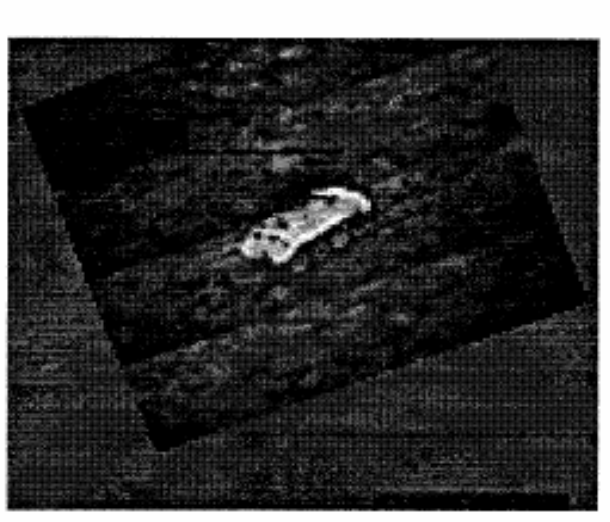

(e)

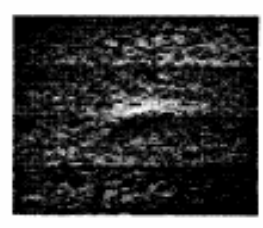

(c)

Figure 2.30: (a) The original IR image. (b) The original even polarized SAR image. (c) The original odd polarized SAR image. (d) The smoothed SAR image registered with the IR image. (e) The fused image [38].

The presence of lighting artifacts in the image due to uneven lighting increases the difficulty in image registration. To compensate for this CLAHS was employed as a preprocessing step. The idea behind this technique is to first divide the original image into equal area contextual regions. Each region is then histogram equalized whereby a monotonically decreasing grey level transform is determined for each region which maps the local grey level histogram to an ideal grey level distribution. The overall effect is to limit the slope of the cumulative histogram which is used in the calculation of the grey level transform. The authors have concluded after experimentation that the Rayleigh 


\section{CHAPTER 2. LITERATURE REVIEW}

distribution is most suited to underwater imagery. Next a fourier based registration method for finding registration parameters is implemented. The phase shifting property of the fourier transform is exploited. The translation between two images is estimated by locating the impulse associated with the inverse transform of the cross power spectrum of the two images using the equation:

$$
\begin{aligned}
\frac{F_{2}\left(w_{1}, w_{2}\right) \bullet F_{1}\left(w_{1}, w_{2}\right) *}{\left\|F_{2}\left(w_{1}, w_{2}\right) \bullet F_{1}\left(w_{1}, w_{2}\right) *\right\|} & =e^{-j\left(w_{1} x_{0}+w_{2} y_{0}\right)} \\
& =\delta(x-x 0, y-y 0)
\end{aligned}
$$

Modifications to the above expression are used to determine the rotation and scaling factors. Finally since the set of translation, rotation and scaling parameters obtained by the fourier based method may not be sufficient to obtain perfect registration a local normalized correlation method is used. Local normalized correlation surfaces are calculated for each pixel to determine a dense set of correspondence between images. This dense set of correspondences is then pruned by only considering pixels which have a concave correlation surface as a reliable match. The mosaic obtained after the above process is shown in Figure 2.31.

The image in Figure 2.31 clearly shows the edges of the mosaicked regions. There is a discontinuity between any two images in the mosaic. Once the mosaic is generated, a technical problem in image representation is joining image borders so that the edge between them is not visible. Hence, a multiresolution pyramidal blending approach is used. Here, the two images are decomposed into different band-pass frequency components merged on those levels, and then reassembled into a single seamless composite image. The idea is that with this technique the transition zone between bandpass image components can be appropriately chosen to match the scale of features in that band-pass component. A more detailed explanation of multiresolution pyramidal blending is given by Burt [40]. The result of blending two images in a mosaic to remove the edge discontinuity is shown in Figure 2.32. 


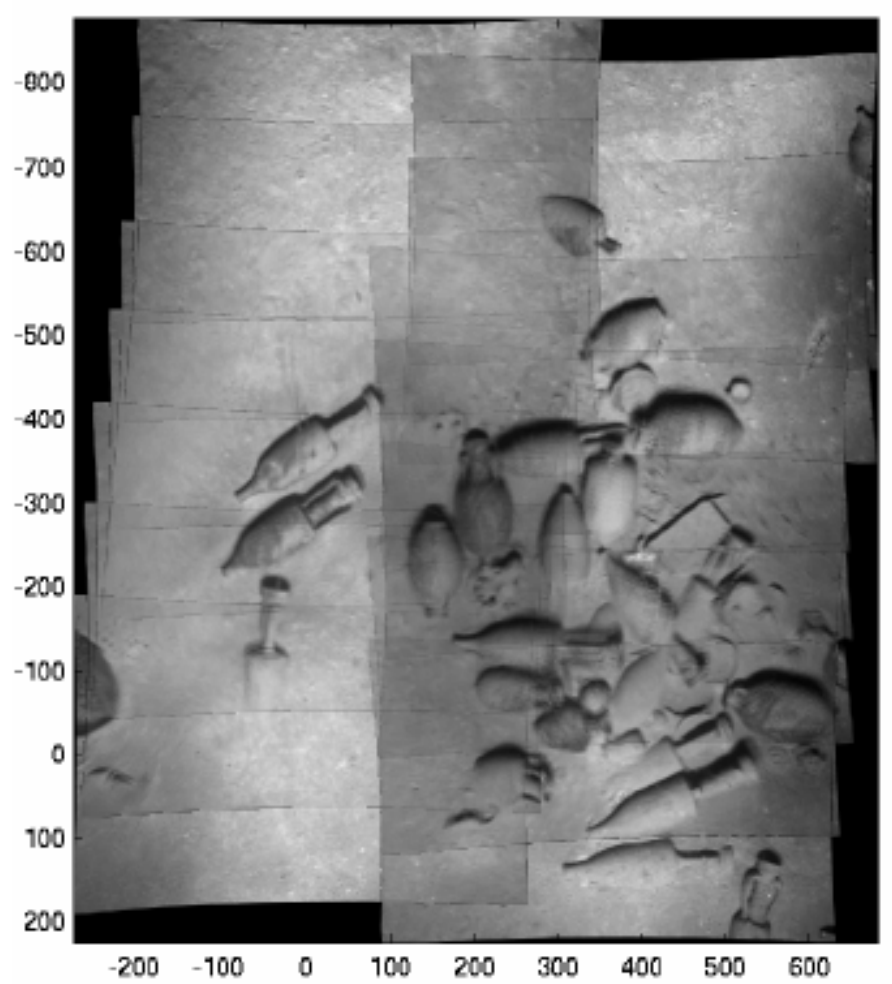

Figure 2.31: A sequence of images mosaicked together utilizing all available cross linked image correspondences [39].

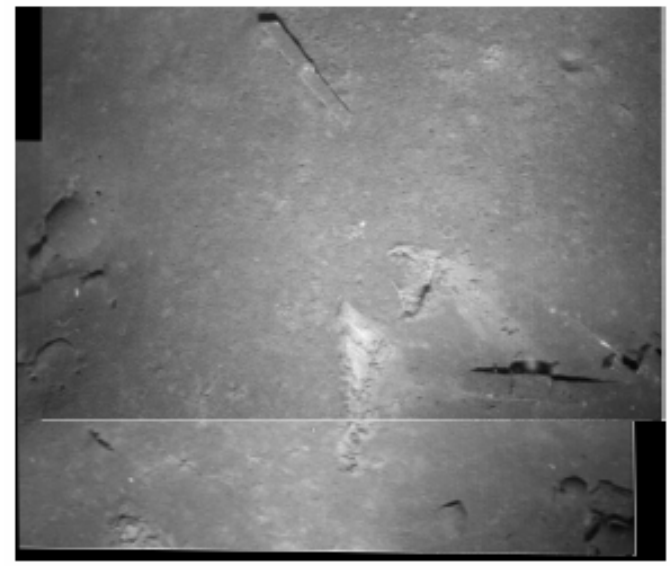

(a)

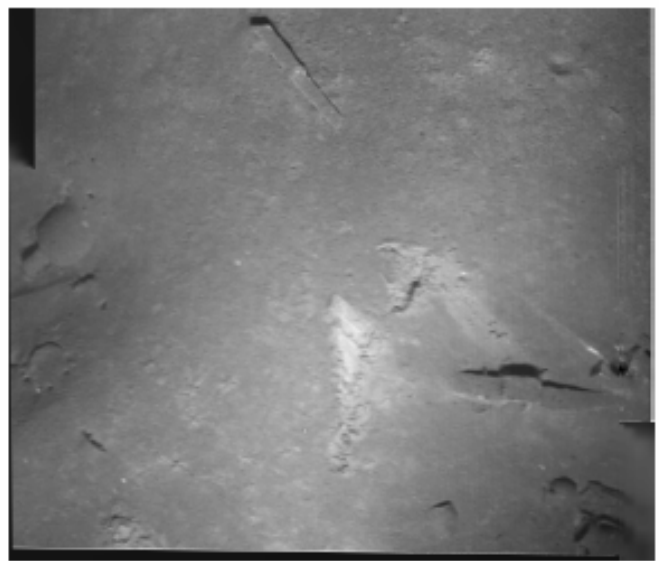

(b)

Figure 2.32: (a) A two image mosaic with seam (b) Removal of the seam after blending [39].

Recent developments in hardware processing capabilities have enabled hardware implementation of popular mosaicking algorithms. These algorithms either calculate the registration parameters automatically or through some sensors that give a feedback about camera motion. This reduces computational complexity enabling real time 


\section{CHAPTER 2. LITERATURE REVIEW}

implementation. The camera movement may lead to images that are captured from multiple perspectives. The mosaicking of such images is known as multiperspective mosaicking. An approach for multiperspective mosaicking for inspection of the underside of vehicles for dangerous materials is implemented by Koschan et. al. [41]. The authors use a camera mounted on a mobile platform to develop an automated system for this task. The platform moves under the vehicle that is to be checked while the camera captures video, which can then be used for visual inspection. The aim for their work stems from the need to create high-resolution images by mosaicking a series of infrared and color video data acquired for under vehicle inspection.

The authors placed certain restrictions on the movement of the mobile platform. Firstly, it was assumed that the camera is translated solely on a single plane that is parallel to the plane of the scene. It was also assumed that the viewing plane of the camera is parallel to this plane of the scene. The final assumption is that the camera does not rotate about its principal axis. The movement of the camera is explained in Figure 2.33 .

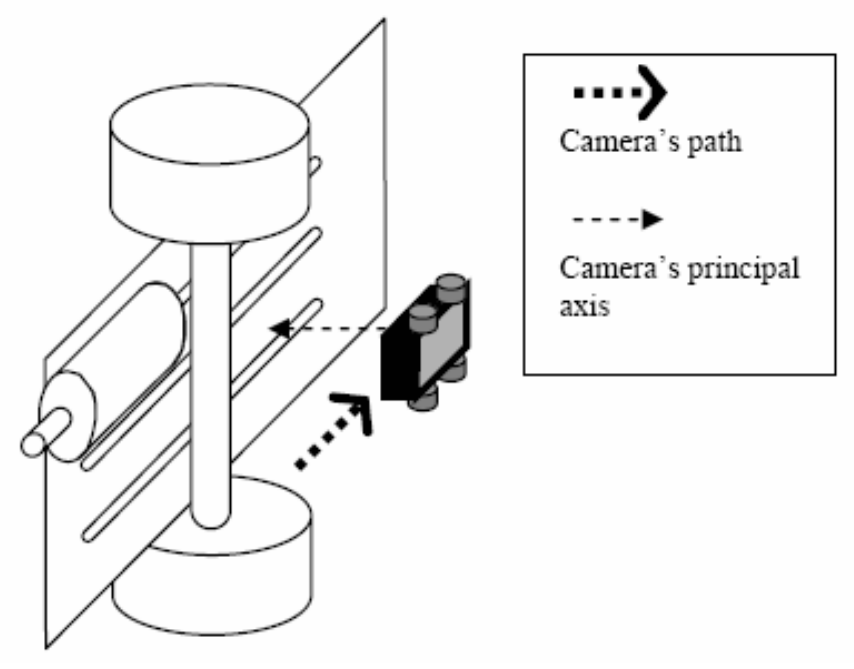

Figure 2.33: Video acquisition setup using a camera mounted on a mobile platform [41].

The process of infrared image mosaicking in [41] can be subdivided into three steps; (1) preprocessing (2) registration and (3) merging to generate a mosaic. Preprocessing stage involves correcting each image in the sequence for barrel distortion 


\section{CHAPTER 2. LITERATURE REVIEW}

and perspective distortion. Next, the motion associated with each frame in the sequence is computed. Motion vector is computed relative to the next frame the sequence. The motion vector $(\mathrm{u}, \mathrm{v})$ may consist of shifts in the horizontal $(\mathrm{u})$ and vertical $(\mathrm{v})$ directions. Dominant motion is computed by the phase correlation method since this technique is capable of extracting dominant inter-frame translation even in the presence of many smaller translations.

Once the horizontal and vertical motions between two images have been computed using phase correlation, strips are acquired from one of the images based on those motions. One of the motions will correspond to the direction in which the camera is moved during acquisition. This is called the primary motion. The other motion which may be due to the camera deviating from a straight path, or the camera's tilt will be orthogonal to the primary motion and is called the secondary motion. The width of the strips is directly related to the primary motion. Adjacent strips on the mosaic are aligned using the secondary motion. Finally a simple blending scheme was used to reduce the visual discontinuity caused by seams.

The process is then repeated for each subsequent frame in the video sequence. After each cycle of the merging process, the vertical and horizontal displacement of the last strip in the mosaic is recorded and this information is used as the anchor for the next strip in the mosaic. Once every frame in the video sequence has been processed, the mosaic is complete. The results for mosaicking with and without blending for the visual as well as thermal images are shown in Figure 2.34. The results of mosaicking a sequence of infrared images from the car inspection are shown in Figure 2.35.
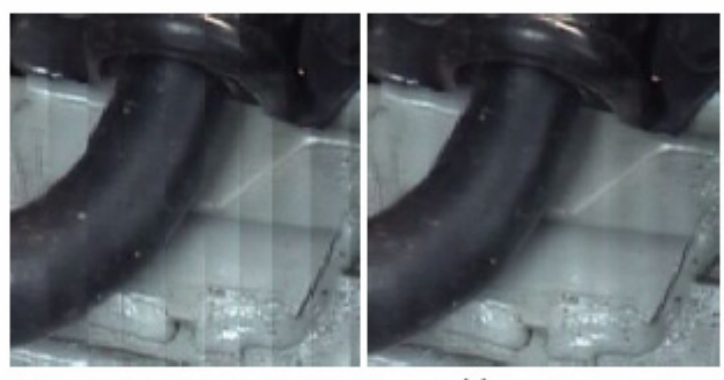

(a)

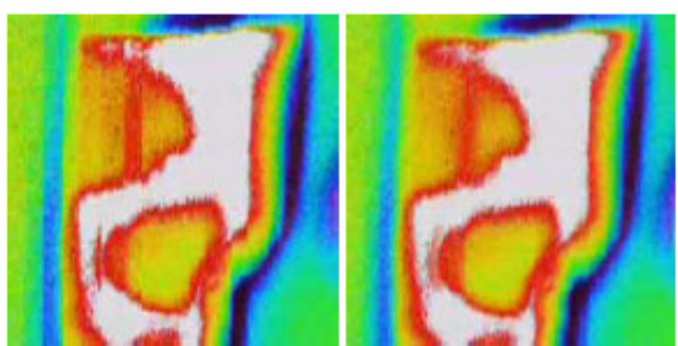

(b)

Figure 2.34: (a) Mosaicking without and with blending for the visual sequence. (b) Mosaicking without and with blending for the thermal sequence [41]. 


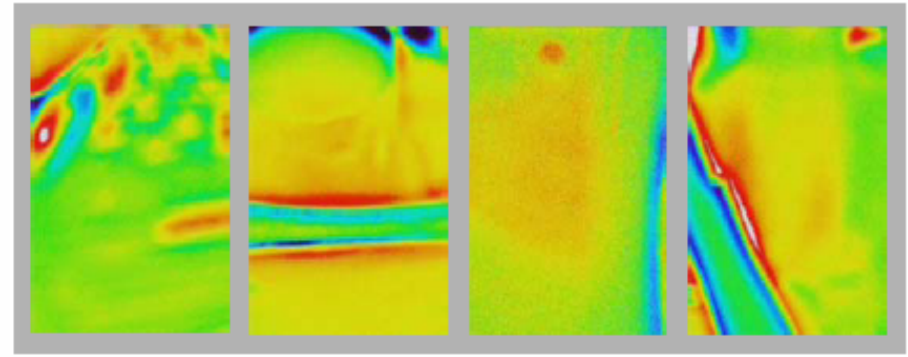

(a)

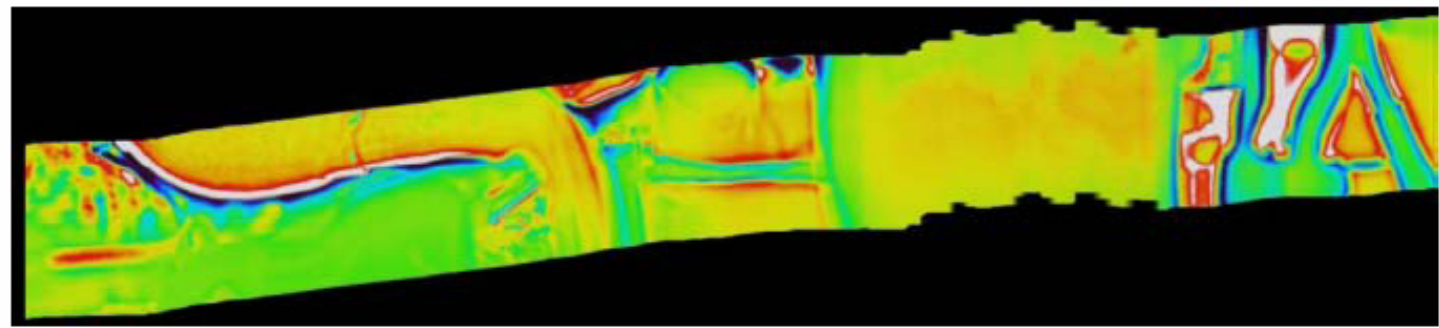

(b)

Figure 2.35: (a) Sample frames from infrared sequence. (b) mosaic generated from the infrared sequence [41].

\subsection{Summary}

Infrared thermography has numerous applications from plant and process monitoring to applications in security and predictive maintenance. The vast amount of data (for e.g. geo-satellite images), varied image feature set and sustained accuracy and reliability needs have increased the necessity for automation in the field of infrared thermography. Many researchers have addressed this need, especially in the fields of process control, condition monitoring, security applications etc. One of the factors increasing the ease of automation in these areas is that these applications are generally in a closed and controlled environment. Hence effects of external disturbances such as heating because of solar radiation, wind etc. are minimized. This helps in designing a system with high accuracy and reliability.

There has been comparatively little effort to automate defect detection in civil engineering infrastructures. However, recent trends have shown an increasing interest in automating many applications (including civil engineering applications) in infrared imaging by using techniques like multi modal image analysis and image mosaicking. 


\section{Chapter 3}

\section{Automated Defect Detection System}

\subsection{Need for Automation}

The analysis of infrared images by a human operator often makes the defect detection process ambiguous. Different human operators can arrive at different defect detection results for the same thermal image. This ambiguity can be minimized using software automated image processing algorithms to detect defects in the thermal image. The need for automation in the field of infrared thermography was noted as early as 1990 by Maldague [9]. Automated testing is the better way to test infrared imaging systems. It eliminates operator error, removes reliance on human perception, and generates repeatable and accurate test results [34]. Also, automated algorithms [2] provide a cost efficient way to process large volumes of data.

Automated testing is practical for the development lab, the end user, or other applications such as periodic condition monitoring systems that generate a large amount of data over a time period. The process of automation is complete with the development of a turn-key system. The turn-key system interfaces the infrared camera with a laptop or a computer. The device records the images captured by the infrared camera and performs analysis to detect anomalies in the infrared images. The anomaly/defects can be displayed in terms of a binary defect map and reports can be generated automatically. 


\section{CHAPTER 3. AUTOMATED DEFECT DETECTION SYSTEM}

\subsection{Problem Description}

FRP bridge decks are being increasingly used for the repair and construction of civil engineering structures such as bridges. Information regarding the long term performance of FRP is not readily available. Also, FRP bridge decks may develop cracks and defects such as delaminations and debonds during its life of operation. It is necessary to detect these deformities as early as possible as they harm structural integrity and may even lead to failure of the deck. Hence periodic monitoring of such structures is necessary.

Infrared thermography provides a non-invasive and efficient method for gathering and storing large amounts of data about subsurface defects with ease. The analysis of such data by human operators is currently in practice. However, human intervention can cause an ambiguity and inconsistency in the analysis of infrared images. Moreover as the size of data increases with periodic monitoring, it becomes cumbersome to track the progress of defects for a certain area in a bridge deck, manually. Automated analysis provides a convenient method to address this issue and improve defect detection accuracy. Care needs to be taken to reduce the false positives (a non-defect area being considered as a defect area) that can occur in such an automated system. The false positives will add an unnecessary cost to the repair of bridge decks. At the same time the accuracy of defect detection should be high, 100\% in ideal conditions (i.e. actual subsurface defects should be correctly identified by the automated defect detection system). An early detection of defects can minimize costly repair.. This constraint makes automated defect detection a challenging task.

Another issue at hand is to correlate the exact position of the defect in an infrared image to its physical location on the bridge deck. A single infrared image does not cover the entire bridge deck area. The ability of infrared thermography to detect minute defects will be adversely affected if the area covered by the images increases, or the distance between the camera and the bridge deck increases. This poses a restriction on the amount of area that can be covered by an infrared thermography in a single image. Though a single infrared image captured in such a manner may be able to detect an anomaly, the exact location of the defect may be difficult to estimate. For this purpose it is necessary to 


\section{CHAPTER 3. AUTOMATED DEFECT DETECTION SYSTEM}

join the infrared images of various parts of the same bridge deck into a composite image mosaic.

This research addresses two main issues mentioned above; i.e. reliable automated defect detection and building a mosaic image from a series of infrared images. A turn-key was built during this research to resolve the issues mentioned above. A turn-key system should be able to: 1) Automate the infrared image acquisition process. 2) Automatically analyze the infrared image for defects. 3) Build an image mosaic from a sequence of infrared images. The details of the development of the turn-key system, algorithms developed for automated defect detection and infrared image mosaicking are explained in this chapter.

\subsection{Development of the turn-key System}

The aim was to develop a prototype turn-key system for automated detection of defects. In the standard automated defect detection setup, images are obtained using the ThermaCAM Researcher software provided by FLIR and are stored on the computer. Then, using one of the tools from the ThermaCAM Researcher software the images are converted from a sequence file into a JPEG image format and stored to a different folder. The automatic defect detection code written in Matlab is finally used to analyze these jpeg images for defects.

This process is tedious and involves human intervention for obtaining and pre processing/converting the input data. Instead, the aim is to automate the entire data collection and analysis process such that it is possible to grab images from the camera and analyze them then and there. This will lead to the development of a turn-key system that is able to interface with the FLIR ThermaCAM S60 infrared camera and automatically capture and analyze defects in infrared images of FRP bridge decks with a single GUI interface. Two methods are tested to develop a turn-key system. They are explained below. 


\subsubsection{AutoIT Scripting Language Method}

AutoIT is a simple tool that can simulate keystrokes, mouse movements and window commands in order to automate any windows based task (or even windowed DOS tasks) [53]. When AutoIT is executed, it reads a specified script file. This script file allows AutoIT to perform a number of functions, these include:

$>$ Executing programs (Windows applications, DOS commands, etc.)

$>$ Sending keystrokes and mouse clicks (all characters, keyboard layout independent).

$>$ Window functions (e.g. minimizing, hiding, restoring, waiting for, activating).

$>$ Simple text clipboard functions.

$>$ Simple registry functions.

> Simple string and variable functions.

An example script is given below

; AutoIt Version: 3.0

; Language: English

; Platform: Win9x/NT

;

; Script Function:

; Prompt the user to run the script - use a Yes/No prompt (4 - see help file)

\$answer $=\operatorname{MsgBox}(4$, "AutoIt Example ", "This script will run the ThermaCAM Researcher to see if the process can be automated. Run?")

; Check the user's answer to the prompt (see the help file for MsgBox return values)

; If "No" was clicked (7) then exit the script

If $\$$ answer $=7$ Then

$\operatorname{MsgBox}(0$, "AutoIt", "OK. Bye!")

Exit

EndIf

; Run ThermaCAM Researcher 
CHAPTER 3. AUTOMATED DEFECT DETECTION SYSTEM

Run("research.exe","C:\\Program Files \\ThermaCAM Researcher 2002", @SW_MAXIMIZE)

;Run, C:\\Program Files \ThermaCAM Researcher 2002\\Research.exe

\$var=@WorkingDir

MsgBox(4096, "Serial Number: ", \$var)

; Finished!

The script above will ask the user if he wants to run the ThermaCAM Researcher tool provided by FLIR. If the user clicks "Yes" the script runs the specified exe file. ThermaCAM Researcher is the tool provided by FLIR to interface with the infrared camera, capture images and perform analysis [33]. An extension of this script can be written that will operate as follows:

1. Open Matlab

2. Open ThermaCAM Research

3. Capture infrared images by clicking on the right menus in ThermaCAM Researcher

4. Save the images as jpeg files

5. Read them in Matlab

6. Run the Matlab automatic defect detection script.

Though such a script may be able to run ThermaCAM Researcher, Matlab and automate the process, the outcome will be a cumbersome solution. ThermaCAM Research and Matlab will need to be installed on the computer for the script will work. Variables such as file names, place of file storage will be fixed in such a scenario. Hence the method was not considered as the best possible way of developing a turn-key solution.

\subsubsection{The FLIR SDK Method}

The second method involved using the ThermaCAM Software Development Kit (SDK) available from FLIR [54], the manufacturers of the infrared camera itself. The 


\section{CHAPTER 3. AUTOMATED DEFECT DETECTION SYSTEM}

SDK provided us with routines that can be used to talk with the camera and automate most of its features such as image acquisition, automatically adjusting the infrared image and so on. The SDK routines can be programmed from the Visual Basic, Visual C++ or the Matlab environment. Hence it is possible to write the entire code in any of the three environments. Matlab was used to interface the camera and to analyze the acquired images at the same time thus making it a turnkey solution. A screenshot of the Visual $\mathrm{C}++$ demo environment available with the SDK with some of the features explained is shown in Figure 3.1.

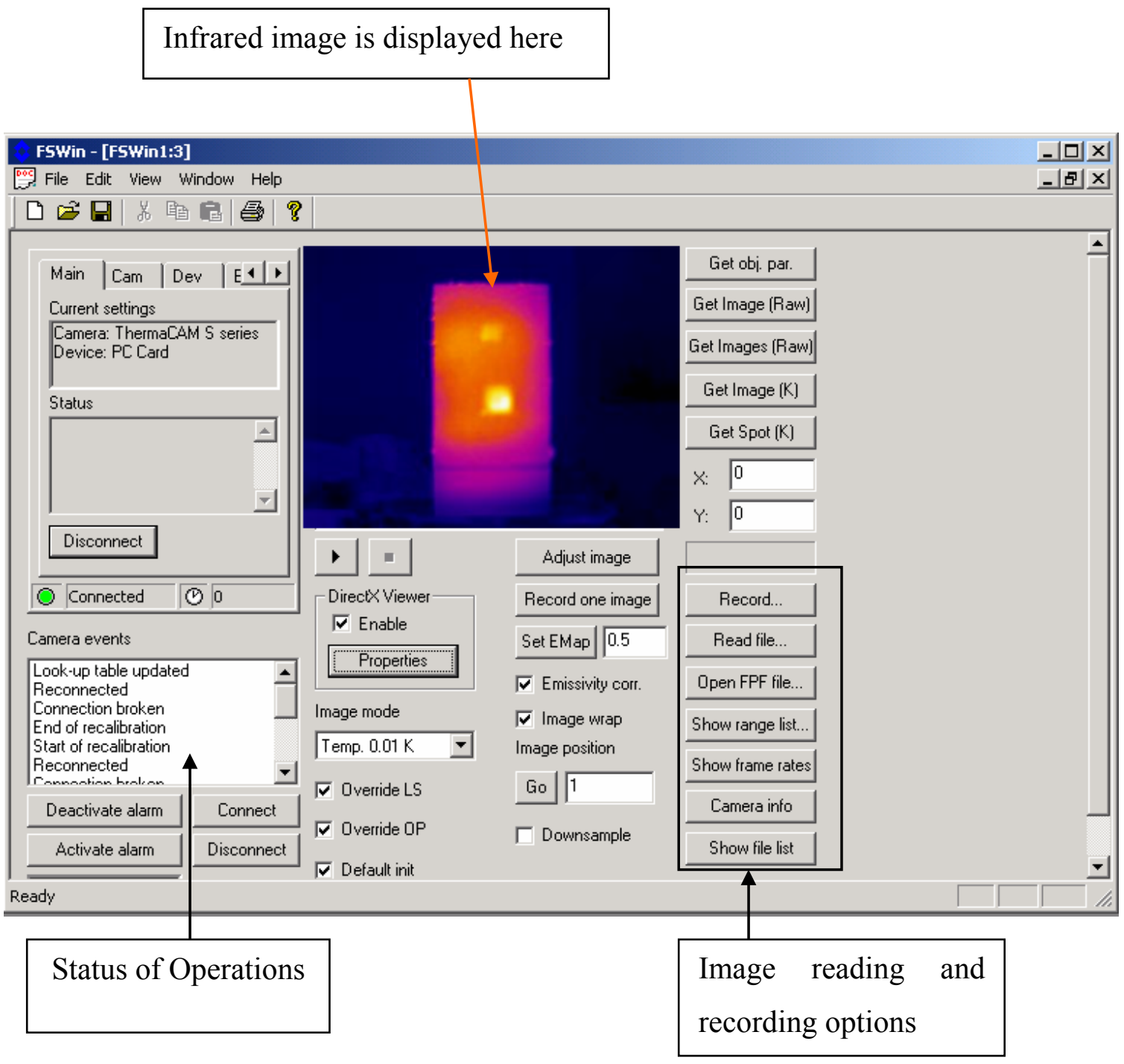

Figure 3.1: FLIR SDK demo screenshot with explanation of the GUI 


\section{CHAPTER 3. AUTOMATED DEFECT DETECTION SYSTEM}

The panel shown on the left in Figure 3.1 is used to connect to the infrared camera before acquiring images. Once connected, various parameters of operation of the infrared camera can be set from the visual interface. Images can be obtained from the infrared camera and stored on the computer. A similar approach is used to develop the prototype turn-key system in Matlab.

\subsubsection{Features of FLIR SDK}

The SDK can be used to switch camera measurement range, control the camera image filtering, view camera status etc. It can also retrieve images and temperature tables and capture an image or a sequence of images. Some of the main features of the SDK are [54]:

- Supports communication and broadcasting via FireWire and Ethernet interfaces

- Gives the user full control of the camera

- Allows the user to set alarm conditions and measurement functions in the camera (ThermoVision ${ }^{\mathrm{TM}}$ A series and ThermaCAM ${ }^{\mathrm{TM}} \mathrm{S}$ series (no alarm) only)

- Allows the user to define $\mathrm{I} / \mathrm{O}$ functionality (ThermoVision ${ }^{\mathrm{TM}} \mathrm{A}$ series)

- Based on ActiveX technology

- Systems proprietary PC-Card frame-grabber, Automation Technology's IRFlashLink, or FireWire and Ethernet interfaces

- Reads from and writes to file in FLIR Systems' proprietary file format and writes to files in FLIR Systems' open floating point format (*.fpf)

- Converts 16-bit raw pixels into temperature pixels and several intermediate types of pixels formats, for maximum user flexibility. Applies to all camera models with temperature measurement capabilities.

- Allows 16-bit temperature linear outputs from ThermoVision ${ }^{\mathrm{TM}}$ A20 M / A40 M FireWire cameras

- Includes method that allows using individual emissivity value correction on any single pixel or condensed measuring value -- e.g. average, minimum etc.

- FLIR Systems' proprietary PC-Card frame-grabber, Automation Technology's IRFlash-Link, or FireWire or Ethernet interface 


\section{CHAPTER 3. AUTOMATED DEFECT DETECTION SYSTEM}

The SDK uses ActiveX technology [54]. The interface developed in the SDK named "FLIR Camera Control" or "LVCam" is used. This control has methods, properties and events by to interact with. The methods and properties allow the user to give commands to the control and to retrieve information from it. The events can tell the user that something important has happened, for instance that the user has pressed the disconnect button. The "Connect" method is used to establish a connection with an infrared camera. After a connection has been established data and commands can be transferred to and from the infrared camera. The syntax of the connect method is:

\section{Status = Object . oonnect $($ camera, port, device, interface, server $)$}

where

Status : return status; $0=\mathrm{OK}$, if nonzero then connection has failed.

camera : type of camera

port : $0=$ Automatic selection of port etc.

device $: 3$ = Firewire (16 bit images)

interface : 3 = FireWire

server : required if camera is connected on Ethernet.

Once these parameters are programmed a valid connection can be established with the infrared camera for enabling digital transfer of images. Similarly the "Disconnect" method disconnects the infrared camera and exits the digital transfer mode. The SDK manual [54] lists all the methods for transferring images, programming and calibrating the camera etc. Using the methods from the SDK integration between the FLIR ThermaCAMS60 Infrared Camera and the Matlab environment was achieved. The GUI for the software designed in Matlab is shown in Figure 3.2. 


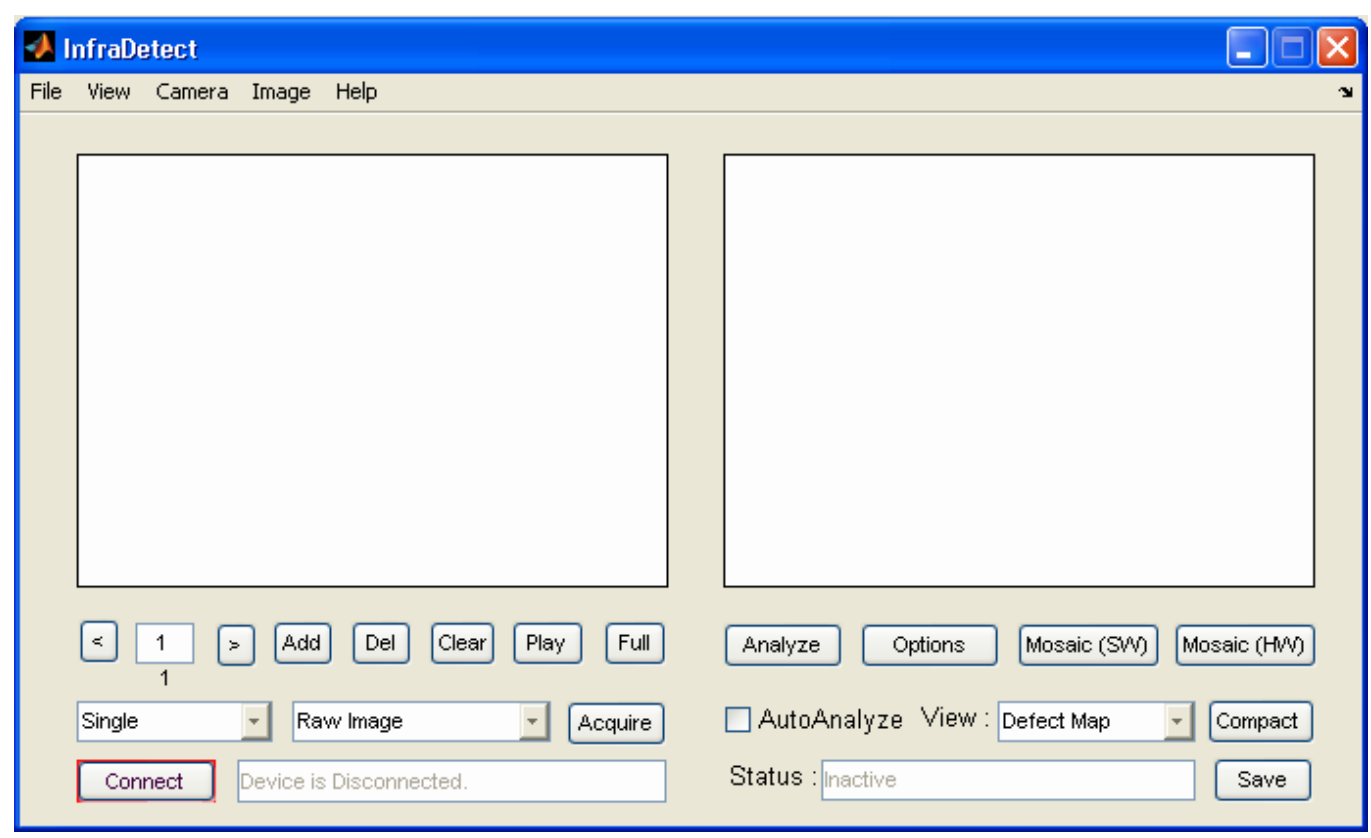

Figure 3.2: GUI for the automatic defect detection software

The GUI can be used to interface with the infrared camera using the "Connect" button show in Figure 3.2 or by using the Connect menu as seen in Figure 3.3. The images acquired/read using this GUI can be analyzed by clicking on the "Analyze" button shown in Figure 3.2. The detailed explanation about the features of the GUI and steps to run the defect detection algorithms are given in Appendix A.

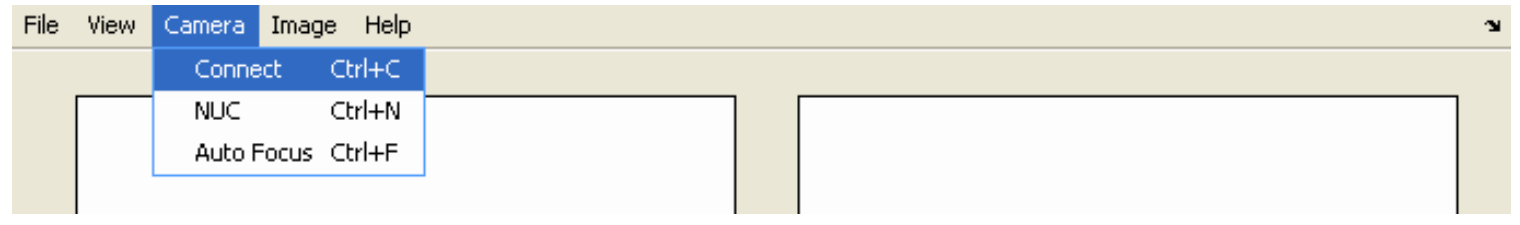

Figure 3.3: Connecting to the infrared camera

A turn-key system was thus developed using the FLIR SDK and the automated defect detection algorithms. The system is able to interface with the infrared camera, capture images and analyze them at the click of a button. 


\subsection{Automated Algorithms for Defect Detection}

Automated defect detection involves the challenge of developing a highly reliable (100\% ideally) infrared image analysis system. One of the problems limiting accurate automated analysis of infrared images is the lack of edge information in infrared images in comparison to the visible image of the same area. The variations in texture and minute details that can be easily seen by the human eye are not visible in the infrared image, essentially because there may not be drastically changing temperature variations over the area under the infrared camera. An example of such an image is shown in Figure 3.4. It is an example of a FRP bridge deck placed in solar radiation on grass. Since the temperature of the bridge deck is much higher than that of grass it is seen in a bright yellow color. The variations in grass background are not highlighted. Also, the complex pattern of grass with minute edge information is not present in the figure. The resulting infrared image has a smooth variation of temperature between the defect and defect free area. Hence a clear distinction between the two may not be determined.

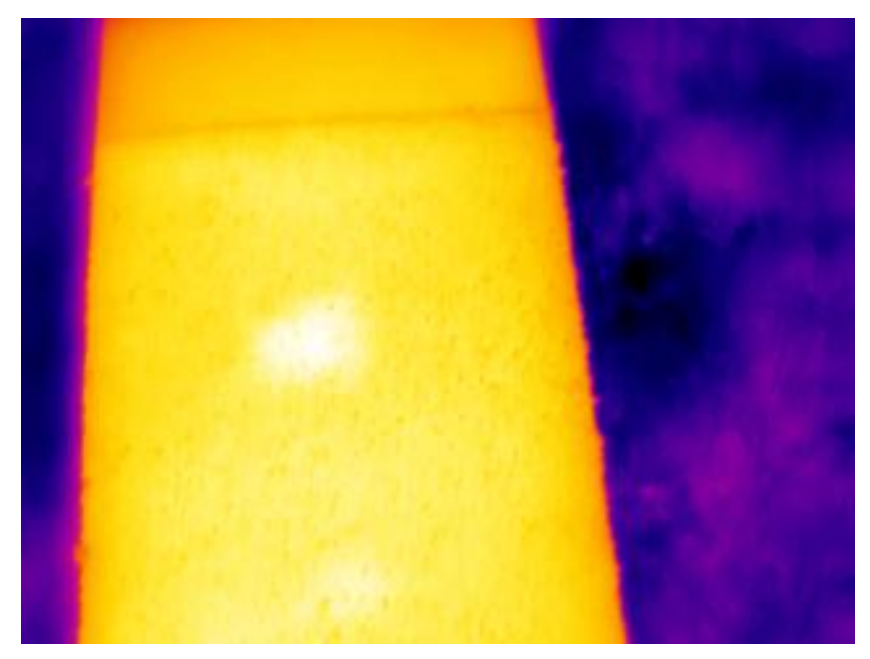

Figure 3.4: Infrared image showing temperature variations [10].

For example, there are two white spots seen in Figure 3.4. The white spot in the center is distinctly visible but has a vague boundary. On the other hand, the white spot at the bottom of the image is barely visible. Both these spots are formed because of thermal differences caused due to subsurface defects. The exact size of the subsurface defects 


\section{CHAPTER 3. AUTOMATED DEFECT DETECTION SYSTEM}

cannot be accurately determined from this image. Also different types of defects can affect the thermal image in different manners. An example is shown in Figure 3.5. It shows an infrared image of bridge deck BD2 that has 3"x3"and 2"x2" water-filled debonds and a 2"x2" air-filled debond. The air filled defect is seen as a high temperature area while the water filled defect is seen as a low temperature area.

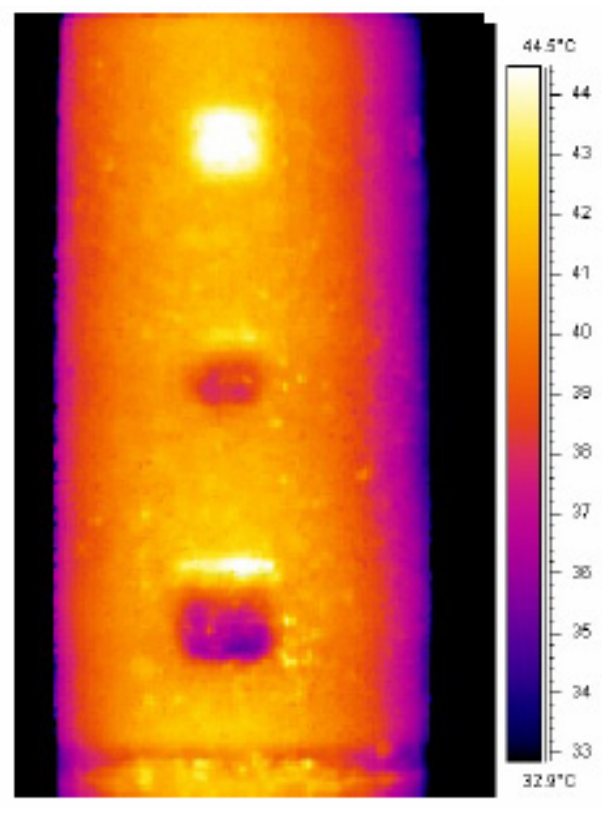

Figure 3.5: Infrared image of bridge deck BD2 [10].

The difference in temperature profiles is because of the presence of water. Water has a high thermal conductivity with respect to the bridge deck and hence cools faster causing a low temperate area. Air on the other hand, air has low thermal conductivity with respect to the bridge deck and appears as a high temperature area. The temperature difference between the defect and non-defect area is proportional to the amount of heat exposure to the deck, size of the defect, depth of the defect etc. Generally, a temperature difference of more than $1.5^{\circ} \mathrm{C}$ between the defect and non-defect area is needed to identify an area as a defect area.

Defects in bridge decks are a naturally formed over a period of operation and are not restricted to a specific shape and size. Hence image processing techniques such as 


\section{CHAPTER 3. AUTOMATED DEFECT DETECTION SYSTEM}

those of object detection are not taken into consideration. Three algorithms developed for automated defect detection are explained in the next sections.

\subsubsection{Morphology Based Algorithms}

An image can be thought of in three-dimensions: the $x$ and $y$-axes represent pixel positions and the z-axes represent the intensity of each pixel. In this interpretation, the intensity values represent elevations as in a topographical map. The areas of highintensity and low-intensity in an image, peaks and valleys in topographical terms can be important morphological features [37] because they often mark relevant image objects. Using morphological processing, the maxima can be used to identify objects in an image. Figure 3.6(b) shows the 3D surface plot of an infrared image of a FRP deck prepared in the laboratory whose original infrared image is shown in Figure 3.6(a). The high temperature regions are seen to have intensity peaks. This fact is exploited to detect defects in the infrared image.

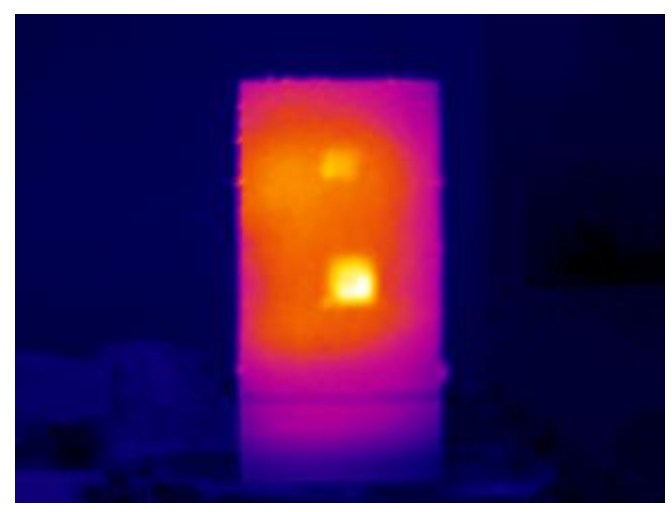

(a)

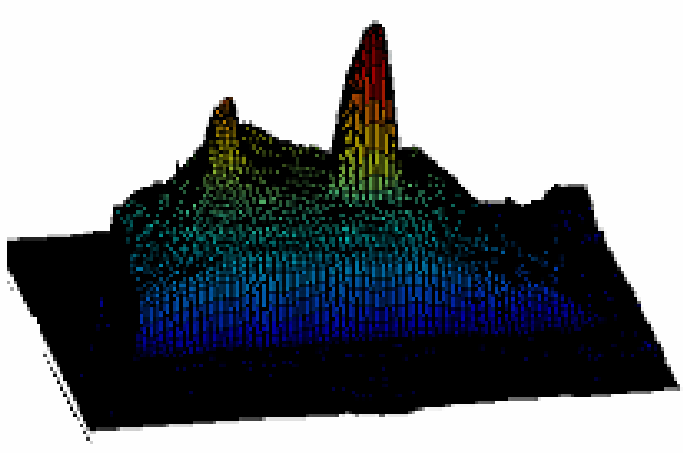

(b)

Figure 3.6: (a) Infrared image of bridge deck BD1a with 3x3" and 2x2" defects [10]. (b) 3D view of the infrared image shown in Figure 3.6(a).

The algorithm involves creating morphological processing related structuring elements and using them to enhance image contrast [37]. Morphological processing refers to operations where an image is filtered using a predetermined shape, referred to as a structuring element to reduce the image to regions of interest. This element is designed 


\section{CHAPTER 3. AUTOMATED DEFECT DETECTION SYSTEM}

around the defect features within the image. First background segmentation is performed to remove the atmospheric area in the image. The processing in the next stages will then be able to concentrate on the bridge deck portion of the image itself.

Next the top-hat and bottom-hat transforms are used to enhance the image. The isolation of gray-value objects that are convex can be accomplished with the top-hat transform as developed by Meyer [43]. It suppresses slow trends in the image to enhance the contrasts of some features in images, according to size or shape criteria. Depending upon whether we are dealing with light objects on a dark background or dark objects on a light background, the transform is defined as [44]:

Light Objects: $\operatorname{TopHat}(\boldsymbol{A}, \boldsymbol{B})=\boldsymbol{A}-\boldsymbol{A}(\boldsymbol{A}$ o $B)$

Dark Objects : $\boldsymbol{T o p H a t}(\boldsymbol{A}, \boldsymbol{B})=(\boldsymbol{A}$ o $B)-\boldsymbol{A}$

where 'o' represents the opening or closing morphological operation.

Similarly a bottom-hat transform is used to isolate the gray-value objects that are concave. The top-hat image and bottom-hat image are used to obtain an image with enhanced using the formula:

\section{Enhance image $=$ Original + Top-hat - Bottom-hat}

A decorrelation stretching procedure is then applied to enhance the contrast in the image. Decorrelation stretching process is a color enhancement technique that is based on a principal component transformation of correlated multispectral image data [45]. Generally, highly correlated image channels, such as the Red, Green and Blue (RGB) channels in remotely sensed infrared images show subtle differences well, but these colors are not clearly related to the different surface types. Simple contrast exaggeration can be used to expand the range of intensities of highly correlated images, but contrast exaggeration does little to expand the range of colors. To enhance the color in highly correlated images, one needs to selectively exaggerate the least correlated portion of the spectral data, i.e. decrease the correlation. Decreasing the correlation of spectral data 


\section{CHAPTER 3. AUTOMATED DEFECT DETECTION SYSTEM}

corresponds to exaggerating the color saturation without changing the distribution of hues (or relative color composition).

The decorrelation stretching process involves three fundamental steps [46]: First, a principal-component transformation is applied with the rows and columns of the eigenvector matrix transposed. Second, contrast equalization is applied by a Gaussian stretch, so that histograms of all principal components approximate a Gaussian distribution of a specified variance. Third, a coordinate transformation that is the inverse of the principal component rotation is applied so that the data are projected in their original spectral channels, using eigenvectors as weightings for each principal component. This inverse operation maximizes the spectral seperability of different surface types in the restored spectral channels generating a decorrelation stretched image. The implicit assumption in this technique is that the differences in color intensities are real and not noise or processing artifacts. The algorithm single-mindedly produces a color enhanced output image. Detail explanation about the working of this algorithm is given in [47].

A global thresholding procedure is applied on the decorrelation stretched image to generate a binary defect map. Finally the detected defects are verified to be true by comparing them with some criterions found heuristically. The size of each defect is compared with a threshold. If the size is less than 50 pixels or is more than 8000 pixels the region is not considered as a defect area. This assumption seems to be valid as small surface irregularities such as the presence of pebbles can cause false detection of defects which are small in size. On the other hand abnormalities such as reflections from the sun cause a large portion of the exposed area to be designated as defect. These errors are checked and removed in this stage. The average and standard deviation of the defect area are also calculated and compared with that of the surrounding area to remove any areas that may be falsely identified as defects.

After the spurious defect removal procedure, a binary defect map is obtained with defect areas segmented in white. The border of the detected defects is marked in the original image to obtain a color infrared image with the detected defects. A sample run of the algorithm with outputs from different stages is shown in Figure 3.7. 

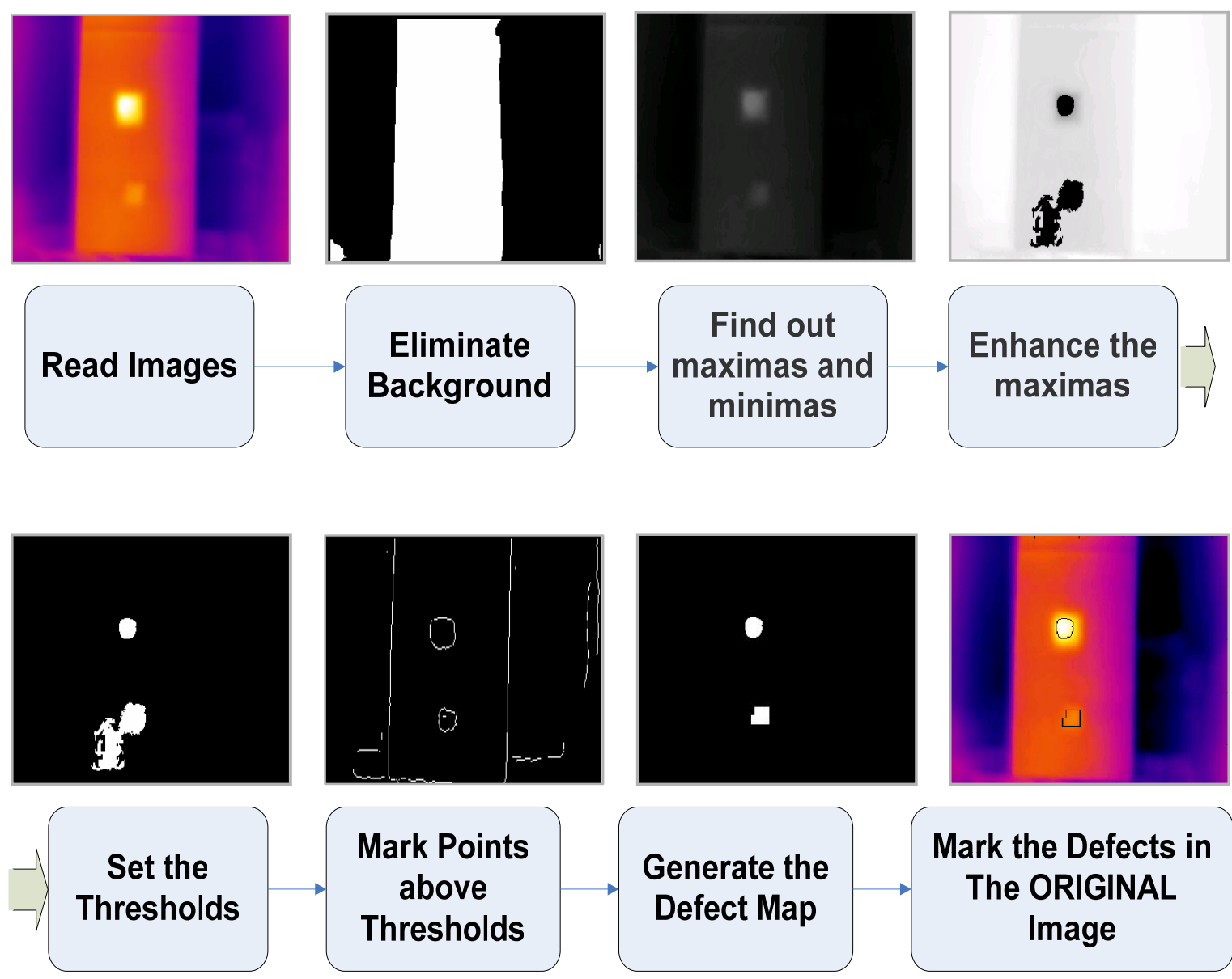
Mark the Defects in The ORIGINAL Image

Figure 3.7: Various stages of morphological defect detection algorithm.

\subsubsection{Fuzzy Clustering Algorithm}

The second approach for automated defect detection was developed using a fuzzy clustering algorithm. Clustering of numerical data forms the basis of many classification and system modeling algorithms. The purpose of clustering is to identify natural groupings of data from a large data set to produce a concise representation of a system's behaviour. Here the purpose is to identify if a group of pixels in the image belong to any defect region.

First background elimination is applied to remove pixel variation in the background. This helps is assigning just one cluster to all the background pixels. The remaining clusters are used to segregating the image pixels into defect and non-defect regions. The fuzzy c-means is followed by a decorrelation stretching procedure explained 


\section{CHAPTER 3. AUTOMATED DEFECT DETECTION SYSTEM}

in Section 3.4.1. This will enhance the color contrast in the image, thus increasing the accuracy of the fuzzy clustering procedure.

Then fuzzy c-means clustering is performed on the enhanced image. Fuzzy cmeans (FCM) is a data clustering technique wherein each data point belongs to a cluster to some degree that is specified by a membership grade. This technique was introduced as an improvement on earlier clustering methods. It provides a method that shows how to group data points that populate some multidimensional space into a specific number of different clusters. An initial guess of the cluster centers is taken. The initial guess for these cluster centers is most likely incorrect. Additionally every data point is assigned a membership grade for each cluster. By iteratively updating the cluster centers and the membership grades for each data point, the cluster centers are moved to the right location within a data set. This iteration is based on minimizing an objective function that represents the distance from any given data point to a cluster center weighted by that data point's membership grade. An image segmentation procedure using fuzzy clustering has been exploited by researchers such as Horvath [48]. Modified versions of the fuzzy clustering algorithm have also been used in the field of medical imaging by [49].

At the end of the fuzzy clustering every pixel is assigned a membership grade with the number of clusters assigned. The number of clusters can be chosen by the user or the algorithm chooses between 6 to 9 clusters for the entire image based on the image characteristics. For a pixel with membership grades assigned to each cluster, the cluster having the highest grade is chosen as cluster to which the pixel belongs. In this way all the pixels are assigned to one of the clusters. An example of a cluster map formed in such a way is shown in Figure 3.8.

Each color in Figure 3.8 corresponds to one cluster. The black color corresponds to background pixels while the cluster represented by blue color occupies most of the pixels corresponding to the defect area. Some of the pixels in the orange colored cluster are correspond to the true defect area. This pattern of concentrating the defect area into one or two clusters is prominent in all the infrared images testes using the fuzzy clustering algorithm. 


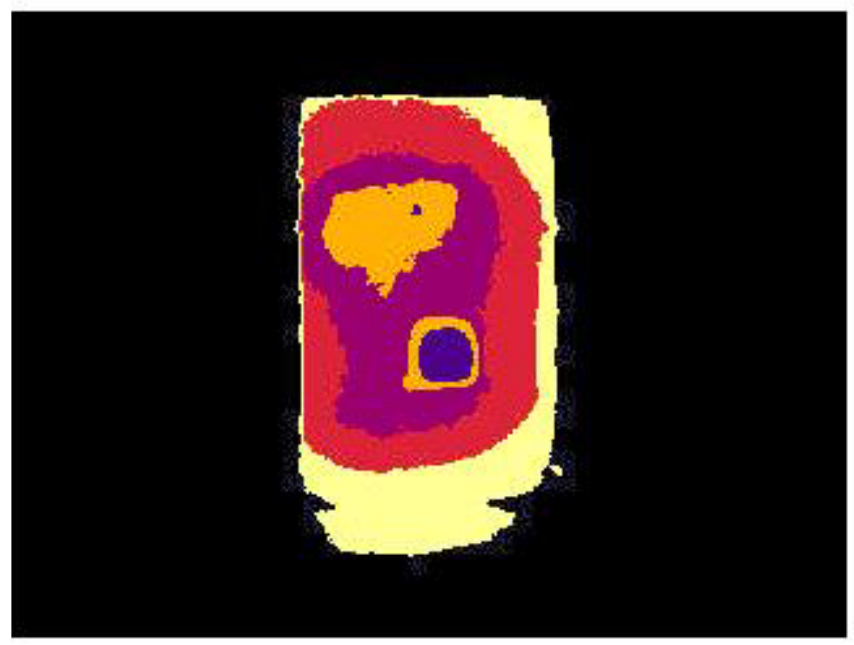

Figure 3.8: Cluster map of the image shown in Figure 3.6(a).

The next step is to determine the defect area from the clustered image or cluster map. As explained previously, it was experimentally found that most of the defect pixels are generally grouped into one cluster or sometimes into two clusters. The cluster with the highest average pixel intensity or the highest average temperature corresponds to the true defect area. The next best cluster (the cluster having the second highest average pixel intensity or average temperature) may have some pixels corresponding to true defect area. A threshold value equal to $80 \%$ of the minimum pixel intensity or minimum temperature of the defect cluster (cluster with highest average pixel intensity) is calculated. The $80 \%$ cut-off value was empirically found to give accurate defect detection results. This threshold is then applied to the next best cluster.

A defect map is obtained based on the threshold calculated. The defect map is then passed through the defect verification procedure explained in Section 3.4.1 to generate a true defect map. The defect map is overlaid on the original image to enable easy segregation of the defect areas. A sample run of the fuzzy algorithm with outputs from various stages is shown in Figure 3.9. The figure shows various stages of the algorithm; background elimination followed by fuzzy clustering after which thresholds are calculated and a defect map is obtained. 

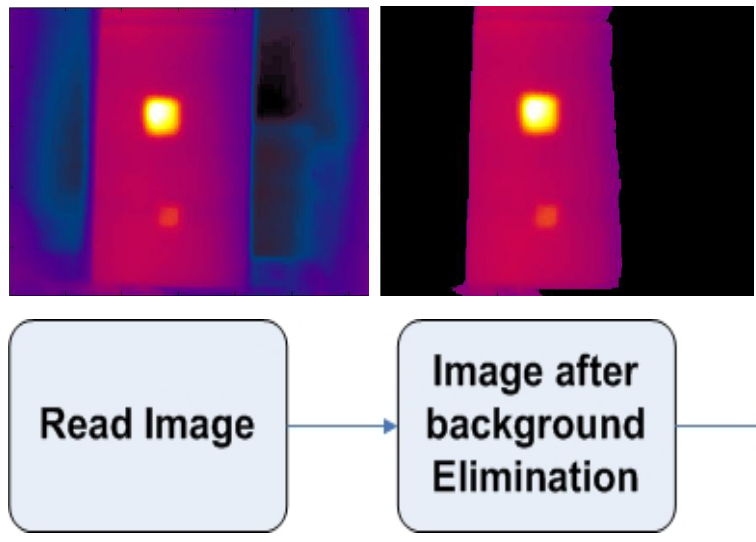
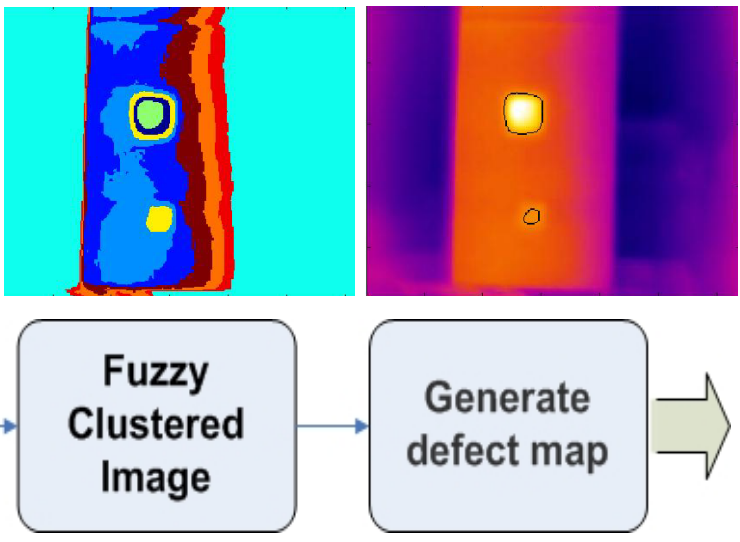

Figure 3.9: Various stages of fuzzy clustering based defect detection algorithm.

\subsubsection{Combination Algorithm}

The combination algorithm, as the name suggests was an effort to access the advantages of both the algorithms (morphological and fuzzy clustering). The morphological algorithm uses a top-hat and bottom-hat transform to enhance the original image. This increases the accuracy of defect detection. The clustering algorithm segregates the defect areas from non-defect areas with ease. Hence the two are merged to form the combination algorithm. The block diagram of the combination algorithm is shown in Figure 3.10.

The background separation, image enhancement and decorrelation stretching blocks are the same as used in the morphological algorithm. They are marked by a brown rectangle in Figure 3.10. The explanation for these steps is given in Section 3.4.1. The output of these morphological processing steps is an enhanced infrared image that is suitable for defect detection. This enhanced image is given to a fuzzy clustering procedure marked by a green rectangle in Figure 3.10. The steps described in Section 3.4.2 for formation of the cluster image followed by calculation of thresholds to obtain a defect map and then remove spurious defects from the defect map are utilized in this part of the algorithm. Finally the defect map is overlaid on the original image to obtain an image with the defect areas marked by a black border. 


\section{Morphological image enhancement}

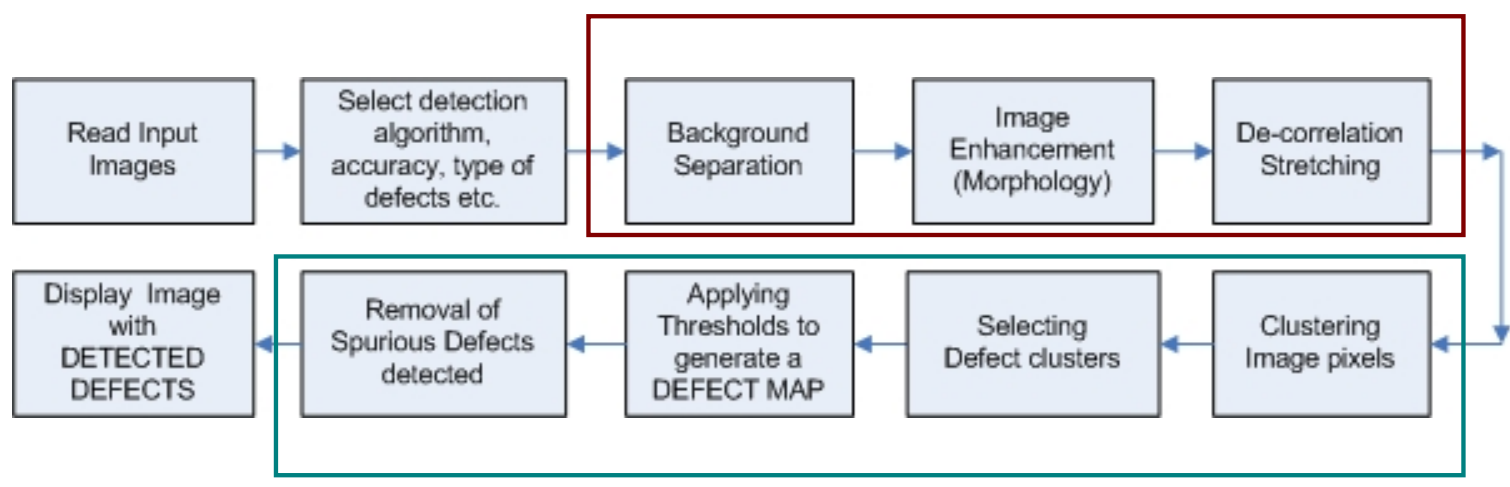

Fuzzy Clustering

Figure 3.10: Block diagram of the combination algorithm.

\subsubsection{Comparison of the Algorithms}

The entire implementation was done in the Matlab environment. The morphology based algorithm was found to be the fastest and could analyze an image for defects in approximately 1.25 seconds on a Pentium M 1.6GHz processor. This algorithm has the least complexity but the sensitivity of defect detection is limited. Some of the defects are detected with improper dimensions and defects having less temperature difference with respect to surrounding areas are not detected with ease.

The fuzzy clustering and the combination algorithm approximately required 18 and 20 seconds respectively. The best results are obtained by the combination algorithm. Since the software was developed in the Matlab environment the execution time is increased. There should be a substantial increase in the speed if the software is ported to a $\mathrm{C} / \mathrm{C}++$ code making real time defect detection possible.

\subsection{Mosaicking of Infrared Images}

A single infrared covers only a small area of the entire bridge deck. As the distance of the camera from the bridge deck increases the accuracy of defect detection is adversely affected since the number of pixels representing the defect decrease. Even though defect detection in a single image is achieved, the corresponding physical location 


\section{CHAPTER 3. AUTOMATED DEFECT DETECTION SYSTEM}

of the defect should be known. In order to ascertain the actual location all the images of the bridge deck with the detected defects have to be combined to form a single composite deck image. The single composite image covers the bridge deck area covered by all the infrared images combined. Hence the presence of a defect in the composite image can be

directly correlated to a physical location on the bridge deck. This process of joining two or more images to form a composite image is known as image mosaicking.

Mosaicking of infrared images provide a method to obtain a single image representation of the entire deck. Defect detection can then be carried out on each infrared image. The output image will be the original image with the defects marked, if any. Many such images will be joined together to obtain the "image mosaic". The problem of image mosaicking is a combination of three problems [50]:

$>$ Correcting geometric deformations using image data and/or camera models.

$>$ Image registration using image data and/or camera models.

$>$ Eliminating seams from image mosaics.

Figure 3.11 shows the setup for capturing infrared images of the bridge deck that can be used for image mosaicking. It consists of a cart with an infrared camera mounted on top. The cart is moved forward and images are captured in a sequence. Since the camera is mounted on the cart the images are captured by keeping the angle and rotation of the camera with respect to the bridge deck constant. The camera is moved only in the horizontal (X) and vertical (Y) direction. Two mosaicking approaches explored here are [51]:

Hardware Assisted Mosaicking: In this approach the cart is assumed to have sensors that give displacement measurement between two consecutive images. These displacement parameters are used to join the images together to form a composite image.

Software Assisted Mosaic: This approach involves calculating the displacement parameters automatically by identifying similar features between two consecutive images. The displacement parameters are used to join the two images to form an 
image mosaic. This method is computationally complex and not suitable for real time operation.

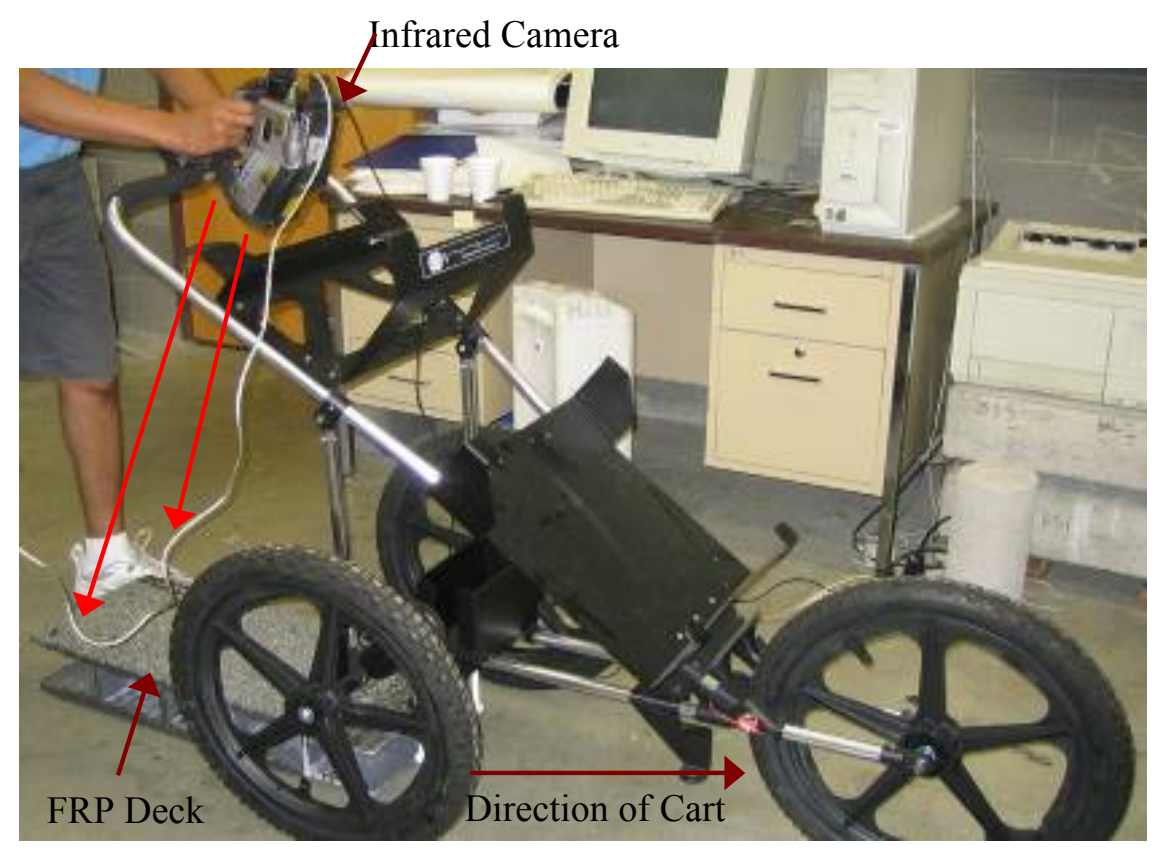

Figure 3.11: Setup for infrared image mosaicking.

\subsubsection{Hardware Assisted Mosaicking}

The generic setup for infrared image mosaicking is shown in Figure 3.11. Displacement sensors can be added to the cart seen in the figure. The sensors will trigger an infrared image capture from the camera after a specific distance is traveled. The user can specify before hand the number of images to be captured in the vertical (Y) direction and in the horizontal (X) direction. The algorithm for hardware assisted image mosaicking is shown in the flow chart in Figure 3.12(a). Because of the presence of sensors, exact displacement between two consecutive infrared images is known before hand eliminating the requirement of complex algorithms. Thus the job of the system is to capture an image and directly join with the previous image till the entire bridge deck area is traversed. Hence real time implementation becomes feasible.

An example of a hardware assisted mosaicking system is shown in Figure 3.12(b) with the detected defects. Hence in order to demonstrate the concept the user is asked to 


\section{CHAPTER 3. AUTOMATED DEFECT DETECTION SYSTEM}

input the number of images in the $\mathrm{X}$ and $\mathrm{Y}$ direction and image capture is triggered manually. Let Xpar and Ypar represent input parameters set by the user. The captured images are joined as per Xpar and Ypar. The cart is assumed to go forward "Ypar" times followed by one step in the $\mathrm{X}$ direction. Then it traverses in the backward direction till it reaches one step besides the starting position. At this point it again makes a $U$ turn and the process continues till the number of steps in the $\mathrm{X}$ direction are completed. It is graphically shown in Figure 5(b) with Ypar $=4$ and Xpar $=3$. Thus 12 images are joined together to form a composite image mosaic of the entire GFRP bridge deck.

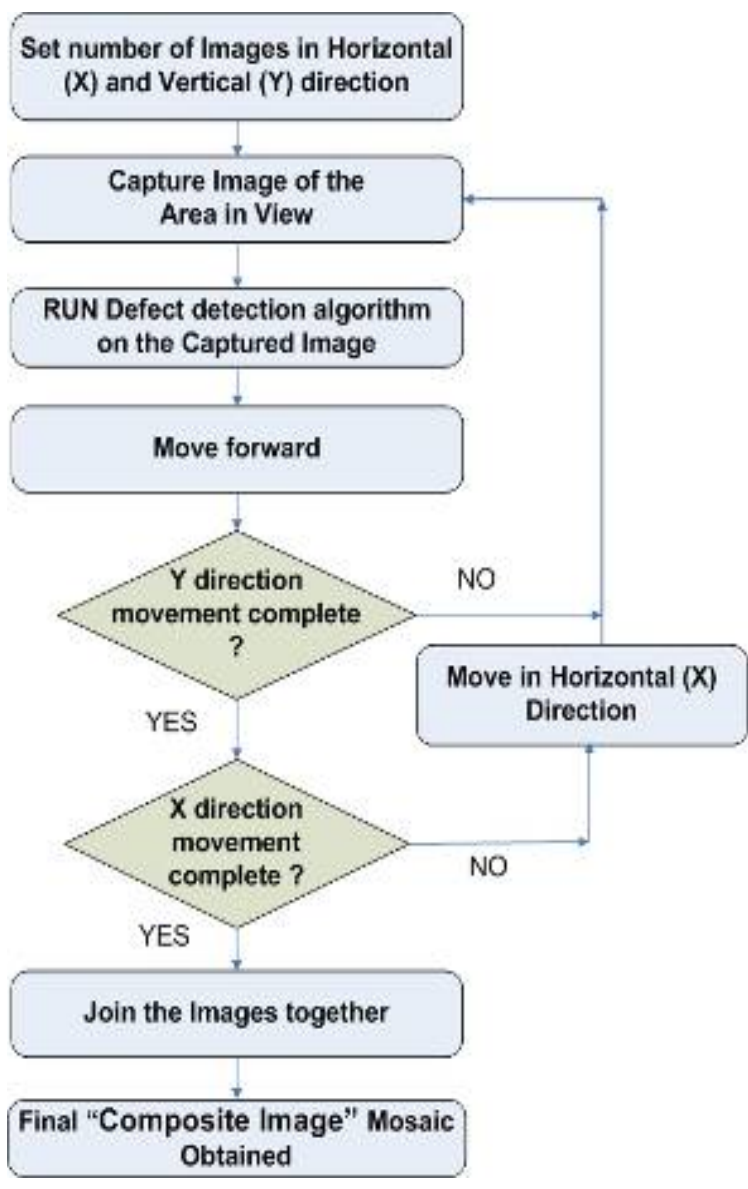

(a)

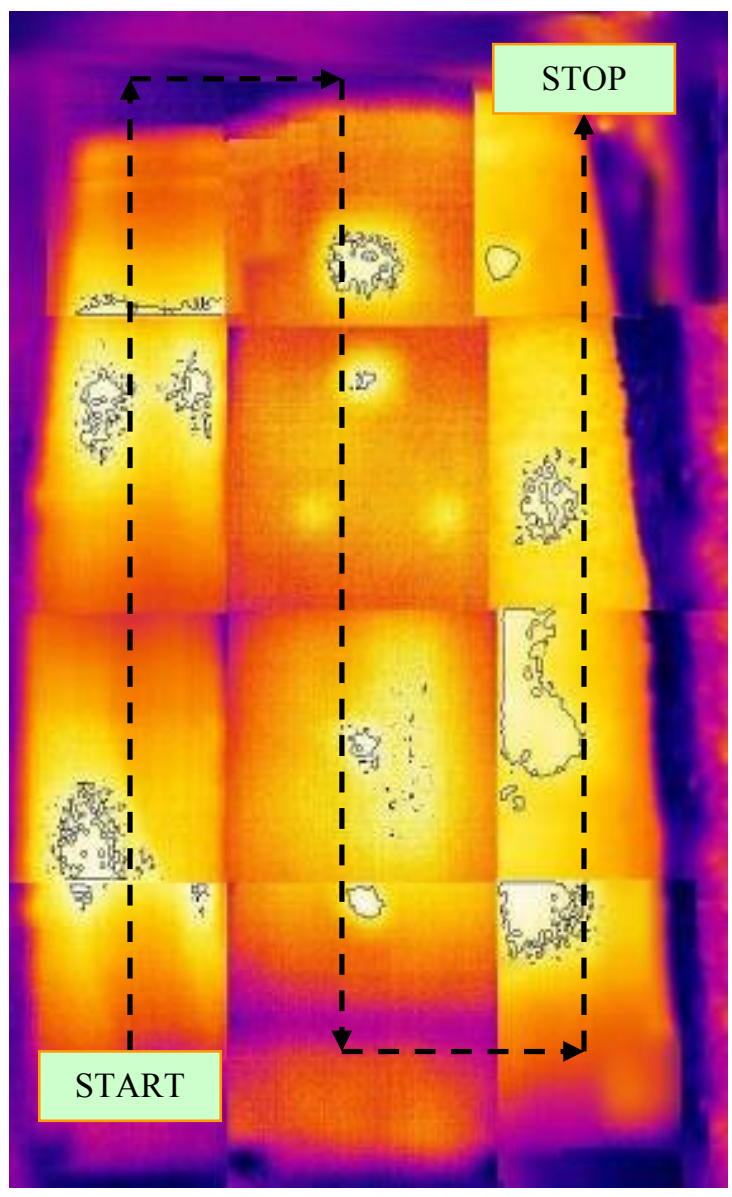

(b)

Figure 3.12: (a) Algorithm for hardware assisted image mosaicking. (b) Example of a hardware assisted mosaic with the detected defects. 


\section{CHAPTER 3. AUTOMATED DEFECT DETECTION SYSTEM}

The advantage of this approach is that need for calculating the displacement parameters between two images is eliminated. Hence once the hardware setup is designed and programmed, the system will be able to capture infrared images at regular intervals. Thus real time operation of image capture, analysis and mosaicking will be possible.

\subsubsection{Software Assisted Mosaicking}

Software assisted mosaicking is accomplished by automatically determining the displacement between two consecutive infrared images. Since the camera angle is fixed, a euclidian similarity transform can be used to determine the translation and rotation between two images. Control points are selected either on the basis of the grey level value only or by using the Harris corner detector [52]. In the grey level approach it was found experimentally that the corners or curves near the defect regions are constituted by certain grey level values. Edge detection is used to find out the edge/curve points in the highest intensity areas of the images. These points from two or more images are used as control points. If more than ' $\mathrm{N}$ ' points are found, minimization of control points is done by assigning more importance to the points near the high intensity region of the image. This approach was found to work well on some images but was not universally acceptable. The corner detection approach using the Harris corner detector [52] was found to be more robust with respect to the grey level based methods. It was used to find a set of control points for the two images to be mosaicked. An example of such a set of control points for two images to be mosaicked is shown in Figure 3.13(a) and (b).

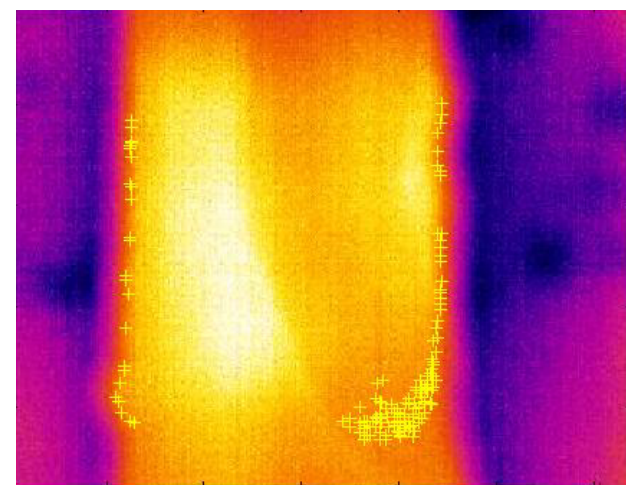

(a)

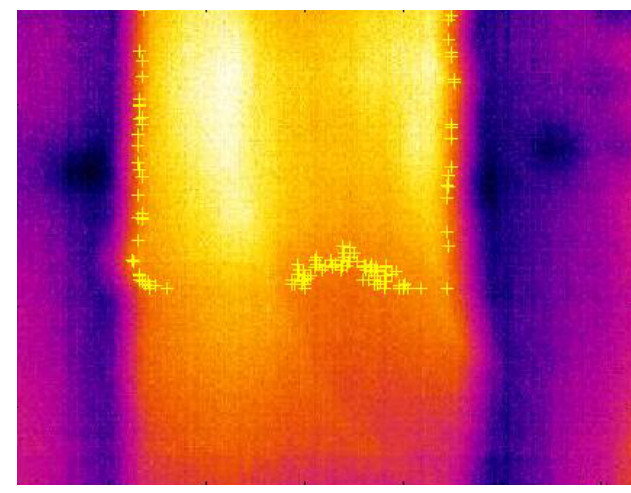

(b)

Figure 3.13: (a)(b) Infrared images to be mosaicked along with the set of control points 


\section{CHAPTER 3. AUTOMATED DEFECT DETECTION SYSTEM}

Next, the set of control points form the two images in consideration are given to a point pattern matching algorithm. The algorithm selects two random points from image in Figure 3.13(a) and tries to match the features of the line (i.e. length and angle with respect to the origin) with point pairs from image in Figure 3.13(b). A restriction is applied during the selection of point pairs from an image. The restriction is that the line segment formed by the two points should be at least 50 pixels in length and have an angle between $\pm 40^{\circ}$ where the $\mathrm{X}$ axis is considered as the $0^{\circ}$ axis. This helps in reducing the number of control point pairs that have to be evaluated to obtain the transformation parameters between the two images. For a given point pair in the image in Figure 3.13(a) all the point pairs in image Figure 3.13(b) having similar length as the point pair in Figure 3.13(a) are found. The difference between their characteristics (angle and length) is used to estimate the Euclidian warping (Euclidian similarity transform) parameters. Euclidian warping is a type of image warping that changes the spatial configuration of an image. It involves four parameters: [s $\alpha$ tx ty] where

$\mathrm{s}=$ scale parameter (in our case the scale is assumed to be 1)

$\alpha=$ angle of rotation between the two images.

tx ty $=$ the $\mathrm{X}$ and $\mathrm{Y}$ translation parameters between the two images.

A point $[\mathrm{x} y]$ in an image is transformed to another point [ $\left.\mathrm{x}^{\prime} \mathrm{y}^{\prime}\right]$ by the equation shown below.

$$
\left[\begin{array}{c}
x^{\prime} \\
y^{\prime} \\
1
\end{array}\right]=\left[\begin{array}{ccc}
s \cos \alpha & s \sin \alpha & t_{x} \\
-s \sin \alpha & s \cos \alpha & t_{y} \\
0 & 0 & 1
\end{array}\right] \cdot\left[\begin{array}{l}
x \\
y \\
1
\end{array}\right]
$$

The same equation can be used to warp a set of points or the entire image. In our case, it is used to warp the set of control points of Figure 3.13(b). From the pair of selected control points the three parameters for Euclidian warping, i.e. [ $\alpha$ tx ty] are found and the new set of control points of Figure 3.13(b) is obtained. The new set represents the new location of these points after it us translated by $t x$, ty and rotated by an angle $\alpha$. If the values correspond to the actual transformation that has taken place there will be a 
maximum overlap between the two sets of control points. As the estimated values of $[\alpha t x$ ty] deviate from the true value, the overlap decreases. An actual "score of overlap" between the control point of Figure 3.13(a) with respect to the newly estimated control points of Figure 3.13(b) is obtained. A pair of matching control points selected from the set of points for the two images in Figure 3.13 is depicted in Figure 3.14.

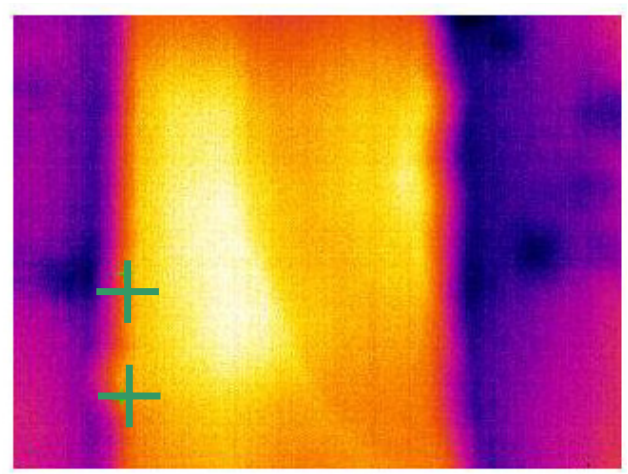

(a)

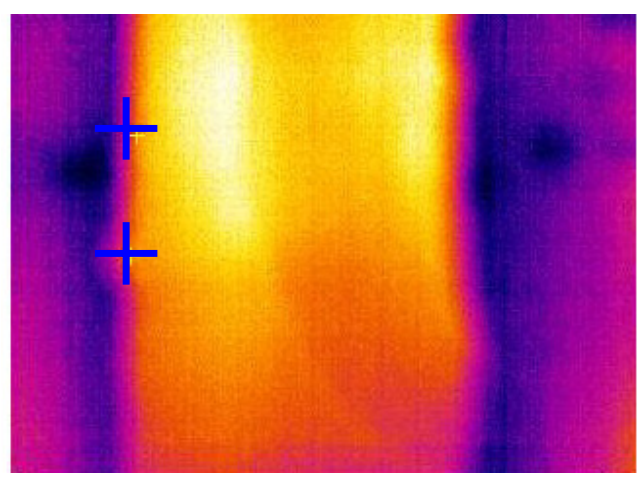

(b)

Figure 3.14: (a)(b) Matching pair of control points among the images shown in Figure 3.13(a)(b).

This process of selecting two control point pairs, finding [ $\alpha t x t y]$ between them, warping the control points of Figure 3.13(b) and calculating a match score is repeated for all valid pairs of control points in the two images. The transformation parameters that yield the highest matching score is chosen as the best match of the actual transformation. The algorithm described above is shown in the flow chart in Figure 3.15. The result of running the software assisted mosaicking algorithm on the images shown in Figure 3.13 is depicted in Figure 3.16. 


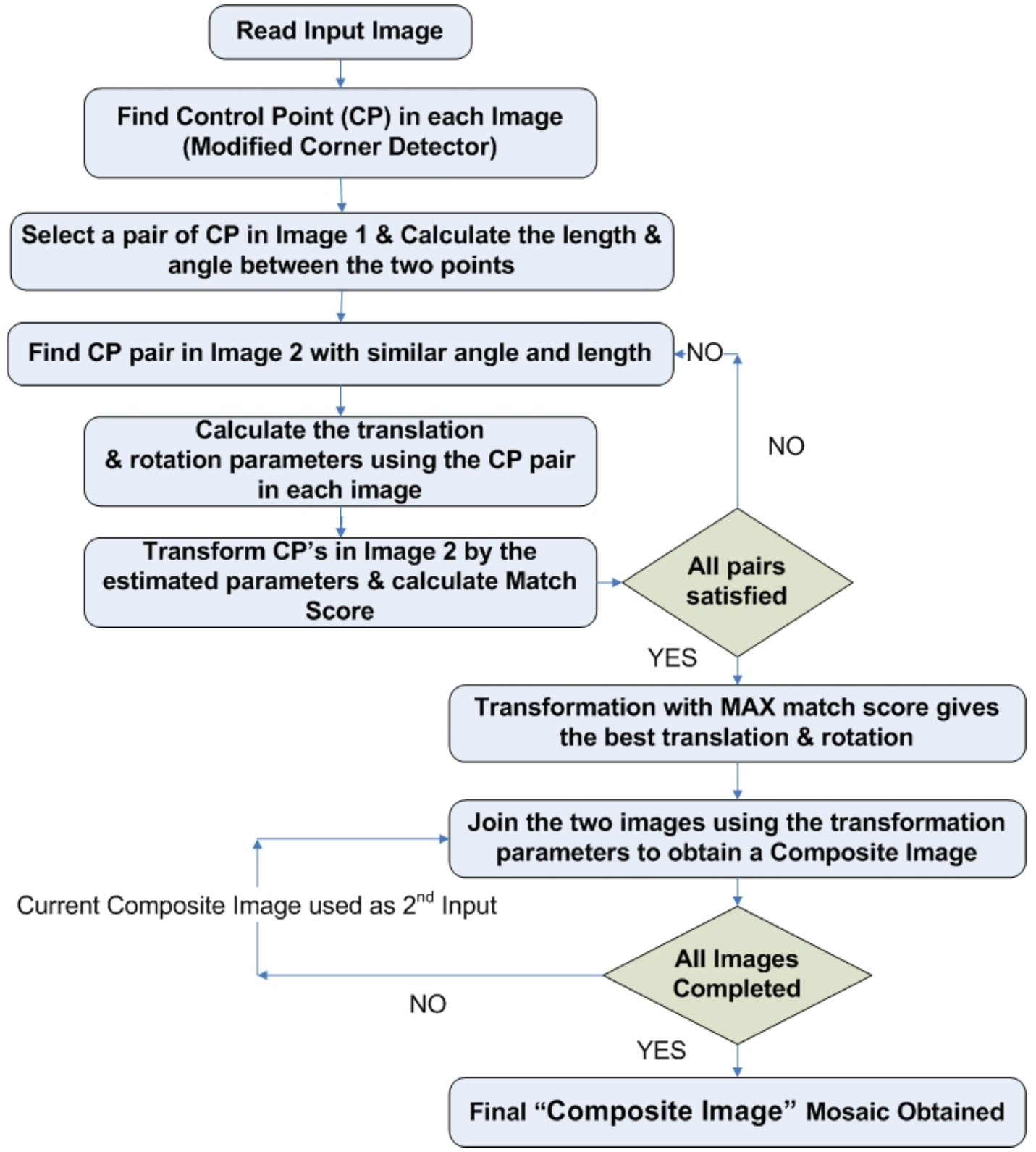

Figure 3.15: Algorithm for software assisted Image mosaicking. 


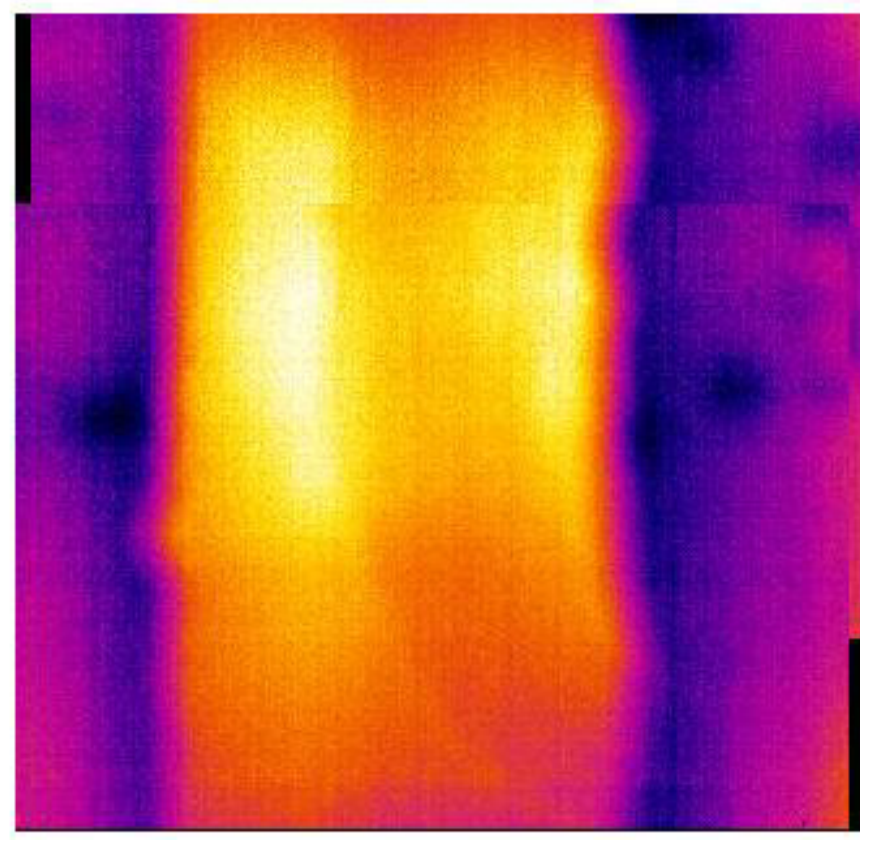

Figure 3.16: Example result of the software assisted mosaicking algorithm.

\subsubsection{Comparison on the two Approaches}

The advantage of the software assisted approach is that it can be used to calculate a mosaic for any given set of input images irrespective of the camera angle and rotation between two consecutive images. However, the approach is computationally complex. It takes 20 seconds to mosaic two images using the developed code. Hence it is not suited for real time implementation. Also, it has limitations in accurate mosaicking because of the nature of infrared images from GFRP decks. The images obtained are smooth with less information about corners or edges. This information is needed to detect control points robustly. Hence even with small displacement between two images the control points detected can be different. This reduces the accuracy of the process of estimating translation and rotation parameters. Hence a faulty mosaic map may be obtained in such a case. On the other hand the hardware assisted approach does not need the calculation of the displacement parameters between two images as sensors are used for the process. Hence the hardware has to only automate the sequence of image capture, reading feedback from the sensors, and mosaicking the images to generate a composite image 
map. This process can be easily implemented to build a real time system for mosaicking infrared images.

\subsection{Summary}

The research focused on the development of a turn-key system that is able to interface with the infrared camera and automatically analyze defects in infrared images. Different algorithms that are able to segregate the defect areas and the non-defect areas from the infrared images are developed. The algorithms are able to detect both the water filled and air filled defects in infrared images. The results or running the defect detection algorithms under various conditions are enlisted in Chapter 4.

The advantage of using image mosaicking for joining infrared images to form a composite image map was explored. Software and hardware assisted approaches for infrared image mosaicking are explored. Software assisted approaches are found to be time consuming and may not be suitable for real time applications. Hardware assisted approach has the advantage of being computationally efficient and can be used in real time operation with ease. 


\section{Chapter 4}

\section{Experimental Setup and Results}

\subsection{Bride Deck Manufacturing}

FRP components are being increasingly used in the repair and construction of civil engineering structures as explained in Section 1.2. Glass Fiber Reinforced Polymer (GFRP) bridge decks manufactured at Bedford Reinforced Plastics were used in this study. They are manufactured into different shapes and sizes by pultrusion process. A detailed explanation about the pultrusion process is given in [24]. Photographs of a bridge deck coming out of the pultrusion process at Bedford Reinforced Plastics are shown in Figure 4.1.

These bridge decks could develop subsurface defects (debonds and delaminations) during manufacturing, transportation, construction or during service. Such subsurface defects adversely affect the load carrying capacity of the deck and cause a decrease in the structural integrity. Hence there is a need to inspect FRP bridge decks at periodic intervals and monitor the growth of these subsurface defects. Since there are very few FRP bridges in operation defects are artificially inserted in the manufactured bridge decks for the purpose of a laboratory study. This was performed by the work of Vasudevan A., Roy M., Halabe U. and Hota G. [10,21,4,22]. The process of artificial insertion of defects of various sizes inside the FRP decks is explained below. 

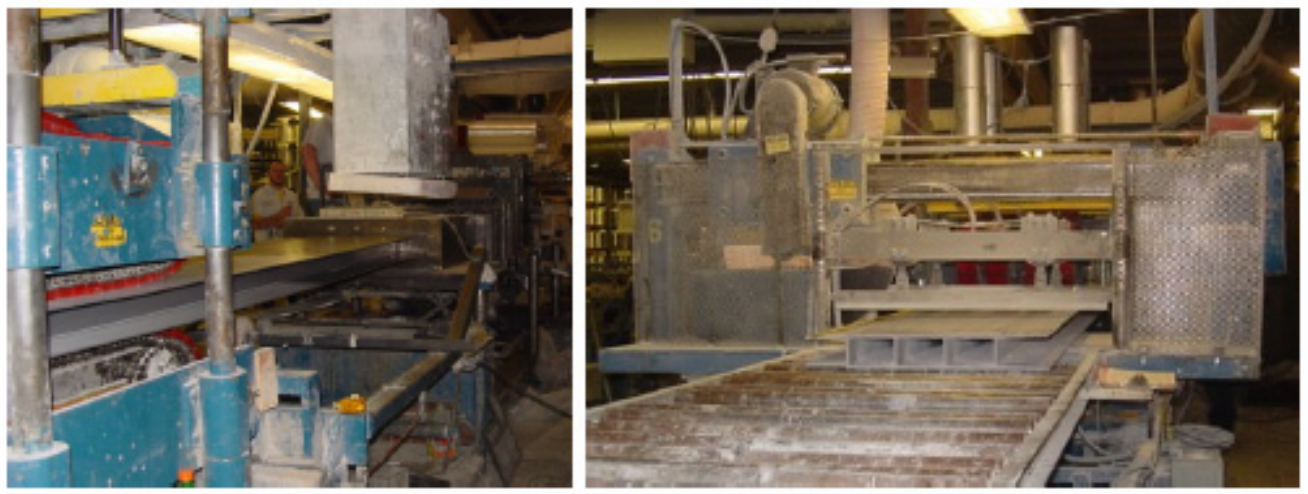

Figure 4.1: Photographs showing manufacturing of an FRP bridge deck in a factory using the pultrusion process at Bedford Reinforced Plastics [10].

\subsection{Artificial Generation of Defects [10]}

Two types of defects namely debonds and delaminations were inserted below the surface of the FRP bridge decks. Debonds refer to the subsurface defects that are present at the interface between the wearing surface layer and the underlying FRP deck or between the composite wrap and underlying component. Delaminations refer to the defect present within the top or bottom flange of the deck material. The air-filled debonds were made by joining two polypropylene sheets with an enclosed air pocket in between them. These debonds were sealed in between two latex sheets so that the air is fully trapped inside. In a similar manner water-filled debonds were made by enclosing water within super-strength plastic sheets.

A milling machine was used to mill (or cut) rectangular portions of required sizes on the flange joint area of a bridge deck module. These delaminations of various plan sizes and thicknesses were created in the middle of the flange joint area (top flange) in one of the modules before joining two modules together. The delamination was covered with wax paper so that the glue could not get into the delamination with the air gap intact. The two deck modules were then joined together using a structural adhesive called Pliogrip. The delaminations were thus embedded within the flange-flange junction of two contiguous modules at an average depth of 0.32 " $(8 \mathrm{~mm})$ from the top surface. Figure 4.2(a) and (b) show the photographic view of the specimens with the embedded delaminations [10]. 


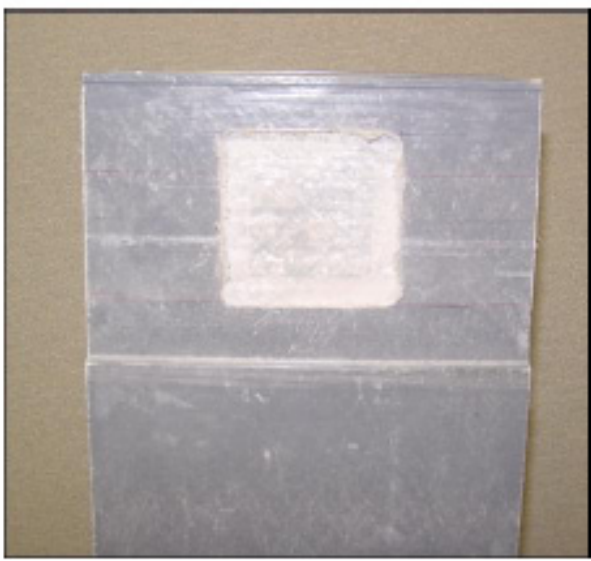

(a)

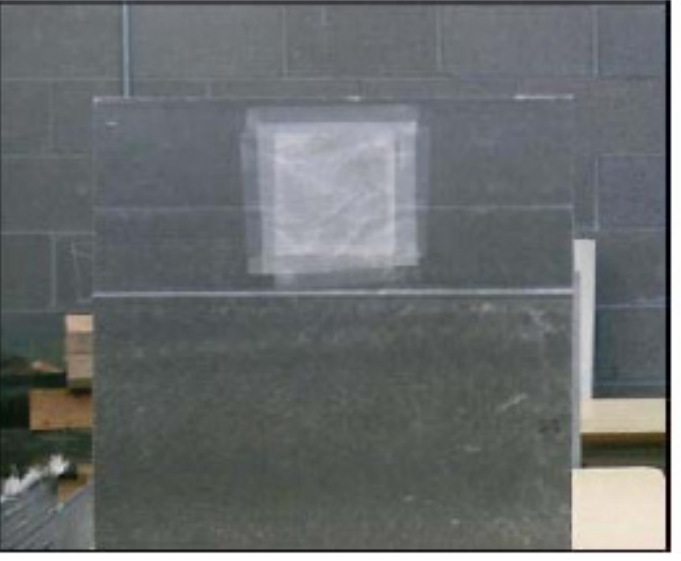

(b)

Figure 4.2: Close up view of the 3 " $x 3$ " delamination, created at the flange-flange junction of a module (a) without wax paper (b) with wax paper [10].

\subsection{Description of Specimens [10]}

Many FRP bridge deck specimens with varying defects were prepared in the laboratory in the work by $[10,21,4,22]$. Their brief description is given below.

\subsubsection{Bridge deck specimen BD1 and BD2}

The specimen BD1 consisted of GFRP bridge deck module of size 24"x12" and overall depth of $8 \%$. The top and the bottom flange thicknesses were $0.5 \%$. The deck is overlaid with a wearing surface layer of thickness $3 / 8$ ". Several debonds were placed in between the wearing surface and the underlying FRP deck. The debonds were placed on the surface of the deck specimen, which was followed by the application of $3 / 8$ " thick wearing surface on the entire top surface. One side of the specimen BDla consisted of two debonds of sizes 2"x2" and 3"x3" and the other side BD1b consisted of two debonds of sizes 1 "x 1 " and $1 / 2$ " $\mathrm{x}^{1 / 2}$ " between the wearing surface layer and the deck. All the debonds had thicknesses of $1 / 16 "$ ".

Bridge deck BD2 was a low profile FRP deck with plan size of 24 " x12", depth of 4 " and flange thickness of 0.45 ". The top surface of the deck was coated with $3 / 8$ " thick wearing surface. Two water-filled debonds of sizes 2"x2" with thickness of approximately $1 / 16$ " and 3"x3" with thickness of 1/8", and an air-filled debond of size 2 " 2 " $\times 1 / 16$ " were placed on the surface of the deck, prior to the application of the 
wearing surface layer. Figure 4.3(b) shows the schematic view of the cross section and its dimensions.

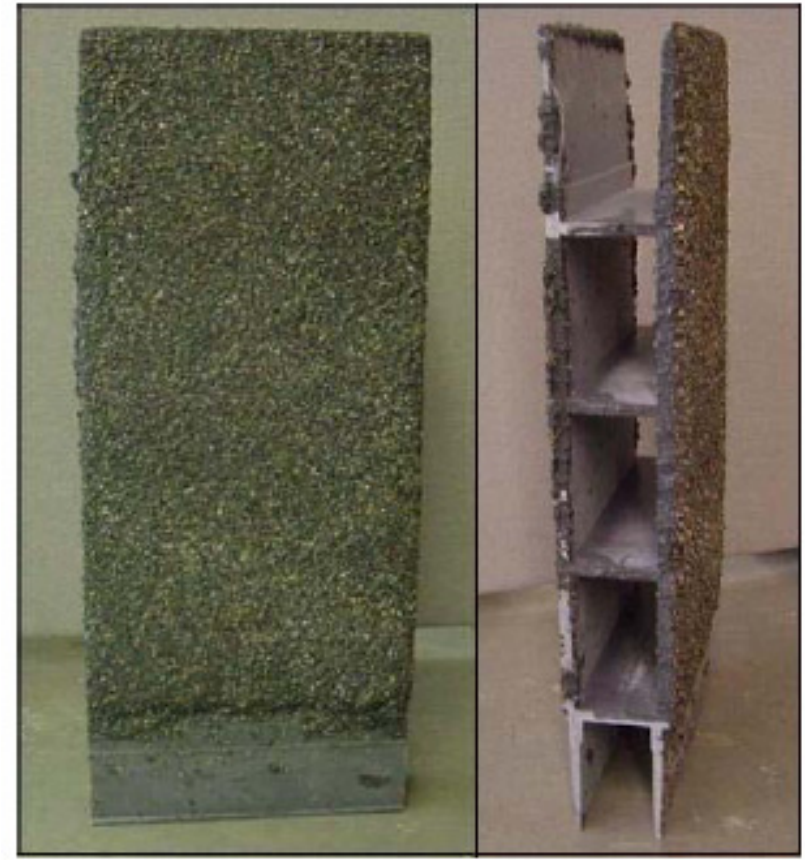

(a)

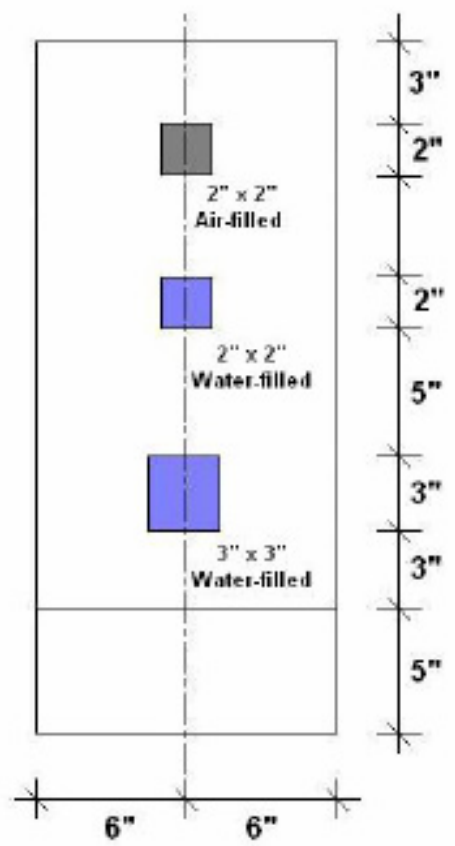

(b)

Figure 4.3: (a) Front view and cross-sectional view of the GFRP bridge deck specimen BD2 (b) schematic view of location of debonds in BD2 [10].

\subsubsection{Bridge deck specimen JD1, JD2}

Specimen JD1 was a low-profile GFRP bridge deck, with material and fiber architecture similar to the deck BD2 described in Section 4.3.1, except that it did not have the wearing surface layer. Specimen JD1 had a plan size of 15"x8" and flange thickness of 0.45 ". The thickness at the junction area, where two modules were joined was about 0.6". On one side (JD1a), a delamination of size 3"x3" in plan and 1/20" thickness was created in the middle of the flange joint area (top flange). The other side (JD1b) had two delaminations of sizes 2"x2" and 1"x1", both with thicknesses of 1/16". Neither side (JD1a or JD1b) had any wearing surface layer. Specimen JD2 had dimensions similar to specimen JD1. The difference was that it had a $3 / 8$ " thick wearing surface covering the deck flange with artificial delamination. The specimen had an artificial delamination of 3 "x3"x1/8" at the flange-flange junction. 


\subsubsection{Bridge Deck Specimen ASP1 and ASP2}

Bridge deck specimen ASP1 was a low profile deck with plan size of 24"x12". The overall depth of the deck was 4" while the flange thickness was 0.45 " and the web thickness was 0.4 ". The top surface of the deck was covered by a 2 " thick asphaltwearing surface. To determine detectability of the air-filled debonds with the infrared camera two defects of sizes 4"x4"x1/16" and 2"x2" x1/16" were embedded underneath the wearing surface. Similarly two defects of sizes 3"x3"x1/16" and 1"x1"x1/16" were embedded underneath the wearing surface to simulate air-filled debonds.

\subsubsection{Bridge Deck Specimens WJD1 WJD2 and WJD3}

Specimen WJD1 and WJD3 were low-profile GFRP composite decks with material property similar to ASP1. There was no wearing surface on these bridge deck modules. The plan size of these specimens was 24 "x 8 " with a flange thickness of 0.45 ". The average thickness of the flange-to-flange joint was 0.6". Two water-filled delaminations of sizes 2"x2" and 1"x1" were inserted in the 1/16" thick groove at the flange-flange junction on both sides. Similarly water-filled delaminations of size 3"x3" were inserted centrally on both sides (side 1 and side 2 ) of the deck joints of WJD2. Two water filled-delaminations (same sizes as in WJD1) were also inserted at the flangeflange junction on side 1 of specimen WJD3. On side 2 of specimen WJD3, a 3"x3"x1/16" water-filled delamination was inserted.

\subsection{Experimental Setup}

The experimental setup is the same as explained in Section 2.7. The diagrammatic representation of the setup is shown in Figure 2.11. Thermal images of the bridge deck were captured by a FLIR ThermaCAM S60 Infrared camera after the deck was heated or cooled. A laptop computer connected to the infrared camera was used to store the infrared images. The computer could also communicate with the infrared camera using the ThermaCAM Researcher software provided by FLIR. 


\subsubsection{FLIR ThermaCAM S60 Infrared Camera}

The FLIR ThermaCAM S60 infrared camera was used to capture infrared images of the FRP bridge decks. The ThermaCAMTM S60 infrared camera shown in Figure 4.4 is a highly sensitive lightweight (less than $4.4 \mathrm{lb}$ ) portable device with a $24^{\circ}$ lens enabled to detect radiation within the spectral range of 7.5 to 13 microns. It can detect temperature difference as small as $0.06^{\circ} \mathrm{C}$.

The different temperature ranges available for the measurement are 0 to $+500^{\circ} \mathrm{C}$ $\left(+32\right.$ to $\left.+932^{\circ} \mathrm{F}\right),-40$ to $+120^{\circ} \mathrm{C}\left(-40\right.$ to $\left.+248^{\circ} \mathrm{F}\right)$ and +350 to $1500^{\circ} \mathrm{C}\left(+662\right.$ to $\left.+2732^{\circ} \mathrm{F}\right)$. The measurements can be detected up to an accuracy of $\pm 2{ }^{\circ} \mathrm{C}$ or $\pm 2 \%$ of the temperature reading. The imaging performance for the camera has a spatial resolution of $1.3 \mathrm{mrad}$ and can record images at a frequency of $50 / 60 \mathrm{~Hz}$, non-interlaced. It is possible to capture and store images on a removable flash card.

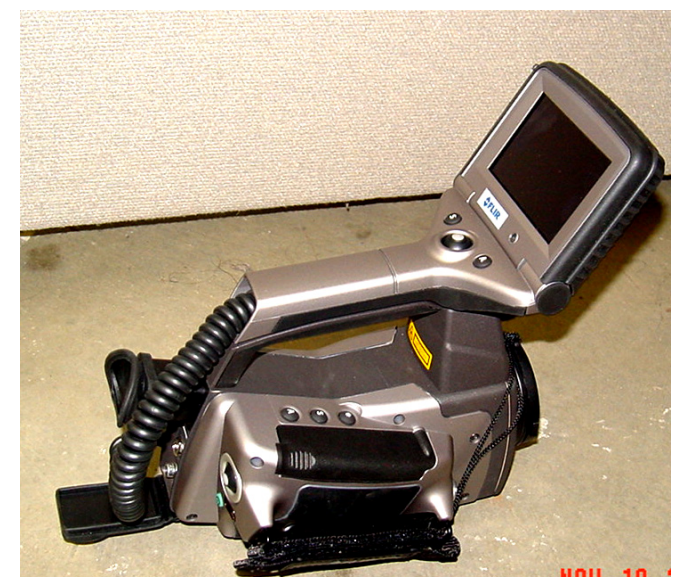

Figure 4.4: Photograph of FLIR ThermaCAM ${ }^{\mathrm{TM}} \mathrm{S60}$ infrared camera.

\subsubsection{ThermaCAM Researcher}

The infrared images can also be acquired and analyzed in a PC with the help of “ThermaCAM Researcher 2002" software. The software can be used to display live infrared images through the camera to PC interface and record the images. It can also show recorder images even when the camera is not connected. The software can be used to analyze the stored images using various built in features. Some of the features are its capability to show and draw isotherm, spot meter, line, circle, box area, polygon or user 
defined formulas and provides statistical analysis of the covered area. An example window is shown in Figure 4.5 which shows an infrared image of a bridge deck with box area and line drawn in certain areas (seen in blue color). A statistical analysis of these area performed by the software is also seen in the figure. A detailed description is found in the FLIR ThermaCAM Researcher datasheet [33].

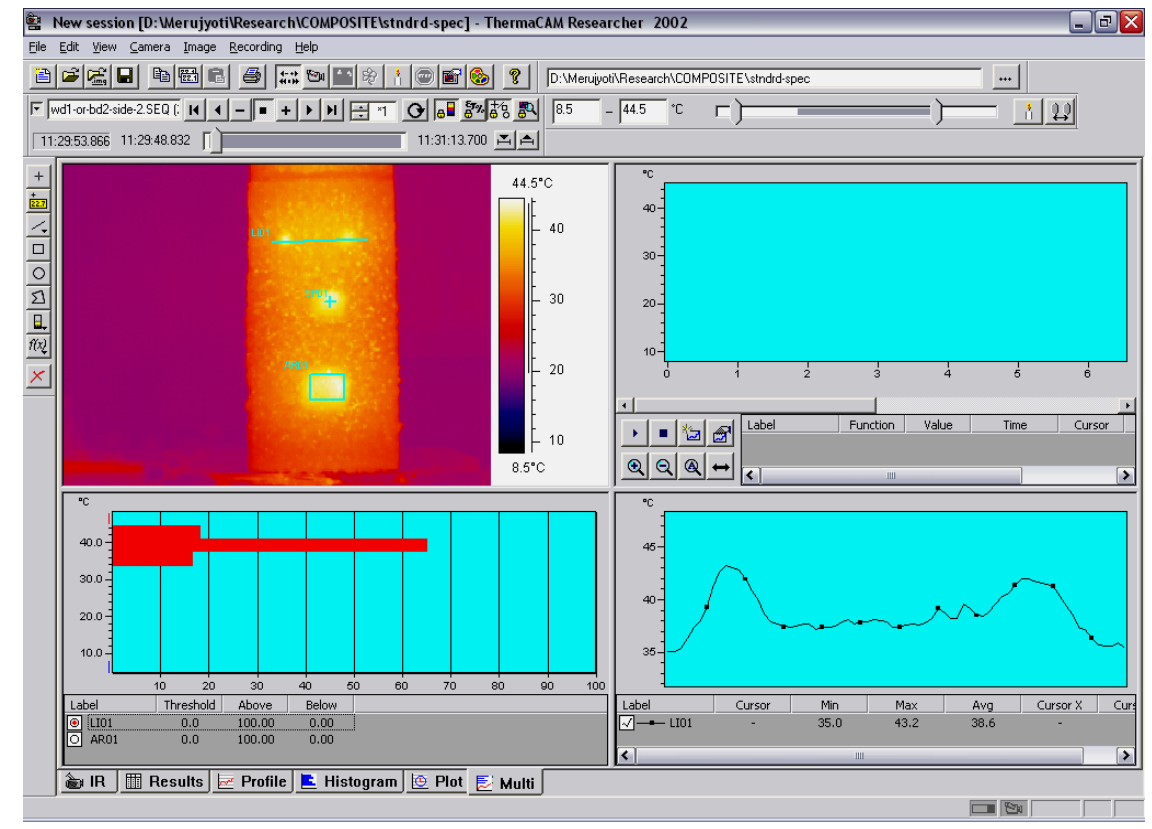

Figure 4.5: Window of a ThermaCAM Researcher 2002 showing different features [4].

\subsection{Results of Automated Defect Detection}

Infrared images of the FRP decks were captured by the infrared camera under various conditions. They were converted to JPEG format by the ThermaCAM Researcher software and given as input to the automated defect detection algorithms. A sequence of images of the same bridge deck was given as input to the infrared image mosaicking algorithms. Section 4.6 to Section 4.13 discusses the various tests and result of automated defect detection. The mosaicking approaches are compared in Section 4.14 and Section 4.15

Various tests were performed to validate the accuracy of defect detection algorithms. The algorithms developed for detection of defects in FRP bridge decks were 
discussed in Section 3.4. Infrared images were captured using the setup shown in Figure 2.11. The bridge deck under consideration is exposed to a variety of different conditions that can occur during the period of operation of a deck. The conditions tested were:

1. Detection of air-filled and water-filled defects.

2. Detection of defects with $\mathrm{CO}_{2}$ sprayed on the GFRP deck.

3. Debonds and delamination of sizes 2 "x2", 1 "x 1 " and 0.5 "x 0.5 ".

4. Effect of wearing surface (decks with and without wearing surface).

5. Effect of distance on detection accuracy (distance of deck form the camera).

6. Effect of Solar Radiation on detection accuracy.

All of the above conditions such as type of defect, size of defect, distance of infrared camera from the defect, affect the accuracy of defect detection. The results depicting the effect of these conditions on the automated defect detection algorithms are explained in detail in the next sections.

\subsection{Detection of Debonds}

\subsubsection{Test Description}

The aim of this test is to observe the accuracy of the automated defect detection algorithms for detection of debonds in FRP bridge decks. Infrared images of bridge decks with debonds of various sizes with wearing surface, were given as input to the automated defect detection algorithms.

\subsubsection{Test Results}

The results of automated defect detection of debonds are shown in Figure 4.6 and Figure 4.7. Figure 4.6(a) shows an infrared image of bridge deck BD1 side a, that has a 2"x2" defect on top and a 3"x3" defect embedded at the bottom. The two defects have been detected by the software. The 3"x3" defect was detected accurately while the size of the 2"x2" defect was detected smaller than the actual size. This may be because of uneven heating of the bridge deck. The infrared image of the side $b$ of bridge deck (BD1) 
is shown in Figure 4.6(b). It has debonds of 1 " $\mathrm{x} 1$ " and 0.5 " 0.5 " embedded below the wearing surface. The 1"x1" defect was detected accurately while the detection of the 0.5 " 0.5 " defect generated a lot of spuriously detected defect areas. The reason for this may be because the heat applied to the 0.5 " $\mathrm{x} 0.5$ " defect, may not be enough to create a distinctive temperature difference needed for accurate detection.

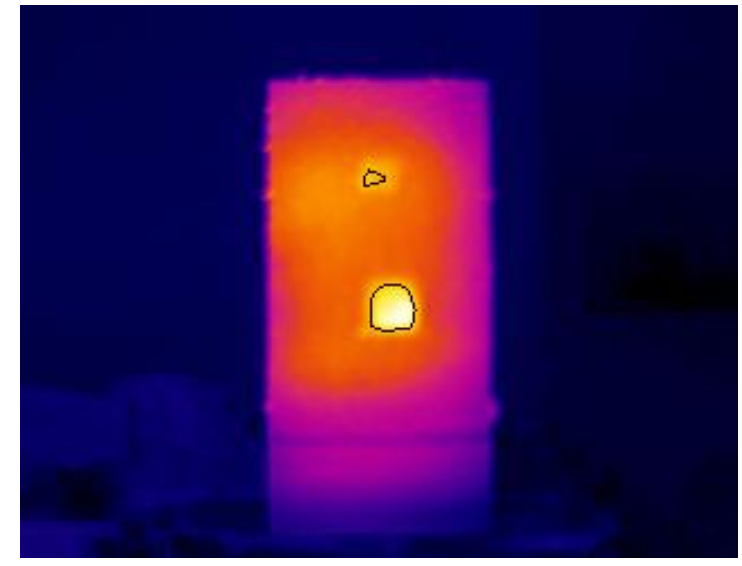

(a)

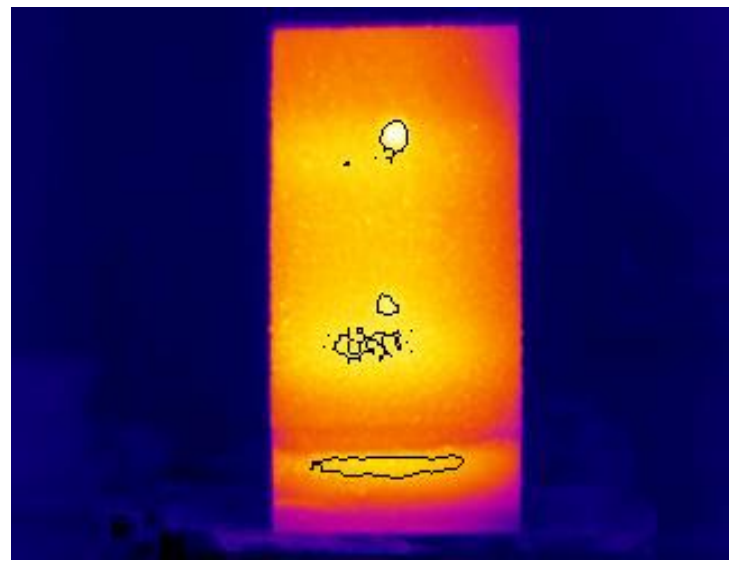

(b)

Figure 4.6: (a) Infrared image of bridge deck BD1 side A with 3"x3" and 2"x2" debonds. (b) Infrared image of bridge deck BD1 side B with 1 "x1" and 0.5 "x0.5" debonds.

Figure 4.7(a) shows the same bridge deck as seen in Figure 4.6(a) but under different heating conditions. In this case both the 3"x3" and 2"x2" defects were detected accurately but it was accompanied by a spurious defect detection. A similar case is observed for the defects seen in Figure 4.7(b). A slight change in the thermal profile of the bridge deck changes the detection results. Even though the size of the 0.5 " 0.5 " defect in this figure detected inaccurately, it should be noted that the defect may not be apparent to the human eye. The algorithm is able to locate the defect in an early stage. 


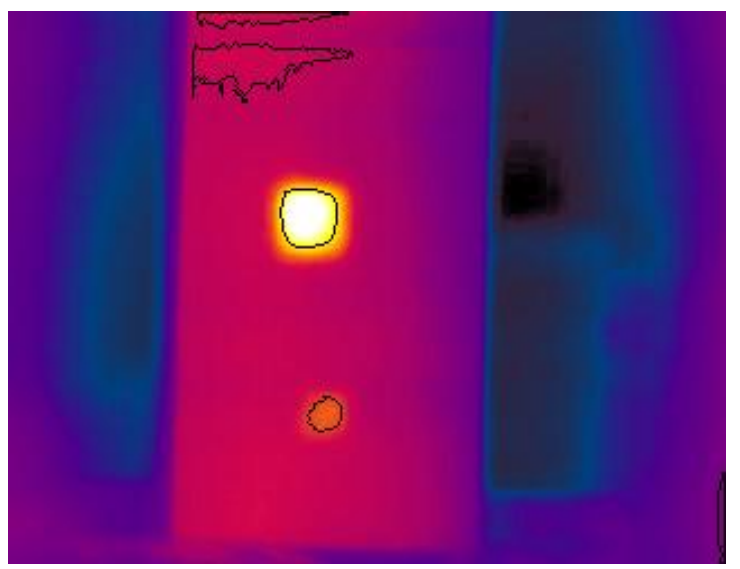

(a)

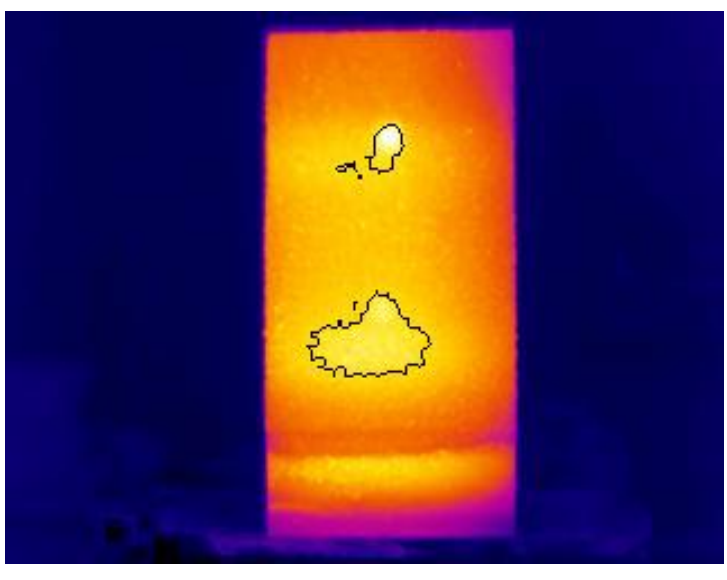

(b)

Figure 4.7: (a) Infrared image of bridge deck BD1 with 3"x3" and 2"x2" debonds under different heating condition (b) Infrared image of bridge deck BD1 with 1 "x1" and 0.5 "x0.5" debonds under different heating conditions.

\subsubsection{Test Conclusion}

The automated defect detection system is able to accurately detect defects of size 1 "x1" or more. The defects of sizes less than 1"x1" may not be detected accurately. The accuracy of defect detection will depend upon the amount of heat supplied during the image capture phase as well as on the size of the defect. Another important observation was that enough heating of the bridge deck surface will ensure a high defect detection accuracy.

\subsection{Detection of Delaminations}

\subsubsection{Test Description}

The aim of this test is to observe the accuracy of the automated defect detection

algorithms for detection of delaminations. Infrared images of bridge decks with delamination of various sizes, with and without wearing surface were given as input to the automated defect detection algorithms.

\subsubsection{Test Results}

The tests were conducted on FRP bridge decks JD1 and JD2. Figure 4.8(a) shows an infrared image of bridge deck JD2 with a 3"x3" defect. The image clearly shows the 
defect accurately recognized by the algorithms. However, heat had to be applied for approximately 5 minutes to generate a distinguishable temperature difference required for defect detection. This is because the application of the wearing surface increases the depth of the defect from the surface. Bridge deck JD1 on the other hand, had defects of sizes 1"x1" and 2"x2". There was no wearing surface on the deck. These defects (seen in the center of Figure 4.8(b)) are accurately segregated by the algorithm. The estimated size of 1 "x 1 " defect is observed to be slightly more than the actual one.

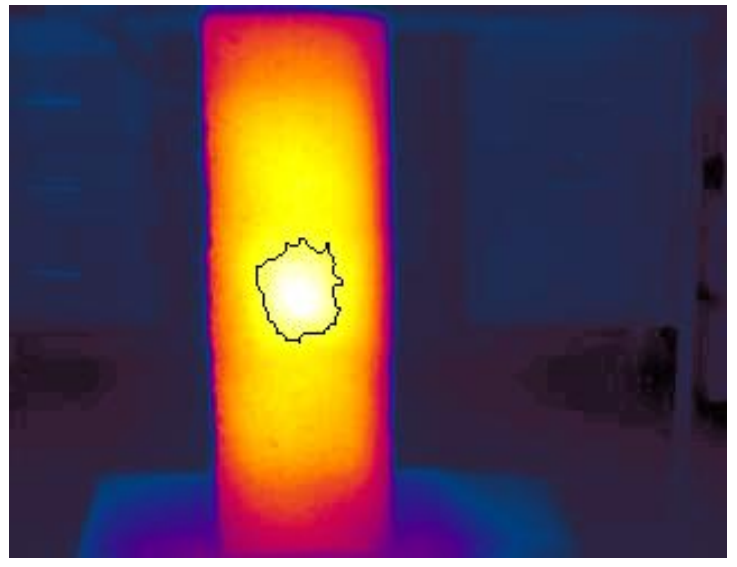

(a)

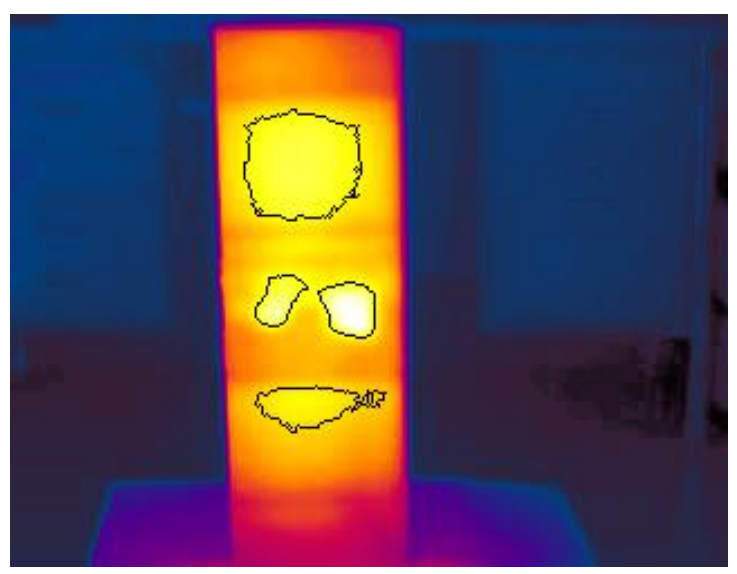

(b)

Figure 4.8: (a) Infrared image of bridge deck JD2 having 3"x3" delamination with wearing surface (b) Infrared image of bridge deck JD1 having 1"x1" and 2"x2" delamination without wearing surface.

Also spurious defects were estimated by the algorithm at the top and bottom in Figure 4.8(b). The reason for this may be because of the way in which delaminations are created. Delaminations are inserted while joining two bridge decks with each other. This is shown in Figure 4.9 where the two decks are seen to have a joint at the center. There is an area of overlap between the two decks at the joint. Because of this, the place where the joint ends at the top and bottom of the deck dissipates more heat. Hence these areas are falsely characterized as defect areas by the automated defect detection algorithm. 


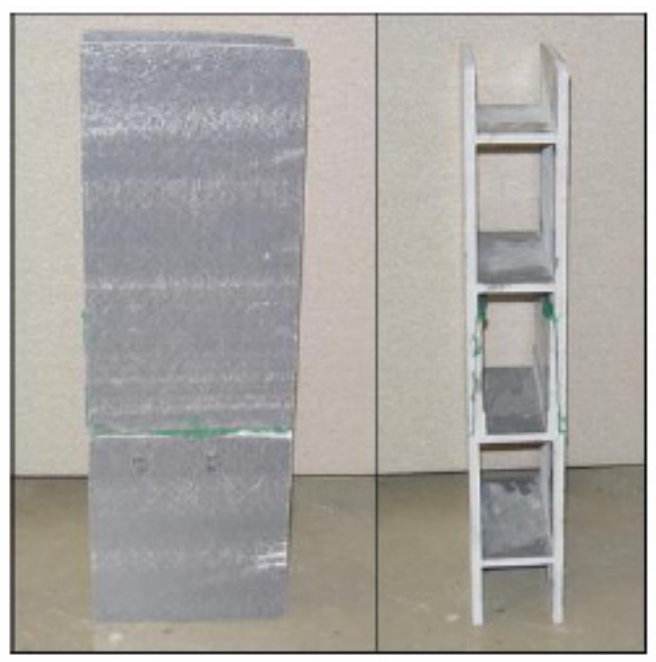

Figure 4.9: Front view and cross-sectional view of the FRP bridge deck JD1 w/o wearing surface [10].

\subsubsection{Test Conclusion}

The algorithm was able to detect delaminations in FRP bridge decks. The amount of heat required to generate a temperature difference between the defect and the defect free area is more in the case of FRP decks with delaminations. This is because the greater the depth of the delamination from the top surface, the more difficult it is to detect it. Research by Maldague [9] has shown that as the contrast between the defective and defect-free areas is inversely proportional to the cube of the depth. Hence it was difficult to detect of size 1 "x 1 " accurately.

\subsection{Detection of Air Filled and Water Filled Defects}

\subsubsection{Test Description}

Infrared images from various bridge decks were used for this test. The aim was to find out whether the algorithms are able to detect the two main types of defects in FRP bridge decks. i.e. air filled defects and water filled defects. Infrared images from different bridge deck specimens with air filled and water filled debonds and delaminations were used for this test. They are:

1. BD1 with 3"x3" and 2"x2" air filled debond.

2. BD2 with 3"x3" air filled debond 3"x3" and 2"x2" water filled debond.

3. JD2 with 3"x3" air filled delamination. 


\subsubsection{Test Results}

Figure 4.10 shows the results of defect detection for four different cases. The defects in each image are marked by a black outline. The image in Figure 4.10(a) is that of bridge deck with 3"x3" air filled delamination. The defect is detected accurately. The image in Figure 4.10(b) shows the 3"x3" and 2"x2" defect marked accurately. The size of the 2" 2 "defect is not detected accurately. It is outlined as a defect of smaller size. This may be because of uneven heating of the defect.

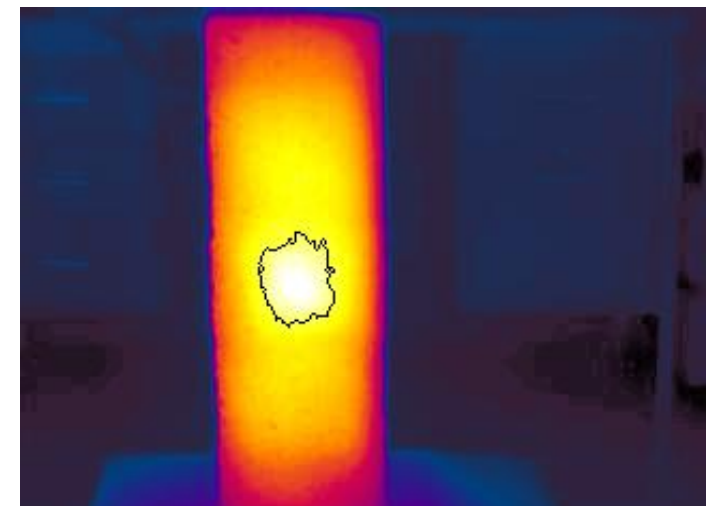

(a)

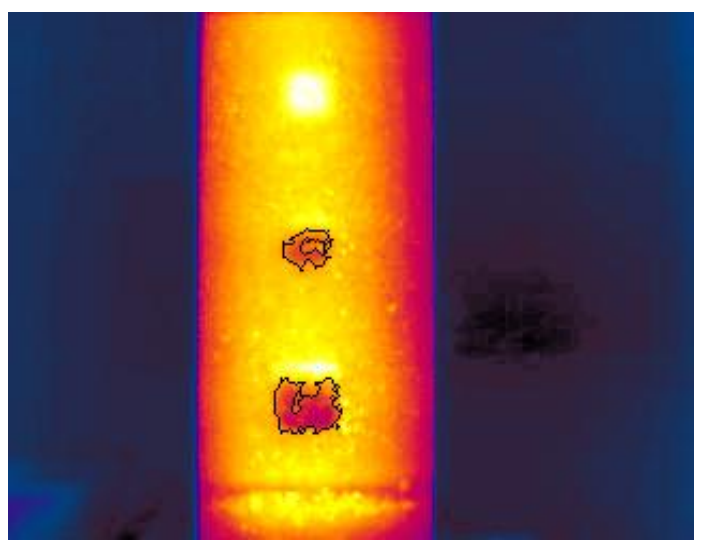

(c)

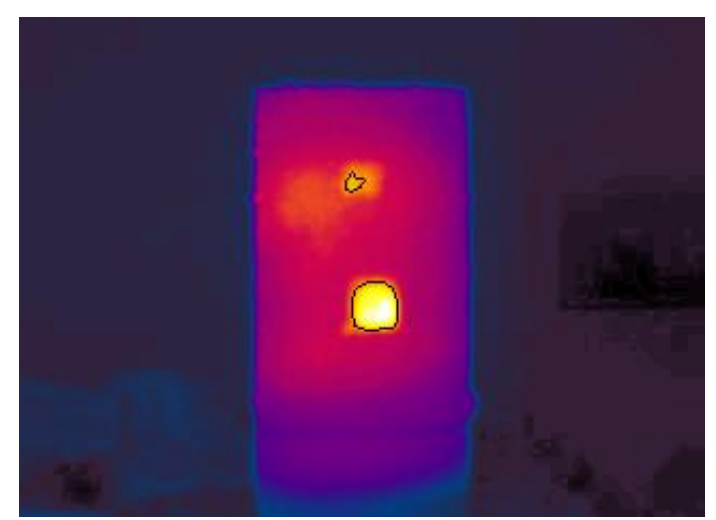

(b)

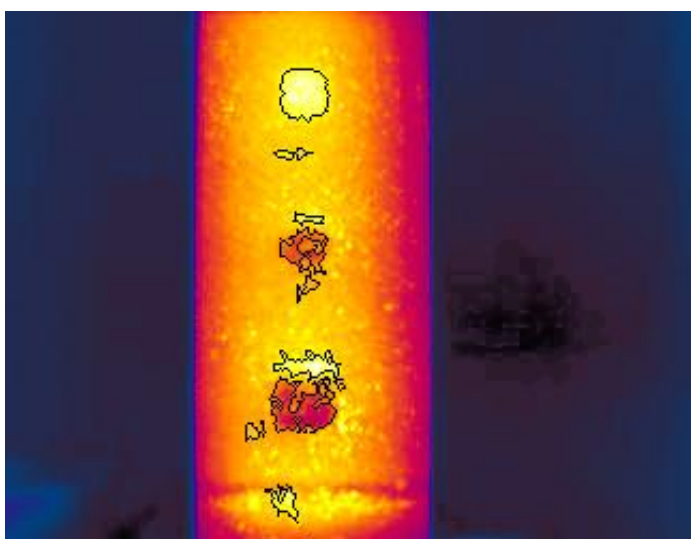

(d)

Figure 4.10: (a) Infrared image of specimen JD2 with 3"x3" air filled delamination. (b) Infrared image of specimen BD1 with 3" x3" and 2" x2" air filled debond. (c) Infrared image of specimen BD2 with 3"x3" and 2"x2" water filled and 3"x3" air filled debonds. Algorithm set to detect water filled debonds. (d) BD2 with algorithm set to detect water and air filled debonds.

Figure 4.10(c) shows bridge deck BD2 with 3"x3" and 2"x2" water filled debonds and 3"x3" air filled debonds. In this case the algorithm is asked to locate only water filled 
defects. The bottom two defects were accurately detected as water filled defects and the air filled defect on top was discarded. The same bridge deck image is given as input and the algorithm parameters are set to detect both, the air filled and the water filled defects. As seen in Figure 4.10(d) the upper air filled defect has been detected with the lower water filled defects accurately.

\subsubsection{Test Conclusion}

Infrared images of specimens having debonds and delaminations with either air filled or water filled defects were used during the test. The algorithm is able to detect both air filled and water filled defects of various sizes from these specimens. Accuracy of automated defect detection depends on many factors such as amount of heat applied, depth of the defect from the surface of the deck etc. Accurate automated detection of water filled defects may be difficult as enough temperature difference may not be created. Also, non-defect areas may be falsely detected as water filled defects. This is because water filled defects create an inverse temperature difference. i.e. the temperature to the defect area is less in comparison with the surrounding area. The presence of pebbles may create such a temperature difference causing false detection.

\subsection{Effect of Distance on Defect Detection}

\subsubsection{Test Description}

The aim of this test was to find out the effect of varying the distance between the camera and the bridge deck on the detection accuracy. In this test infrared image of the specimen BD1 that has 3"x3" and 2"x2" debonds and specimen JD1 with 1"x1" and 2"x2" delamination are acquired at varying distances of 3, 4, 5 and 6 meters. These images are given as input to the automated defect detection algorithm.

\subsubsection{Test Results}

The images in Figure 4.11(a)(b) are infrared images with the defected defects, of bridge deck BD1 at a distance of 3 and 4 meters respectively. The deck has a 3"x3" debond at the top and a 2"x2" debond at the bottom. The image in Figure 4.11(a) shows 
that both the 3"x3" and the 2"x2" defects are detected accurately. As the distance between the deck and the camera increases to 4 meters the size of the automatically detected 3"x3" defect reduces. On the other hand, the size of the 2"x2" defect increases and is almost comparable to the size of the 3" 3 " defect is clearly seen in Figure 4.11(b).

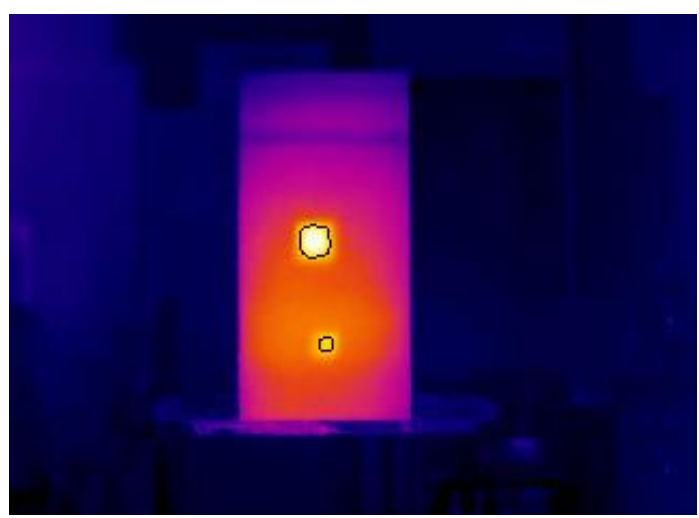

(a)

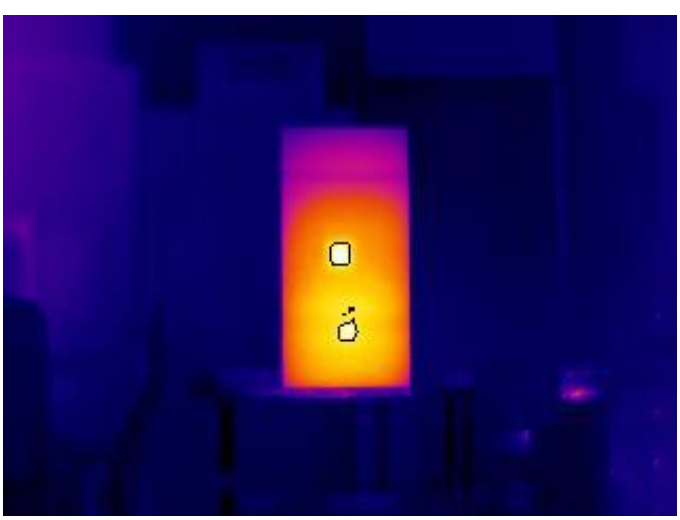

(b)

Figure 4.11: Infrared images of specimen BD1a with debonds of sizes 2"x2" and 3"x3" detected at a distance of (a) $3 \mathrm{~m}$ (b) $4 \mathrm{~m}$.

Ideally, as the distance increases from 4 to 5 and 6 meters, the size of the detected defects should also decrease. Contradictory results are seen from Figure 4.12(a)(b) which depict the size of the defects detected in infrared image of bridge deck specimen BD1 at a distance of 5 and 6 meters respectively. The infrared image in Figure 4.12(a) shows that the size detected by the automated algorithm, of the 2"x2" defect has decreased to a tiny dot as desirable. The detected size of the 3 " 33 " defect on top has increased with respect to the size of the detected defects in Figure 4.11(b). An extension of the same phenomenon is seen in Figure 4.12(b) where only the 3"x3" defect is detected. The detected size of the 3"x3" defect is larger than that observed in Figure 4.11(b). The 2"x2" defect is not at all detected in this figure. 


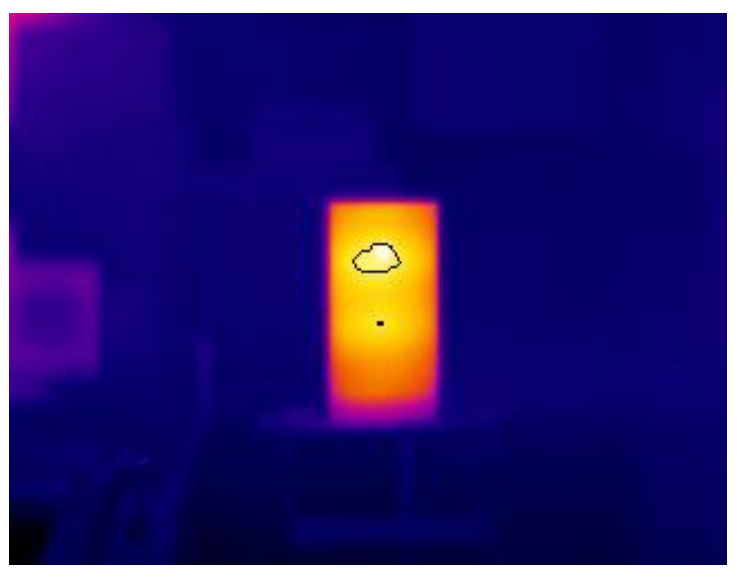

(a)

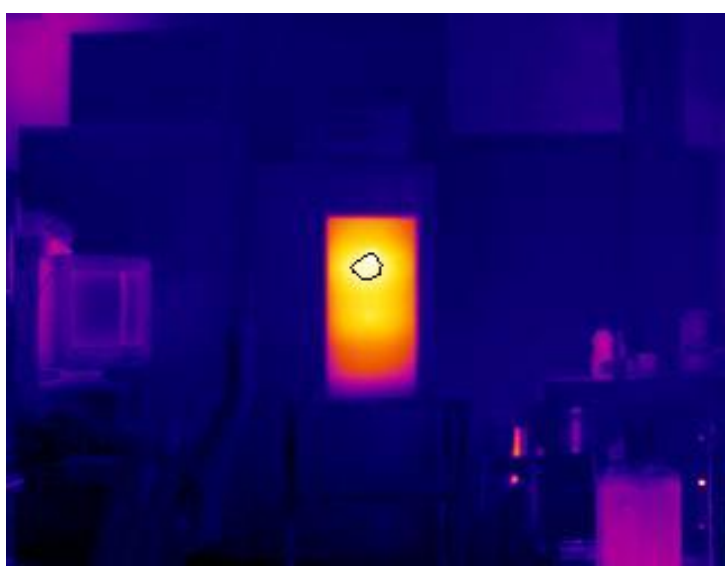

(b)

Figure 4.12: Infrared images of specimen BD1a with debonds of sizes 2"x2" and 3"x3" detected at a distance of (a) $5 \mathrm{~m} \mathrm{(b)} 6 \mathrm{~m}$.

A similar result i.e. an increase in detected size of the defect as the distance increases from 3 meters to 4 and 5 meters, is observed from Figure 4.13(a)(b)(c). The figures show infrared images with the detected defects of specimen JD1 side $b$ that has a 1"x1" defect and a 2"x2" defect. The image in Figure 4.13(a) shows that both the 1"x1" and the 2"x2" defects are detected with almost the same size and a spurious defect is also detected. As the distance between the camera and the deck increases to 4 meters in Figure 4.13(b) the size of the detected defects should decrease. Instead both the defects are now recognized as a bigger single defect (Figure 4.13(b)). When the distance increases to 5 meters, the size of the detected defects further increases.

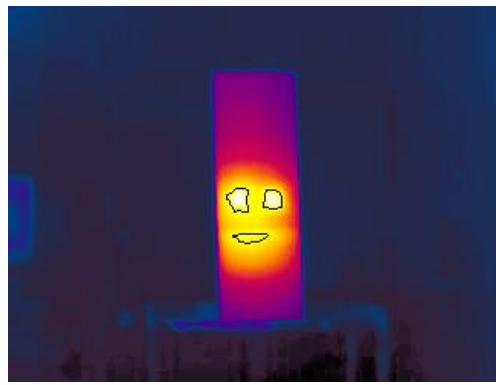

(a)

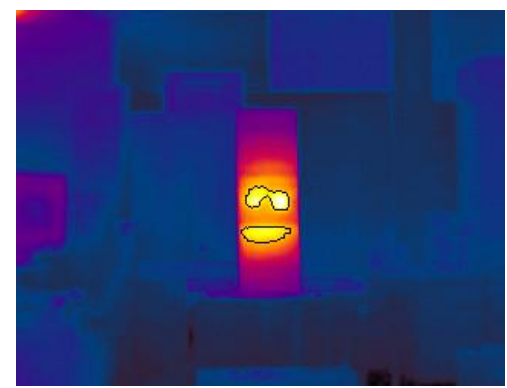

(b)

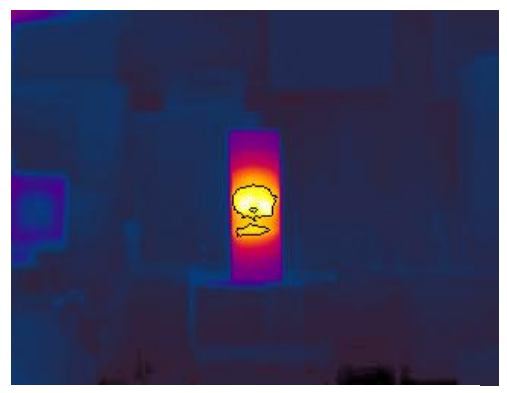

(c)

Figure 4.13: (a) Infrared image of specimen JD1 having 1"x1" and 2"x2" delaminations at a distance of 3 meters with the detected defects. (b) Infrared image of specimen JD1 having 1"x1" and 2"x2" delaminations at a distance of 4 meters with the detected defects. (c) Infrared image of specimen JD1 having 1"x1" and 2"x2" delaminations at a distance of 5 meters with the detected defects. 


\subsubsection{Test Conclusion}

It is clearly seen from the results shown in Figure 4.12 and Figure 4.13 that the accuracy of the defect detection algorithms is a function of the distance between the camera and the bridge deck. i.e. for a given size of a defect, say 3"x3" defect, the accuracy of detection and estimation of correct defect size will be high when the distance between the camera and the deck is 3 meters. As the distance between the camera and the deck increases the detection accuracy decreases. The detection accuracy is also dependent on the size of the defect. i.e. for a given distance of say 4 meters a 3"x3" defect will be detected accurately in comparison to a 2 "x2" or a 1 "x 1 " defect.

The reason for the decrease in accuracy is because the number of pixels in the image that represent the defect area reduces as the distance between the camera and the deck increases. The number of pixels that represent the 3"x3" defect in Figure 4.12(a) is $324(18 \times 18)$ at a distance of 3 meters. This number reduces to 144 (12"x12) at a distance of 4 meters and 36(6x6) at a distance of 5 meters. Accurate detection is obtained at a 3 meter distance (324 pixels in defect area) and a 4 meter distance (144 pixels in defect area). The accuracy decreases drastically at the 5 meter distance (36 pixels $n$ defect area). Hence it can be concluded that a minimum of 100 pixels should represent the defect area for accurate detection of defects.

\subsection{Defect Detection under Solar Radiation}

\subsubsection{Test Description}

Solar radiation is a natural source of heat. It can be used as a passive source to heat the bridges in working conditions. In this test the FRP bridge decks were exposed to solar radiation from morning (9am) till evening $(6 \mathrm{pm})$. Infrared images of the bridge decks exposed to solar radiation were captured at regular intervals of 30 minutes $(1 / 2$ hour). These images were then given as input to the automated defect detection algorithm. 


\subsubsection{Test Results}

Figure 4.14(a)(b)(c) depict an infrared image of bridge deck BD1 side a exposed to solar radiation, that has a 3"x3" defect. The image in Figure 4.14(a) is an infrared image taken at 9:30am in the morning when Sun is on the left hand side of the deck. Hence there is an uneven heating on that causing defects to be falsely estimated on the left side (Figure 4.14(a)). The second image in Figure 4.14(b) is taken at 2pm when Sun is almost on top of the deck and the deck has been exposed to solar radiation for approximately 5 hours. The image in Figure 4.14(b) shows that the automated defect detection algorithm is accurately able to pin point the location and size of the defect area.

At $5 \mathrm{pm}$, the sun is on the right hand side of the deck shown in Figure 4.14(c). Hence some uneven heating of that side took place. Therefore along with the detection of the 3"x3" defect at the center, spurious detects are detected at the right edge of the bridge deck.

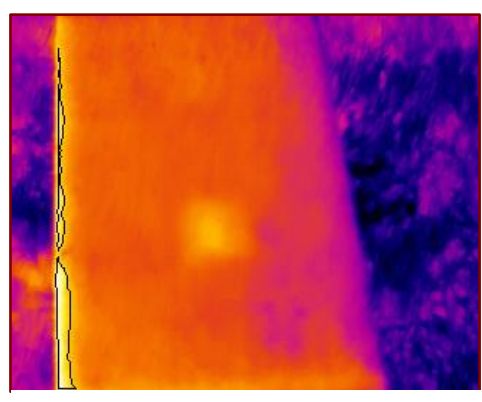

(a)

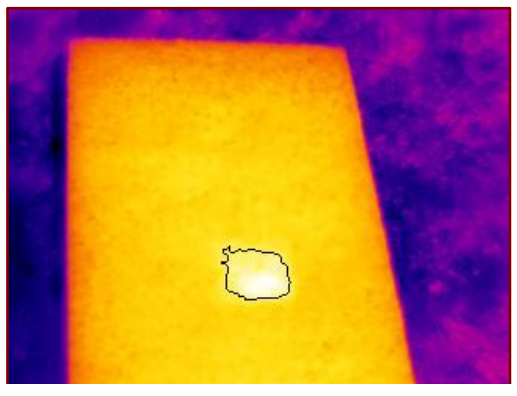

(b)

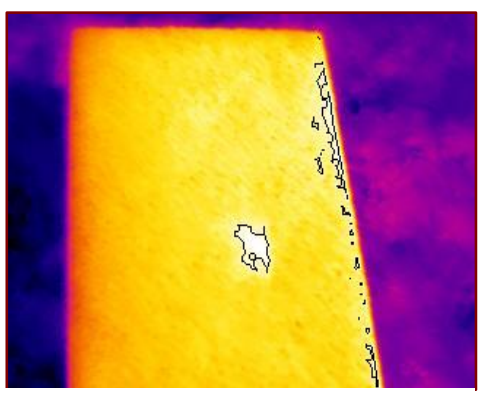

(c)

Figure 4.14: (a) Infrared image of specimen BD1 with 3"x3" debonds at 9am, with the detected defects. (b) Infrared image of specimen BD1 with 3"x3" debonds at 2pm, with the detected defects.

(c) Infrared image of specimen BD1 with 3"x3" debonds at 4pm, with the detected defects.

A similar case is seen in Figure 4.15(a)(b)(c). The figures show a solar heated infrared image of bridge deck specimen JD1 that has a delamination of 3"x3". The images in Figure 4.15 (a)(b)(c) are taken at 9:30pm, 2:30pm and 4:30pm respectively. They follow the same pattern as seen in Figure 4.14(a)(b)(c). i.e. reflection on the left edge in the image taken at 9:30am, accurate detection in the image taken at 2:30pm and reflections on the right edge in the image taken at 4:30pm. 


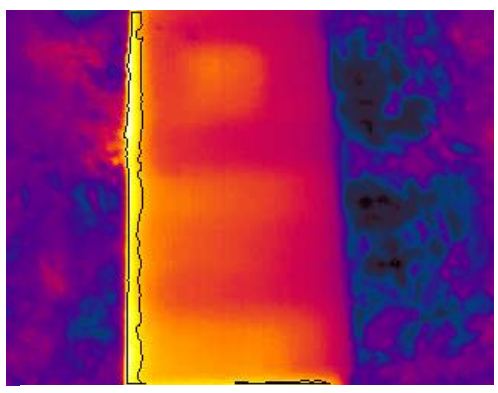

(a)

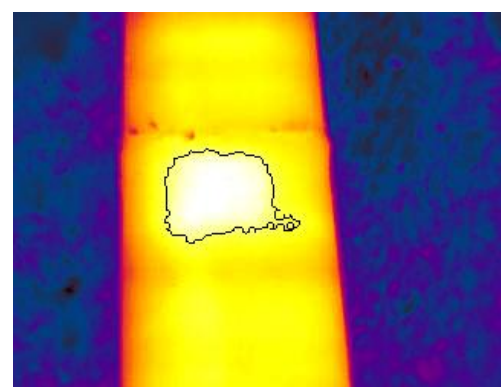

(b)

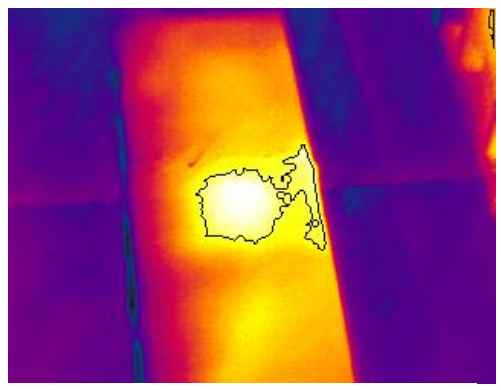

(c)

Figure 4.15:(a) Infrared image of specimen JD1 with 3" x3" delamination at 9:30am, with the detected defects. (b) Infrared image of specimen JD1 with 3" 3 " delamination at 2:30pm, with the detected defects. (c) Infrared image of specimen JD1 with 3" $x 3$ " delamination at 4:30pm, with the detected defects.

\subsubsection{Test Conclusion}

From the images in Figure 4.14 and Figure 4.15 it can bee seen that solar radiation is an uneven heat source. When under solar radiation, it takes time for the bridge decks to heat to an extent that will cause a temperature difference between the defect and the defect free area. The most appropriate time for performing infrared thermography with high accuracy is between $12: 00 \mathrm{pm}$ to $3: 00 \mathrm{pm}$. There can be detection errors in infrared images of bridge decks under solar radiation. They may be due to uneven heating of the deck by the sun or because of the presence of objects such as pebbles between the deck and solar radiation.

\subsection{Defect Detection with Cooling Sources}

\subsubsection{Test Description}

The presence of air filled defect creates a high temperature area in the bridge deck. The temperature differential between the defect area and the non-defect area may not be large enough to create an image with good contrast in which defects can be easily detected. Hence a method of suddenly cooling the heated FRP deck using liquid carbon dioxide was tested. The application of liquid carbon dioxide cools the defect areas faster creating a negative temperature differential that can be used for accurate defect detection. A simple way to cool the heated FRP deck by pouring water over the deck was also 
tested. Infrared images of FRP bridge decks were cooled using liquid carbon dioxide or water and given as input to the algorithm for defect detection in this test.

\subsubsection{Test Results}

In the first part of the test infrared image of an FRP bridge deck cooled using liquid carbon dioxide was given as input to the algorithm. The image along with the detected results of automated defect detection is shown in Figure 4.16(a). The result clearly shows the accurate detection of the 2"x2" and 3"x3" debonds in the bridge deck.

The contrast in the image is not as clear as in a thermal image of an FRP bridge deck where the defects are at a higher temperature than the surrounding area. Hence the colors of the image are inverted and it is again tested for defect detection. The liquid $\mathrm{CO}_{2}$ image with an inverted color scale along with the results of automated defect detection is shown in Figure 4.16(b). Here the defects are clearly seen and recognized by the algorithm.

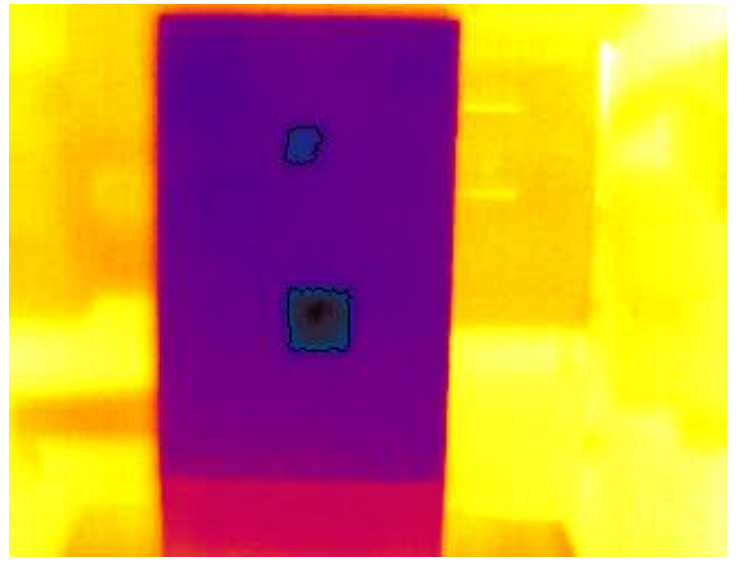

(a)

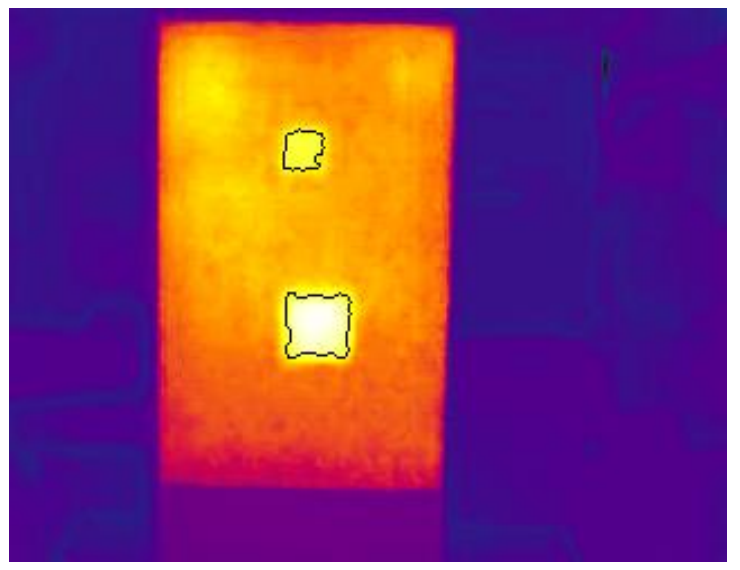

(b)

Figure 4.16: (a) Infrared image of specimen BD1a with debonds of size 2"x2" and 3"x3", cooled using liquid $\mathrm{CO}_{2}$ with defect detection results. (b) Infrared image of (a) with an inverted color scale for the temperature with defect detection results.

The other type of cooling source used was water. Since the use of liquid $\mathrm{CO}_{2}$ may not be possible on a large scale, using water as a cooling source represents a feasible alternative. The image in Figure 4.17(a) is of bridge deck specimen Jd1 side b that has a 
1 "x1" and 2"x2" delamination. Solar radiation is used as a passive heat source to heat the bridge deck specimen. Once the specimen is heated, water is sprayed on top of the deck as a method of cooling the deck and an infrared image is captured. The image with the detected defects is shown in Figure 4.17(a). It is clearly seen that the human eye may not be able to clearly pin point the location of the defects because their temperature is near the atmospheric temperature. The algorithm, on the other hand is able to locate the defects correctly. Both the 1 "x 1 " and the 2"x2" defects are correctly located and the shape is estimated with reasonable accuracy. A similar case is observed in Figure 4.17(b) that depicts an infrared image of bridge deck specimen BD1 side a with a 3"x3" defect. The algorithm is able to defect the defect. There are some spurious defect detections mainly because the contrast between the bridge deck area and the surrounding atmosphere is not as much as seen in Figure 4.17(a).

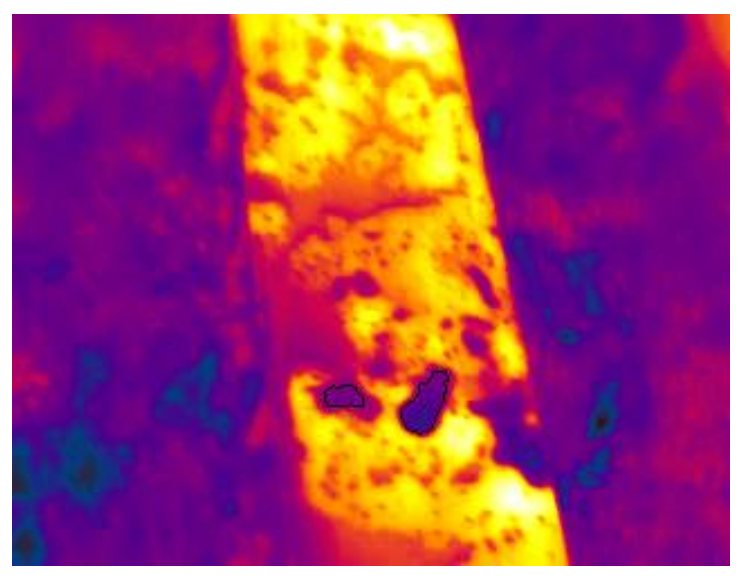

(a)

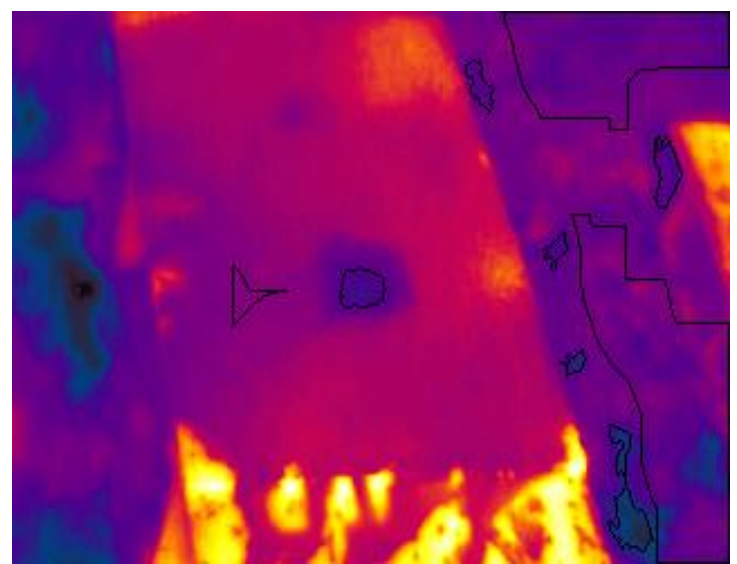

(b)

Figure 4.17: (a) Infrared image of specimen JD1b with 1"x1" and 2" 2 " delaminations, kept in solar radiation and cooled with water. (b) Infrared image of specimen BD1a with 3"x3" debonds, kept in solar radiation and cooled with water.

\subsubsection{Test Conclusion}

The use of cooling sources to increase the visualization contrast or generate a higher temperature difference between the defect and defect free area was investigated. It was found that both by using liquid $\mathrm{CO}_{2}$ and water such a situation can be achieved. The use of liquid $\mathrm{CO}_{2}$ results in an infrared image with a high contrast and temperature 
differential between the defect and defect free area. This results in accurate defect detection. The use of water on the other hand, generates an image with lots of spurious areas that look similar to defect areas. Also the contrast between the bridge deck area and the surrounding atmosphere may not be enough as seen in Figure 4.17(b). Hence some spurious defects can be detected along with the actual detection of defect as the results shown in Figure 4.17(b).

\subsection{Defect Detection on Infrared Images of Field Tests}

\subsubsection{Test Description}

The aim of this test was to evaluate the accuracy of the algorithm in detecting defects in an FRP bridge deck in operation. Field tests were conducted in the research by $[4,10]$. The FRP bridge decks are susceptible to subsurface defects, which can adversely affect their load carrying capacity. Therefore, periodic monitoring of the bridge components is necessary for timely detection of subsurface defects such as debonds and delaminations. The infrared thermography field tests conducted on the La Chein FRP (Figure 4.18) bridge deck located in Monroe County, near Peterstown in West Virginia. Details about the deck construction, wearing surface etc are given in [10].

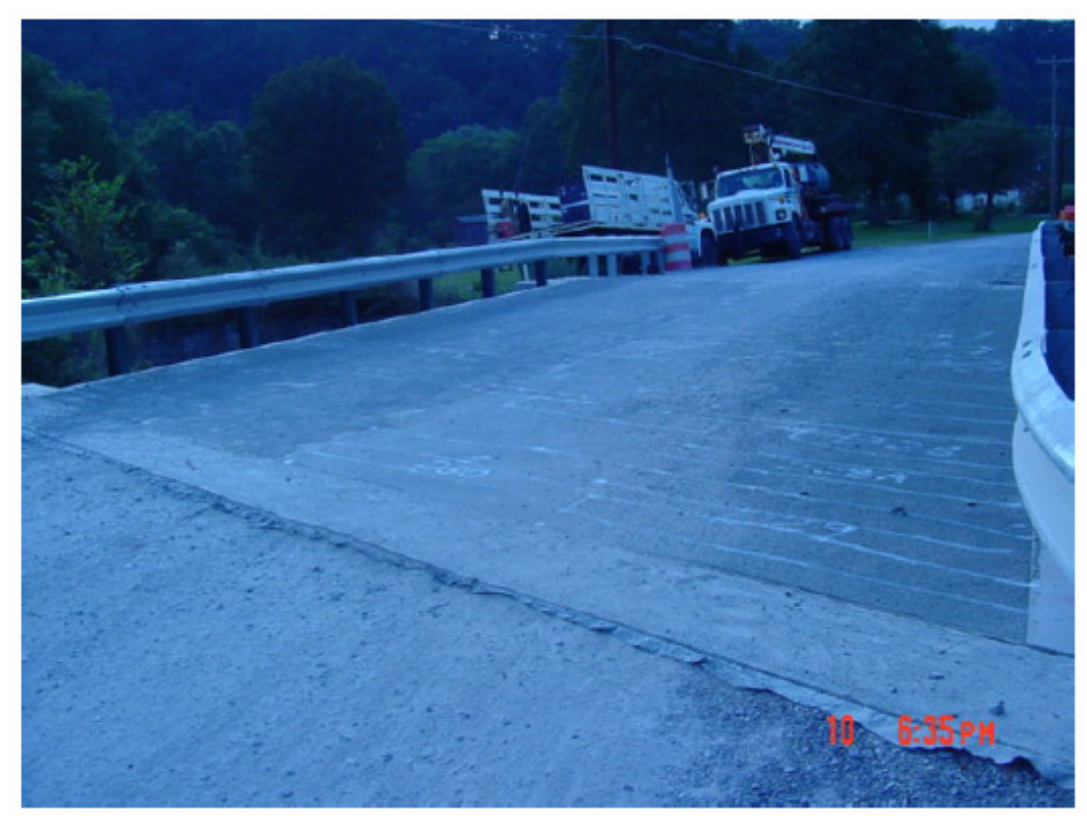

Figure 4.18: Photograph of La Chein FRP bridge [10]. 
The bridge deck was heated prior to capturing infrared images using the ThermaCAM S60 infrared camera. Solar radiation was used as a passive heating source. The deck was divided into 32 grids [10] as shown in Figure 4.19. Images were taken at around noon after the deck was heated. These images are given as input to the automated defect detection algorithm. Tap testing was performed on the entire bridge deck and the results were compared with the results from infrared thermography.

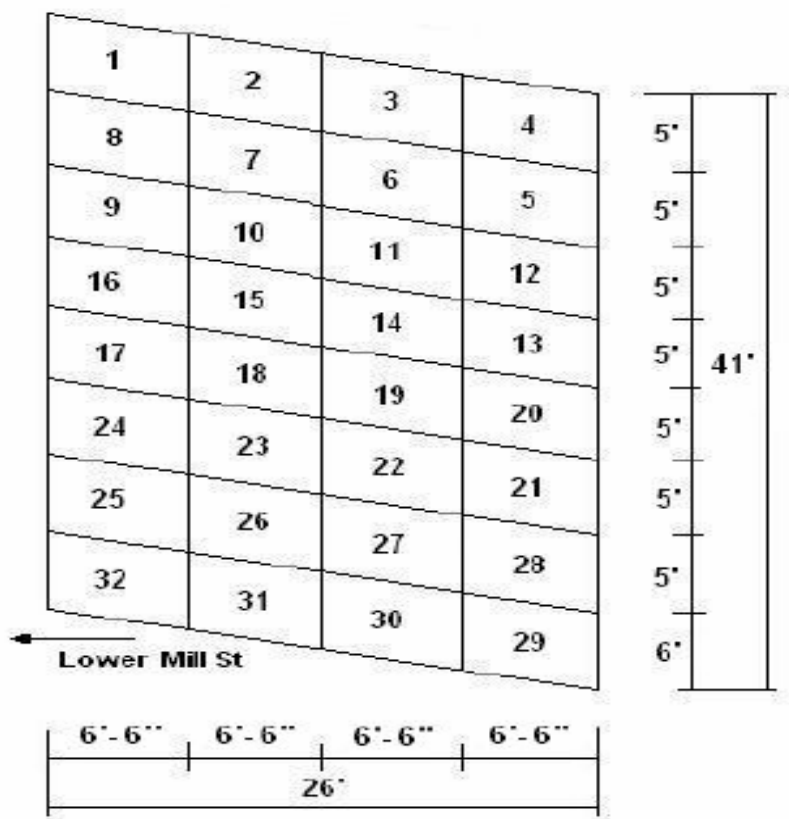

Figure 4.19: Schematic view of the grids [10].

\subsubsection{Test Results}

Some of the results of automated defect detection on infrared images of La Chein FRP bridge deck are shown in Figure 4.20, Figure 4.21, Figure 4.22 and Figure 4.23. A photograph of grid 15 along with the detected defect is shown in Figure 4.20(a)(b). The algorithm is accurately able to detect the debond in the FRP bridge deck. 


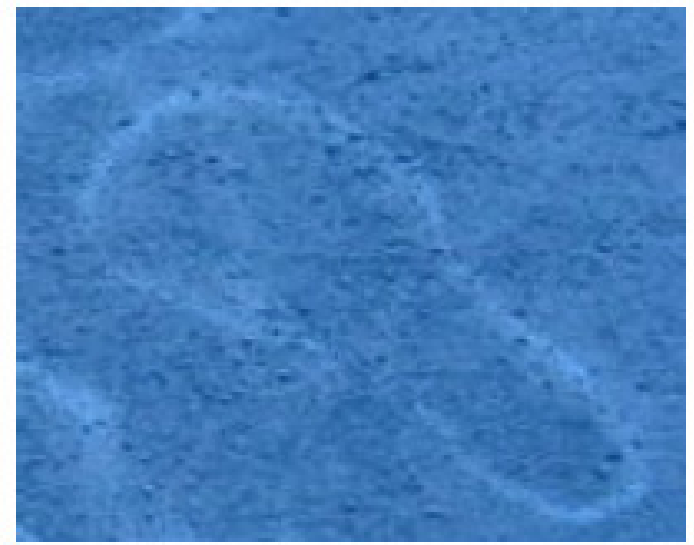

(a)

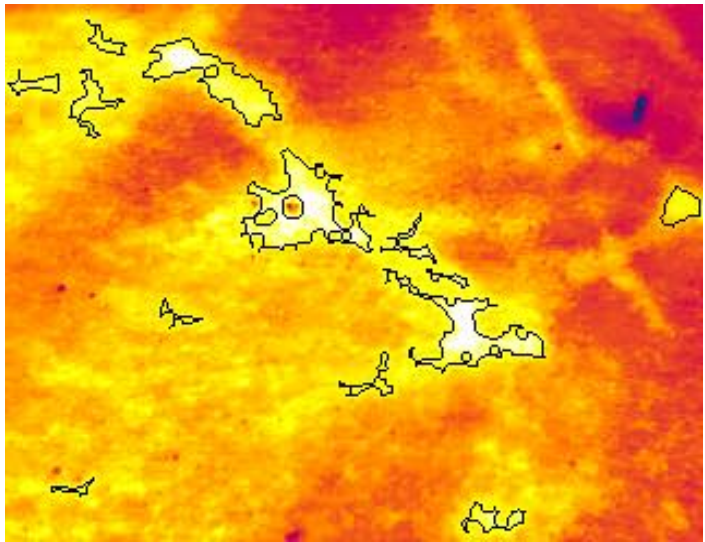

(b)

Figure 4.20: (a) Photograph of grid 15 (b) Infrared image with detected defects of debonds in grid 15.

Similarly infrared images of grid 4 along with the detected defects are shown in Figure 4.21(a). There are some pebbles, gravel etc. on the surface of the deck that results in irregular infrared images as seen in Figure 4.21(b). The algorithm however, is able to detect the defects accurately.

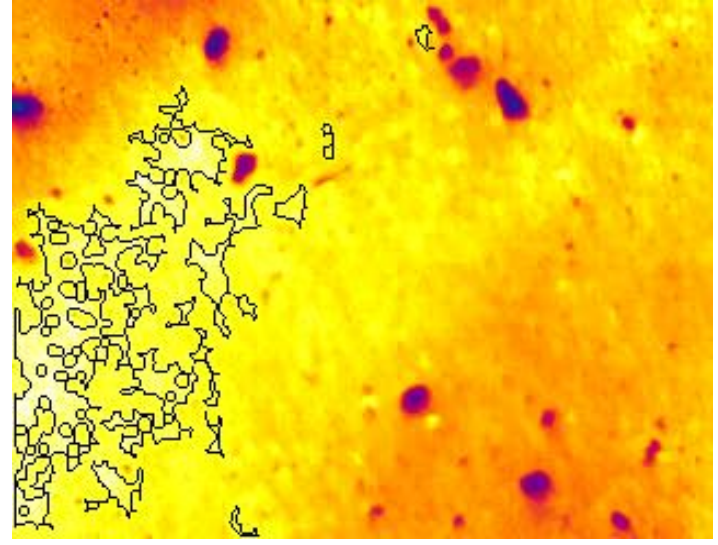

(a)

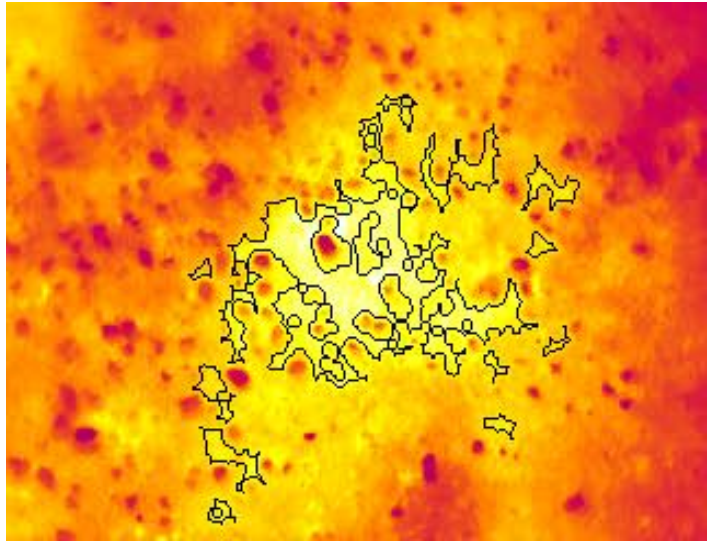

(b)

Figure 4.21: (a)(b): Infrared image of a two debonds in grid 4 respectively.

In some cases the algorithm falsely delineates some areas in the infrared image as defects. An example is shown in Figure 4.22(b) that depicts an infrared image of the FRP bridge deck with some area of a guard rail (Figure 4.22(a)). The guard rail acts a barrier 
between the sun and the bridge deck. Hence a portion of the bridge deck is not exposed to solar heating. This causes a low temperature area as seen in Figure 4.22(b). This irregular heating results in false detection of defects in Figure 4.22(b).

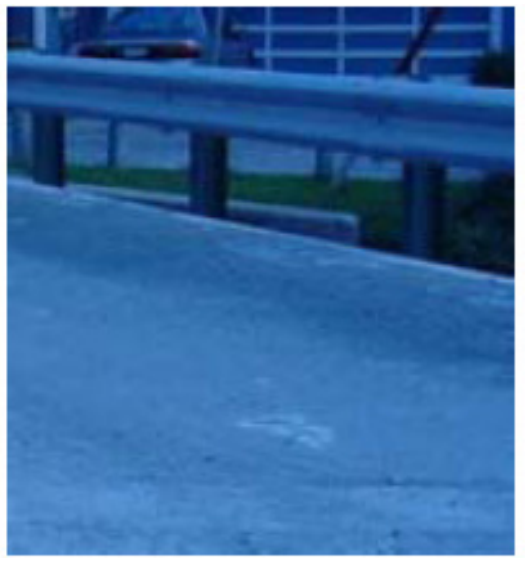

(a)

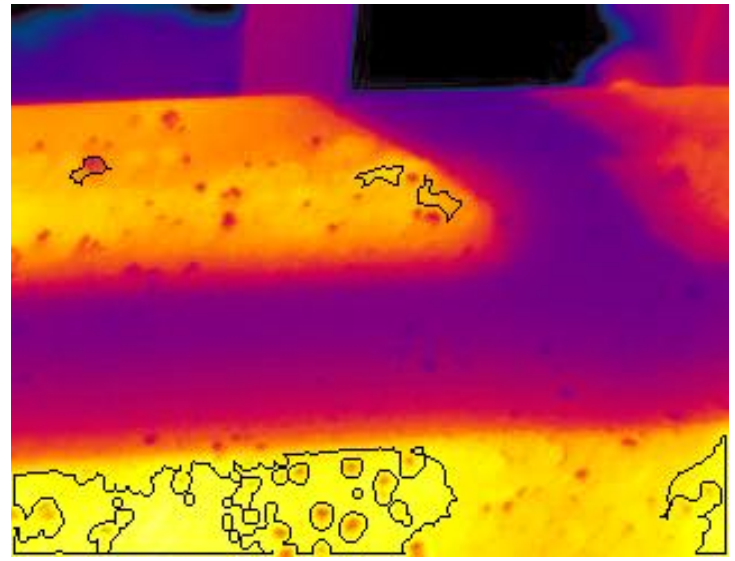

(b)

Figure 4.22: (a) Photograph of the guard rail area (b) Infrared image with the detected defects showing the shadow of the guard rail.

Irregularities such as dirt, oil spill, loose gravel result in an infrared image where the thermal distribution may resemble a defect area. Such images are shown in Figure 4.23(a)(b). The image in Figure 4.23(a) shows an infrared image created due to the presence of dirt and oil spill on the surface of the deck. Similarly the image in Figure 4.23(b) is created because of the presence of dirt and loose gravel. The white spots in the image resemble the thermal distribution of air filled defects. The algorithm however, is able to recognize that the white spots in Figure 4.23(b) are not defect areas.

\subsubsection{Test Conclusion}

The automated defect detection algorithm was able to locate debonds in the infrared images of the FRP deck accurately. However, when the infrared image had irregularities such as presence of dirt, gravel, obstruction due to guard rails etc, the algorithm falsely detected some regions in an infrared image as defect areas. These errors may decrease the overall accuracy of automated defect detection system. 


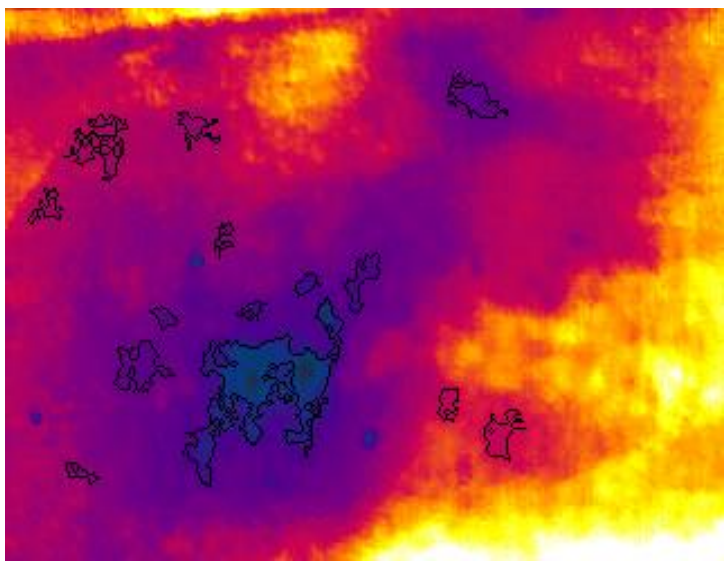

(a)

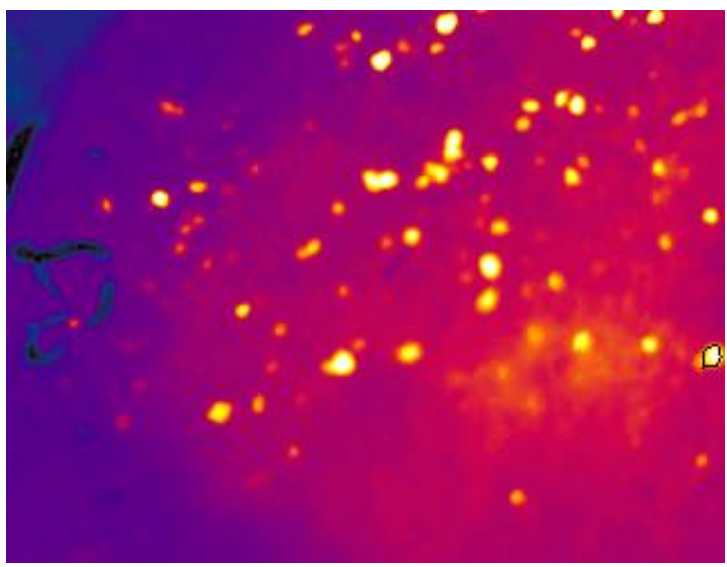

(b)

Figure 4.23: (a) Infrared image with dirt on surface (b) Infrared image showing dirt and loose gravel.

\subsection{Performance of Defect Detection Algorithms}

The results of automated defect detection of infrared images, tested under various conditions are shown in Figure 4.24(a) and (b). The conditions include different scenarios described in Section 4.5, with each scenario having 10 or more image sets. Some of the scenarios are: detecting debonds and delaminations filled with air, debonds and delamination with water, effect of changing distance etc.

Experiments using solar radiation as a passive heat source on the FRP bridge decks fabricated in the laboratory and on infrared images of a FRP bridge deck in operation have also been conducted successfully. The defect detection results are divided into three categories, as seen in Figure 4.24(a)(b). Defects that are recognized correctly in terms of position and size called "FULL" detected defects; defects that are located properly but their size is not correct are categorized as "PARTIAL" correct defects; defects that are present but are not termed as defects and defects that are not present but are termed as defects are categorized as "FALSE" detected defects. 


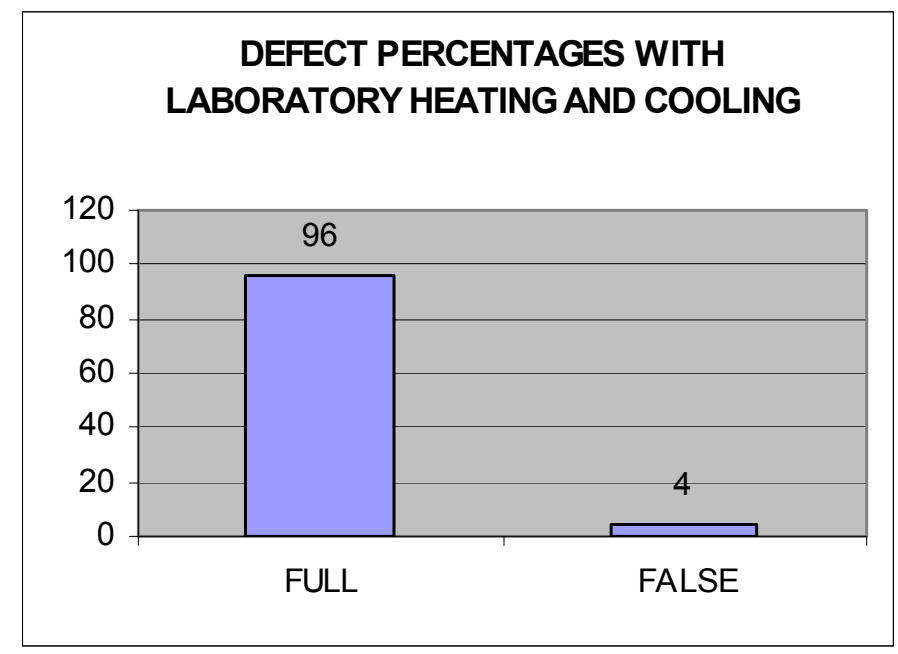

(a)

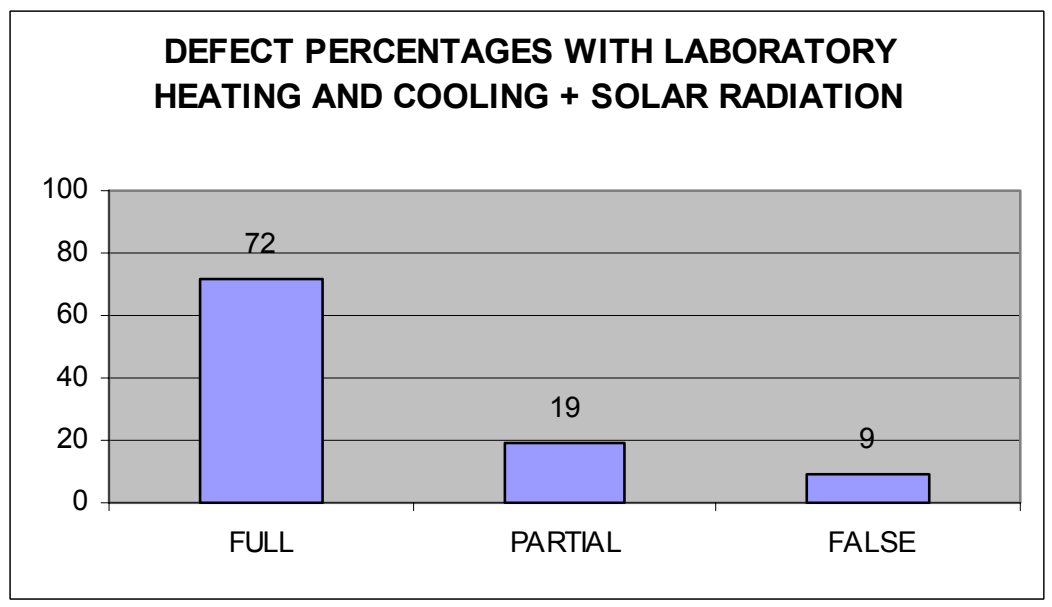

(b)

Figure 4.24: (a) Defect detection accuracy with laboratory heating and cooling. (b) Defect detection accuracy with laboratory heating and cooling and solar radiation.

Figure 4.24(a) shows the accuracy of defect detection percentages by experiments conducted under laboratory heating and cooling only. 96\% accuracy in detecting defects accurately (FULL) is seen in Figure 4.24(a). Figure 4.24(b) on the other hand shows accuracy of defect detection including results from experiments in the laboratory and under solar radiation. The percentage of FULL defect detection decreases to $72 \%$ with $19 \%$ of PARTIAL defect detection. Thus the effective accuracy of defect detection is 91\% with 9\% "FALSE" defect detection. This increase in FALSE defects may be due to 
reasons such as the position of the sun with respect to the deck causing uneven heating of the bridge deck. Hence solar radiation may cause an increase in errors in automated detection.

\subsection{Infrared Image Mosaicking Results}

Infrared image mosaicking was performed to generate a mosaic of the images that can be used to locate defects on the bridge deck as a whole. The requirements and advantages of mosaicking infrared images are discussed in Section 3.5. This section shows the results of the software and hardware assisted mosaicking algorithms.

\subsubsection{Software assisted Mosaicking}

The infrared images captured for defect detection cannot be used for mosaicking purposes since they cover the entire bridge deck area. It is also not possible to use infrared images from the field test on the FRP bridge since they were captured by a random camera movement. The assumption in the developed software for image mosaicking is that the camera is attached to a cart that moves in the horizontal and vertical direction. Hence the first task was to capture infrared images of FRP decks with movement only in the horizontal and vertical direction. These images will be used to mosaicking purposes. Figure 4.25 shows a sequence of infrared images from the same FRP deck. The three images cover the different areas of the bridge deck. Such a sequence of images is joined to generate an image mosaic.

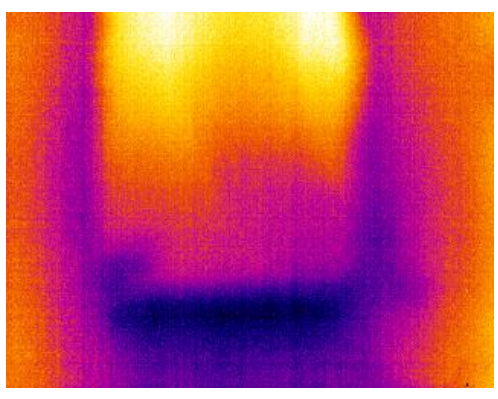

(a)

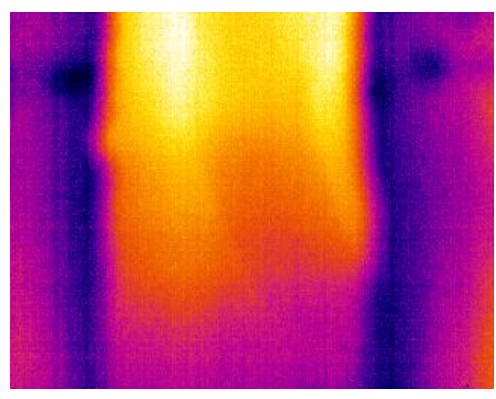

(b)

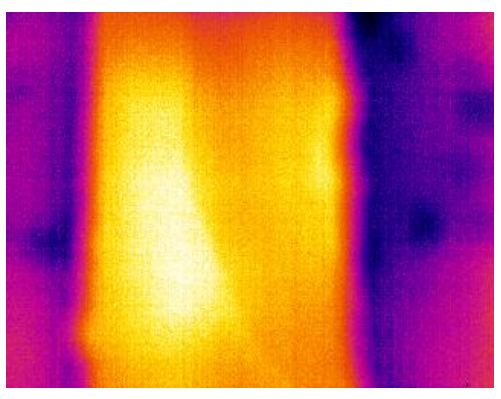

(c)

Figure 4.25 (a)(b)(c): Infrared images of the FRP bridge deck having movement only in the horizontal and vertical direction. 
Figure 4.26 shows the result of joining infrared images Figure 4.25(b) and Figure 4.25(c) to obtain an image mosaic. A perfectly mosaicked image is observed. On the other hand the image in Figure 4.27 shows an image mosaic obtained from images in Figure 4.25(c) and Figure 4.25(b). There is a slight registration error in the mosaicked image as seen from the figure. The error may be because of the ambiguous nature of edge information present in infrared images.

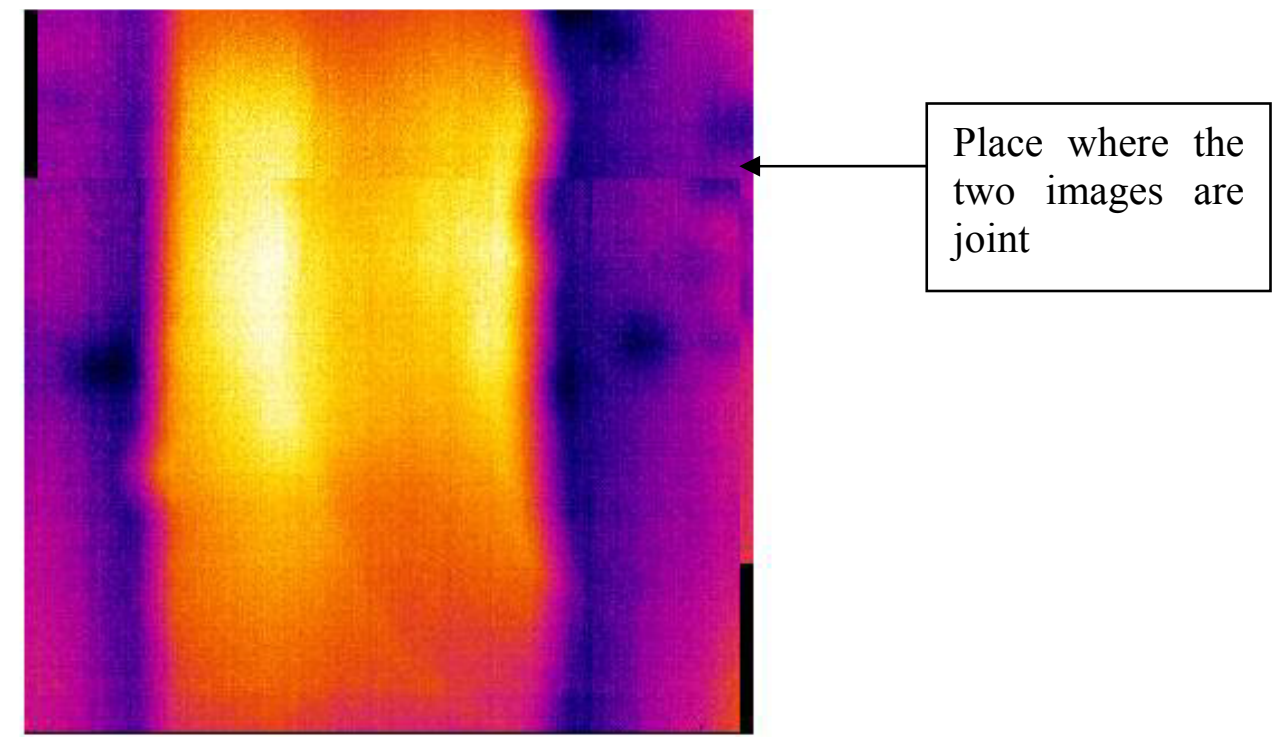

Figure 4.26: Result of mosaicking infrared images from Figure 4.25(b)(c)

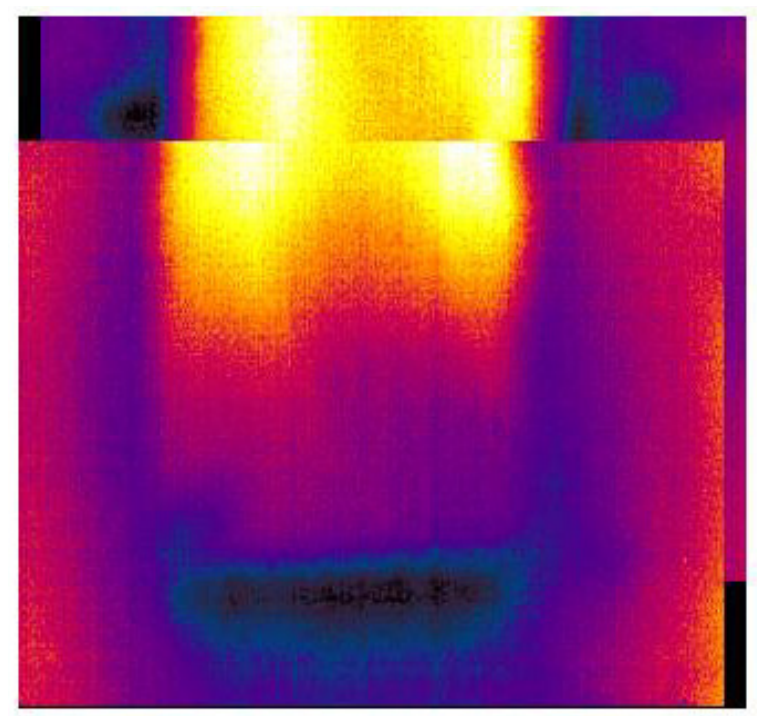

Figure 4.27: Result of mosaicking infrared images from Figure 4.25(a)(b) 
The mosaicking algorithm can be extended to mosaic a sequence of $\mathrm{N}$ images. Examples are shown in Figure 4.28(a) and (b). Figure 4.28(a) shows a mosaic obtained from the three images in Figure 4.25. The complete deck is not visible in this image. Hence more images are used to obtain a complete image mosaic as seen in Figure 4.28(b). This mosaic is obtained from a sequence of seven infrared images.

\subsubsection{Performance of Software assisted mosaicking}

Results from Figure 4.26, Figure 4.27 and Figure 4.28 show that infrared image mosaicking has been successfully performed. Image registration errors are found in some results such as the one in Figure 4.27. The reason for this error is the nature of the infrared images. The edge of the bridge decks is not clearly represented and has an overall faded look. This may introduce a registration error of a few pixels.

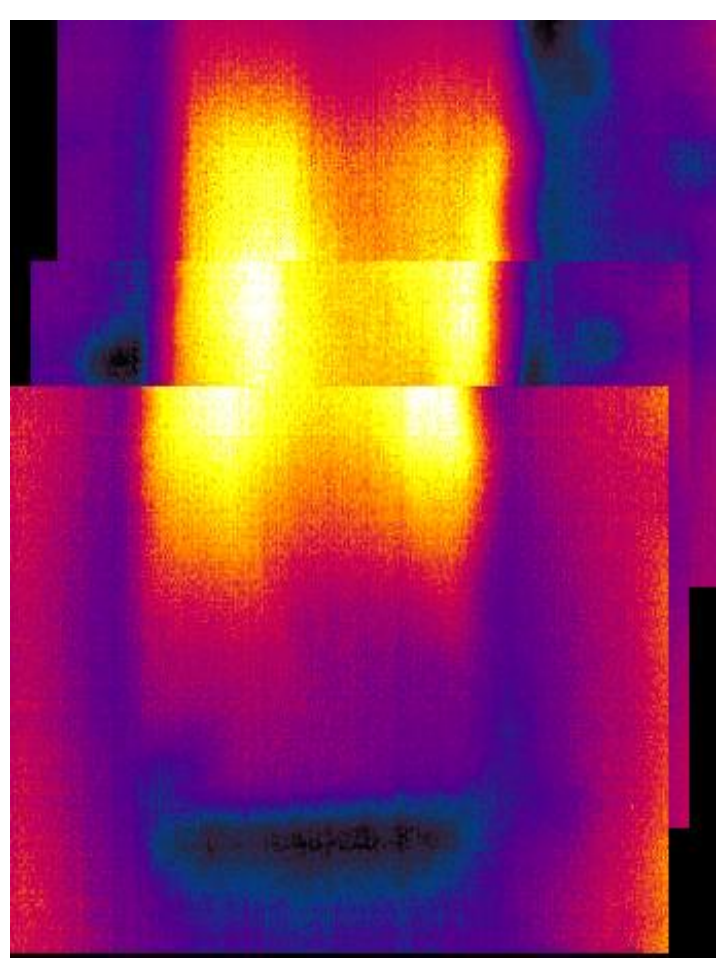

(a)

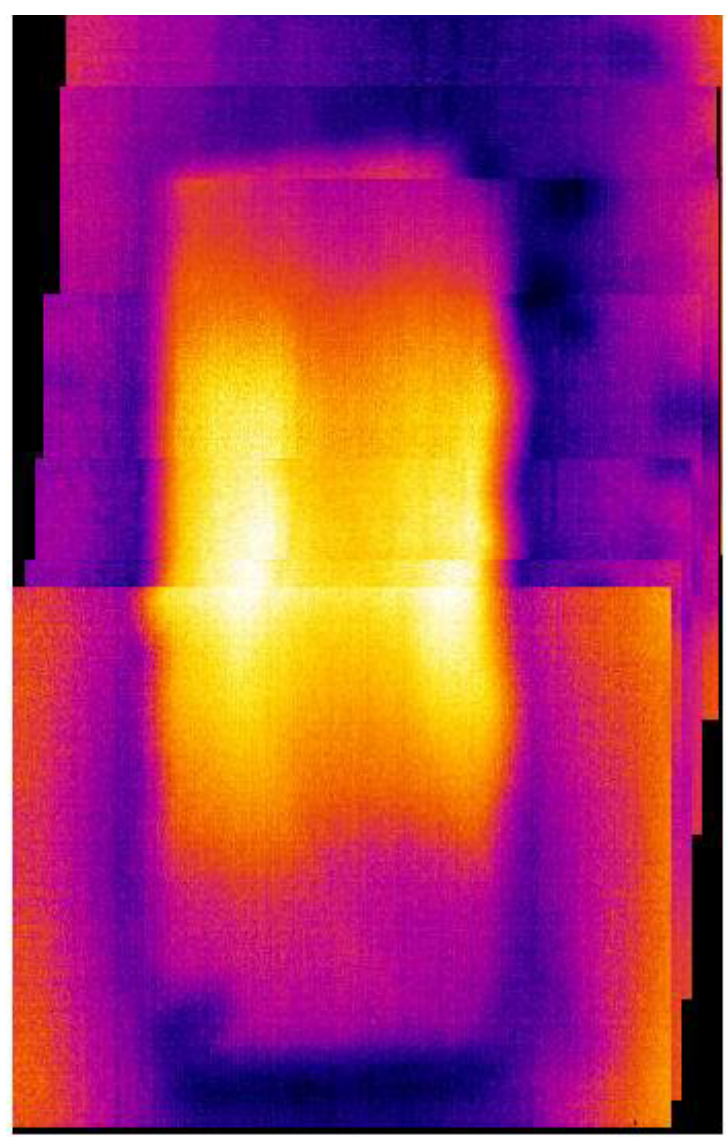

(b)

Figure 4.28: (a) Example of mosaicking 3 infrared images from a sequence. (b) Example of mosaicking 7 infrared images from a sequence. 
The time taken to mosaic two images is approximately 20 seconds which is too large for real time operation. Also, registration errors may decrease the accuracy of the mosaicking results. Hence the software assisted approach is deemed unacceptable for real time and accurate operation.

\subsubsection{Hardware assisted mosaicking}

In order to enable real time mosaicking of infrared images, hardware based mosaicking scheme was tested. The hardware assisted system takes feedback from sensors about the displacement (in $\mathrm{X}$ and $\mathrm{Y}$ direction) between two infrared images. The system is explained in detail in Section 3.5.1.

Infrared images of FRP bridge decks were captured in a sequence mentioned in 3.5.1. The camera angle was kept constant and images were captured at regular intervals. The images were joined together using the algorithm mentioned in Figure 3.12(a) to obtain the images shown in Figure 4.29 and Figure 4.31. The image in Figure 4.29 is a simulation of the hardware assisted mosaicking algorithm. The simulation was done by giving a set of input images to the hardware assisted algorithm and assuming the displacement parameters between the consecutive images. Defects were calculated in each infrared image using the automated defect detection algorithm. The images were then joined together to form a composite image.

The hardware assisted algorithms were also tested using a different approach. Six FRP decks each of approximately 2 feet $\mathrm{x} 1$ feet in size were aligned together as shown in Figure 4.30. The deck was heated using a quartz heater and infrared images of a part of the deck were captured in sequence. The white lines shown in Figure 4.30 are approximate boundaries of each infrared image from the entire joined deck. There are four images in each row and three rows in total. Thus the 12 infrared images when joined together will give a composite image of the entire FRP bridge deck. The final result of joining the 12 images using the hardware assisted mosaicking algorithm is shown in Figure 4.31. Since the images in this case were captured by holding the camera in hand, the boundaries were not well preserved. Because of this, the composite image in Figure 4.31 has some overlap between two consecutive images. This overlap will be eliminated 
if the process of acquiring infrared images can be automated using a cart with an infrared camera mounted on top. The cart will travel on a pre-programmed route and capture images at regular distance intervals.

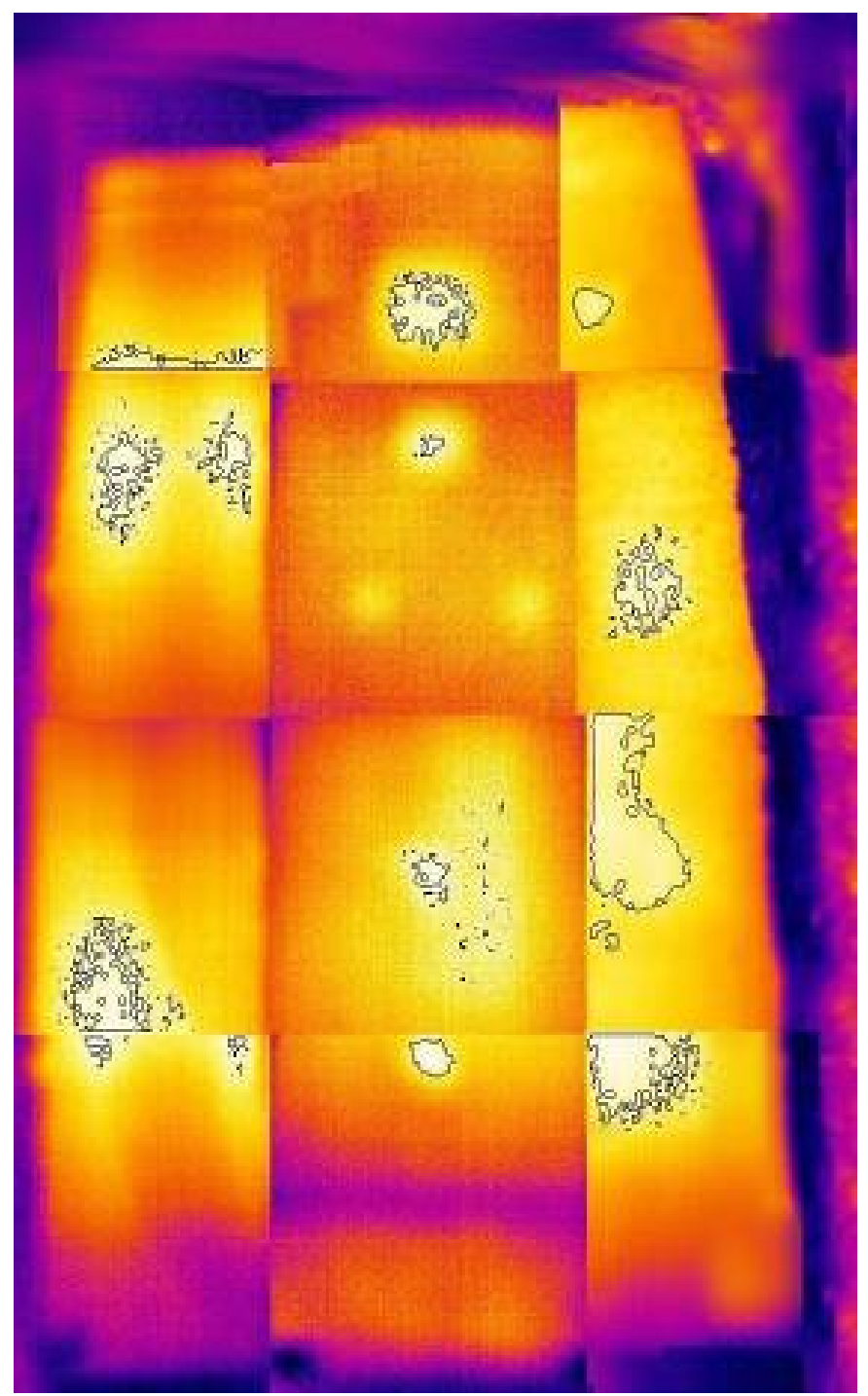

Figure 4.29: Result of hardware assisted mosaicking

\subsubsection{Performance of Hardware assisted Mosaicking}

The hardware assisted mosaicking uses feedback from sensors about the displacement between two consecutive infrared images. Hence, complex calculations to 
find the region of overlap are eliminated. Therefore real time operation becomes feasible. The current images have some registration errors mainly because in this case, the infrared camera was not mounted on a cart for automated operation. Hence there was some overlap between capturing images one after the other. When the process is entirely automated this overlap and registration errors may be negligible.

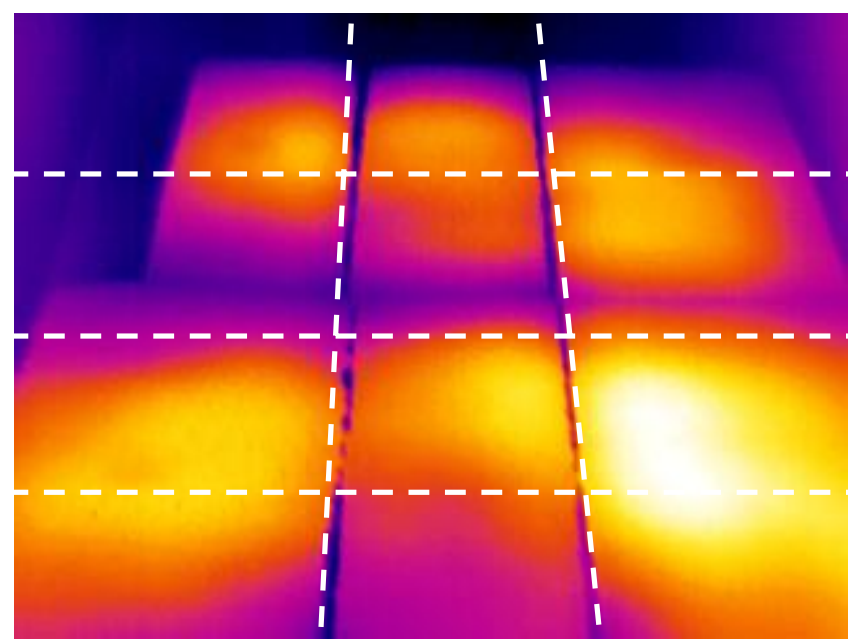

Figure 4.30: Setup for the hardware assisted mosaicking. Each polygon within the white dotted lines denotes the capture area of a single infrared image.

\subsection{Conclusions for Infrared Image Mosaicking}

Software and Hardware assisted approaches were tested to obtain an image mosaic. The advantage of the software assisted approach is that it can be extended to calculate a mosaic for any given set of input images irrespective of the camera angle and rotation between two consecutive images. However, the approach is computationally complex and hence not possible for real time implementation. Also, it has limitations in accurate mosaicking because of the nature of infrared images from GFRP decks. The images obtained are smooth with less information about corners or edges. This information is needed to detect control points robustly. Hence, even with small displacement between two images the control points detected could be different. This reduces the accuracy of the process of estimating translation and rotation parameters and a faulty mosaic map may be obtained. On the other hand the hardware assisted approach 
involves using sensors for calculating displacement parameters. The sensors will be attached to a cart that has an infrared camera mounted. A central controller will be used to monitor the feedback from the sensors and trigger image capture and mosaicking. Such a system can be easily implemented for a real time mosaicking of infrared images.

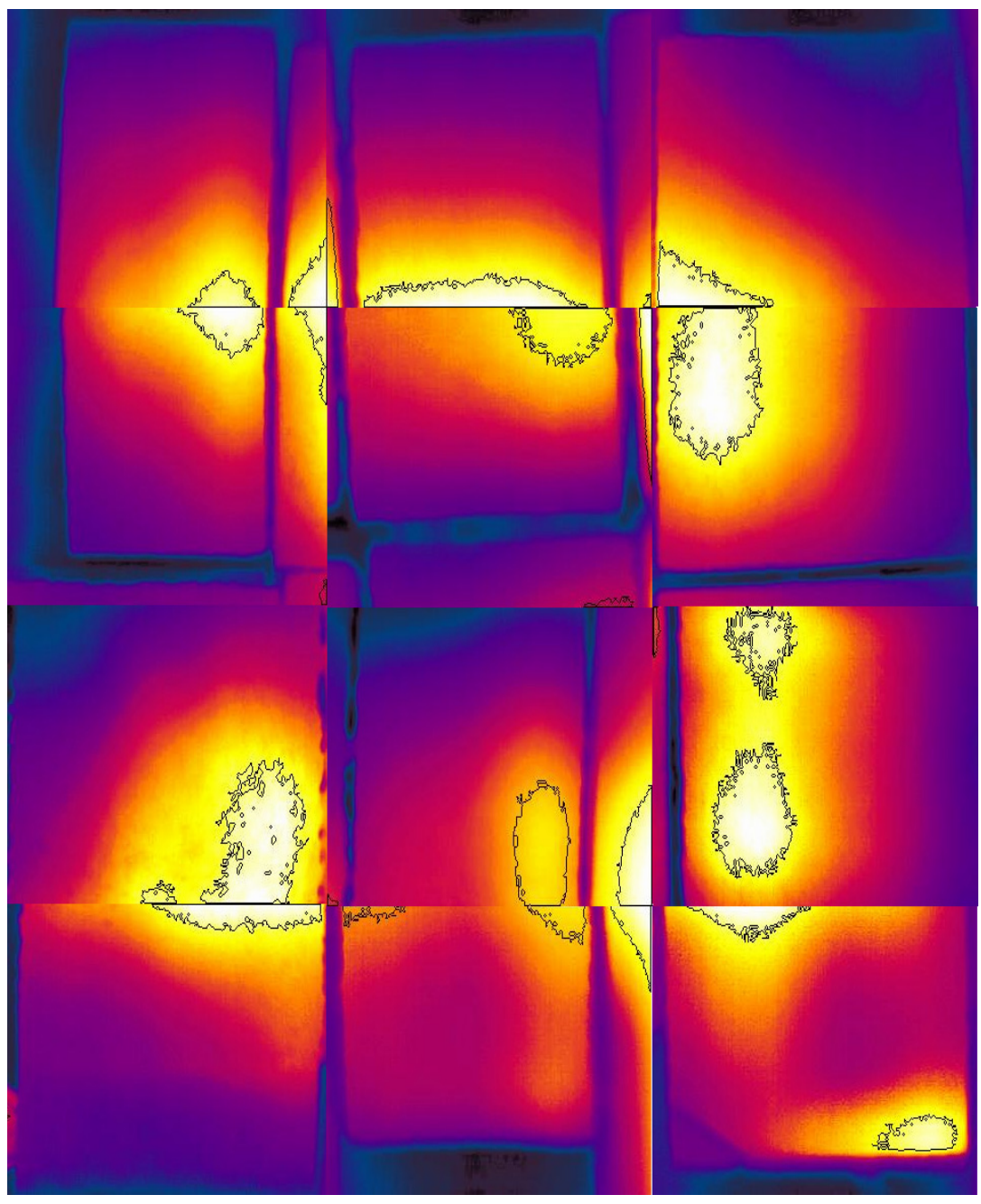

Figure 4.31: Result of hardware assisted mosaicking. 


\section{Chapter 5}

\section{Conclusions and Future Work}

\subsection{Conclusions}

The early detection of defects in a bridge is the main aim of any NDE system. A successful detection of defects in the early stages will help the maintenance personnel to monitor defect location and its potential growth so that pre-emptive action can be taken before any catastrophic failure. Also, early detection will help the monitoring agency such as state DOT's, save thousands of dollars needed to repair bridges having large size defects that may cause loss for structural integrity and lead to failure of the bridge.

The automated defect detection system developed in this work is a stepping stone for researchers interested in building a highly accurate defect detection system for periodic monitoring of FRP decks. The main challenge in designing such a system is not only to achieve or come close to the ideal detection accuracy of $100 \%$, but also to minimize false defect detection. A detection of false defects causes monetary losses to the government agencies that maintain civil and military infrastructure.

Successful implementation of the prototype turn-key system that is able to interface with the infrared camera, capture and analyze images was achieved as a part of this research. It was shown that automated detection of defects can lead to a system where it is easier to visualize the results and take pre-emptive action. The results show that the system has performed with an accuracy of $90 \%$ after testing under variations conditions such as variation in the size of defects, variation in distance, variation in 


\section{CHAPTER 5. CONCLUSIONS AND FUTURE WORK}

heating/cooling conditions etc. However, there is some limitation on the detection of defects that are 1"xl" in size or less. The small size defects are not able to create a distinguishable temperature gradient (approximately greater than $2^{\circ} \mathrm{C}$ ) needed for defect detection.

Experiments have shown that care must also be taken to operate such an automated defect detection system under solar radiation. This reason for this is that solar radiation can lead to uneven heating of the decks that result in a thermal patter different from that generated by actual defects. This increases the chances of FLASE defect detection, thus decreasing the accuracy of the algorithms. It was estimated that the best times to use such a system under solar radiation are between $12: 00 \mathrm{pm}$ to $3: 00 \mathrm{pm}$.

FRP bridges under operation will have materials such as dirt, gravel and oil on their surface. It is necessary to minimize their effect on the automated system by cleaning the bridges before capturing images by infrared thermography. These obstructions could affect the thermal patterns and can lead to detection of a FALSE defect.

The second part of the research explored various methods for mosaicking infrared images of GFRP bridge decks to build a "composite image" mosaic. The use of "composite image mosaic" will help the technicians to point out the exact location of defects on the bridge deck. Mosaicking of infrared images is a challenging task because of their nature. The lack of edge information in these images may impose some restriction on infrared image mosaicking.

Hardware and software assisted approaches were explored in this work for mosaicking infrared images. A software assisted system was developed to mosaic a given set of infrared images to form a single image and defect map of the bridge deck. The disadvantage of the software assisted system is the computational complexity. It limits real time operation of the mosaicking scheme. Alternatively, the hardware assisted mosaicking method is found to be fast and is computationally efficient. It will enable real time mosaicking and defect detection of infrared images. 


\section{CHAPTER 5. CONCLUSIONS AND FUTURE WORK}

\subsection{Future Work}

Until the ideal $100 \%$ or near $100 \%$ accuracy is achieved, algorithms and methods for automated defect detection need refining. The current system has not been able to achieve the $100 \%$ goal. Hence future work is necessary to refine the algorithms or develop new ones. The main requirement for validating any defect detection algorithm is that of infrared images. It was found that laboratory testing yielded high defect detection accuracy. This accuracy decreased when results from infrared images of bridges exposed to solar radiation were taken into consideration. However, the amount of data required for validation of algorithms under the presence of solar radiation was limited. Image data can play an important role in validating the accuracy of the developed algorithms. Hence, it may be necessary to periodically capture infrared images from an in service FRP bridge decks to build a database of infrared images and train the computer to detect true defects from false ones. The images in the database can be classified according to the defect type (debonds or delaminations), environmental conditions (temperature, exposure to solar radiation etc.) among other methods. Such a categorized database when used for laboratory testing of any algorithm developed for automated detection, will yield results similar to those obtained in field conditions under the presence of dust, gravel, oil spills, uneven heating etc. That is, the accuracy of the algorithm tested under such a database can be a true indicator of the accuracy obtained in field conditions. This is a probabilistic approach to building a robust automated defect detection system. Another method is that of using a deterministic approach where the thermal response of FRP bridge decks under varying conditions can be modelled using laws of physics. Such an approach can lead to better understanding of the development and growth of defects in FRP bridge decks. In the long run, the probabilistic and deterministic approaches can be combined to build a robust automated defect detection system that is able to locate defects and monitor as well as estimate their growth and severity.

The current system is able to detect defect location, but not their depth from the surface. Some research to calculate the defect depth using the development of the thermal profile over a time period has been carried out by [2]. This possibility can be explored to pin point the exact depth of defects. The use of neural networks for automated defect 


\section{CHAPTER 5. CONCLUSIONS AND FUTURE WORK}

detection can be explored; for example as accomplished by Kohenen SOM (Self Organizing Maps). Research to design a fuzzy logic system that can give a characteristic evaluation such as severity of the defect, effect on bridge deck etc can be carried out. More research needs to be performed on the errors that can occur by using an automated system on FRP bridge decks in operation. Compensation for these errors if any is needed to make the automated defect detection system more robust and reliable for field applications.

The current defect detection system uses far infrared (6-15 $\mu \mathrm{m})$ to capture infrared images. There could be limitations in using infrared thermograpy or using only the far infrared band for automated analysis of defects. Multimodal approaches using more than one imaging source or image features can lead to a more robust automated defect detection system. Such a multimodal approach can be carried out in two ways. One method is to capture infrared images in the near infrared $(0.75-3 \mu \mathrm{m})$ band as well as the far infrared $(6-15 \mu \mathrm{m})$ band. The images in the near infrared band have features in between those of visible images and images from the far infrared band. These features (from the near infrared image) can be used to reduce errors and increase detection accuracy. The other method is to combine the results of infrared thermography with that of another NDE technique such as GPR. Such a system has been explored by Maser K. [55]. Future work also includes developing the sensor hardware for completing the hardware assisted mosaicking setup to enable real time mosaicking. This system can be used in conjunction with a ground penetrating radar based system for defect detection. A robot can be built and programmed to automatically acquire infrared images and perform automated analysis as well as image mosaicking. 


\section{References}

1. National Bridge Inventory 2003 - http://www.fhwa.dot.gov/bridge/nbi.htm

2. Ansari F. (Editor), "Condition Monitoring of Materials and Structures," ISBN 078440495X, ASCE, 2000.

3. Tang B, "Fiber Reinforced Polymer Composites Applications in USA," First Korea/U.S.A. Road Workshop Proceedings, January 28-29, 1997

4. Roy M, "Nondestructive Testing Of FRP Structural Components Using Digital Infrared Thermography," M.S. Problem Report, Department of Civil and Environmental Engineering, West Virginia University, Morgantown, WV 26505, July 2004.

5. Holbert R, "The importance of Bridge inspections," Sunday Gazette Mail, February 15th 1998.

6. Halabe U. B., Martinelli D. R., Petro S., "Enhancing quality control in transportation construction through non-destructive testing," Nondestructive Evaluation of Aging Materials and Composites III, Newport Beach California, March 1999.

7. Halabe U. B., "Nondestructive Material and Structural Evaluations," CE 364 Course Package, Department of Civil and Environmental Engineering, West Virginia University, Morgantown, WV, 161-176, 1999.

8. Maldague X., "Nondestructive testing Handbook Volume 3, "Infrared and Thermal Testing," publisher ASNT, ASNT, 2000.

9. Maldague P.V. "Nondestructive Evaluation of Materials by Infrared Thermography" Springer-Verlag 1993, ISBN 3-540-19769-9.

10. Vasudevan A, "Application of Digital Infrared Thermography for Nondestructive Evaluation of composite bridge components," M.S. Thesis submitted to Department of Civil and Environmental Engineering, West Virginia University, Morgantown, WV - 26505, January 2004.

11. FLIR ThermaCAM S60 Datasheet, "Operators Manual SDK 2.5," http://www.flir.ca/support/infrared_software_updates.asp\#id27

12. Banglore G. S., "Nondestructive Evaluation of FRP composite members using Infrared Thermography," M.S. Thesis submitted to Department of Civil and Environmental Engineering, West Virginia University, WV 26505, May 2002.

13. Kaplan, H., "Practical Applications of Infrared Thermal Sensing and Imaging Equipment," Second Edition, SPIE Optical Engineering Press, Bellingham, WA 1999.

14. Maldague X., "Applications of Infrared Thermography in Nondestructive Evaluation," http://www.gel.ulaval.ca/ maldagx/r_1123.pdf, 2003.

15. Maldague X., "Introduction to NDT by Active Infrared Thermography," http://www.gel.ulaval.ca/ maldagx/r_1221t.pdf, 2003.

16. Vanzetti R., "Practical Applications of Infrared Techniques," ISBN 0471903604, John Wiley \& Sons Inc, December 1972. 
17. Halabe U. B., Maser K. R., "Thermal Modeling of a Leaky Roof," MIT CCRE Technical Note, March 1986.

18. Alqennah H., "Detection of subsurface anomalies in composite bridge decks using infrared thermography." MS Thesis, Department of Civil and Environmental Engineering, 2000, West Virginia University, Morgantown, WV.

19. Miceli M., "Assessment of Infrared Thermography as NDE Method for Investigation of FRP Bridge Decks." M.S. Thesis, Department of material Science and Engineering, Virginia Polytechnic Institute and State University, November 2000.

20. Miceli, M., Horne, M. R., and Duke, J. C. (2001). "Health monitoring of FRP bridge decks." Advanced Nondestructive Evaluation for Structural and Biological Health Monitoring, Proc. SPIE - Volume 4335, Tribikram Kundu ed., March, p. 100-105.

21. Halabe U. B., Vasudevan A., GangaRao H. V. S., P. Klinkhachorn, "Subsurface Defect Detection in FRP Composites Using Infrared Thermography," Quantitative Nondestructive Evaluation 2004, Golden, Colorado.

22. Halabe U. B., Roy M., GangaRao H. V. S., "Detection of Air and Water-Filled Subsurface Defects in FRP Bridge Decks Using Infrared Thermography," Quantitative Nondestructive Evaluation 2005, Brunswick, Maine.

23. Maldague X, Krapez J. C., Poussart D., "Thermographic Nondestructive Evaluation (NDE): An Algorithm for Automatic Defect Extraction in Infrared Images," IEEE Transactions on Systems Man and Cybernatics, Volume 20, June 1990.

24. Plotnikov Y. A., Winfree W. P., "Visualization of subsurface defects in composites using a focal plane array infrared camera," Thermosense XXI, Orlando, FL, 1999, SPIE Proceedings Vol. 3700.

25. Lhota J. R., Shepard S. M., Bruce A., Ahmed, T., "Enhanced spatial and depth resolution of pulsed thermographic images," Review of Progress in Quantitative Nondestructive Evaluation, Volume 20A, AIP Conference Proceedings 557, Amens, Iowa, 2000, p. 492-498.

26. Shepard S. M., Rubadeux B. A., Ahmed, T., "Automated thermographic defect recognition and measurement," Nondestructive Characterization of Materials IX, AIP Conference Proceedings 497, Sydney, Australia, 1999, p. 373-378.

27. Shepard S. M., Lhota J. R., Rubadeux B. A., Ahmed T., Wang D., "Enhancement and reconstruction of thermographic NDT data," Thermosense XXII, Vol. 4020, March 2000.

28. Rigney M. P., Franke E. A., "Machine Vision applications of low-cost thermal infrared camera," Thermosense XXIV, Volume 4710, April 2002.

29. Allred .G., Jones M. H., "The need for image processing in infrared camera design," Thermosense XXIV, Volume 4710, April 2002.

30. Heil . B. W., "More Than Defect Detection - The Real Value of Web Inspection," ISRA surface vision, www.isravision.com.

31. Miittal A., Biswas S., "Pultrusion of Composites - An Overview," http://www.tifac.org.in/news/pultr.htm

32. FLIR ThermaCAM S60 http://www.flirthermography.com/cameras/camera/1063/ 
33. FLIR ThermaCAM Researcher software datasheet. http://www.flirthermography.com/media/software/Researcher\%20Datasheet.pdf

34. "A Turnkey Test System for IR Imaging," Photonics Tech Briefs, May 1999.

35. Allred L. G., Jones M. H., "The need of image processing in Infrared Camera Design," Thermosense XXII Orlando Florida, 2000.

36. Brown L. G., "A survey of image registration techniques," ACM Computing Surveys, 24(4):325-376, 1992.

37. Hines G. D., Rahman Z., Jobson D. J., Woodell G. A., "Multi-image registration for an enhanced vision system," Visual Information Processing XII, Proceedings of the SPIE, Volume 5108, pp. 231-241 (2003).

38. Li H., Zhou Y. T., Chellappa R., "SAR/IR Sensor Image Fusion and Real-Time Implementation," Signals, Systems and Computers, Volume 2, pg. 1121 - 1125 1995.

39. Eustice R., Pizarro O., Roman C., Singh H., "Underwater image processing toolbox for optical image processing and mosaicking in Matlab,", Proceedings of the 2002 International Symposium on Underwater Technology, pg. 141 - 145, April 2002.

40. Burt and Adelson, "A Multiresolution spline with application to image mosaics," in ACM Trans. on Graphics, pp.2(4):217--236, October, 1983.

41. Koschan A., Ng J. C., Abidi M., "Multi-perspective Mosaics for Under Vehicle Inspection," Proceedings of SPIE Unmanned Ground Vehicle Technology VI, Vol. 5422, Orlando, FL, USA, pp. 1-10, April 2004.

42. Giardina C. R., Dougherty E. R., "Morphological Methods in Image and Signal Processing”, Prentice Hall, 1988, ISBN o-13-601295-7.

43. Meyer, F. and S. Beucher, Morphological Segmentation. J. Visual Comm. Image Rep., 1990. 1(1): p. 21-46.

44. Young I.T., Gerbrands J.J., Vliet L.J., "Fundamentals of Image Processing," online tutorial at http://www.ph.tn.tudelft.nl/Courses/FIP/noframes/fip.html

45. Gillespie A. R., Kahle A. B., Walker R. E., "Color Enhancement of Highly Correlated Images: Decorrelation and HSI Contrast Stretches," Remote Sensing of Environment, Volume 20, pp. 209-235, 1987.

46. TNT products Reference Manual : http://wwwzoo.im.gda.pl/mips/proci003.htm

47. Alley R. E., "Algorithm Theoretical Basis Document for Decorrelation Stretch," Jet Propulsion Laboratory, 4800 Oak Grove Drive, Pasadena, CA 91109, August 1996.

48. Horváth, J., "Image Segmentation Using Clustering," Proceedings of 3rd Doctoral Conference, pg 105-106, 2003, ISBN 80-968666-3-X

49. Jiang L., Yang W., "A modified Fuzzy C-Mean Algorithm for Segmentation of Magnetic Resonance Images," Digital Image Computing - Techniques and Applications (DICTA), Sydney, Australia, December 2003, pg225-232.

50. Gumustekin S., “An Introduction to Image Mosaicking,” July, 1999.

51. Klinkhachorn P., Lonkar G. M., Halabe U. B., Hota V. S. G., "Real time detection of Defects In GFRP bridge decks using Infrared Thermography," Quantitative Nondestructive Evaluation 2005, Maine, New York.

52. Harris C. and Stephens M., "A Combined Corner and Edge Detector," Proceedings of Fourth Alvey Vision Conference, Vol. 15, 1988, pp. 147-151. 
53. AutoIT software. http://www.hiddensoft.com/AutoIt

54. FLIR ThermoVision SDK : http://209.162.248.118/index_files/Page466.htm

55. Maser K.R., Horschel, M. G., "Integration of GPR and Infrared Thermography for Bridge Deck Assessment," Proceedings of ASNT Conference on NDE/NDT for Highways and Bridges, Cincinnati, OH, September 2002. 


\section{Appendix A}

This appendix explains the functionality and usage of the GUI developed for automated detection of defects.

\section{GUI for Automated Defect detection}

The GUI for Automated defect detection is shown in Figure A.1 along with the explanation for each component.

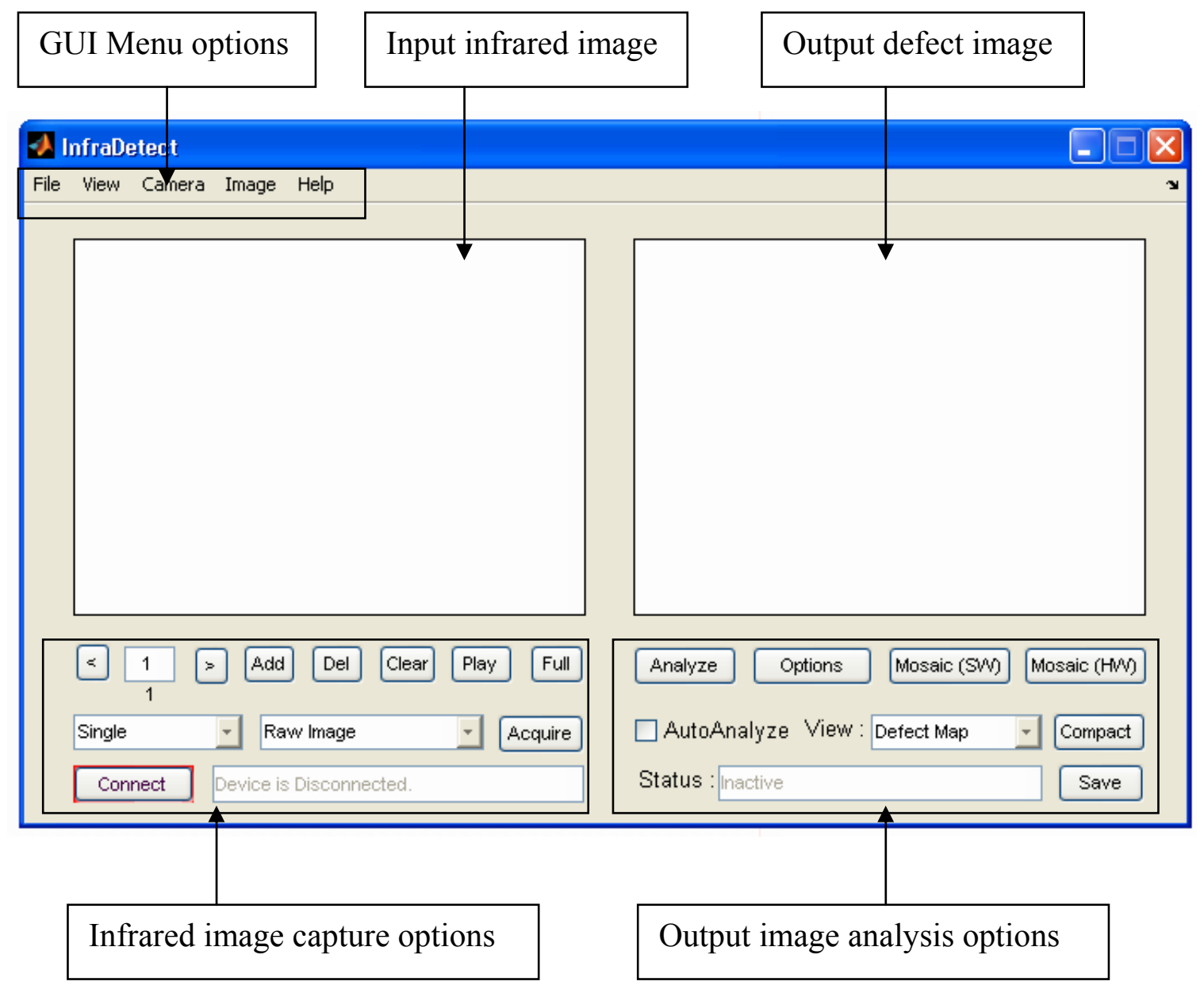

Figure A.1: GUI for automated defect detection 
The basic steps to run the GUI are as follows:

1. Click Connect button.

2. Wait till Status shows "Device is Connected".

3. Click File Tab and select the type of file to be read. The type of files that can be read are:

a) Infrared image sequence file (.seq file).

b) Single Infrared Image (.jpeg file).

4. Select the File to be read. The infrared image will be displayed in the GUI.

5. Select the Analysis Options.

6. Click "Analyze" to analyze for defects.

A sample run of the GUI after executing the above steps is shown in Figure A.2. The binary defect map of the detected defects in the infrared image is shown in the output axis.

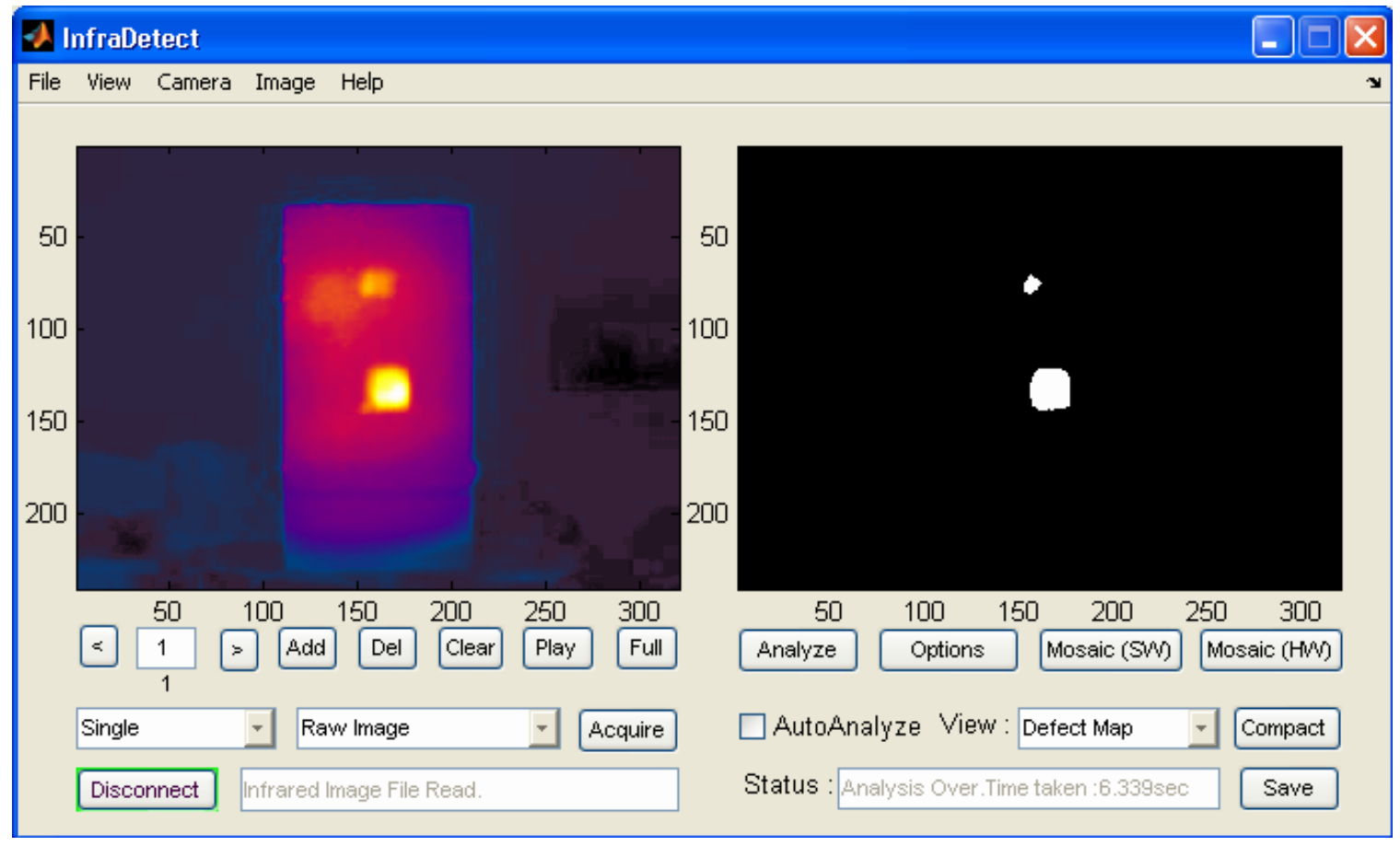

Figure A.2: Sample run of the GUI 


\section{Explanation of GUI Options}

The functioning of the GUI options along with their explanation is given in Figure A.3 and Figure A.4.

Add: Adding image for defect detection

Del: Deleting image from defect detection queue.

Clear: Clearing input image axis.

Play: Playing the set of image selected for defect detection.

Full: Full view of the GUI.

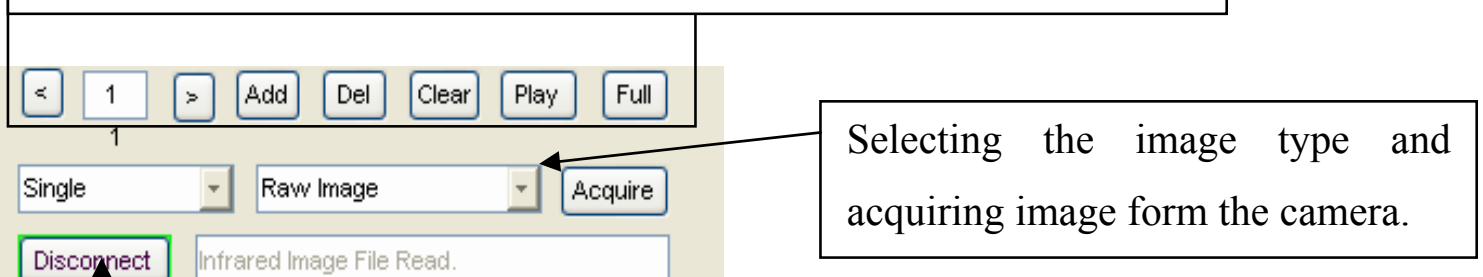

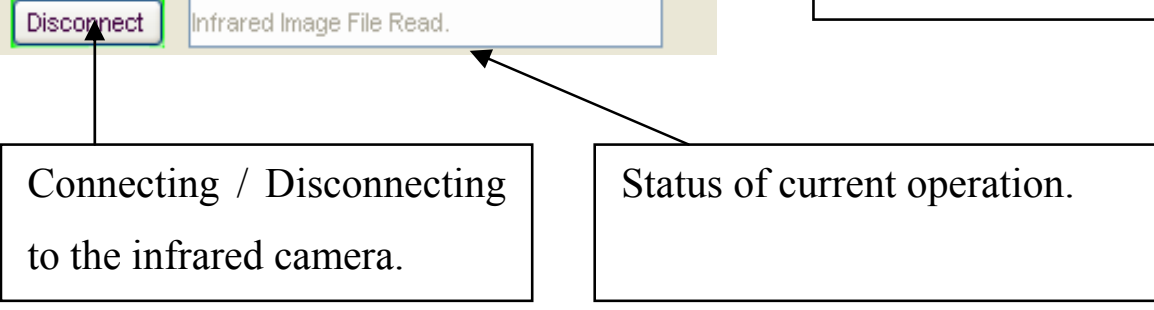

Figure A.3: Input Options in the GUI

Analyze: Analyzing the current image for defects.

Options: Selecting the defect detection options.

Mosaic (SW): Initializing software based mosaicking GUI.

Mosaic (HW): Initializing hardware based mosaicking GUI.

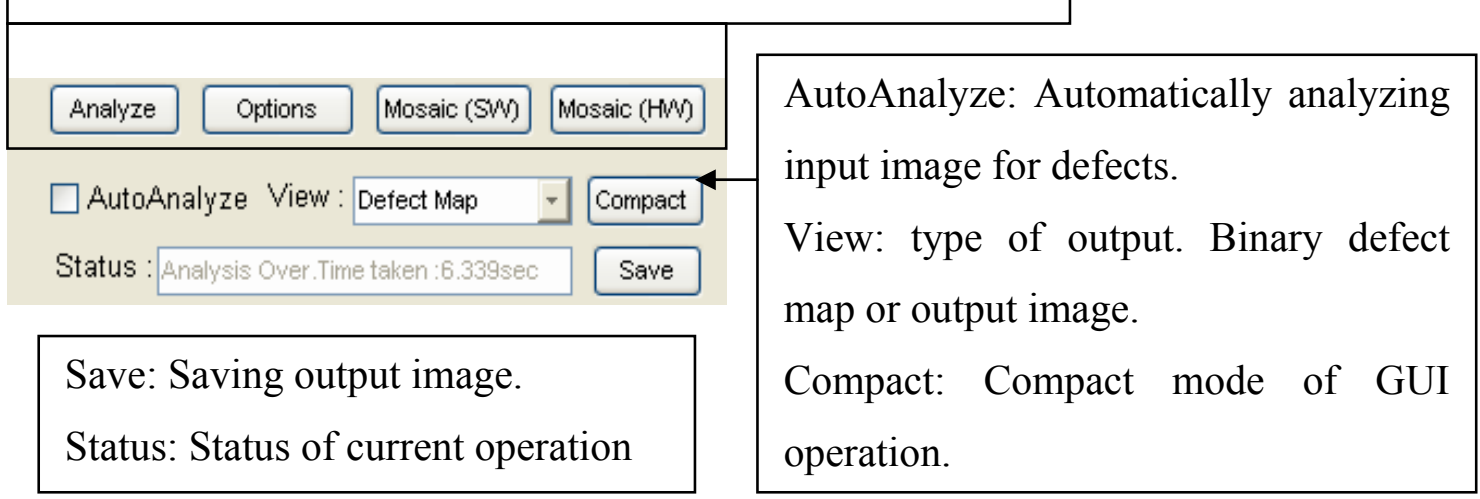

Figure A.4: Output Options in the GUI. 


\section{Analysis Options}

There are options that can be selected for automated analysis of defects in infrared images. For example, three algorithms have been developed for the automated detection of defects. They are explained in detail in Figure A.5.

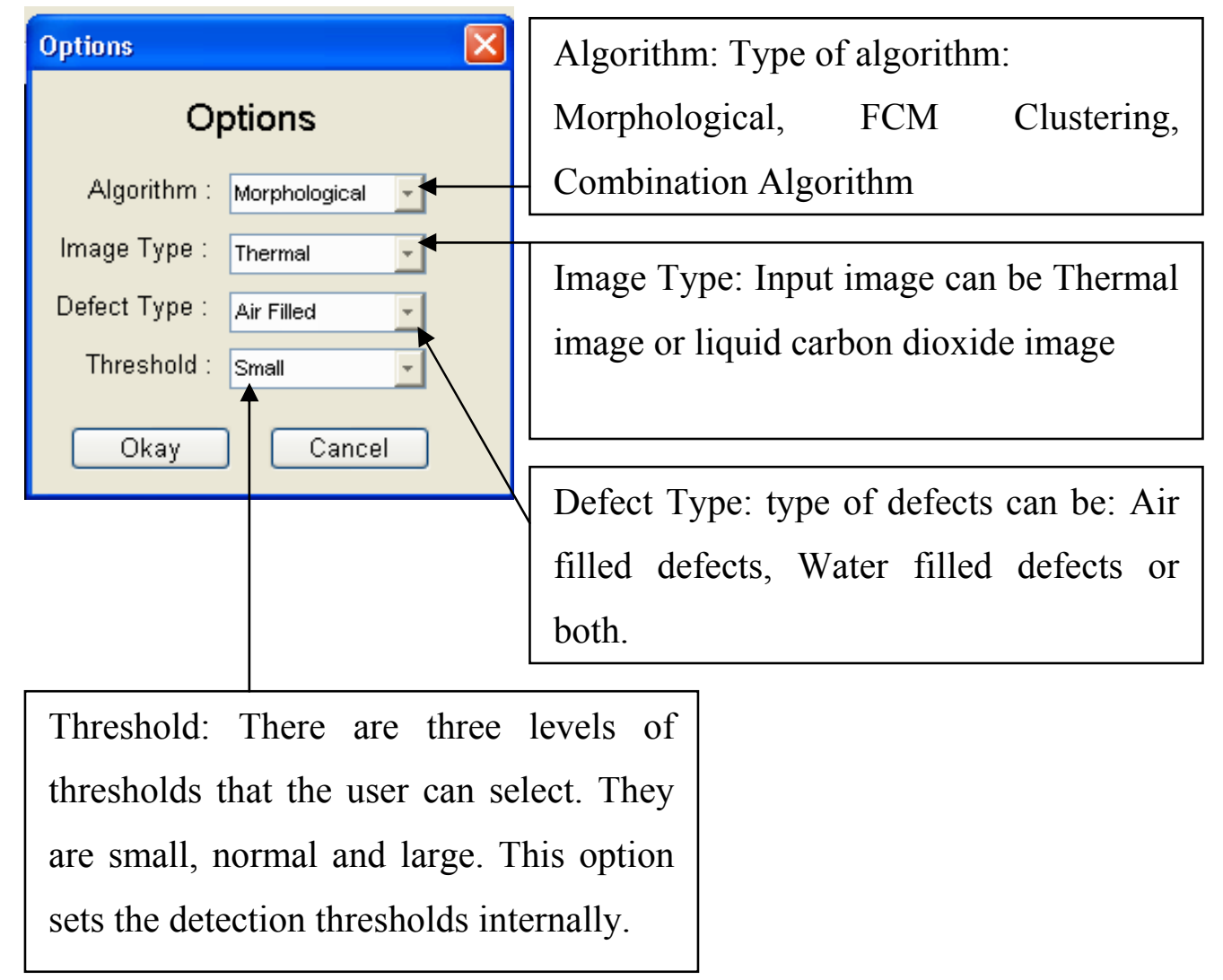

Figure A.5: Analysis options.

\section{Menu Options}

The various menu tabs are used to perform different tasks such as connecting to the infrared camera, adjusting the camera etc. Their explanation is given in Figure A.6 to Figure A.9. 


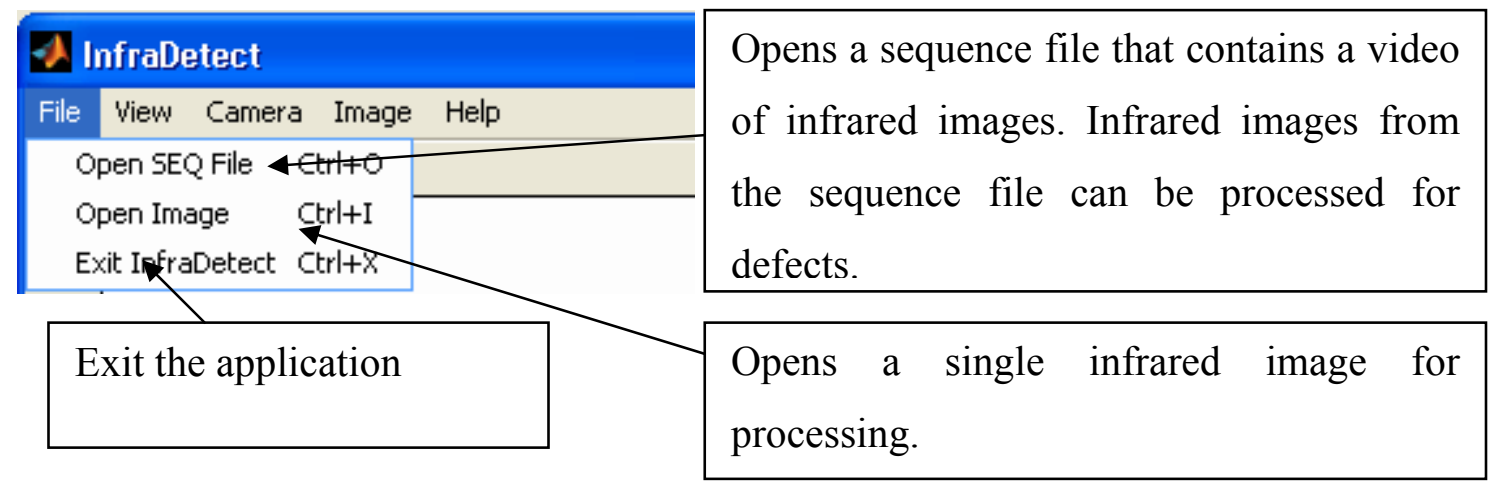

Figure A.6: File Menu options.

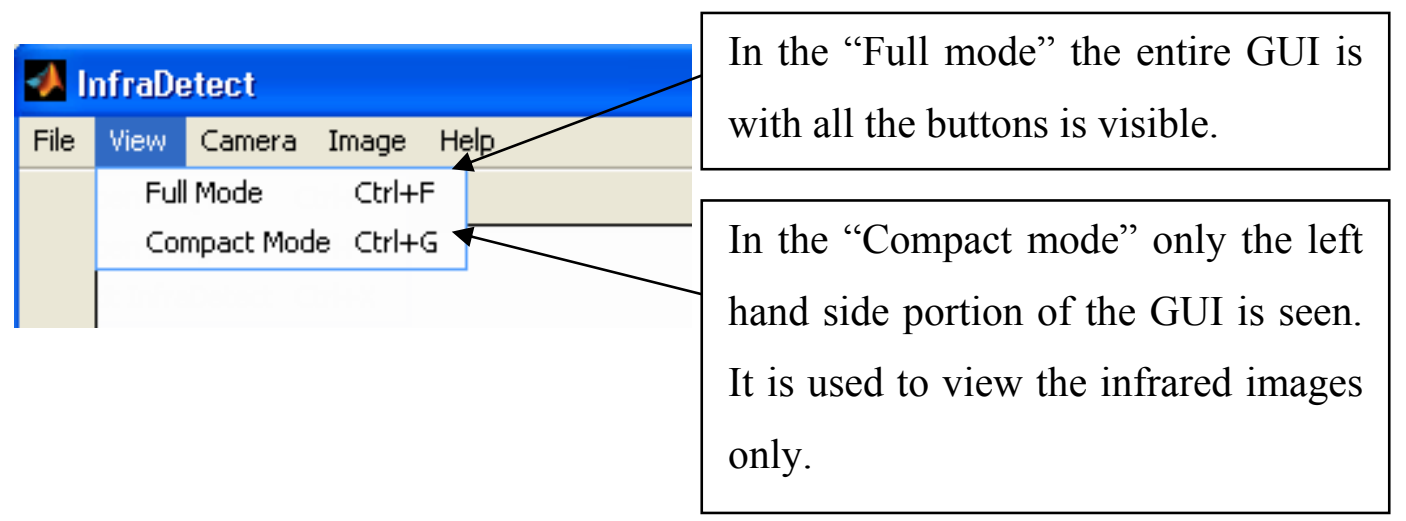

Figure A.7 View menu options

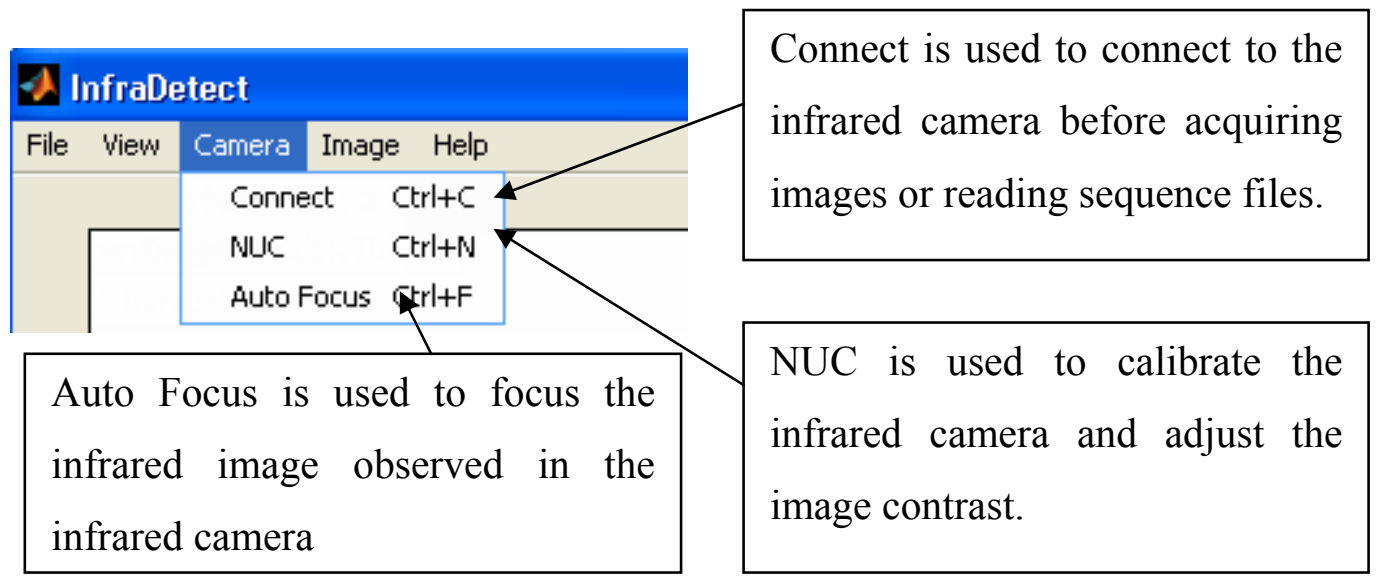

Figure A.8: Camera menu options. 


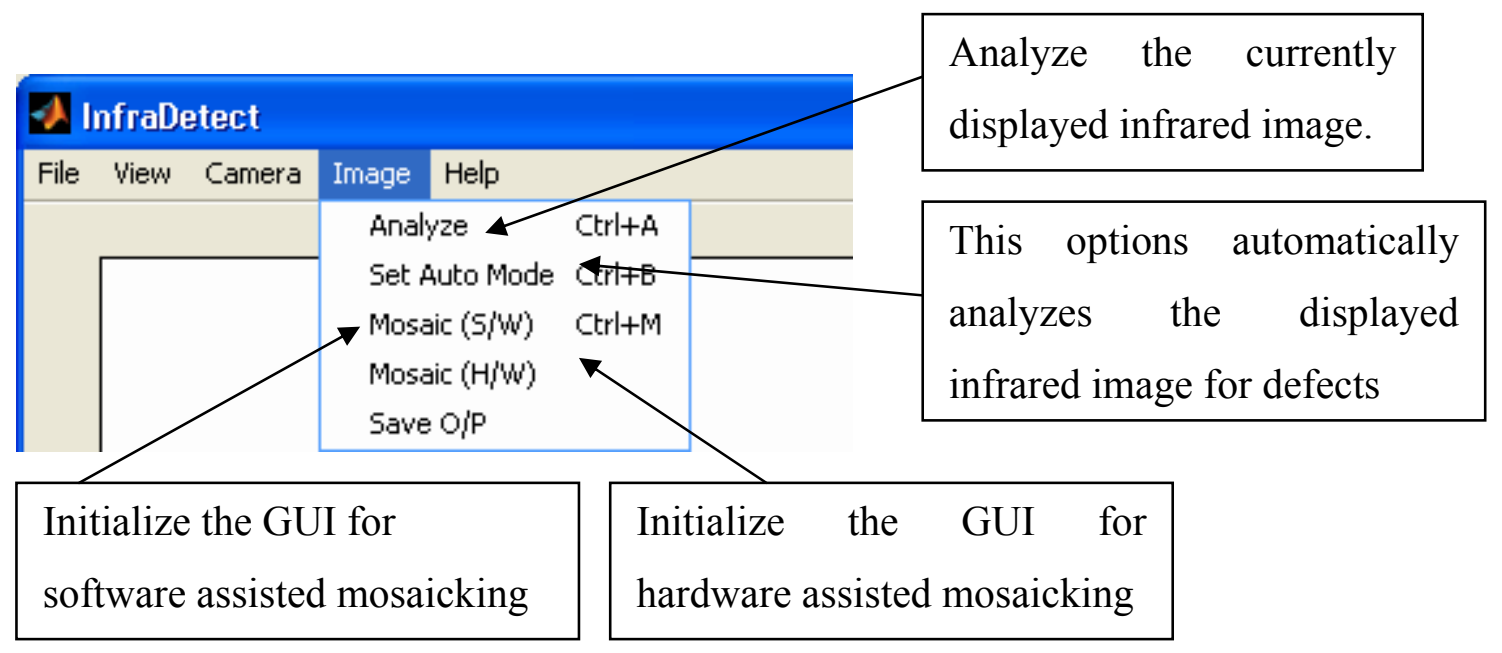

Figure A.9: Image menu options.

\section{Mosaicking GUI}

The GUI for hardware/software assisted mosaicking is shown in Figure A.10. It is seen to be similar to the defect detection GUI shown in Figure A.1. The main difference is that the Mosaic button is used to obtain a composite image mosaic of the set of images stored selected by the user.

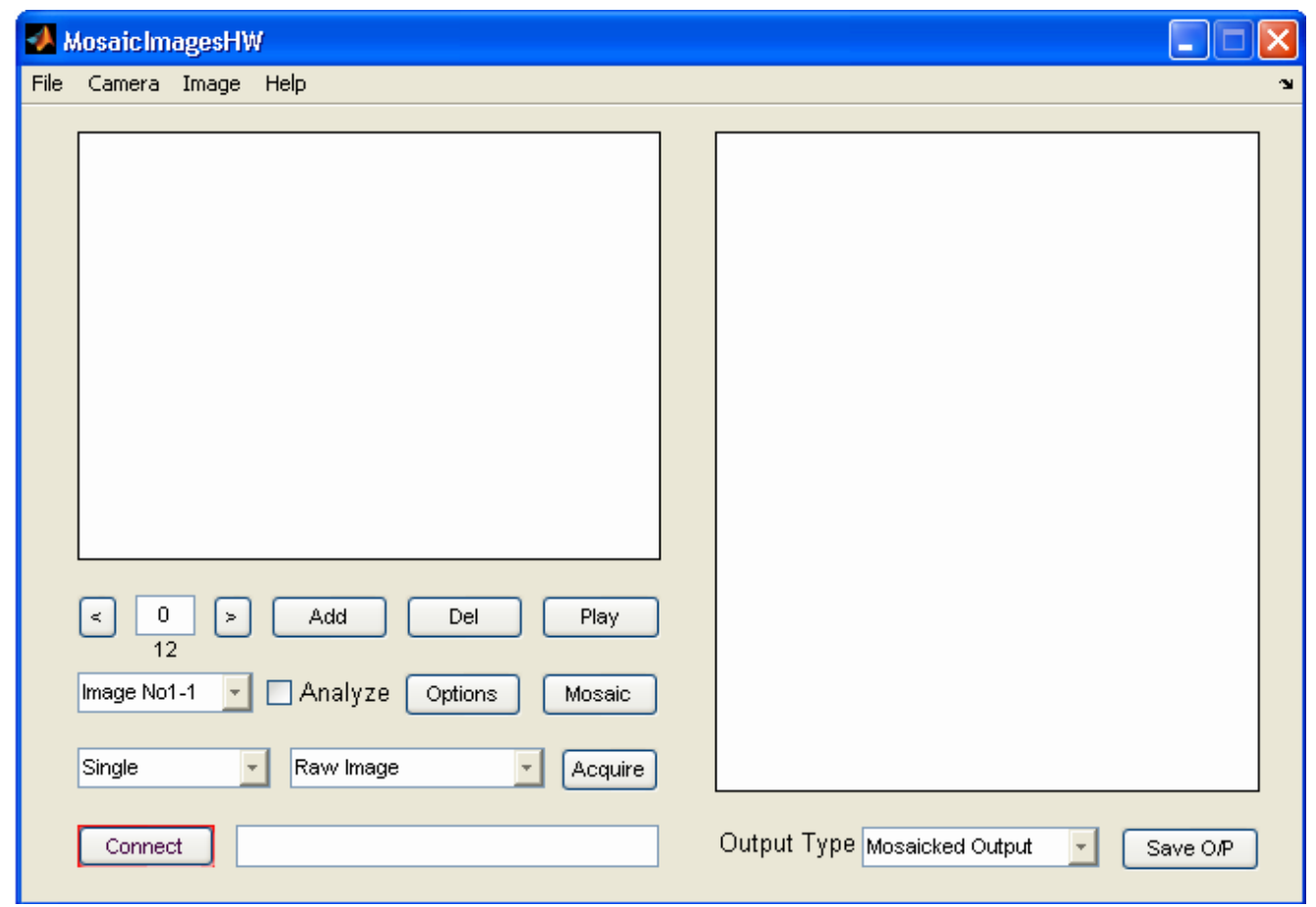

Figure A.10: Hardware assisted mosaicking GUI. 